\title{
Mapping of reentrant ventricular tachycardia in the rabbit heart
}

Citation for published version (APA):

Boersma, L. V. A. (1994). Mapping of reentrant ventricular tachycardia in the rabbit heart. [Doctoral Thesis, Maastricht University]. Datawyse / Universitaire Pers Maastricht.

https://doi.org/10.26481/dis.19940225lb

Document status and date:

Published: 01/01/1994

DOI:

10.26481/dis.19940225lb

Document Version:

Publisher's PDF, also known as Version of record

\section{Please check the document version of this publication:}

- A submitted manuscript is the version of the article upon submission and before peer-review. There can be important differences between the submitted version and the official published version of record.

People interested in the research are advised to contact the author for the final version of the publication, or visit the DOI to the publisher's website.

- The final author version and the galley proof are versions of the publication after peer review.

- The final published version features the final layout of the paper including the volume, issue and page numbers.

Link to publication

\footnotetext{
General rights rights.

- You may freely distribute the URL identifying the publication in the public portal. please follow below link for the End User Agreement:

www.umlib.nl/taverne-license

Take down policy

If you believe that this document breaches copyright please contact us at:

repository@maastrichtuniversity.nl

providing details and we will investigate your claim.
}

Copyright and moral rights for the publications made accessible in the public portal are retained by the authors and/or other copyright owners and it is a condition of accessing publications that users recognise and abide by the legal requirements associated with these

- Users may download and print one copy of any publication from the public portal for the purpose of private study or research.

- You may not further distribute the material or use it for any profit-making activity or commercial gain

If the publication is distributed under the terms of Article $25 \mathrm{fa}$ of the Dutch Copyright Act, indicated by the "Taverne" license above, 
MAPPING OF REENTRANT VENTRICULAR TACHYCARDIA IN THE RABBIT HEART 


\section{CIP-GEGEVEIVS KONINKLIJKE BIBLIOTHEEK DEN HAAG.}

Boersma, Lucas Victor August

Mapping of reentrant ventricular tachycardia in the rabbit heart / Lucas Victor August Boersma.- Madstricht.

Universitaire Pers Maastricht.- III.

Thesis Maastricht.- With ref.- With summary in Dutch.

ISBN 90-5278-127-3

Subject headings: ventricular tachycardia / antiarrhythmic drugs programmed electrical stimulation.

Lay-out: L.V.A. Boersma

Omslag: R. Leliveld

(C) 1994 L.V.A. Boersma, Maastricht 


\title{
MAPPING OF REENTRANT VENTRICULAR TACHYCARDIA IN THE RABBIT HEART
}

\author{
Proefschrift
}

\author{
ter verkrijging van de graad van doctor \\ aan de Rijksuniversiteit Limburg te Maastricht, \\ op gezag van de Rector Magnificus, Prof. dr. H. Philipsen, \\ volgens het besluit van het College van Dekanen, \\ in het openbaar te verdedigen op \\ vrijdag, 25 februari 1994 om 16.00 uur \\ door \\ Lucas Victor August Boersma
}

geboren op 2 februari 1965 te Heerlen 
Promotor: Prof.dr. M.A. Allessie

Co-promotor: dr. J. Brugada

Beoordelingscommissie:

Prof.dr. H.J.J. Wellens (voorzitter)

Prof.dr.ir. T. Arts

Prof.dr. M.J. Janse, Universiteit van Amsterdam

Prof.dr. H.J. Struijker Boudier

Prof.dr. A. Wit, Columbia University, New York

Het verschijnen van dit proefschrift werd mede mogelijk gemaakt door de financiële steun van de Nederlandse Hartstichting en de Stichting Wetenschappelijk Durrer-Fonds van het Interuniversitair Cardiologisch Instituut Nederland.

\section{ICIN}




\section{CONTENTS}

$\begin{array}{lr}\text { Chapter 1. General introduction } & 7\end{array}$

$\begin{array}{ll}\text { Chapter 2. The frozen heart model } & 25\end{array}$

Chapter 3. Functional reentry in anisotropic ventricular myocardium. Characteristics of the line of block during ventricular tachycardia

Chapter 4. Reentrant excitation around a fixed anatomical obstacle in uniform anisotropic ventricular myocardium

Chapter 5. The effects of $\mathrm{K}^{+}$on anisotropic conduction in sheets of perfused rabbit ventricular epicardium

Chapter 6. The effects of $\mathrm{K}^{+}$on reentrant ventricular tachycardia

Chapter 7. Mapping of reset of functional and anatomic reentry in anisotropic ventricular myocardium

Chapter 8. Entrainment of reentrant ventricular tachycardia in anisotropic rings of rabbit myocardium. Mechanisms of termination, changes in morphology, and acceleration.

Chapter 9. Effects of Heptanol, class Ic, and class III drugs on reentrant ventricular tachycardia. Importance of the excitable gap for the inducibility of double-wave reentry.

Chapter 10. Termination of anatomic ventricular tachycardia by class Ic and class III antiarrhythmic drugs.

Chapter 11. General discussion.

I. Summary

II. Samenvatting

III. Nawoord

IV. Publications

V. Curriculum Vitae 



\section{CHAPTER 1}

\section{INTRODUCTION}

1.1. Impulse propagation in ventricular myocardium

1.2. Reentry as a Mechanism for Cardiac Arrhythmias.

1.3. Ventricular arrhythmias after myocardial infarction.

1.4. Management of ventricular tachycardia in patients.

1.5. Aim of the study. 


\subsection{Impulse Propagation in the Heart}

The fact that electrical current is the basis for impulse propagation in the heart was first proposed by Engelmann in 1897. The technical development at that time did not allow a more detailed study of the nature of impulse propagation in the heart and for many years cardiac muscle was assumed to behave as a functional syncytium (Hodgkin and Huxley 1952, Hodgkin 1954). Sjöstrand and Andersson (1954) were among the first to propose that cardiac cells were individual units separated by intercalated discs. Subsequent studies revealed that specific low resistance structures in the intercalated discs, called nexuses or gap junctions (Veenstra and DeHaan 1988, Brink et al. 1988), were essential for the electrical current flow between adjacent myocardial fibers (Dewey and Barr 1962, Barr et al. 1965, De Mello 1982). Ventricular myocytes have a rectangular shape and mainly form end-to-end connections with adjacent cells (Sommer and Johnson 1979). Due to this specific (ultra)structure, the characteristics of impulse propagation in myocardium are influenced by the direction of propagation in relation to the cellular fiber orientation. Thus, cardiac tissue displays marked anisotropic conduction poperties (Lewis and Rothschild 1915, Dräper and Mya-Tu 1959, Sano et al. 1959).

\section{Anisotropic Conduction}

Dräper and Mya-Tu (1959) and Sano et al. (1959) were one of the first to accurately measure the differences in conduction velocity due to tissue anisotropy. They reported that conduction parallel to the fiber orientation was about 3 times faster than perpendicular to the fiber direction. Clerc (1976) showed that the lower conduction velocity in a transverse direction was correlated with a 9 times higher intercellular resistance compared to that in a longitudinal direction. The directional differences in resistance and conduction velocity result from the spatial distribution of the gap junctions. In a longitudinal direction, the myocardial fibers within a muscle bundle are well connected to each other through the intercalated discs (Sjöstrand and Anderson 1954). However, between parallel muscle bundles such gap junctions are scarce (Sommer and Johnson 1980) and the impulse has to travel a longer way to find a conducting pathway in a transverse direction. Per unit length an impulse must cross more gap junctions in a transverse than in a longitudinal direction. Since the gap junctional resistance is higher than the cytoplasmic resistance the transverse axial resistance $(625 \mathrm{Ohm} / \mathrm{cm}$, Roberts et al. 1979) will be higher than the longitudinal axial resistance (199 Ohm/cm, Roberts et al. 1979), resulting in slow conduction perpendicular to the myocardial fibers.

In a series of papers, Spach and co-workers (Spach et al. 1981, 1982a,b, 1984, 1986, 1987, 1988, 1989) adressed the implications of anisotropic impulse propagation for the occurrence of conduction disturbances. To characterize the conduction properties of the myocardium they used the parameter "safety factor for conduction" as introduced by Schmitt and Schmitt in 1940. The safety factor for conduction is defined as the ratio between the amount of generated excitatory current and the current required for successful impulse propagation. Spach et al. $(1981,1982)$ found that in a longitudinal direction the upstroke velocity of the action potential $\mathrm{dV} / \mathrm{dt} t_{\max }(\mathrm{Vmax})$ was lower, which was attributed to loss of excitatory current due to the low axial resistance parallel to the fiber orientation (Spach et al. 1981). In addition, they postulated that the 
membrane capacitance of the cells in longitudinal direction would be higher than perpendicular to the fiber orientation (Spach et al. 1982). As a result of these two factors the current supply would be low while the current demand would be high, resulting in a low safety factor for longitudinal conduction despite a high conduction velocity. For transverse conduction an opposite relation was found. Due to the high axial resistance the low conduction velocity was accompanied by a high Vmax and low membrane capacitance (Spach et al. 1981, 1982), resulting in a high safety factor for conduction. Thus, it was postulated that fast longitudinal conduction should be more vulnerable to conduction block than slow transverse conduction.

Apart from affecting conduction velocity, tissue anisotropy may also modify the spatial dispersion in refractoriness. Jalife and Moe (1981) observed that at high coupling resistances impulse propagation failed and the action potential distal to the area of high resistance was prolonged, while lowering the axial resistance had the opposite effect. In a computer model study, Joyner (1986) found that high coupling resistances enhanced the spatial dispersion in refractoriness. Abildskov et al. (1976) showed that the refractory period could also be modified by the direction of propagation. When an activation wave moved away from the site of measurement the refractory period was longer than when the activation wave propagated towards the site of measurement. Osaka et al. (1987) found that during transverse propagation the refractory period was longer than during longitudinal impulse propagation. In a recent study, Spach et al. (1989) made similar observations on the effects of anisotropy on the distribution and recovery of refractoriness.

\section{Effects of Modification of the Active and Passive Membrane Properties on Anisotropic Conduction}

The effects of a modification of the passive membrane properties on anisotropic conduction were studied by Delmar et al. (1987) and Balke et al. (1988) by applying heptanol. Apart from a small depression of the sodium conductance (Oxford and Swenson 1979) Heptanol mainly increases the intercellular resistance by blocking the gap junctions (Deleze and Herve 1983, Jalife et al. 1989). This preferentially slowed the conduction velocity transverse to the fiber orientation until conduction block occurred (Delmar et al. 1987, Balke et al. 1988). Spach et al. $(1982,1987)$ found that during rapid pacing transverse conduction was also preferentially depressed, which they attributed to an increase in gap junctional resistance by intracellular $\mathrm{Ca}^{++}$accumulation (Loewenstein 1966, De Mello et al. 1975, 1976, Weingart 1977).

In several studies the active membrane properties were depressed either by premature stimulation (Spach et al. 1981, 1982, 1988, van Capelle 1983), elevated extracellular K+ (Dominguez and Fozzard 1970, Tsuboi et al. 1985, Kléber et al. 1985, Delgado et al. 1990), or application of class I drugs (Kadish et al. 1986, Spach et al. 1982, 1987, 1988). The results of Spach et al. $(1981,1982,1988)$ concerning the effects of premature stimulation on anisotropic conduction where somewhat confusing. In early studies (Spach et al. 1981, 1982), during application of premature stimuli the conduction velocity was found to decrease proportionally to the decrease in Vmax. At increasing prematurity both longitudinal and transverse conduction velocity decreased with the same amount (Spach et al. 1982), despite the proposed lower safety factor for longitudinal impulse propagation. However, very early premature stimuli resulted in preferential longitudinal conduction block while transverse conduction remained intact, leading to reentrant excitation (Spach et al. 1981). In a later study, Spach et al. (1988) 
found that the effects of premature stimulation were different in uniform and nonuniform anisotropic human muscle preparations. In uniform anisotropic tissue, depression of the active membrane properties preferentially affected longitudinal conduction velocity but conduction block occurred at the same time both in a longitudinal and a transverse direction. In older non-uniform preparations, longitudinal impulse propagation was preferentially blocked or proceeded as zig-zag transverse conduction. The non-uniform anisotropic structure of older preparations was explained by formation of collagenous septa between adjacent muscle bundles which enhances the normal tissue anisotropy (Spach et al. 1986). Thus, uniform anisotropic media show more depression of longitudinal than transverse conduction but no directional preference for conduction block, while non-uniform anisotropic media show preferential longitudinal conduction block (Spach et al. 1988).

Studies on the effects of decreased active membrane properties by high concentrations of extracellular potassium have also showed contrasting results. Tsuboi et al. (1985) and Kléber et al. (1986) observed a preferential effect on longitudinal impulse propagation which is in agreement with the observations of Spach et al. (1981, 1982, 1988). However, Delgado et al. (1990) found that transverse conduction was preferentially depressed. Computer studies (Van Capelle 1983, Rudy et al. 1987) favour the results of Delgado et al. (1990) by showing that at low coupling resistances Vmax reached a stable value (Van Capelle 1983) while at high resistances Vmax decreased markedly (Rudy et al. 1987) finally resulting in transverse conduction block (Van Capelle 1983). Thus, despite the valid theoretical concept of directional differences in safety factor for conduction, a directional preference of depressed active membrane properties on anisotropic conduction remains disputed.

The effects of antiarrhyhmic drugs on anisotropic conduction have been tested in several studies (Spach et al. 1982, 1987, 1988, Kadish et al. 1986). The Na-K-ATPase inhibitor Ouabain preferentially depressed transverse conduction (Spach et al. 1982), presumedly due to an intracellular $\mathrm{Ca}^{++}$accumulation giving rise to an increase in intercellular resistance (Weingart 1977). The class I drugs quinidine and lidocaine preferentially decreased V $\max$ and conduction velocity in a longitudinal direction (Spach et al. 1987,1988). This would seem logical in view of the fact that by blocking the fast sodium channels and lowering Vmax, class I drugs would especially impair conduction in the direction with the lowest safety factor. In addition, in a longitudinal direction a greater use-dependent sodium channel binding of the drugs was found, since the depolarization phase lasted longer and the repolarization phase was shorter than in a transverse direction (Osaka et al. 1990, Spach et al. 1987). The observation of Kadish et al. (1986) that procainamide preferentially affects longitudinal conduction is in agreement with these results.

In summary, an increase in gap junctional resistance preferentially affects transverse impulse propagation while a depression of the active membrane properties seems to have a preferential effect on longitudinal conduction. However, especially for the latter postulation there is still a need for conclusive experimental evidence.

\subsection{Reentry as a Mechanism for Cardiac Arrhythmias}

Deviations from the normal sinus rhythm of the heart are in general attributed to abnormal impulse formation, abnormal impulse conduction, or a combination of both 
(Hoffman and Rosen 1981). Already a century ago the first investigations on the nature of deviations from the natural cardiac rhythm were performed. In 1897 McWilliam first postulated that apart from automaticity of muscle fibers, peristaltic movement of ventricular myocardium might result from continuous activation of the impulse over a number of connected muscle bundles. In later studies Mayer (1906, 1908), Mines (1913, 1914), and Garrey (1914, 1924) elaborated this concept which today is well known as reentrant excitation. They established that reentry could only be induced when after the occurrence of unidirectional conduction block, the cardiac impulse continued to propagate over an alternative pathway of slow conduction to reexcite the previous site of block from a different direction. As an ultimate test to prove that a an arrhythmia is based on reentry, the presumed reentrant circuit can be cut, to see whether the spontaneous activation cessates (Mines 1914).

One of the first preparations to fit these criteria was the classical model of Mines (1914) where reentry occurred around a fixed anatomical obstacle in rings of the tortoise ventricle. It was established (Mines 1914, Lewis 1925) that during reentry around a fixed anatomical obstacle the cycle length of the arrhythmia was determined by the length of the reentrant pathway and the conduction velocity. Because the cycle length exceeded the refractory period, in all segments of the circuit an excitable gap was present between the head and the tail of the circulating wave. In later studies it was found that reentrant activation could also occur without the involvement of a gross anatomical obstacle ("leading circle" reentry) (Allessie et al. 1976, 1977). As opposed to reentry around an anatomic barrier the cycle length of tachycardia was determined by the refractory period, implying that there is no excitable gap in the reentrant circuit. The size of these functionally determined circuits is determined by the wavelenght of the circulating impulse, defined as the product of conduction velocity and refractory period (Smeets et al. 1986, Rensma et al. 1988, Schalij et al. 1992).

Many studies have been performed to elucidate the possible mechanisms that favour the occurrence of reentry. Unidirectional block has been attributed to dispersion in refractoriness (Alessi et al. 1958, Han and Moe 1964, Allessie et al. 1976) while slow conduction was found to be due to local differences in excitability (Mines 1914, Schmitt and Erlanger 1928, Hoffman and Cranefield 1960). Dodge and Cranefield (1982) showed that geometrical factors such as asymmetrical non-uniformities in fiber diameter may contribute to slow conduction and conduction block. Only recently, Spach et al. (1981, 1982) provided evidence that tissue anisotropy could be a possible mechanism both for the occurrence of unidirectional conduction block and for slow impulse propagation. Tissue anisotropy and inhomogeneity in repolarization were found to be important determinants both for initiating and preventing atrial reentrant excitation (Spach et al. 1989).

In clinical practice, many different forms of reentrant excitation have been established such as atrial flutter around functional and/or anatomic obstacles (Waldo et al. 1977), circus movement tachycardia in the presence of accessory pathways (Durrer et al. 1967, Gallagher et al. 1978), bundle branch reentry (Moe 1965, Akhtar et al. 1978), and reentry in the Purkinje fiber network (Wit et al. 1972). In the course of myocardial infarction ventricular arrhythmias have been observed that are also most likely due to reentry (Wellens et al. 1972, 1978, Josephson et al. 1978a,b). 


\subsection{Ventricular Arrhythmias after Myocardial Infarction}

\section{Clinical Observations}

The occurrence of cardiac arrhythmias after myocardial infarction largely contributes to the mortality of coronary heart disease. In the acute phase of an ischemic event, cardiac arrest due to ventricular fibrillation is the main cause for the high mortality. Within the first year after myocardial infarction about 2-3\% of patients may experience episodes of spontaneous ventricular tachycardia (Mukharji et al. 1984, Richards et al. 1984) and in another 5-10\% of patients sudden death occurs probably due to ventricular tachycardia degenerating into ventricular fibrillation (Panidis et al. 1983, Kempf et al. 1984).

Wellens et al. were one of the first to provide clinical evidence for the mechanisms of ventricular tachycardia after myocardial infarction (VT). Durrer et al. (1967) and Wellens et al. $(1970,1971)$ demonstrated how premature stimuli could reproducibly initiate and terminate supraventricular tachycardia around an anatomical obstacle in patients with the Wolff-Parkinson-White syndrome. Wellens et al. (1972) applied the same principles to chronic ventricular tachycardia after myocardial infarction, showing that these arrhythmias are most likely based on reentrant activation. In the next years, Wellens et al. (1974, 1976, 1978) futher qualified and quantified the parameters that are involved in initiation and termination of reentrant ventricular tachycardia in patients.

These and other observations have provided evidence that ventricular tachycardia after myocardial infarction is due to reentrant activation: 1) the ease and reproducibility of both initiation and termination of VT by timed extrastimuli (Wellens et al. 1972, 1974, 1976, 1978, Buxton et al. 1984), 2) the presence of continuous electrical activity or middiastolic potentials during VT (Josephson et al. 1978, Horowitz et al. 1978), 3) the presence of complete reentrant circuits during intra-operative mapping of VT (Downar et al. 1988, de Bakker et al. 1988, 1990), 4) the possibility to reset VT by premature stimuli (Almendral et al. 1986a, Stamato et al. 1988), 5) fullfillment of the criteria for entrainment of VT (Waldo et al. 1982, 1989, Henthorn et al. 1988), and 6) abolition of VT by ablation of critical components of the reentrant circuit (Morady et al. 1991, 1993). However, much of our knowledge about the mechanisms of VT after myocardial infarction stems from experimental studies.

\section{Structural and Electrophysiological Substrate during Experimental Myocardial Infarction}

In the course of myocardial infarction the electrophysiologic behavior of the cells is completely changed and spontaneous ventricular arrhythmias occur. These events have been extensively and expertly reviewed by Wit and Janse (1989, 1992, 1993). The arrhythmias that occur after MI can be divided into an early ( $<30$ minutes), delayed (2472 hours) and later ( $>72$ hours) stage. Within the first 10 minutes of acute ischemia, the development of arrhythmias coincides with marked conduction slowing and fractionation of electrograms (Kaplinsky et al. 1979), due to depression of the active membrane properties (Janse et al. 1980, Kléber et al. 1990, Kléber et al. 1991). Pogwizd et al. (1987) used three-dimensional mapping to show that in this early phase the far majority of arrhythmias is based on reentrant activation (Janse et al. 1980, Janse 1990, Wit and Janse 1993). From 10 to 30 minutes after coronary occlusion the conduction abnormalities may dissappear (Kaplinsky et al. 1979), and the arrhythmias in this phase are thought to be associated with abnormal automaticity due to endogenous 
catecholamine release (Wilde et al. 1988, Wit and Janse 1993) although other mechanisms may be involved (Janse 1990). Harris (1950) facilitated the study of delayed arrhythmias (24-72 hours after occlusion) by developing the two stage coronary occlusion technique. In this subacute phase of ischemia, enhanced automaticity in surviving Purkinje fibers on the enodcardial surface has been shown to act as a source of tachycarrhythmias (Friedman et al. 1973, Basset 1990).

In the later stage of myocardial infarction, due to ischemia a part of the myocardium is destroyed, the size being determined by the specific coronary artery that is occluded and the level of the occlusion (Allen et al. 1950, Stephenson et al. 1960). A temporary occlusion followed by reperfusion of the infarcted area can preserve islands of mycocardium, interdigiting between necrotic tissue (Maroko et al. 1972, Karagueuzian et al. 1979, 1984, Michelson et al. 1980). In the 3-7 days after experimental occlusion of the left anterior descending artery, VT can usually be reproducibly induced and terminated by programmed electrical stimulation (El-Sherif et al. 1977, Karagueuzian et al. 1979, Michelson et al. 1980). The overall propensity for arrhythmias after complete coronary occlusion is slightly less compared to the arrhythmia inducibility of $50 \%$ in reperfused infarctions (Karagueuzian et al. 1979, Michelson et al. 1980). After complete occlusion of the left anterior descending coronary artery usually a transmural anteroseptal infarction results with thin layers of surviving muscle and Purkinje fibers overlying the infarcted area on the eodocardial and epicardial surface, designated as the "border zone" (El-Sherif et al. 1977, Karagueuzian et al. 1979, Michelson et al. 1980, Wit et al. 1982).

Both clinical and experimental studies have shown that the border zone of infarction exhibits electrophysiological and structural abnormalities, predisposing for reentrant arrhythmias (El-Sherif et al. 1977, Josephson et al. 1978, Karagueuzian et al. 1979, Michelson et al. 1980, Spear et al. 1983, 1990, Fenoglio et al. 1983, Richards et al. 1984, Ursell et al. 1985, Janse et al. 1989, Lesh et al. 1990, Wit et al. 1990, 1992). Michelson et al. (1980) found that in the first week after infarction the excitability thresholds and refractory periods were increased while Ursell et al. (1985) showed a reduction of the membrane resting potential and amplitude, upstroke velocity and duration of the action potential, returning to almost normal within two weeks after the occlusion. Studies by Boyden and coworkers (1990) on the ionic mechanisms for these changes showed that isolated cells from the epicardial border zone did show a normal membrane resting potential, but had a marked delay in recovery of Vmax. Furthermore, the transient outward current $\left(\mathrm{I}_{\mathrm{TOI}}\right)$ was decreased, leading to an alteration of the repolarization process (Boyden 1990).

In the early phase after infarction conduction velocities along the LAD are higher than perpendicular to the LAD, due to the uniform epicardial fiber orientation (Richards et al. 1984, Ursell et al. 1985). In the late phase, scar formation occurs and strands of newly formed fibrotic tissue separate the parallel muscle bundles (Karagueuzian et al. 1979, Michelson et al. 1980, Fenoglio et al. 1983, Ursell et al. 1985). Spear et al. (1990) applied heptanol to show an abnormal distribution of gap junctions and marginal electrical coupling in the infarcted area. This non-uniform anisotropic structure (Spach et al. 1987, 1988) of the infarcted area obscures the directional differences in impulse propagation (Ursell et al. 1985), and gives rise to fractionated electrograms with a long duration and very slow conduction (Richards et al. 1984, Ursell et al. 1985) which have also been observed in clinical infarctions (Josephson et al. 1978a,b, Horowitz et al. 1980). 


\section{Reentry as a mechanism for Ventricular Tachycardia}

Several studies have used high resolution mapping to provide direct evidence for reentrant activation in the border zone as the mechanism underlying ventricular tachycardia (El-Sherif et al. 1981, Wit et al. 1982, Mehra et al. 1983, Cardinal et al. 1988, Gough et al. 1985, Gallagher et al. 1985, Garan et al. 1987, Downar et al. 1988, De Bakker et al. 1988, 1990). In all these studies the reentrant circuits were accessible due to a location either on the epicardial or endocardial surface of the heart. In most experimental studies a model of 5 days old canine myocardial infarction has been used to investigate the nature of post-infarction VTs. Mapping of VT in this model has shown that the arrhythmia can be based on reentry around a single line of functional conduction block (Wit et al. 1982, Dillon et al. 1988) or on two circulating waves propagating in opposite direction around two separate lines of block with a cental common pathway (Figure-eight reentry) (El-Sherif et al. 1981, Mehra et al. 1983, Gough et al. 1985). Gough et al. (1985) proposed that spatial dispersion in refractoriness played an important role both in initiation and termination of ventricular tachycardia. Dillon et al. (1988) and Cardinal et al. (1988) showed that tissue anisotropy greatly modifies the characteristics of functionally determined reentrant circuits. Dillon et al. (1988) found that the central line of conduction block was usually oriented parallel to the fibers and sometimes consisted of pseudo-block when the impulse propagated very slowly transverse to the fibers. Schalij et al. (1988) also observed that in the majority of VTs in uniform anisotropic myocardium the central line of block was oriented parallel to the direction of the myocardial fibers. Due to the non-uniform anisotropic structure of the border zone, conduction in this area is very slow (Dillon et al. 1988, de Bakker et al. 1988). The resulting decrease in wavelength (Rensma et al. 1988, Schalij et al. 1988) allows reentry at a very small scale, further enhancing the chance on reentrant VT. The influence of the autonomic nervous system on initiation and termination of reentry in the border zone has been tested in several studies (Zuanetti et al. 1990, Butrous et al. 1992). Other than decreasing the action potential duration, isoproterenol hardly affected the active membrane properties of the cells (Zuanetti et al. 1990) while norepineprine reduced the spatial dispersion in refractoriness (Butrous et al. 1992). Both interventions improved conduction and reduced the occurrence of conduction block, thus preventing reentrant arhhythmias. On the other hand, Butrous et al. (1992) also showed that due to denervation hypersensitivity sympathetic stimulation increased the dispersion in refractoriness between the border zone and the normal myocardium, promoting the occurrence of reentry.

Although most experimental studies have shown that after myocardial infarction reentry occurs around a line of functional conduction block, the exact mechanism underlying ventricular tachycardia in patients remains to be determined. In clinical studies the existence of an endocardial border zone with slow conduction and fragmented electrograms has been shown (Josephson et al. 1978a,b, Fenoglio et al. 1983, de Bakke'r et al. 1988, 1990). Combined mapping and histological studies by de Bakker et al. $(1988,1990)$ have suggested that the endocardial origin of tachycardia (Horowitz et al. 1978) is an exit point of intramural macroreentrant pathways (Garan et al. 1987), although complete macroreentrant circuits have only been detected in a few hearts. However, for practical and technical reasons mapping of ventricular tachycardia in the human heart is only available in a subset of patients (de Bakker et al. 1988, 1990, Downar et al. 1988). Thus, there seems to be a need for indirect parameters to determine the mechanism underlying $V T$ in patients. 


\subsection{Management of Ventricular Tachycardia in Patients}

\section{Programmed electrical Stimulation}

The fact that programmed electrical stimulation has the propensity to initiate as well as terminate VT (Wellens et al. 1972, 1974, 1978, Karagueuzian et al. 1979, Michelson et al. 1980, El-Sherif et al. 1981, 1987, Buxton et al. 1984) makes it a very important tool in the management of this arrhythmia. From a diagnostic point of view programmed electrical stimulation has several virtues. The reproducibility and ease of inducibility of VT by premature stimulation indicate that reentry is the underlying mechanism (Wellens et al. 1972, 1978, Buxton 1984). In addition, Waldo et al. (1983, 1989) and Henthorn et al. (1988) developed four criteria for entrainment of VT, each of which can provide evidence for the reentrant nature of the arrhythmia. Okumura et al. (1985, 1987) have proposed that the criteria for entrainment could only be fullfilled when pacing was performed distal to the area of slow conduction. This was used to identify the relative position of the pacing site in the reentrant circuit and also to localize the position of the reentrant circuit in the ventricle (Almendral et al. 1988, Josephson et al. 1990). Several investigators have shown that single or double premature stimuli can reset ventricular tachycardia, again providing evidence for reentry as the responsible mechanism (Almendral et al. 1986a,b, Stamato et al. 1987, Rosenthal et al. 1988). Their results showed that during ventricular tachycardia a fully excitable gap existed, suggesting that reentry occurred around a central anatomic obstacle (Almendral et al. 1986a). The relative ease of termination of tachycardia (Almendral et al. 1986b) by a single premature stimulus suggested the existence of a weak link within the circuit. Several different pacing modalities have been used to identify such critical sites in the reentrant circuit for ablative therapy of ventricular tachycardia (Josephson et al. 1982, Marchlinski et al. 1987, Morady et al. 1991, Stevenson et al. 1992, Morady et al. 1993, Gürsoy et al. 1993).

In the therapy of ventricular tachycardia premature stimulation and entrainment both have proved to be of great value. Premature stimulation terminated tachycardia in 9 $36 \%$ of cases while entrainment was successful in about $49-60 \%$ of patients (Almendral et al. 1986b, Waldecker et al. 1986). Termination has been attributed to decremental propagation and block in the area of slow conduction (Okumura et al. 1987, Aizawa et al. 1992). Proarrhythmic effects like aggravation of existing tachycardias or induction of previously undocumented arrhythmias also have been reported in as much as $36 \%$ of patients (Waldecker et al. 1987).

So far, few studies have provided direct information by complete mapping of the events that occur during programmed electrical stimulation. El-Sherif et al. (1987), Waldecker et al. (1993) and Dillon et al. (1993) have shown that figure-eight reentry can be reset and entrained according to the criteria proposed by Waldo et al. $(1983,1987)$ and Henthorn et al. (1988). Termination of ventricular tachycardia was either due to conduction block in the central common pathway (El-Sherif et al. 1987, Waldecktr et al. 1993) or annihilation of the central lines of functional block (Waldecker et al. 1943). Acceleration of tachycardia occurred when during stimulation the lines of conduction block changed and a new circuit was created (El-Sherif et al. 1987, Dillon et al. 1993). Brugada et al. $(1990,1991)$ showed that acceleration of VT could be due to double-wave reentry, a change from one anatomical circuit to another, or a change from an anatomical to a functional circuit. 


\section{Antiarrhythmic Drugs}

Antiarrhythmic drug therapy for VT can have three major goals: 1) abolition of ventricular ectopy, 2) prevention of initiation, and/or 3) slowing or termination of ventricular tachycardia (Marchlinski 1990). As appropriate end points of therapy, a marked depression of inducibility, slowing in interval (>100 ms), and improved hemodynamic tolerance of ventricular tachycardia have been suggested (Marchlinski 1990). The class la drug procainamide, either alone or in combination with class Ib and Ic drugs or class III drugs has been shown to prevent induction of tachycardia in about $30 \%$ of patients (Marchlinski 1990). Class I drugs are frequently used because they can accomplish a use-dependent prolongation of the cycle length of ventricular tachycardia. Recent studies by Callans et al. (1991a), Markel et al. (1993), and Kidwell et al. (1993) showed that the rate of ventricular tachycardia was more effectively slowed by the class Ic drugs propafenone $(+48 \%)$ and flecainide $(+65 \%)$ than by the class Ia and Ib drugs procainamide $(+28-34 \%)$, quinidine $(+39 \%)$ and lidocaine $(+4 \%)$. In the study of Markel et al. (1993) it was shown that improved conduction during isoproterenol mardkely attenuated the drug-induced slowing of ventricular tachycardia during procainamide and quinidine, suggesting an effect of the autonomic system on the reentrant circuit. Results of Kay et al. (1987) suggested that antiarrhythmic drugs, in this case procainamide, preferentially depressed impulse propagation in the area of slow conduction, making this area vulnerable to conduction block and termination of ventricular tachycardia, either spontaneous or during premature stimulation.

As during programmed electrical stimulation, proarrhythmic effects have been observed during almost all antiarrhythmic drugs (Podrid 1987). For class Ia and class III drug the incidence of proarrhythmia is relatively low (1-8\%) and has been associated with development of torsade de pointes (Kadish et al. 1990, Woosley 1990). Class Ic drugs have a 9-15\% incidence of proarrhythmia (Podrid 1987, Horowitz et al. 1987) and have even been dispelled from the Cardiac Arrhythmia Suppression Trial (CAST Investigators 1989) because of a markedly increased mortality compared to other drugs.

Experimental evidence for the effects of drugs on ventricular tachycardia has so far been been scarce. In 5 days old canine infarctions El-Sherif et al. $(1977,1978)$ showed that lidocaine and diphenylhydantoin had a preferential effect on the reentrant pathway by increasing the refractory period and slowing conduction, thus decreasing the rate of tachycardia. Recent studies by Brugada et al. (1991) and Nassif et al. (1993) showed that low concentrations of Flecainide and Heptanol increased the cycle length of functionally determined VT by slowing conduction. The autonomic nervous system may attenuate the effects of drugs on VT by improving conduction and shortening refractoriness and its dispersion (Zuanetti et al. 1990, Butrous et al. 1992). However, more experimental studies are needed to understand the effects of anti-arrhythmic drugs on VT.

\subsection{Aim of the study.}

A vast number of clinical studies has provided a large amount of information on the effects of programmed electrical stimulation and antiarrhythmic drugs on the mechanisms underlying ventricular arrhythmias after myocardial infarction. A major limitation of almost all clinical studies is the lack of direct evidence by mappping of the events that occur during any of the studied interventions. For this reason experimental models of canine myocardial infarction have been developed, which are more easily 
accessible to mapping. These studies have greatly added to the understanding of the structural and electrophysiological changes that underly ventricular arrhythmias following myocardial infarction. However, so far the effects of programmed electrical stimulation and antiarrhythmic drugs have not been extensively tested in these models. A drawback of experimental infarction models is that the electrophysiological substrate is highly complex, making it more difficult to assess the contribution of individual electrophysiologic parameters on initiation, perpetuation, and termination of ventricular arrhythmias.

The studies presented in this thesis were performed for the following purposes:

a) development of an experimental substrate for reentrant ventricular tachycardia accessible to mapping.

b) assessment of the influence of dispersion in refractoriness and anisotropic tissue structure on the course of tachycardia.

c) determination of the effects of programmed electrical stimulation and antiarrhythmic drugs on ventricular tachycardia.

d) determination of the difference in characteristics of functional and anatomic reentrant ventricular tachycardia.

All of the studies described in this thesis were performed in a previously developed model of a two-dimensional layer of anisotropic ventricular subepicardium (Schalij et al. 1988, 1992), which is easily accessible to high-resolution mapping. The following questions were studied:

1) Is there a preferential directional effect of depressed active membrane properties on anisotropic conduction? This will be discussed in chapters 5 and 6 showing the effects of high extracellular $\mathrm{K}^{+}$during slow pacing in the intact epicardial layer and during VT around an anatomic obstacle.

2) What is the nature of the excitable gap during functionally determined anisotropic reentry? This question is adressed in chapters 3 and 7, showing the characteristics of functional VT and its response to high $\mathrm{K}^{+}$and premature stimuli.

3) What is the role of tissue anisotropy and dispersion of refractoriness for initiation and perpetuation of VT around a fixed anatomical obstacle? Chapter 4 will elaborate on the characteristics of macro-reentry in anisotropic ventricular myocardium.

4) Do functionally determined VT and anatomic VT have a characteristic response to premature stimulation and if so, what are the mechanisms involved? Chapter 7 will show the effects of premature stimuli during both types of VT.

5) How does anatomic VT respond to entrainment and what is the influence of a segment of slow transverse conduction on this response? This question is adressed in chapter 8 , showing the mechanisms by which entrainment terminates, changes the morphology, or accelerates VT.

6) What is the effect of various anti-arrhythmic drugs on anatomic VT and do they change the response to entrainment? Chapter 9 will show the effects on VT of three drugs with a different pharmacologic action, which modify the susceptibility to acceleration of VT.

7) Can anti-arrhythmic drugs terminate anatomic VT and what are the mechanisms involved? Chapter 10 will show two different mechanisms of termination of anatomic VT by class Ic and class III drugs. 


\section{REFERENCES}

Aizawa $Y$, Niwano S, Chinushi $M$, et al. Incidence and mechanism of interruption of reentrant ventricular tachycardia with rapid ventricular pacing. Circulation 1992;85:589-595

Akhtar M, Gilbert C, Wolf F, et al. Reentry within the His-Purkinje system. Elucidation of reentrant circuits using right bundle branch and His bundle recordings. Circulation 1978;58:295-304

Alessi R, Nusynowitz N, Abildskov J, et al. Nonuniform distribution of vagal effects on the atrial refractory period. Am J Physiol 1958;194:406-410

Allen J, Laadt J. The effect of the level of the ligature on mortality following ligation of the circumflex coronary artery in the dog. Am Heart J 1950;39:273-278

Allessie M, Bonke F, Schopman F. Circus movement in rabbit atrial muscle as a mechanism of tachycardia. II. The role of non-uniform recovery of excitability in the occurrence of unidirectional block, as studies with multiple microelectrodes. Circ Res 1976;39:9-18

Allessie M, Bonke F, Schopman F. Circus movement in rabbit atrial muscle as a mechanism of tachycardia. III. The "leading circle" concept: a new model of circus movement in cardiac tissue without the involvement of an anatomical obstacle. Circ Res 1977;41:9-18

Allessie M, Hoeks A, Schmitz G, et al. On-line mapping system for the visualization of the electrical activation of the heart. Int J Cardiac Imag 1986;2:59-63

Allessie M, Schalij M, Kirchhof $C$, et al. Experimental electrophysiology and arrhythmogenecity. Anisotropy and ventricular tachycardia. Eur Heart J 1989;10:E8-E12

Almendral J, Stamato N, Rosenthal M, et al. Resetting response patterns during sustained ventricular tachycardia: relationship to the excitable gap. Circulation 1986a;74:722-730

Almendral J, Rosenthal M, Stamato N, et al. Analysis of the resetting response in sustained uniform ventricular tachycardia: incidence and relation to termination. J Am Coll Cardiol 1986b;8:294-300

Almendral J, Gottlieb C, Rosenthal M, et al. Entrainment of ventricular tachycardia: explanation for surface electrocardiographic phenoma by analysis of electrograms recorded within the reentrant circuit. Circulation 1988;77:569-580

de Bakker J, Van Capelle F, Janse M, et al. Reentry as a cause of ventricular tachycardia in patients with chronic ischemic heart disease: electrophysiologic and anatomic correlation. Circulation $1988 ; 77: 589-606$

de Bakker J, Coronel R, Tasseron, et al. Ventricular tachycardia in the infarcted Langendorffperfused human heart: role of the arrangement of surviving cardiac fibers. J Am Coll Cardiol 1990;15:1594-1607

Balke C, Lesh M, Spear J, et al. Effects of cellular uncoupling on conduction in anisotropic canine ventricular myocardium. Circ Res 1988;63:879-892

Barr L, Dewey M, Berger W. Propagation of action potentials and the structure of the nexus in cardiac muscle. J Gen Physiol 1965;48:797-823

Basset A. Electrical activity in the 24 hour infarct. In Rosen M, Janse M, Wit A (Eds): Cardiac electrophysiology: a textbook. Futura Publishing Company, Mount Kisco, NY, 1990, pp. 755-772

Boyden P. Cellular electrophysiology of ischemic and infarcted tissues. In Rosen $M$, Janse $M$, Wit $A$ (Eds): Cardiac electrophysiology: a textbook. Futura Publishing Company, Mount Kisco, NY, 1990, pp. 673-694

Brink P, Mathias R, Jaslow S, et al. Steady-state current flow through gap junctions. Biophys J 1988;53:795-807

Brugada J, Boersma L, Kircchof C, et al. Double-wave reentry as a mechanism of ventricular tachycardia acceleration. Circulation 1990;81:1633-1643

Brugada L, Brugada $\mathrm{P}$, Boersma $\mathrm{L}$, et al. On the mechanisms for ventricular tachycardia acceleration during programmed electrical stimulation. Circulation 1991;83:1621-1629

Brugada J, Boersma L, Kirchhof C, et al. Proarrhythmic effects of Flecainide. Experimental evidence for increased susceptibility to reentrant arrhythmias. Circulation 1991;84:1808-1818

Buxton A, Waxman H, Marchlinski F, et al. Role of triple extrastimuli during electrophysiologic study of patients with documented sustained ventricular tachyarrhythmias. Circulation $1984 ; 69: 532-540$

Butrous G, Gough W, Restivo M, et al. Adrenergic effects on reentrant ventricular tachycardia in subacute myocardial infarction. Circulation 1992;86:247-254

Callans D, Marchlinski F. Characterization of spontaneous termination of sustained ventricular 
tachycardia associated with coronary artery disease. Am J Cardiol 1991;67:50-54

Callans D, Marchlinski F. Dissociation of termination and prevention of inducibility of sustained ventricular tachycardia with infusion of procainamide: evidence for distinct mechanisms. J Am Coll Cardiol 1992;19:111-117

Callans D, Hook B, Josephson M. The mechanism of propafenone-induced slowing of ventricular tachycardia in man as defined by analysis of resetting response patterns. PACE 1991;14:2035-2041

Cardinal R, Vermeulen M, Shenasa M, et al. Anisotropic conduction and functional dissociation of ischemic tissue during reentrant ventricular tachycardia in canine myocardial infarction. Circulation 1988;77:1162-1176

CAST-investigators. Preliminary report: effect of flecainide and encainidecin a randomized trial of arrhythmia suppression after myocardial infarction. New Engl J Med 1989;321:406-412

Clerc L. Directional differences of impulse spread in trabecular muscle from mammalian heart. J Physiol 1976;255:335-346

De Mello W. Effect of intracellular injection of calcium and strontium on cell communication in heart. J Physiol 1975;250:234-245

De Mello W. Influence of the sodium pump on intercellular communication in heart fibres: effect of intracellular injection of sodium ion on intercellular coupling. J Physiol 1976;263:171-179

De Mello W. Intercellular communications in cardiac muscle. Circ Res 1982;55:1-9

Deleze J, Herve J. Effect of several uncouplers of cell-to-cell communication on gap junction morphology in mammalian heart. J Membr Biol 1983;74:203-215

Delgado C, Steinhaus B, Delmar M, et al. Directional differences in excitability and margin of safety for propagation in sheep ventricular epicardial muscle. Circ Res 1990;67:97-110

Delmar M, Michaels D, Johnson T, et al. Effects of increasing intercellular resistance on transverse and longitudinal propagation in sheep epicardial muscle. Circ Res 1987;60:780-785

Dewey M, Barr L. A sudy of structure and distribution of the nexus. J Cell Biol 1964;23:553-585

Dillon S, Allessie M, Ursell P, et al. Influences of anisotropic tissue structure on reentrant circuits in the epicardial border zone of subacute canine infarcts. Circ Res 1988;63:182-206

Dillon S, Coromilas J, Waldecker B, et al. Effects of overdrive stimulation on functional reentrant circuits causing ventricular tachycardia in the canine heart. Mechanisms for resumption or alteration of tachycardia. J Cardiovasc Electrophysiol 1993 (in press)

Dodge F, Cranefield P. Nonuniform conduction in cardiac Purkinje fibers. In Carvalho A, Hoffman B, Lieberman $M$ (eds): Normal and abnormal conduction in the heart. Futura Publishing Co, Mount Kisco, NY, 1982, pp. 379-395

Dominguez G, Fozzard H. Influence of extracellular $\mathrm{K}+$ concentration on cable properties and excitability of sheep cardiac Purkinje fibers. Circ Res 1970;26:565-574

Downar E, Harris L, Mickleborough L, et al. Endocardial mapping of ventricular tachycardia in the intact human heart: evidence for reentrant mechanisms. J Am Coll Cardiol 1988;11:783-791

Dräper M, Mya-Tu M. A comparison of the conduction velocity in cardiac tissues of various mammals. Quart J Exptl Physiol 1959;44:91-109

Durrer D, Schoo L, Schuilenburg R, et al. The role of premature beats in the initiation and the termination of supraventricular tachycardia in the Wolff-Parkinson-White syndrome. Circulation 1967;36:644-662

El-Sherif N, Scherlag B, Scherlag R, et al. Reentrant arrhythmias in the late myocardial infarction period. 1. Conduction characteristics in the infarction zone. Circulation 1977;5:686-701

El-Sherif N. Reentrant ventricular arrhythmias in the late myocardial infarction period. 6 . Effect of the autonomic system. Circulation 1978;58:103-111

El-Sherif N, Smith A, Evans K. Canine ventricular arrhythmias in the late myocardial infarction period. 8. Epicardial mapping of reentrant circuits. Circ Res 1981;49:255-265

El-Sherif N, Gough W, Restivo M. Reentrant ventricular arrhythmias in the late myocardial infarction period. 14. Mechanisms of resetting, entrainment, acceleration, or termination of reentrant tachycardia by programmed electrical stimulation. PACE 1987;10:341-371

Engelmann Th. Ueber die leitung der Erregung im herzmuskel. Pflügers Arch Ges Physiol Mensch Tiene 1875;11:465-480

Fenoglio J, Pham T, Harken A, et al. Recurrent sustained ventricular tachycardia: structure and ultrastructure of subendocardial regions in which tachycardia originates. Circulation 1983;68:518533 
Friedman P, Stewart J, Wit A. Spontaneous and induced cardiac arrhythmias in subendocardial Purkinje fibers surviving extensive myocardial infarction in dogs. Circ Res 1973;33:612-626

Gallagher J, Pritchett E, Sealy W, et al. The pre-excitation syndromes. Prog Cardiovasc Dis 1978;20:285-327

Gallagher J, Del Rossi A, Fernandez J, Maranhao V, Strong M, White M, Gessman L. Cryothermal mapping of recurrent ventricular tachycardia in man. Circulation 1985;71:733-739

Garan H, Fallon J, Rosenthal S, et al. Endocardial, intramural, and epicardial activation patterns during sustained monomorphic ventricular tachycardia in late canine myocardial infarction. Circ Res 1987;60:879-896

Gardner P, Ursell P, Fenoglio J, et al. Electrophysiologic and anatomic basis for fractionated electrograms recorded from healed myocardial infarcts. Circulation 1985;72:596-611

Garrey $W$. The nature of fibrillatory contraction of the heart. Its relation to tissue mass and form. Am J Physiol 1914;33:397-414

Garrey W. Auricular fibrillation. Physiol Rev 1924;4:215-250

Gough W, Mehra R, Restivo M, et al. Reentrant ventricular arrhythmias in the late myocardial infarction period. 13. Correlation of activation and refractory maps. Circ Res 1985;57:432-442

Greenspan A, Spielman S, Webb C, et al. Efficacy of combination therapy with mexilitene and a type la agent for inducible ventricular tachyarrhythmias secondary to coronary artery disaease. Am J Cardiol 1985;56:277-284

Gürsoy S, Chiladakis I, Kuck K. First lessons from radiofrequency catheter ablation in patients with ventricular tachycardia. PACE 1993;16:687-691

Han J, Moe G. Nonuniform recovery of excitability in ventricular muscle. Circ Res 1964;14:44-60

Henthorn R, Okumura K, Olshansky B, et al. A fourth criterion for transient entrainment: the electrogram equivalent of progressive fusion. Circulation 1988;77:1003-1012

Hodgkin A. A note on conduction velocity. J Physiol 1954;125:221-224

Hodgkin A, Huxley A. A quantitative description of the membrane current and its application to conduction and excitation in nerve. J Physiol 1952;117:500-544

Hoffman B, Cranefield P. Electrophysiology of the heart. McGraw-Hill, New York, 1960

Hoffman B, Rosen M. Cellular mechanisms for cardiac arrhythmias. Circ Res 1981;49:1-15

Horowitz L, Josephson M, Harken A. Epicardial and endocardial activation during sustained ventricular tachycardia in man. Circulation 1980;61:1227-1238

Horowitz L, Greenspan A, Rae A, et al. Proarrhythmic responses during electrophysiologic testing. Am J Cardiol 1987;59:45E-48E

Jalife J, Moe G. Excitation, conduction, and reflection of impulses in isolated bovine and canine cardiac Purkinje fibers. Circ Res 1981;49:233-247

Jalife J, Sicouri S, Delmar M, Michaels D. Electrical uncoupling and impulse propagation in isolated sheep Purkinje fibers. Am J Physiol 1989;257:H179-H189

Janse M, van Capelle F, Morsink $\mathrm{H}$, et al. Flow of injury current and patterns of excitation during early ventricular arrhythmias in acute regional myocardial ischemia in isoloated porcine hearts. Evidence for two different arrhythmogenic mechanisms. Circ Res 1980;47:151-165

Janse M, Wit A. Electrophysiologic mechanisms of ventricular arrhythmias resulting from myocardial ischemia and infarction. Phys Reviews 1989;69:1049-1169

Janse $\mathbf{M}$. Electrical activity immediately following myocardial infarction. In Rosen M, Janse M, Wit A (Eds): Cardiac electrophysiology: a textbook. Fututra Publishing Company, Mount Kisco, NY, 1990, pp. 739-754

Janse $\mathbf{M}$, Kléber A. Propagation of electrical activity in ischemic and infarcted myocardium as the basis of ventricular arrhythmias. J Cardiovasc Electrophysiol 1992;3:77-87

Josephson M, Horowitz L, Farshidi A. Continuous local electrical activity. A mechanism of recurrent ventricular tachycardia. Circulation 1978;57:659-665

Josephson M, Horowitz L, Farshidi A, et al. Recurrent sustained ventricular tachycardia: 1 . Mechanisms. Circulation 1978;57:431-439

Josephson $\mathbf{M}$, Waxman $\mathrm{H}_{\text {, Cain }} \mathbf{M}$, et al. Ventricular activation during ventricular endocardial pacing. II. Role of pace-mapping to localize origon of ventricular tachycardia. Am J Cardiol 1982;50:11-22

Josephson ME. Treatment of ventricular arrhythmias after myocardial infarction. Circulation $1986 ; 74: 653-658$ 
Josephson M, Gottlieb C. Ventricular tachycardia associated with coronary artery disease. In: Cardiac Electrophsysiology. From cell to bedside. Eds. Zipes D, Jalife J, WB Saunders Company, 1990:571-580

Kadish A, Spear J, Levine J, et al. The effects of procainamide on conduction in anisotropic canine ventricular myocardium. Circulation 1986;74:616-625

Kadish A, Morady F. Torsade de Pointes. In: Cardiac Electrophysiology. From cell to bedside. Eds. Zipes D, Jalife J, WB Saunders Company, 1990:605-609

Kaplinsky E, Ogawa S, Balke C, Dreifus L. Two periods of early ventricular arrhythmia in the canine acute myocardial infarction model. Circulation 1979;60:397-403

Karagueuzian $\mathrm{H}$, Fenoglio J, Weiss $\mathrm{M}$, et al. Protracted ventricular tachycardia induced by premature stimulation of the canine heart after coronary artery occlusion and reperfusion. Circ Res 1979;44:833-846

Karagueuzian $\mathrm{H}$, Fenoglio J, Weiss $\mathrm{M}$, et al. Coronary occlusion and reperfusion: effects on subendocardial cardiac fibers. Am J Physiol 1980;238:H581-H593

Kay G, Epstein A, Plumb V. Preferential effect of procainamide on the reentrant circuit of ventricular tachycardia. J Am Coll Cardiol 1989;14:382-390

Kempf F, Josephson M. Cardiac arrest on ambulatory electrograms. Am J Cardiol 1984;53:1577-1582

Kidwell G, Greenspon A, Greenberg R, et al. Use-dependent prolongation of ventricular tachycardia cycle length by type I antiarrhythmic drugs in humans. Circulation 1993;87:118-125

Kléber A, Janse M, Wilms-Schopman F, et al. Changes in conduction velocity during acute ischeamia in ventricular myocardium of the isolated porcine heart. Circulation 1986;73:189-198

Kléber A, Janse M. Impulse propagation in myocardial ischemia. In: Cardiac Electrophysiology. From cell to bedside. Eds. Zipes D, Jalife J, WB Saunders Company, 1990:156-161

Kuchar D, Rottman J, Berger E, et al. The response to procainamide during electrophsyiologic study for sustained ventricular tachyarrhythmias predicts the response to other medication. J Am coll Cardiol 1988;12:982-988

Lesh M, Spear J, Moore N. Myocardial anisotropy: basic electrophysiology and role in cardiac rhythms. In: Cardiac Electrophysiology. From cell to bedside. Eds. Zipes D, Jalife J, WB Saunders Company, 1990:364-376

Lewis T, Rothschild M. The excitatory process in dog's heart. Part II. The ventricles. Philos Trans R Soc London 1915;206:181-226

Lewis T. Mechanism and graphic registration of the heartbeat. Chicago, IL. Chicago Medical, 1925:83-98

Littman L, Svenson R, Gallagher J, et al. Functional role of the epicardium in the post-infarction ventricular tachycardia: observations derived from computerized epicardial activation mapping, entraiment, and epicardial laser photoablation. Circulation 1991;83:1577-1591

Loewenstein W. Permeability of membrane junctions. Ann NY Acad Sci 1966;137:441-472

Marchlinski F, Almendral J, Cassidy D, et al. Localization of endocardial site for catheter ablation of ventricular arrhythmia. In: Ablation in cardiac arrhythmias. Eds. Fontaine G, Scheinman, Futura Publishing Company, Mount Kisco NY, 1987:289-309

Marchlinski F. Ventricular tachycardia: clinical presentation, course, and therapy. In: Cardiac Electrophysiology. From cell to bedside. Eds. Zipes D, Jalife J, WB Saunders Company, 1990:756777

Markel M, Miles W, Luck J, et al. Differential effects of isoproterenol on sustained ventricular tachycardia before and during procainamide and quinidine antiarthythmic therapy. Circulation 1993;87:783-792

Maroko P, Libby P, Ginks W, et al. Coronary artery reperfusion. 1. Early effects on local myocardial function and the extent of myocardial necrosis. J Clin Invest 1972:51:2710-2716

Mayer A. Rhythmical pulsation in scyphomedusae. Carnegie Institute, Publ 47:1, 1906

Mayer A. Rhythmical pulsation in scyphomedusae II. Papers from the tortugas laboratory of the Carnegie Institute, Publ 102, 1908

McWilliam J. Fibrillar contraction of the heart. J Physiol 1897;8:296-310

Mehra R, Zeiler R, Gough W, et al. Reentrant ventricular arrhythmias in the late myocardial infarction period. 9. Electrophysiologic anatomic correlation of reentrant circuits. Circulation 1983;67:11-24

Michelson E, Spear J, Moore E. Electrophysiologic and anatomic correlates of sustained ventricular 
tachyarrhythmias in a model of chronic myocardial infarction. Am J Cardiol 1980;45:583-590

Mines G. On dynamic equilibrium in the heart. J Physiol 1913;46:349-383

Mines G. On circulating excitations in heart muscles and their possible relation to tachycardia and fibrillation. Trans R Soc Canada 1914;sect 5:43-53

Morady F, Kadish A, Rosenheck S, et al. Concealed entrainment as a guide for catheter ablation of ventricular tachycardia in patients with prior myocardial infarction. J Am Coll Cardiol 1991;17:678-689

Morady F, Harvey M, Kalbfleisch S, et al. Radiofrequency catheter ablation of ventricular tachycardia in patients with coronary artery disease. Circulation 1993;87:363-372

Mukharji J, Rude R, Pode W, et al. Risk factors for sudden death after acute myocardial infarction: two year follow-up. Am J Cardiol 1984;54:31-36

Nassif G, Dillon S, Rayhill S, Wit A. Reentrant circuits and the effects of heptanol in a rabbit model of infarction with a uniform epicardial border zone. J Cardiovasc Electrophysiol 1993;4:112-133

Osaka T, Kodama I, Tsuboi N, et al. Effects of activation sequence and anisotropic cellular geometry on the repolarization phase of action potential of dog ventricular muscles. Circulation 1987;76:226236

Okumura K, Henthorn R, Epstein A, et al. Further observations on transient entrainment: importance of pacing site and properties of the components of the reentrant circuit. Circulation 1985;72:1293-1307

Okumura K, Olshansky B, Henthorn R, et al. Demonstration of the presence of slow conduction during sustained ventricular tachycardia in man: use of transient entrainment. Circulation 1987;75:369-378

Oxford G, Swenson R. n-Alkanols potentiate sodium channel inactivation in squid giant axons. Biophys J 1979;26:585-590

Panidis I, Morganroth J. Sudden death in hospitalized patients: cardiac rhythm disturbances detected by ambulatory electrocardiographic monitoring. J Am Coll Cardiol 1983;2:798-805

Pogwizd S, Corr P. Reentrant and non-reentrant mechanisms contribute to arrhythmogenesis during early myocardial ischemia: results using three-dimensional mapping. Circ Res 1987;61:352-371

Pogwizd S, Corr P. Electrophysiologic mechanisms underlying arrhythmias due to reperfusion of ischemic myocardium. Circulation 1987;76:404-426

Podrid P, Lampert S, Graboys T, et al. Aggravation of arrhythmia by anti-arrhythmic drugs: Incidence and predictors. Am J Cardiol 1987:59:38E-44E

Rensma P, Allessie M, Lammers $W$, et al. The length of the excitation wave as an index for the susceptibility to reentrant atrial arrhythmias. Circ Res 1988;62:395-402

Richards D, Cody D, Deniss A, et al. Ventricular electrical instability: a predictor of death after myocardial infarction. Am J Cardiol 1983;51:75-80

Richards D, Blake G, Spear J, et al. Electrophysiologic substrate for ventricular tachycardia: correlation of properties in vivo and in vitro. Circulation 1984;69:369-381

Roberts D, Hersh L, Scher A. Influence of cardiac fiber orientation on wavefront voltage, conduction velocity, and tissue resistivity. Circ Res 1979;44:701-712

Rosenthal M, Stamato N, Almendral J, et al. Resetting of ventricular tachycardia with electrocardiographic fusion: incidence and significance. Circulation 1988;77:581-588

Rudy Y, Quam W. Model study of the effects of the discrete cellular structure on electrical impulse propagation. Circ Res 1987;61:815-823

Sano T, Takayama N, Shimamoto T. Directional difference of conduction velocity in the cardiac ventricular syncytium studied by micorelectrodes. Circ Res 1959;7:262-268

Schalij M. Anisotropy and ventricular tachycardia. Thesis, University of Limburg, Maastricht, The Netherlands, 1988

Schalij M, Lammers W, Rensma P, et al. Arisotropic conduction and reentry in perfused epicardium of rabbit ventricle. Am J Physiol 1992;263:H1466-H1478

Schmitt F, Erlanger J. Directional differences in the conduction of the impulse through heart muscle and their possible relation to extrasystolic and fibrillary contractions. Am J Physiol 1928;87:326347

Schmitt F, Schmitt O. Partial excitation and variable conduction in the squid giant axon. J Physiol 1940;98:26-46

Sjöstrand F, Anderson E. Electron microscopy of the intercalated discs of cardiac muscle tissue. 
Experientia 1954;10:369-372

Smeets J, Allessie $M$, Lammers $W$, et al. The wavelength of the cardiac impulse and reentrant arrhythmias in isolated rabbit atrium. The role of heart rate, autonomic transmitters, temperature, and potassium. Circ Res 1986;58:96-108

Sommer J, Johnson E. Ultrastructure of cardiac muscle. In: Handbook of Physiology sect. II, vol. I, The Heart. Eds. Berne R, Sperelakis N, Geiger S. Bethesda, American Physiological Society, 1980:130-186

Spach M, Miller W, Geselowitz D, et al. The discontinuous nature of propagation in normal canine cardiac muscle. Evidence for recurrent discontinuities of intracellular resistance that affect the membrane currents. Circ Res 1981;48:35-54

Spach M, Miller W, Dolber $P$, et al. The functional role of structural complexities in the propagating depolarization in the atrium of the dog. Cardiac conduction disturbances due to discontinuities of effective axial resistivity. Circ Res 1982;50:175-191

Spach M, Kootsey J, Sloan J. Active modulation of electrical coupling between cardiac cells of the dog. A mechanism for transient and steady-state variations in conduction velocity. Circ Res 1982;51:347-362

Spach M, Dolber P. Relating extracellular potentials and their derivatives to anisotropic propagation at a microscopic level in human cardiac muscle. Evidence for electrical uncoupling of side-to-side fiber connections with increasing age. Circ Res 1986;58:356-371

Spach M, Dolber P, Heidlage J, et al. Propagating depolarization in anisotropic human and canine cardiac muscle: apparent directional differences in membrane capacitance. A simplified model for selective directional effects of modifying the sodium conductance on Vmax, Tfoot, and the propagation safety factor. Circ Res 1987;60:206-219

Spach M, Dolber P, Heidlage J. Influence of the passive anisotropic properties on directional differences in propagation following modification of the sodium current in human atrial muscle. A model of reentry based on anisotropic discontinuous propagation. Circ Res 1988;62:811-832

Spach M, Dolber P, Heidlage F. Interaction of inhomogeneities of repolarization with anisotropic propagation in dog atria. A mechanism for both preventing and initiating reentry. Circ Res $1989 ; 65: 1612-1631$

Spear J, Mickelson E, Moore N. Cellular electrophysiologic characteristics of chronic infarcted myocardium in dogs susceptible to sustained ventricular tachycardia. I Am Coll Cardiol 1983;1099-1110

Spear J, Balke C, Lesh M, et al. Effect of cellular uncoupling by heptanol on conduction in infarcted myocardium. Circ Res 1990;66:202-217

Stamato N, Rosenthal M, Almendral J, Josephson $\mathrm{M}$. The resetting response of ventricular tachycardia to single and double extrastimuli: implications for the excitable gap. Am J Cardiol 1987;60:596-601

Stephenson S, Cole R, Parrish T, et al. Ventricular fibrilation during and after coronary artery occlusion: incidence and protection afforded by several drugs. Am J Cardiol 1960;5:77-87

Stevenson W, Hassan $H$, Sager $P$, et al. Targeting endocardial sites for catheter ablation of ventricular tachycardia after myocardial infarction. Circulation (abstr) 1992;86:I-519

Tsuboi N, Kodama I, Toyama J, et al. Anisotropic conduction properties of canine ventricular muscle. Influences of high extracellular $\mathrm{K}+$ concentration and stimulation frequency. Jpn Circ J 1985;49:487-498

Ursell P, Gardner P, Albela A, et al. Structural and electrophysiological changes in the epicardial border zone of canine myocardial infarcts during infarct healing. Circ Res 1985;56:436-451

Van Capelle F. Slow conduction and cardiac arrhythmias. Thesis, University of Amsterdam, Amsterdam, The Netherlands, 1983

Vaughan Williams E. A classification of antiarrhythmic actions reassessed after a decade of new drugs. J Clin Pharmacol 1984;24:129-143

Veenstra R, DeHaan R. Measurement of single channel currents from cardiac gap junctions. Science 1986;233:972-974

Waldecker B, Brugada P, Zehender M, et al. Modes of electrical termination of ventricular tachycardia: importance for the selection of implantable antitachycardia device. Am J Cardiol 1986;57:150-155

Waldecker B, Coromilas J, Saltman A, et al. Overdrive stimulation of functional reentrant circuits 
causing ventricular tachycardia in the infarcted canine heart. Resetting and entrainment. Circulation 1993;88:

Waldo A, MacLean W, Karp R, et al. Entrainment and interruption of atrial flutter with atrial pacing: studies in man following open-heart surgery. Circulation 1977;56:737-748

Waldo A, Plumb V, Arciniegas J, et al. Transient entrainment and interruption of the atrioventricular bypass pathway type of paroxysmal atrial tachycardia. Circulation 1983;67:73-83

Waldo A, Henthorn R. Use of transient entrainment during ventricular tachycardia to localize a critical area in the reentry circuit for ablation. PACE 1989;12:231-244

Weingart $\mathbf{R}$. The actions of ouabain on intercellular coupling and conduction velocity in mammalian ventricular muscle. J Physiol 1977;264:341-365

Wellens H, Schuilenburg R, Durrer D. Electrical stimulation of the heart in patients with WolffParkinson-White syndrome, type A. Circulation 1970;42:271-

Wellens HJJ. Electrical stimulation of the heart in the study and treatment of tachycardia. Doctoral Thesis, University of Leiden, the Netherlands. Stenfert Kroese, Leiden 1971

Wellens H, Schuilenburg R, Durrer D. Electrical stimulation of the heart in patients with ventricular tachycardia. Circulation 1972;46:216-226

Wellens $\mathrm{H}$, Lie Kl, Durrer D. Further observations on ventricular tachycardia as studied by electrical stimulation of the heart. Chronic recurrent ventricular tachycardia and ventricular tachycardia during acute myocardial infarction. Circulation 1974;49:647-653

Wellens H, Durrer D, Lie Kl. Observations on the mechanisms of ventricular tachy-cardia in man. Circulation 1976;54:237-244

Wellens H. Value and limitations of programmed electrical stimulation of the heart in the study and treament of tachycardias. Circulation 1978;57:845-853

Wilde A, Peters R, Janse M. Catecholamine release and potassium accumulation in the isolated globally ischemic rabbit heart. J Mol Cell Cardiol 1988;20:887-896

Wit A, Cranefield P, Hoffman B. Slow conduction and reentry in the ventricular conducting system. II. Single and sustained circus movement in networks of canine and bovine Purkinje fibers. Circ Res 1972;30:11-22

Wit A, Allessie M, Bonke F, et al. Electrophysiologic mapping to determine the mechanism of experimental ventricular tachycardia initiated by premature impulses. Experimental approach and initial results demonstrating reentrant excitation. Am J Cardiol 1982;49:166-185

Wit A, Dillon S. Anisotropic reentry. In: Cardiac Electrophysiology. From cell to bedside. Eds. Zipes D, Jalife J, WB Saunders Company, 1990:353-363

Wit A, Janse M. Experimental models of ventricular tachycardia and fibrillation caused by ischemia and infarction. Circulation 1992;85 (suppl I):131-I42

Wit A, Janse $\mathrm{M}$. The ventricular arrhythmias of ischemia and infarction. Electrophysiologic mechanisms. Futura Publishing Company, Mount kisco, NY, 1993

Woosley R. Antiarrhythmic agents: pharmacokinetics and pharmacodynamics. In: Cardiac Electrophysiology. From cell to bedside. Eds. Zipes D, Jalife J, WP Saunders Company, 1990:872882

Zuanetti R, Hoyt R, Corr P. $\beta$-adrenergic mediated influences on microscopic conduction in epicardial regions overlying infarcted myocardium. Circ Res 1990;67:284-302 
CHAPTER 2

THE FROZEN HEART MODEL 


\section{Introduction}

Due to the ultrastructure of cardiac muscle $e^{1}$ the intercellular resistance parallel to the fiber orientation is 10 times lower than in a transverse direction, ${ }^{2}$ allowing an about 3 times higher conduction velocity parallel to the fiber axis., ${ }^{3,4}$ Spach et al. ${ }^{4 y}$ proposed that despite the higher conduction velocity, a lower axial resistance together with a higher effective membrane capacitance would result in a lower safety factor for longitudinal impulse propagation. In several studies they showed that when the active membrane properties were depressed, longitudinal conduction block occurred first before slow conduction transverse to the fiber orientation was blocked, fulfilling all the prerequisites for intramural reentry. ${ }^{5-9}$ Under normal circumstances the anisotropic conduction properties of ventricular myocardium are not apparent because of the three-dimensional structure of the tissue. The wall of the left ventricle consists of several layers of myocardium in which the rod-shaped muscle fibers are oriented parallel to each other. ${ }^{1}$ The fiber direction in these layers changes over an angle of about 120 degrees from the endocardial to the epicardial surface of the ventricle, ${ }^{10}$ resulting in equally fast conduction in every direction. To study the influence of directional differences in conduction properties on ventricular conduction disturbances and induction of reentrant arrhythmias, the "frozen heart" model was developed by Schalij et al. ${ }^{11,12}$ A thin layer of anisotropic Langendorff-perfused subepicardium was created by cryodestruction of the endocardial and intramural layers of the rabbit left ventricle. This chapter will summarize the histologic and electrophysiologic characterization of this model, as previously published in more detail elsewhere. ${ }^{11-14}$ Since then, other investigators ${ }^{15,16}$ have adopted this model and reported similar morphological and electrophysiological characteristics.

\section{Preparation}

"Flamish (iiant" rabbits weighing between 3.5-4.5 kg were used. All animal handling and operation procedures were performed according to the guidelines of the American Physiological Society and approved by the Animal Investigations Com-mittee of the University of Limburg. After heparinization (1000 U.I.) and anesthesia with $0.5 \mathrm{ml} / \mathrm{kg}$ of Hypnorm i.m. (10 mg fluanison and $0.2 \mathrm{mg}$ phentanyl per $\mathrm{ml}$ ) the rabbits were killed by a cervical dislocation. The heart was rapidly excised through a midsternal thoracotomy and placed in cold Tyrode's solution at $10^{\circ} \mathrm{C}$. Subsequently, the aorta was cannulated and connected to a Langendorff perfusion system. The perfusion pressure was kept constant at $60 \mathrm{~mm} \mathrm{Hg}$ resulting in a flow of $65 \pm 8 \mathrm{ml} / \mathrm{min}(\mathrm{n}=10)$. The heart was perfused with Tyrode's solution with the following millimolar composition: $\mathrm{NaHCO}_{3} 20.1, \mathrm{NaH}_{2} \mathrm{PO}_{4} 1.2, \mathrm{NaCl} 130, \mathrm{KCl} 5.6, \mathrm{CaCl}_{2} 2.2, \mathrm{MgCl}_{2}$ 0.6, and glucose 12. The solution was saturated with carbogen $\left(95 \% \mathrm{O}_{2}\right.$ and $\left.5 \% \mathrm{CO}_{2}\right), \mathrm{pH}$ was 7.35 and the temperature was $37^{\circ} \mathrm{C}$.

To create a thin two-dimensional layer of left ventricular muscle a cryprocedure was performed. First, a plastic cannula was inserted in the right ventricular cavity and filled with liquid nitrogen $\left(-196^{\circ} \mathrm{C}\right)$ until the complete right ventricle was cryo-ablated. This resulted in a reduction of the coronary flow to $53 \pm 10 \mathrm{ml} / \mathrm{min}(\mathrm{n}=10)$. The cannula was then inserted into the left ventricular cavity through an incision in the left atrium (Figure 2.1). The heart was then immersed in a tissue bath containing Tyrode's solution at $30^{\circ} \mathrm{C}$. Then, the coronary flow was temporarily interrupted and the cannula was filled with liquid nitrogen for 7 minutes. During endocardial freezing of the left ventricle the temperature in the tissue bath was maintained between 29.5 and $30.5^{\circ} \mathrm{C}$ 


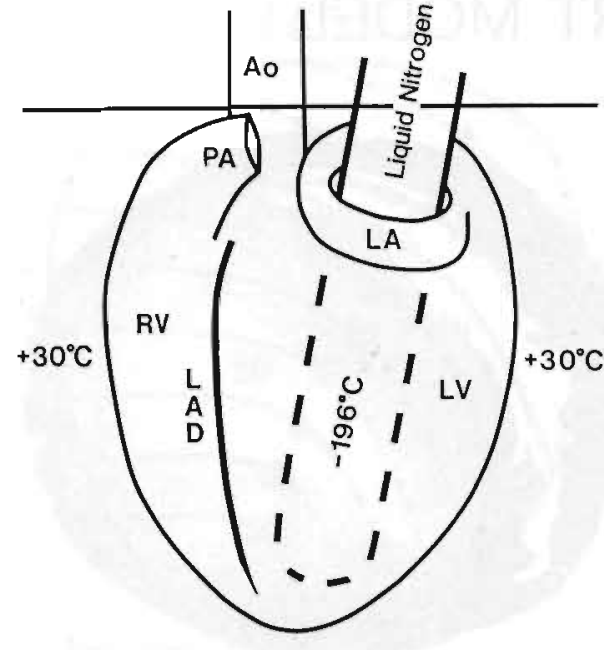

Tìssue Bath
Figure 2.1: Schematic drawing of the cryoprocedure to obtain thin layers of anisotropic left ventricular subepicardium. The heart was cannulated and perfused through the aorta. A hollow plastic cannula was inserted in the left ventricular cavity through an incision in the left atrium. The cannula was then filled with liquid nitrogen $\left(-196^{\circ} \mathrm{C}\right)$ for 7 minutes. During this procedure the coronary flow was inter-rupted and the heart was immersed in a tissue bath containing Tyrode's solution at $+30^{\circ} \mathrm{C}$. $A 0=$ Aorta, $P A=$ Pulmonary Artery, $R V=$ Right Ventricle, $L A D=$ Left Anterior Descending artery, $L V=$ Left Ventricle, $L A=$ Left Atrium.

to prevent cryodestruction of the subepicardial layers. After 7 minutes the coronary flow was restored and the heart was kept in the warm tyrode for another 3 minutes before it was lifted from the tissue bath. After the cryo-procedure the coronary flow had decreased to $27 \pm 11 \mathrm{ml} / \mathrm{min}(\mathrm{n}=10)$.

\section{Morphological Characteristics}

After the experiments the heart was stained either with buffered 2,3,5-triphenyltetrazolium-chloride ${ }^{18}$ or nitroblue tetrazolium ${ }^{19}$ to study the histological features of the preparation. Both substances are specific markers for dehydro-genase activity, exposing surviving muscle fibers by transforming into a bright red or blue colour respectively. This allowed a macroscopic determination of the thickness of the surviving tissue layer. The hearts were cut in 2-mm thick sections parallel to the atrioventricular ring from base to apex. Examination of these slices showed that the complete intraventricular septum, and about $80 \%$ of the intramural layers at the endocardial side of the left ventricle were completely destroyed (Figure 2.2). Only a thin layer of subepicardium survived, with an average thickness of $1.0 \pm 4 \mathrm{~mm}(\mathrm{n}=99)$ in the studies of Schalij et al..$^{12,13}$ and $1.1 \pm 0.3 \mathrm{~mm}(\mathrm{n}=10) \mathrm{mm}$ found by Brugada et al. ${ }^{15}$. There was a sharp and sudden transition between surviving and dead tissue. No islands of viable tissue were found in the frozen parts of the heart while in the surviving layer no islands of dead tissue were observed. ${ }^{12,13,15}$ However, at some sites the presence of epicardial fat or blood vessels diminished the thickness of the surviving myocardium. Previously, it has been shown that in the intact left ventricle, the fiber orientation in different intramyocardial layers varies over about 120 degrees from the endocardial to the epicardial surface of the heart, while at the apex of the heart these different layers merge into a complex vortex-like structure. ${ }^{11}$

In the surviving epicardial layer of the rabbit left ventricle the epicardial fibers were orientated in a pattern as shown in figure 2.3. Close to the Left Anterior Descending coronary artery the fibers were oriented almost parallel to the atrio-ventricular ring. At the center and towards the posterior side of the left ventricular free wall, the fiber axis 


\section{FROZEN HEART MODEL}
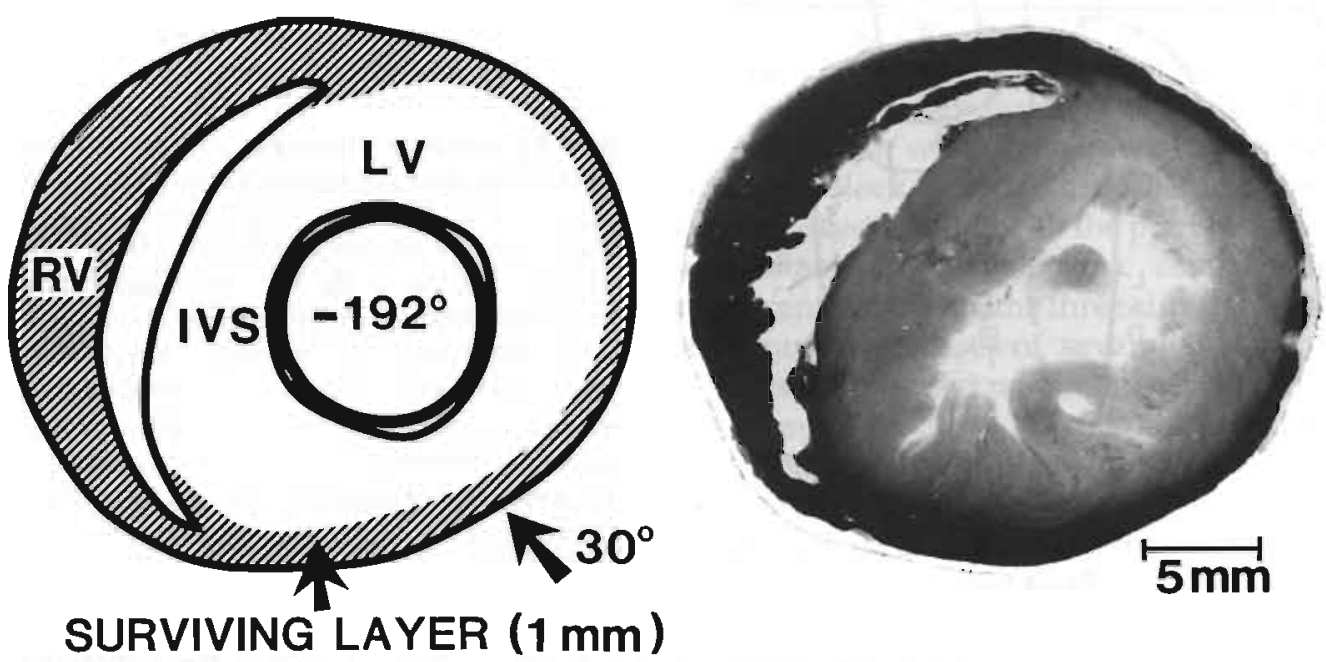

SURVIVING LAYER $(1 \mathrm{~mm})$

Figure 2.2: Creation of a thin subepicardial layer by endocardial freezing. Left: By filling the cannula with liquid nitrogen $\left(-196^{\circ} \mathrm{C}\right)$ for 7 minutes, the interventricular septum and the endocardial and $80 \%$ of the intramural layers of the left ventricle are destroyed (white area). The warm $\left(+30^{\circ} \mathrm{C}\right)$ tyrode's solution in the tissue bath protects the outside of the left ventricle subepicardium, resulting in a thin (1 $\mathrm{mm})$ surviving layer of subepicardium (dashed area). $R V=$ right ventricle, IVS= interventricular septum, $L V=$ left ventricle. Right: Example of a slice of the heart stained by 2,3,5-triphenyl-tetrazolium-chloride. A dark staining exposes the surviving ventricular muscle.

gradually curved towards the apex with an angle of up to 80 degrees relative to the atrioventricular ring. Thus, the surviving epicardial layer showed a clear anisotropic tissue structure. Chapter 4 of this thesis will provide more detailed data on the fiber orientation in different segments of the free wall of the left ventricle.

\section{Electrophysiological characteristics of the epicardial layer}

Schalij et al. ${ }^{12,13}$ determined the refractory periods before and after the cryo-procedure at 9 sites in the left ventricular free wall. During pacing at $350 \mathrm{~ms}$ the refractory periods were not changed by the cryoprocedure (ANOVA, n.s.). Figure 2.4 shows that the spatial dispersion in refractoriness was very small, the ERP ranging from $167 \pm 10$ to 175 $\pm 7 \mathrm{~ms}$ before freezing and from $165 \pm 9$ to $175 \pm 7$ after freezing. There was no anatomically defined area with a significantly different refractory period than at other sites (Bonferroni's t-test, n.s.). Schalij et al. ${ }^{12,13}$ showed that the cryoprocedure did not significantly affect the longitudinal $\left(\theta_{\mathrm{L}}\right)$ and transverse $\left(\theta_{\mathrm{T}}\right)$ conduction velocities at the epicardial surface. During pacing at $350 \mathrm{~ms}$ intervals $\theta_{\mathrm{L}}$ and $\theta_{\tau}$ were $61 \pm 7$ and $22 \pm 5$ $\mathrm{cm} / \mathrm{s}$ before freezing and $63 \pm 8$ and $21 \pm 3 \mathrm{~cm} / \mathrm{s}$ after freezing.

The activation pattern of the left ventricular free wall was recorded with a spoonshaped mapping electrode molded to the epicardial surface of the heart, containing 240 individual silver leads (diameter $0.3 \mathrm{~mm}$, spacing $2.25 \mathrm{~mm}$ ). A computerized mapping 


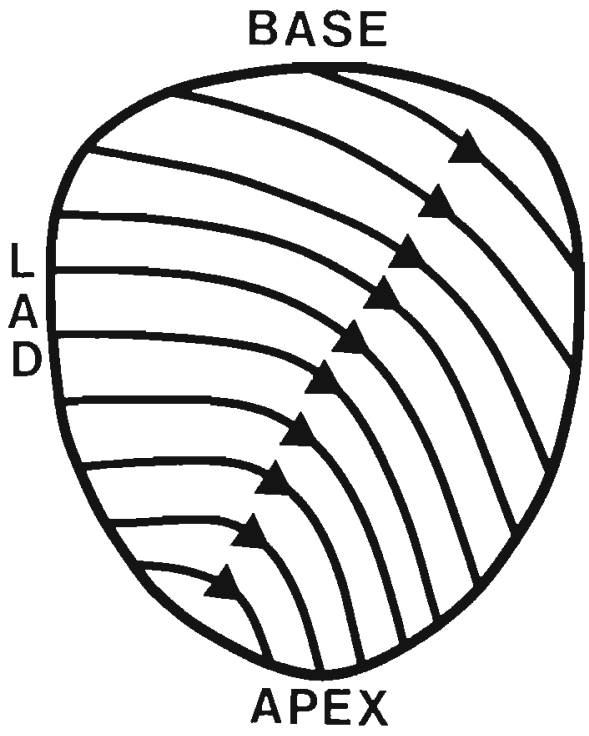

Figure 2.3: Schematic drawing of the epicardial fiber orientation of the left ventricular free wall. Close to the $L A D$ the long fiber axis is parallel to the Atrio-ventricular ring while at the center and the right side of the free wall the fiber axis curves towards the apex. $L A D=$ left anterior descending artery.

system $^{20}$ was used to construct activation maps (See chapter 3). Figure 2.5 shows examples of the activation patterns during sinus rhythm and during regular pacing after the cryoprocedure and during pacing after freezing. During sinus rhythm (left map) at intervals between 300 and $400 \mathrm{~ms}$ the earliest epicardial activation occurred at the center of the free wall and at the apex. Large areas of the left ventricular free wall were activated almost simultaneously and the total epicardial activation time was about $25 \mathrm{~ms}$. During regular pacing (intervals of $250 \mathrm{~ms}$ ) from a pacing site close to the LAD (middle panel) the epicardial activation sequence was different than during sinus rhythm. The impulse now spread radially from the site of stimulation and during the first $30 \mathrm{~ms}$ the isochrones had a regular ellipsoid shape, caused by the anisotropic structure of the myocardium. Conduction was fast parallel to the fiber orientation and much slower in a transverse direction (See chapter 5). However, already at a short distance from the pacing site conduction transverse to the fiber axis was bypassed by faster depolarization waves from deeper layers of the myocardium. Thus, after the first $30 \mathrm{~ms}$ the remaining part of the left ventricle was activated almost simultaneously

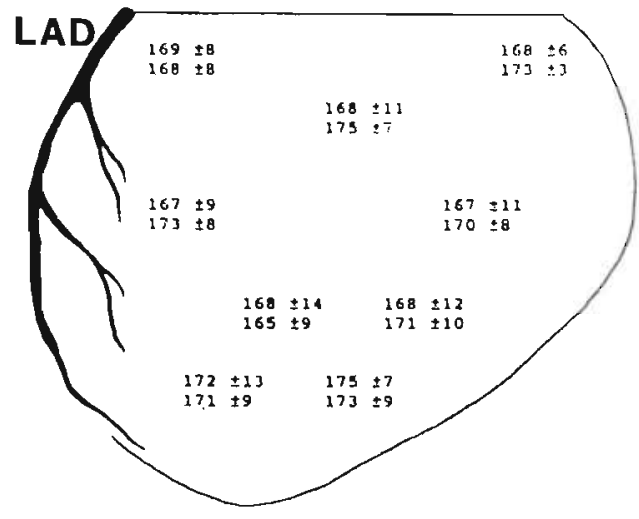

Figure 2.4: Average values of refractory periods as obtained in 10 hearts at 9 different epicardial sites before and after freezing. Top numbers represent average values before freezing while bottom numbers were obtained after the cryoprocedure. At none of the epicardial sites was the refractory period altered significantly by the freezing technique. $L A D=L$ Left Anterior Descending artery. (Reproduced with permission from Schalij et al. Am J Physiol 1992;263:H1466H1478) 

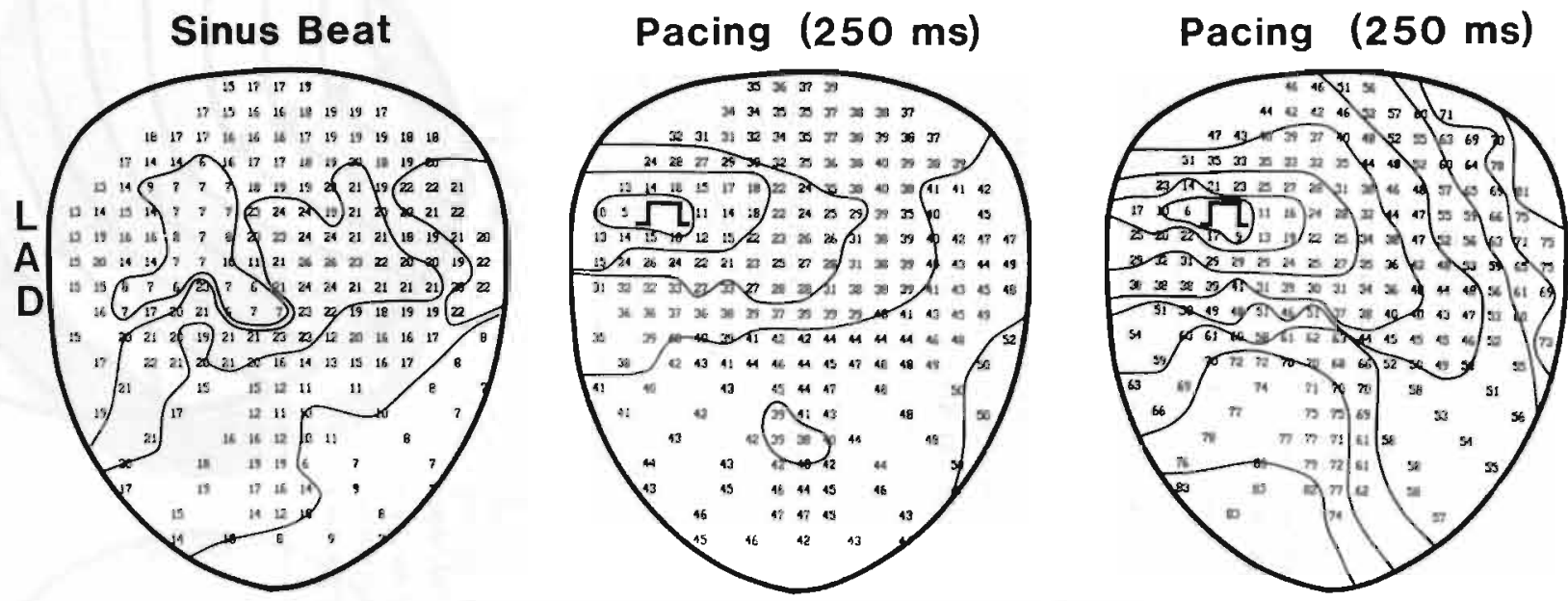

Figure 2.5: Three activation maps of left ventricular free wall during sinus rhythm and regular pacing (250 ms intervals) before cryoprocedure and during regular pacing after cryoprocedure. During sinus rhythm (left map) two sites of earliest epicardial activation were observed at the center of the free wall and the apex. The remaining parts of the left free wall were activated almost simultaneously, due to breakthrough of depolarizing waves from deeper layers. During regular pacing (intervals of $250 \mathrm{~ms}$ ) before the cryoprocedure (middle map) a uniform anisotropic spread of activation occurred from site of stimulation. After $30 \mathrm{~ms}$ the ellipsoid shape of the isochrones was lost because the slow transwerse conduction was bypassed by breakthrough of faster endocardial wave fronts. After the cryoprocedure the spread of activation was still uniform. The ellipsoid shape of the isochrones was more apparent than before freezing, because slow transverse conduction was no longer bypassed by faster intramural depolarization waves. Activation times are given in milliseconds and isochrone lines are drawn at 10 ms interoals. See text for description. 
again, leading to a total epicardial activation time of about $52 \mathrm{~ms}$.

The cryoprocedure not only destructed $4 / 5$ th of the ventricular myocardium but also the ventricular conduction system, rendering the left ventricle quiescent unless stimulated. After freezing pacing at $250 \mathrm{~ms}$ intervals (figure 2.5, right map) still resulted in a smooth spread of activation without any apparent gross disturbances in conduction, indicating that the uniform anisotropic tissue structure was preserved. Due to the removal of the endocardial and intramural muscle layers, slow wave fronts transverse to the epicardial fiber orientation were no longer bypassed by faster intramural depolarization waves and thus proceeded over a greater distance.

Therefore, even far away from the pacing site the ellipsoid shape of the isochrones was preserved and the total activation time of the epicardial surface of the left ventricle was increased to $83 \mathrm{~ms}$.

\section{Summary}

A cryoprocedure was devised to create a model of a Langendorff-perfused twodimensional layer of uniform anisotropic left ventricular subepicardium. ${ }^{12,13}$ Both the refractory refractory periods and their dispersion and the longitudinal and trans-verse conduction velocities were not affected by the cryoprocedure. However, transverse conduction was now able to proceed over a greater distance since it was no longer bypassed by faster intramural depolarization waves. This preparation thus provides a good 2-D experimental model to study anisotropic impulse propagation and arrhythmias. 


\section{REFERENCES}

1. Sommer J, Johnson E. Uitrastructure of cardiac muscle. In: Handbook of Physiology sect. II, vol. I, The Heart. Eds. Berne R, Sperelakis N, Geiger S. Bethesda, American Physiological Society, 1980:130-186

2. Clerc L. Directional differences of impulse spread in trabecular muscle from mammalian heart. J Physiol 1976;255:335-346

3. Dräper M, Mya-Tu M. A comparison of the conduction velocity in cardiac tissues of various mammals. Quart J Exptl Physiol 1959;44:91-109

4. Sano T, Takayama N, Shimamoto T. Directional difference of conduction velocity in the cardiac ventricular syncytium studied by micorelectrodes. Circ Res 1959;7:262-268

5. Spach M, Miller W, Geselowitz D, Barr R, Kootsey J, Johnson E. The discontinuous nature of propagation in normal canine cardiac muscle. Evidence for recurrent discontinuities of intracellular resistance that affect the membrane currents. Circ Res 1981;48:35-54

6. Spach M, Kootsey J, Sloan J. Active modulation of electrical coupling between cardiac cells of the dog. A mechanism for transient and steady-state variations in conduction velocity. Circ Res 1982;51:347-362

7. Spach M, Dolber P, Heidlage J, Kootsey J, Johnson E. Propagating depolarization in anisotropic human and canine cardiac muscle: apparent directional differences in membrane capacitance. $\mathrm{A}$ simplified model for selective directional effects of modifying the sodium conductance on Vmax, Tfoot, and the propagation safety factor. Circ Res 1987;60:206-219

8. Spach M, Dolber P, Heidlage J. Influence of the passive anisotropic properties on directional differences in propagation following modification of the sodium current in human atrial muscle. A model of reentry based on anisotropic discontinuous propagation. Circ Res 1988;62:811-832

9. Spach $M$, Dolber $P$, Heidlage F. Interaction of inhomogeneities of repolarization with anisotropic propagation in dog atria. A mechanism for both preventing and initiating reentry. Circ Res $1989 ; 65: 1612-1631$

10. Streeter DD: Gross morphology and fiber geometry of the heart. In Berne R, Sperilakis N, Geiger $S$ (eds): Handbook of physiology. Bethesda, American Physiological Society, 1979, pp 61-112

11. Schalij MJ. Anisotropy and ventricular tachycardia. Doctoral Thesis, University of Limburg, Maastricht, The Netherlands 1988.

12. Schalij M, Lammers W, Rensma P, et al. Anisotropic conduction and reentry in perfused epicardi um of rabbit left ventricle. Am J Physiol 1992;263:H1466-H1478

13. Allessie M, Schalij M, Kirchhof C, Boersma L, Huybers M, Hollen J. Experimental electrophysiology and arrhythmogenicity: Anisotropy and ventricular tachycardia. Eur Heart J 1989;10:E2-E8

14. Brugada J, Boersma L, Kirchhof $C$, Brugada P, Havenith M, Wellens H, Allessie M. Double-wave reentry as a mechanism of acceleration of ventricular tachycardia. Circulation 1990;81:1633-1643

15. Hill B, Hunt A, Courtney K. Reentrant tachycardia in a thin layer of ventricular subepicardium: Effects of d-Sotalol and Lidocaine. J Cardiovasc Pharmacol 1990;16:871-880

16. Nassif G, Dillon S, Rayhill S, Wit A. Reentrant circuits and the effects of heptanol in a rabbit mod el of infarction with a uniform anisotropic epicardial border zone. J Cardiovasc Electrophysiol 1993;4:112-133

17. Fishbein M, Meerbaum S, Rit J, Lando U, Kanmatsuse, Mercier J, Corday E, Ganz W. Early phase acute myocardial infaret size quantification: validation of the triphenyl tetrazolium chloride tissue enzyme staining technique. Am Heart J 1981;101:593-600

18. Nachlas M, Shnitka T. Macroscopic identification of early myocardial infarcts by alterations in dehydrogenase activity. Am J Pathol 1963;42:379-385

19. Allessie M, Hoeks A, Schmitz G, Reneman R. On-line mapping system for the visualization of the electrical activation of the heart. Int J Card Imaging 1986;2:59-63 


\section{CHAPTER 3}

\section{ANISOTROPIC REENTRY IN VENTRICULAR MYOCARDIUM.}

Characteristics of the line of functional block during ventricular tachycardia.

Lucas Boersma

Martin Jan Schalij

Maya Huyberts

Charles Kirchhof

Maurits Allessie

In preparation 


\section{INTRODUCTION}

Histologic and electrophysiologic studies have shown that after myocardial infarction, ventricular arrhythmias may arise from thin layers of surviving myocardium overlying the infarcted tissue. ${ }^{1-4}$ Mapping studies in canine models of infarction have revealed that such arrhythmias are based on functional reentrant circuits, either in a figure-of-eight pattern with two wavefronts circulating around two separate lines of functional conduction block with a central common pathway ${ }^{5-8}$ or as a single circulating wave. Dillon et al. $^{7}$ have shown that tissue anisotropy in the border zone was of major influence on the course of functional reentry. During initiation of VT the line of conduction block was perpendicular to the fiber orientation while during sustained VT the central line of functional conduction block was oriented parallel to the fiber orientation. $^{\text {? }}$

To further examine the influence of tissue anisotropy on conduction disturbances and reentry, a model of Langendorff-perfused thin layers of rabbit left ventricular subepicardium was developed, ${ }^{9}$ exhibiting clear directional differences in conduction velocity due to the anisotropic properties of the myocardium. In this preparation, ventricular tachycardias can be induced based on reentry around a line of functional conduction block oriented parallel to the epicardial fiber orientation. ${ }^{9,10}$ In the present investigation, we studied the characteristics of these anisotropic reentrant circuits with high resolution extracellular mapping and micro-electrode impalements.

\section{METHODS}

\section{Preparation}

In 11 Langendorff-perfused rabbit hearts, a thin layer of left ventricular subepicardium was created by a cryoprocedure. The perfusion system and the cryoprocedure have been described in detail in chapter 2 .

\section{Recording and Stimulation}

A spoon-shaped mapping electrode, molded to the epicardial surface of the left ventricle and containing 240 silver electrodes (diameter $0.3 \mathrm{~mm}$, interelectrode spacing $2.25 \mathrm{~mm}$ ) was used to map the ventricular activation. The on-line mapping system used for recording and analysis of the data has been described in chapter 2 . In the activation maps that were constructed, conduction block was defined as a time delay of more than $20 \mathrm{~ms} / \mathrm{mm}$ (apparent conduction velocity $<5 \mathrm{~cm} / \mathrm{s}$ ).

A computer-controlled stimulator (Medtronic SP3084) was used for bipolar stimulation at any pair of electrodes selected from the mapping electrode. The inducibility of ventricular arrhythmias was tested with programmed electrical stimulation (4 $\mathrm{x}$ diastolic threshold) with up to 2 extrastimuli and incremental pacing both before and after the cryoprocedure. The extrastimuli were applied after a train of 15 basic stimuli at an interval of $300 \mathrm{~ms}$. The coupling interval between the last basic stimulus (S1) and the first extrastimulus (S2) was decreased with steps of $2 \mathrm{~ms}$ until an arrhythmia was induced or the extrastimulus no longer elicited a propagated response. In that case, the S1-S2 interval was increased with $5 \mathrm{~ms}$ and a second extrastimulus (S3) was applied. For $\mathrm{S} 3$ the same sequence of stimulation as for $\$ 2$ was repeated.

Intracellular action potentials were recorded with standard potassium-filled ( $3 \mathrm{M})$ glass 
micro-electrodes. The signals were pre-amplified and displayed on an oscilloscope (Tektronix $5103 \mathrm{~N}$ ) and stored on videotape. During sustained VT action potentials were recorded sequentially. The activation time at an impalement site was determined by comparing the moment of local depolarization with a fixed extracellular reference electogram. Due to the slight movement of the heart during VT the microelectrode was immediately broken during an impalement. Therefore, all action potential were recorded with a microelectrode with a broken tip, which markedly reduced the amplitude of the recordings.

\section{RESULTS}

\section{Inducibility of Ventricular Arrhythmias}

In 30 hearts programmed electrical stimulation with up to two extrastimuli and rapid pacing was performed to determine the inducibility of ventricular arrhythmias. Before the cryoprocedure, rapid pacing induced ventricular fibrillation in all hearts, as shown in the upper tracing of figure 3.1. After the cryoprocedure, ventricular fibrillation could no longer be induced in any of the hearts. Instead, rapid pacing now initiated sustained monomorphic VT in 13 of 30 hearts (middle tracing), while in 17 hearts short episodes of non-sustained VT were induced (lower tracing). During steady-state, all sustained VTs had a regular cycle length of $109 \pm 10 \mathrm{~ms}(n=13)$ with a variation in interval of $2 \pm 1$ $\mathrm{ms}$ over a period of 10 beats. The episodes of non-sustained VT had a mean duration of $40 \pm 63$ seconds. During each episode marked variations in cycle length occurred. The average of the mean cycle length in 17 episodes of non-sustained VT was $108 \pm 4 \mathrm{~ms}$ with an average maximal range of $16 \pm 2 \mathrm{~ms}$.

BEFORE CRYOPROCEDURE

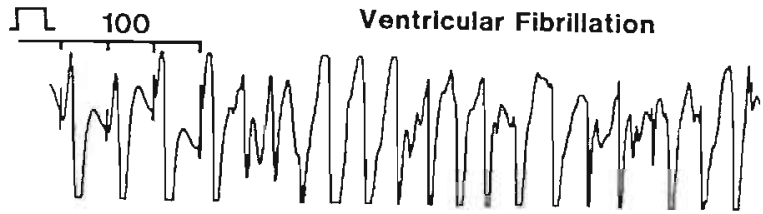

AFTER CRYOPROCEDURE

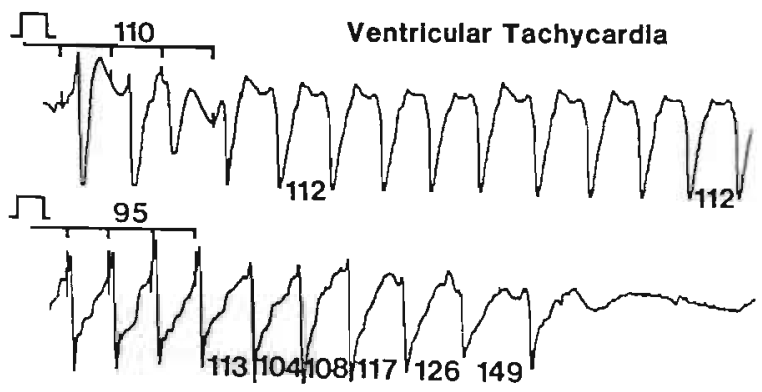

Figure 3.1: Three tracings showing the effects of rapid pacing before and after the cryoprocedure. Before the cryoprocedure (upper tracing) rapid pacing induced ventricular fibrillation. After the cryoprocedure, rapid pacing initiated either sustained (middle tracing) or nonsustained (lower tracing) episodes of ventricular tachycardia. See text for discussion. 

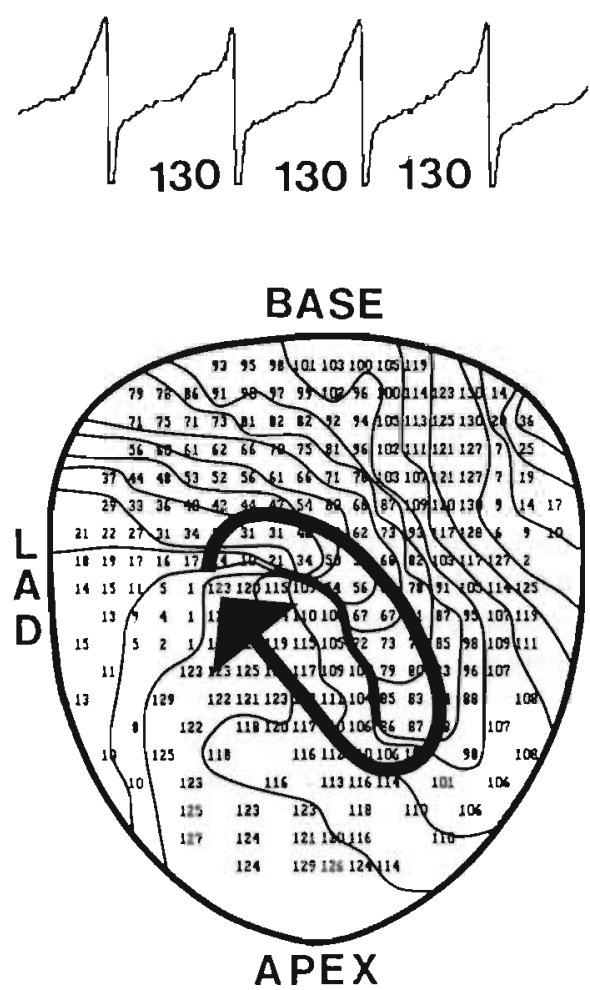

Figure 3.2: Extracellular activation map of reentrant ventricular tachycardia (VT). VT u'as based on a clockwise circulating wave around a line of functional conduction block with a cycle length of $130 \mathrm{~ms}$ (top tracing). The line of block was oriented parallel to the epicardial fiber orientation. Along both the long limbs of the circuit conduction was fast $(36$ and $50 \mathrm{~cm} / \mathrm{s})$ while at the pivot points crowding of the isochrones occurred and conduction was markedly slower (9 and $10 \mathrm{~cm} / \mathrm{s})$. Activation times are given in milliseconds and isochrone line's are drawn at $10 \mathrm{~ms}$ intervals. Conduction block is indicated by a thick line. $L A D=$ Left Anterior Descending artery.

Characteristics of Sustained Monomorphic Ventricular Tachycardia around a line of Functional Conduction Block

After endocardial freezing of the left ventricle, pacing at long coupling intervals resulted in a uniform anisotropic spread of activation without any gross conduction disturbances (see chapter 2). At shorter pacing intervals, lines of functional conduction block appeared and in 13 hearts resulted in initiation of sustained monomorphic VT., 910 Figure 3.2 shows an example of a sustained clockwise functional VT. The circulating wave propagated around a central line of functional conduction block with a revolution time of $130 \mathrm{~ms}$. Along the long limbs of the circuit parallel to the line of block, the circulating wave propagated at a relatively high speed $(36$ and $50 \mathrm{~cm} / \mathrm{s}$ respectively) while at the pivoting points the conduction velocity was markedly slower (10 and 9 $\mathrm{cm} / \mathrm{s}$ ). Fast conduction was due to propagation along the epicardial fiber axis while slow conduction was due to tranvserse impulse propagation (chapter 2).

To study the conduction properties at the center of the anisotropic reentry circuit, this area was extensively mapped with micro-electrodes. Figure 3.3 shows the local activation times at about 100 sites of impalement during the same VT as in figure 3.2. The action potentials were recorded sequentially at regular distances of $0.5 \mathrm{~mm}$ over a total area of 4 by $6 \mathrm{~mm}$. Conduction block was defined as a time delay of more than $10 \mathrm{~ms}$ over $0.5 \mathrm{~mm}$ (apparent conduction velocity $<5 \mathrm{~cm} / \mathrm{s}$ ). By this criterion the line of conduction block was Z-shaped and seemed to be confined to a small area of only 2.0 by $4.5 \mathrm{~mm}$. This contrasted markedly with the extracellular activation map, showing a straight line of block in an area of 6.75 by $9.0 \mathrm{~mm}$. Also, in the extracellular activation 


\section{BASE}

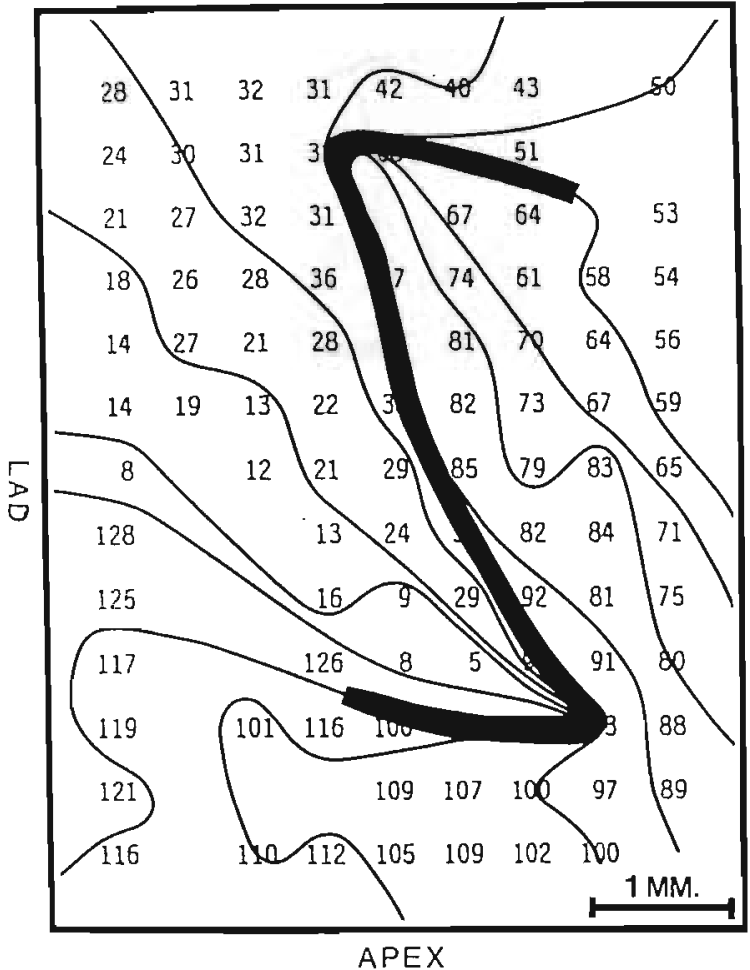

Figure 3.3: Activation map of the central area of block during the same clockwise VT as in figure 2 based on sequential intracellular mapping. Activation times were determined at each site of a micro-electrode impalement. The impalement sites were 0.5 $\mathrm{mm}$ apart. The central line of conduction block was Z-shaped. Note that the isochrone lines are almost parallel to the line of block. Activation times are given in milliseconds and isochrone lines are drawn at $10 \mathrm{~ms}$ intervals. Conduction block is indicated by a thick line. $L A D=$ Left Anterior Descending artery. See text for discussion.

map in both long limbs of the circuit the isochrones were perpendicular to the line of block, while in the intracellular map the isochrones were oriented almost parallel to the line of block.

The left panel in figure 3.4 shows the action potentials that were recorded across the central line of block, while the right panel shows the action potentials on the central line of block. The amplitude of all action potentials is low, probably because the recording microelectrode had a broken tip. In the left panel the action potentials at sites 1-3 and 7-8 at both sides of the line of block show a steep upstroke and a complete repolarization, indicated by a clear diastolic interval between subsequent activations. However, at sites 4-6 close to the line of block the action potentials have two major components with a slow upstroke and no phase 4 . During a single revolution the circulating wave approached the tissue under these electrodes twice from an opposite side. The right panel of figure 3.4 shows that all the electrograms on the line of block had such a double component. The phase difference between both components was largest at the middle of the central line of block but diminished towards both ends of the line of block. Due to the double frequency of activation, the cells at the central line of block were continuously depolarized above their resting membrane potential, preventing generation of a full-blown local action potential.

Figure 3.5 shows the intracellular action potentials that were recorded at the perpendicular extensions at both ends of the central line of block. The left panel shows 

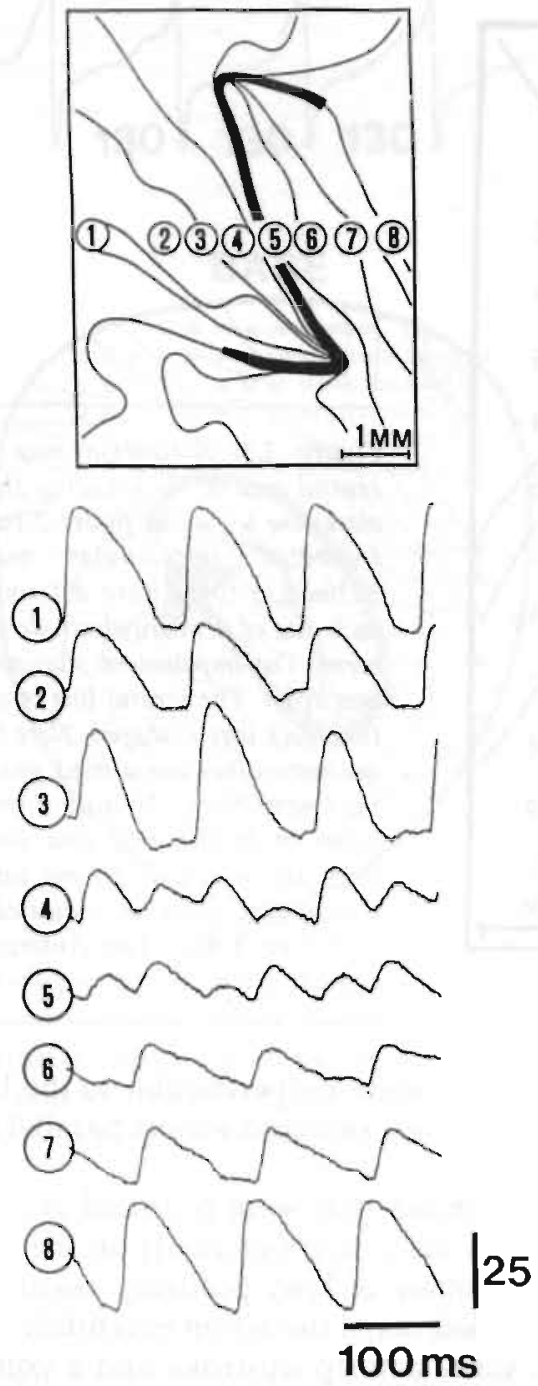
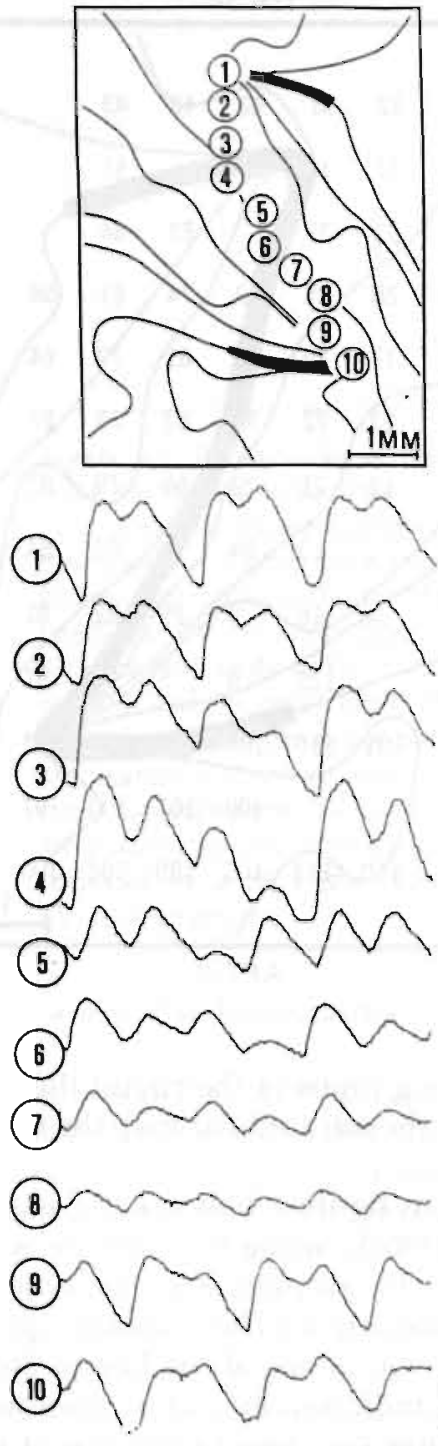
$25 \mathrm{mV}$

\section{$100 \mathrm{~ms}$}

Figure 3.4: Left: Action potentials recorded across the central line of block during the same VT as in figure 3.3. The lacalization of the sites of recording is indicated in the isochrone map at the top. At a distance from the line of block the action potentials 1-3 and 7-8 were of subnormal amplitude but had a regular morphology. Close to the central line of block the electrograms 4-6 had a very small amplitude and multiple components during one action potential. Right: Action potentials recorded on the central line of conduction block during the same VT as in figure 3.3. The localization of the sites of recording is indicated in the isochrone map at the top. The 10 action potentials were of subnormal amplitude and showed two major components during each revolution of the circulating VT wave. See text for discussion. 


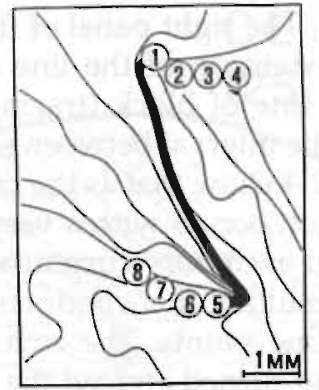

(1) $V$ R
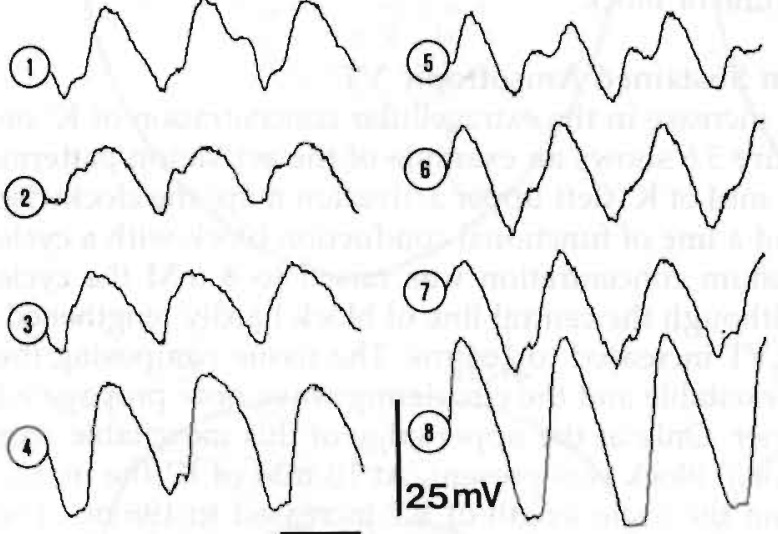

$\overline{100 \mathrm{~ms}}$
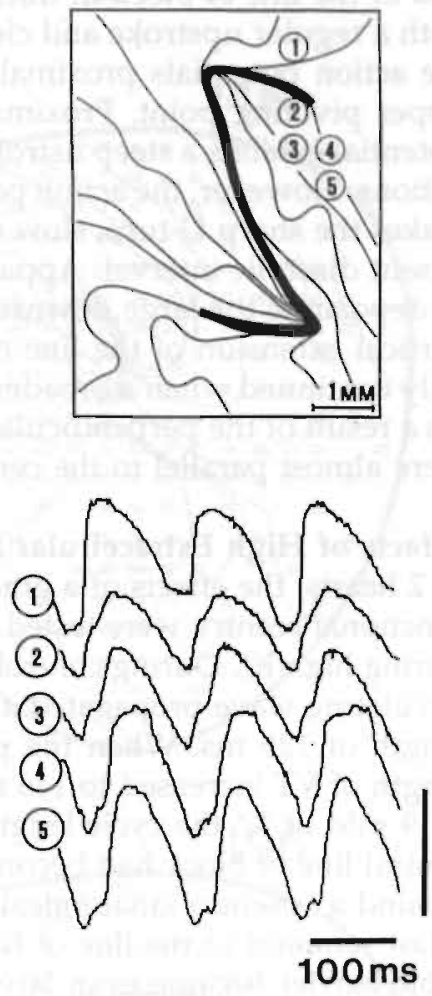

Figure 3.5: Left: Action potentials recorded on the perpendicular extensions of the central line of conduction block during the same VT as in figure 3.3. The localization of the sites of recording is indicated in the isochrone map at the top. Electrograms 1-3 and 5-7 were of subnormal amplitude and showed a foot-potential with a delay in repolarization. Right: Action potentials recorded across the perpendicular extensions of the central line of block during the same VT as in figure 3.3. The localization of the sites of recording is indicated in the isochrone map at the top. All the electrograms were of subnormal amplitude. Proximal to the line of block the shape of the action potential (tracing 1) was normal while just distal to the line of block (tracings 2 and 3) a foot-potential and a delay in repolarization occurred. Away from the line of block the action potential shape became regular again. Vertical calibration bar $=25$ milliVolts. See text for discussion.

the electrograms located on the line of block at the pivoting point. Electrograms 1 and 5 at the end of the central line of block show an electrotonic hump superimposed on the repolarization, annihilating phase 4 of the action potential. The action potentials in tracings 2-4 and 6-8 exhibit a foot-potential superimposed on the beginning of the upstroke. Close to the central line of block in tracings 2 and 6 , this foot-potential was big and there was no apparent diastolic interval between subsequent activations. At the 
end of the line of block in tracings 4 and 8 , the action potentials became normal again with a regular upstroke and clear diastolic interval. The right panel of figure 3.5 shows the action potentials proximal and distal to the extension of the line of block at the upper pivoting point. Proximal to the horizontal line of block (tracing 1) the action potential exhibits a steep ustroke and a clear diastolic interval between subsequent activations. However, the action potentials in tracings $2-4$ show that as the circulating wave makes the sharp U-turn, slow steplike depolarization occurs with a very small or even absent diastolic interval. Apparently, the generated excitatory current was insufficient to depolarize the large downstream tissue mass, resulting in a conduction delay and a vertical extension of the line of block at the pivoting points. The circulating impulse only continued when a broader part of the wave had turned around the pivoting point. As a result of the perpendicular block lines, the isochrones distal to the pivoting points were almost parallel to the central line of block.

\section{Effects of High Extracellular $\mathrm{K}^{+}$on Sustained Anisotropic VT}

In 2 hearts, the effects of a gradual increase in the extracellular concentration of $\mathrm{K}^{+}$on functional reentry were tested. Figure 3.6 shows an example of the activation patterns during high $\mathrm{K}^{+}$. During control at $4 \mathrm{mM}$ of $\mathrm{K}^{+}$(left upper activation map) the clockwise circulating wave propagated around a line of functional conduction block with a cycle length of $125 \mathrm{~ms}$. When the potassium concentration was raised to $8 \mathrm{mM}$ the cycle length of VT increased to $146 \mathrm{~ms}$ although the central line of block hardly lengthened. At $9 \mathrm{mM}$ of $\mathrm{K}^{+}$the cycle length of VT increased to $164 \mathrm{~ms}$. The tissue composing the central line of block had become inexcitable and the circulating wave now propagated around a "pseudo"-anatomical barrier. Only at the upper edge of this inexcitable area a last remnant of the line of functional block was present. At $10 \mathrm{mM}$ of $\mathrm{K}^{+}$the inexcitable barrier became even larger and the cycle length of VT increased to $196 \mathrm{~ms}$. The original line of functional conduction block had completely disappeared. At this moment in time the potassium infusion was stopped since VT was no longer of a functional nature. In the other heart the effects of high extracellular $\mathrm{K}^{+}$on VT were similar. The central line of conduction block first lengthened slightly during a gradual raise in $\mathrm{K}^{+}$, but at higher concentrations the tissue under the line of block became inexcitable without leading to teimination of VT.

\section{Interval Variations during Non-Sustained Functional VT}

In 17 hearts rapid pacing after the cryoprocedure could only induce non-sustained episodes of functional VT. Figure 3.7 shows an example of the activation pattern and the electrograms during an episode of non-sustained VT. The average cycle length during this episode of non-sustained VT was $106 \mathrm{~ms}$ (range 105-119 ms). The four activation maps show that VT was based on a clockwise circulating wave around a central line of functional conduction block. This line of block seemed to be stable at the center of the free wall of the left ventricle. More detailed examination of the line of block revealed that during subsequent beats minute changes occurred, both in the localization and the length of the arc of block. At the right, electrograms A-I recorded around the line of block show that the slight changes of the line of block coincided with beat-to-beat variations in interval in each electrogram. Despite these interval variations the morphology of each electrogram during subsequent acitvations was almost the same because the general direction of propagation of the circulating wave did not change. 

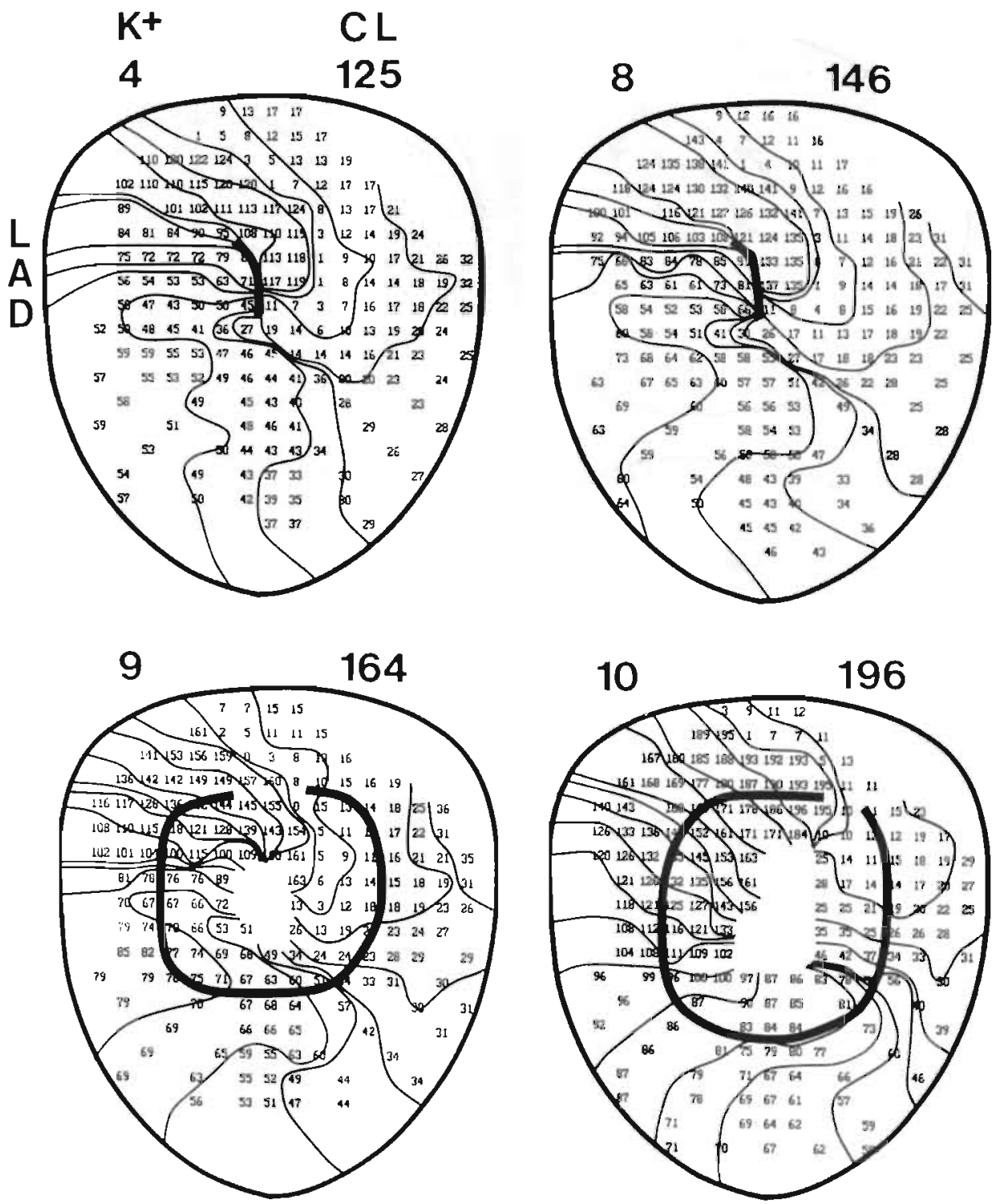

Figure 3.6: Four activation maps showing the effetts of a gradual raise in extracellular $K^{+}$concentration on functional VT. During control (4 $\mathrm{mM}$ of $\left.\mathrm{K}^{*}\right)$ VT with a cycle length of $125 \mathrm{~ms}$ was based on a clockwise circulating wave around a line of functional conduction block. During an increase in extracellular $K^{*}$ to 8 , 9 , and $10 \mathrm{mM}$ the cycle length of VT increased to 146,164 , and $196 \mathrm{~ms}$ respectively. VT remained based on a clockwise circulating wave but the line of functional conduction block was transformed into an area of inexcitability. Activation times are given in milliseconds and isochrone line are drawn at $10 \mathrm{~ms}$ intervals. Conduction block is indicated by a thick line. $K^{+}=$extracellular concentration of $K^{*}, C L=$ cycle length of VT. $L A D=$ Left Anterior Descending artery. See text for discussion. 

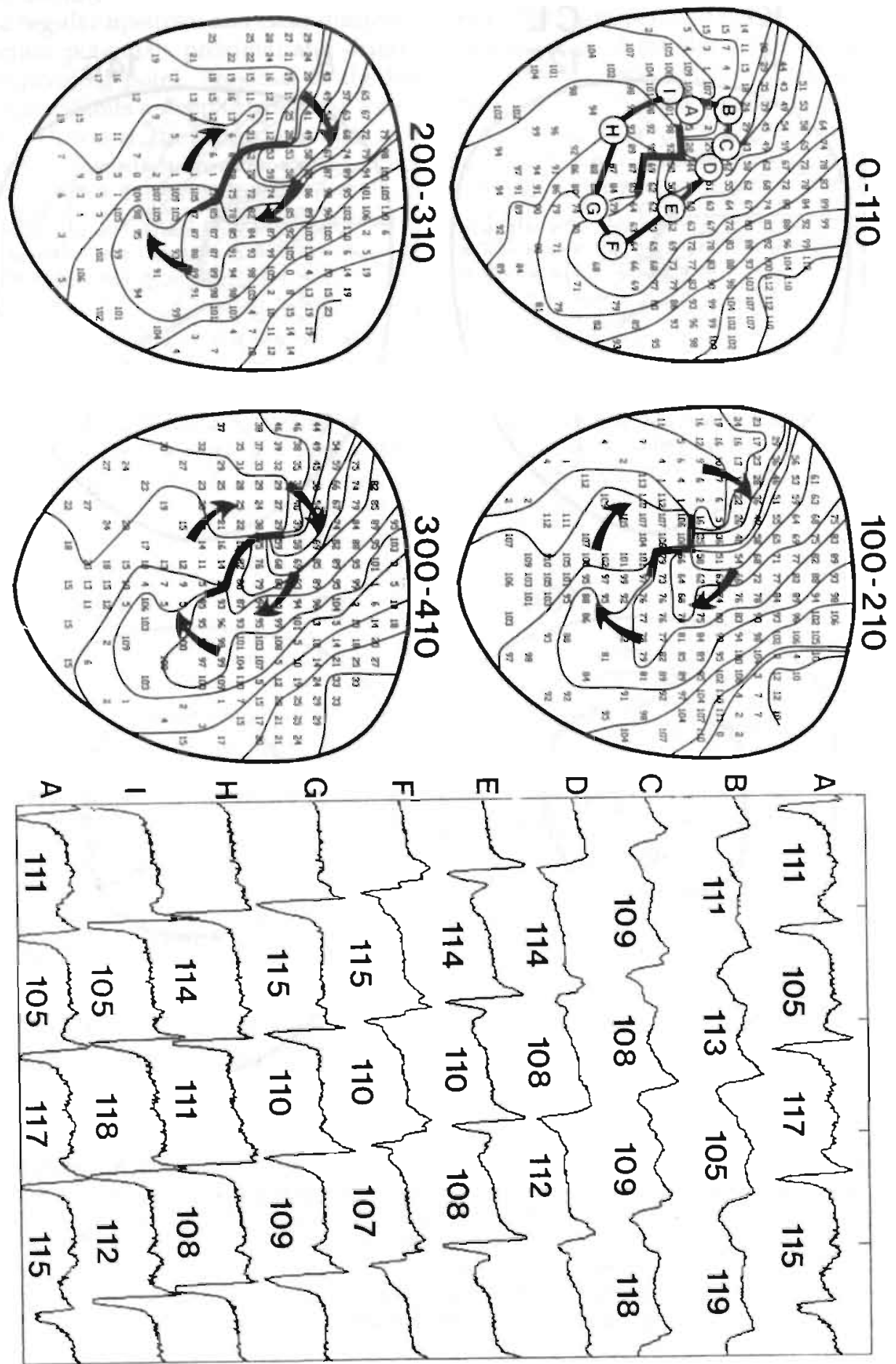
Figure 3.7 (left): Left: Four activation maps during an episode of non-swstained VT. VT was based on a clockwise circulating wave around a central line of functional conduction block. The subsequent activation maps have an overlap of $10 \mathrm{~ms}$. During each of the four beats the central line of block remained located in. the same area of the free wall, but the size of the line of block changed minutely from beat to beat. Activation times are given in milliseconds and isochrone lines are drawn at $10 \mathrm{~ms}$ interoals. Conduction block is indicated by a thick line. Right: Nine electrograms recorded at sites A-I indicated in the upper left activation map. The cycle length of the consecutive beats varied markedly but tine electrogram morphology remained more or less the same. See text for discussion.

\section{Termination of Non-Sustained Ventricular Tachycardia.}

Figure 3.8 gives an example of termination of non-sutained VT. The four activation maps show the last $400 \mathrm{~ms}$ of the same episode of clockwise VT as in figure 3.7. Three beats prior to termination (upper left map) the reentrant circuit was still located at the center of the free wall. Hoever, during the next revolution the circulating wave could short-circuit the lower end of the central line of block. As a result, the line of block lengthened at the opposite pivoting point, shifting the circuit upwards (upper right panel). When the circulating wave turned around the upper pivoting point, the line of block again changed position and extended even further upward to the base (lower left map), and as a result the circulating wave could short-circuit the lower end of the line of block again. However, during the next beat the upper end of the line of block extended up to the boundary of the ventricle at the base, resulting in annihilation of the circulating wave.

Although termination of VT was most commonly due to conduction block of the circulating wave at the boundary of the ventricle, we also found a second mechanism for termination of functional VT. Figure 3.9 shows an example. This episode of nonsustained VT lasted only 5 seconds and the average cycle length of VT was $101 \mathrm{~ms}$ (range 89-125 ms). VT was based on a circulating wave propagating in a clockwise: direction around a small line of functional block located at the center of the free wall (upper left panel). Two beats prior to termination the line of block had shifted upwards to the base (upper right panel). As the circulating wave propagated around the line of block, its offspring propagated towards the apex as a broad planar wave. However, at the center of the free wall a line of conduction block split this broad wave in two parts that propagated in opposite directions to collide with each other at the other side of the line of block. During the next beat (lower left panel) the reentrant circuit at the base had extended even further upward to the boundary, resulting in annihilation of the circulating wave. The last offspring of the circulating wave again propagated towards the apex as a broad planar wave. However, the localization of the line of block had changed slightly its length had increased and in contrast to the previous beat, the two waves that turned around this line of block did not annihilate each other. Instead, the two waves fused and propagated slowly towards the center of the line of block where breakthrough occurred and a completely new figure-of-eight reentry circuit was initiated. During the subsequent beat (lower right panel) two circulating waves propagated in opposite direction around the line of block. However, both waves arrived early at the central common pathway where the cells had not yet recovered their excitability, resulting in conduction block and termination of VT. 

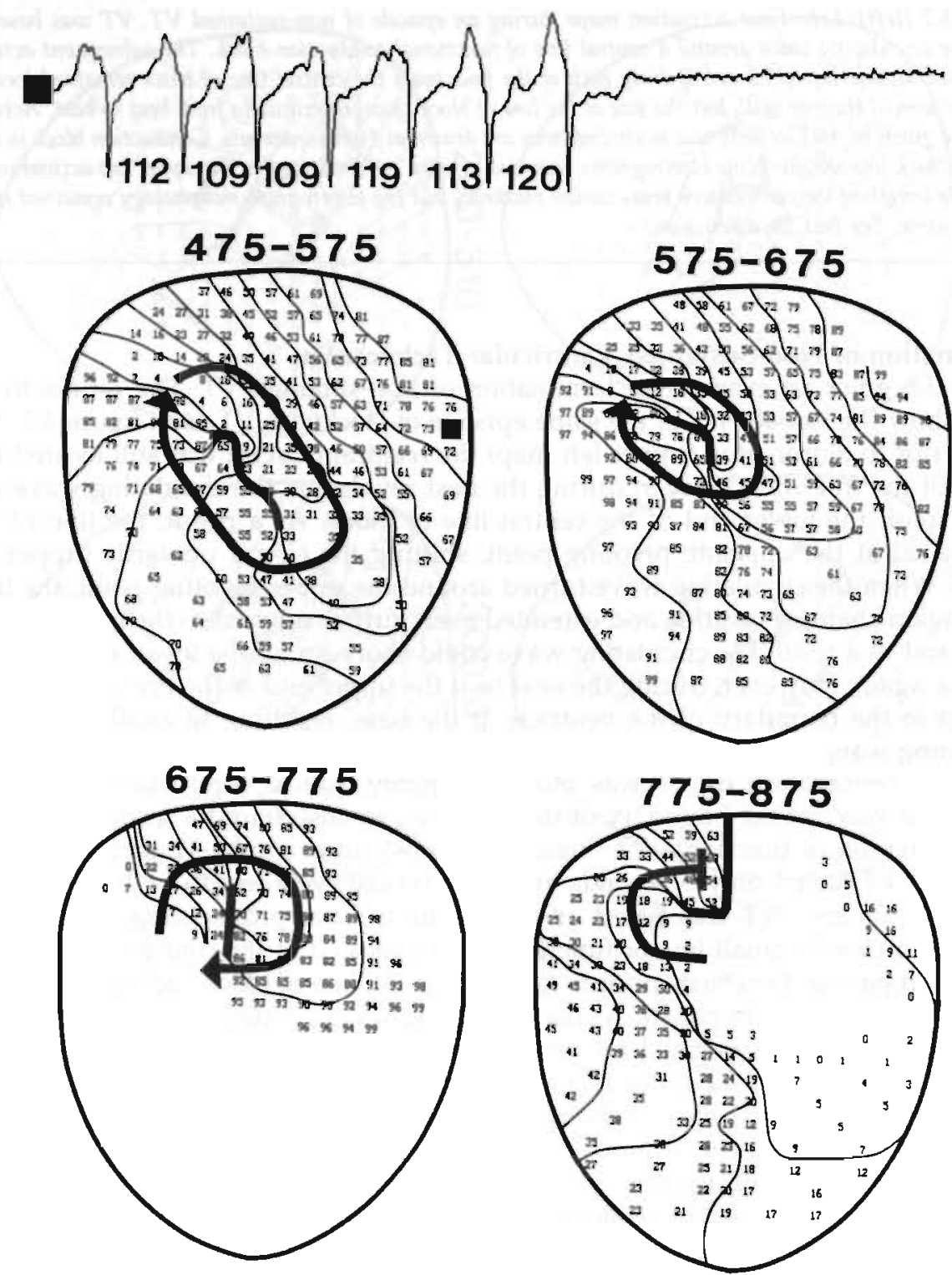

Figure 3.8: Four consecutive maps showing the activation patterns preceding spontaneous termination of the same episode of non-sustained VT as in figure 3.7. Three beats before termination (upper left map) the reentrant circuit was located at the center of the lef ventricular free wall. During the following beats the central line of functional block shifted upward. When it extended to the boundary of the ventricle at the base the circulating wave was annihilated and VT was terminated. Activation times are given in milliseconds and isochrone lines are drawn in milliseconds. Activation times are given in milliseconds and isochrone lines are drawn at $10 \mathrm{~ms}$ intervals. Conduction block is indicated by a thick line. See text for discussion. 

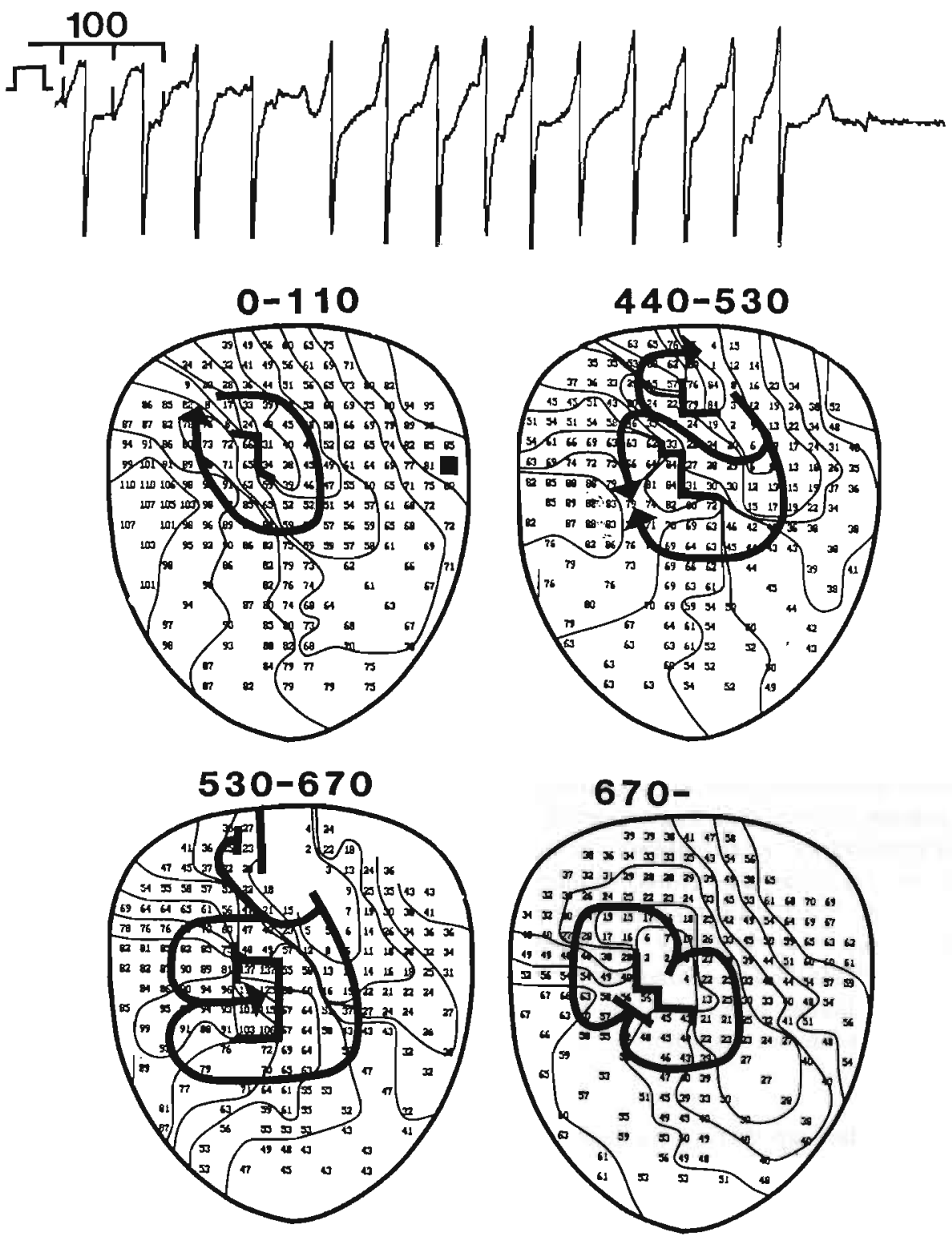

Figure 3.9: Spontaneous termination of an episode of non-sustained VT. The reentrant circuit was located at the center of the left ventricular free wall (upper left map). During the two subsequent beats (upper right, lower left), the main reentrant circuit shifted towards the boundary where it was annihilated. The last actioation wave that was initiated by this circuit was split in two parts that turned around a long line of intraventricular conduction block. Subsequently, both waves fused and reentered the line of block (lower right map), initiating a figure-eight reentrant circuit with two circulating waves propagating in opposite direction around separate lines of block. Conduction block in the central common pathway finally terminated VT. Activation times are given in milliseconds and isochrones are drawn at $10 \mathrm{~ms}$ intervals. Conduction block is indicated by thick line. See text for discussion. 


\section{DİSCUSSION}

Experimental studies have provided evidence that VT after myocardial infarction is based on reentrant excitation ${ }^{3,5,6,7,9}$ in thin epicardial or endocardial surviving sheets of muscle overlying the healed infarct. ${ }^{11-13}$ In this so-called border zone, during the first week after ischemia excitability thresholds and refractoriness as well as their spatial dispersion are increased, ${ }^{2}$ and clear directional differences in propagation are present., ${ }^{4,14}$ Action potential disorders usually return to normal within two weeks to two months after the ischemic event ${ }^{4}$ but scar formation and separation of adjacent muscle bundles by fibrotic tissue disrupt the normal uniform anisotropic tissue structure, resulting in slow discontinuous impulse propagation. ${ }^{4,7,14}$ Dillon et al ${ }^{7}$ and Cardinal et al. ${ }^{8}$ postulated that the non-uniform anisotropic tissue structure of the border zone in canine infarcts was of major importance for the characteristics of functional VT. They showed that during initiation of functional VT the lines of conduction block were perpendicular to the epicardial fiber orientation, which was attributed to a lower safety factor for conduction in the longitudinal direction. ${ }^{15,16}$ During sustained VT the central line of functional block was usually oriented parallel to the epicardial fiber orientation. Several investigators provided evidence that dispersion in refractoriness may be a key element for the initiation and perpetuation of functional reentry in the border zone. It was shown ${ }^{17}$ that within the border zone a marked dispersion in refractoriness exists, and that sympathetic stimulation creates a large disparity in refractoriness between normal myocardium and the denervated border zone, ${ }^{18}$ providing an alternative expalnation for the occurrence of reentry in the border zone. Studies from our own laboratory show that functional reentry can also occur in isolated Langendorff perfused sheets of rabbit subepicardium. ${ }^{9,10,19}$ Schalij et al. ${ }^{19}$ showed that during slow pacing in this preparation, the spatial dispersion in refractoriness was very small and probably of minor importance for the inducibility of VT. The functional circuits that can be induced in our model of normal anisotropic myocardium exhibit similar characteristics as the anisotropic circuits in the border zone described by Dillon et al. ${ }^{7}$ and Cardinal et al. ${ }^{8}$, with a central line of block that is usually oriented parallel to the epicardial fiber orientation. Thus, tissue anisotropy seems to be a key factor in the course of functional VT in thin layers myocardium. However, spatial dispersion in refractoriness may contribute to the development of clinical VTs in the border zone of myocardial infarcts in humans.

\section{The Excitable Gap during Sustained Anisotropic Reentrant Circuits}

In our experiments, anisotropic reentry was only sustained in $43 \%$ of the hearts. In general, the stability of a reentrant arrhythmia depends on the existence of an excitable gap in the circuit. Several investigators have reported an excitable gap during functional reentry in anisotropic myocardium, ${ }^{9,10,19-22}$ although its origin is not well understood. In the present study, micro-electrode recordings showed that during anisotropic VT the tissue at the line of block was continuously in a partly depolarized state while the action potentials outside the center of the functional reentrant circuit had a clear diastolic interval between subsequent activations, indicating the presence an excitable gap in the circuit. There are several explanations for the existence of this excitable gap that must be considered.

1) It has been shown that due to a mismatch between the generated excitatory current and the current load imposed on a propagating wavefront, ${ }^{15,16}$ slowing of conduction 
and conduction block can occur while the cells are readily excitable. At the pivoting points of an anisotropic reentrant circuit such a mismatch may occur when the circulating wave makes a sharp U-turn and encounters the large current load of the wellcoupled cells of the longitudinal limb of the circuit. Conduction will momentarily be halted until more excitatory current is provided when a larger part of the circulating wavefront has turned around the pivoting point, creating an excitable gap at other sites of the circuit. In our micro-electrode studies we found that the action potentials at the pivoting points all showed a steplike depolarization, providing evidence that such a delay in conduction indeed occurs at the pivoting points of an anisotropic circuit.

2) Spatial dispersion in refractoriness may contribute to the formation of an excitable gap. It has been shown that the repolarization of cells may be prolonged by electrotonic interaction with more remote depolarizing tissue. ${ }^{23} \mathrm{~A}$ similar situation occurs at the pivoting points of a functional circuit, where depolarization of the cells distal to the pivoting points may prolong repolarization at the proximal side. We observed that the action potentials at the pivoting points of the circuit did not exhibit a clear phase 4, indicating a prolongation in repolarization which in turn leads to an excitable gap at other sites in the circuit.

3) Micro-anatomical discontinuities or areas with a reduced safety factor for conduction may create an excitable gap during anisotropic VT and will also provide an anchoring point for the reentrant circuit. In our experiments, during slow pacing conduction was uniform without gross conduction disturbances while during rapid pacing arcs of functional block appeared, providing a barrier around which sustained anisotropic reentry could be started. When the extracellular concentration of $\mathrm{K}^{+}$was raised during functional VT the tissue at the line of block was the first to become inexcitable. This may suggest that the area where functional block occurs already has a lower safety factor for conduction during control, and therefore may serve as an anchoring point for a reentrant circuit. Nassif et al. ${ }^{24}$ have adopted our model and observed that anisotropic reentry was only sustained when the cryoprocedure had left a very thin epicardial layer. This raises the question whether in such thin epicardial layers small microanatomical discontinuities created by the cryoprocedure may contribute to the excitable gap and the stability of anisotropic VT. In our experiments, about $57 \%$ of the functional VTs was non-sustained which according to Nassif et al. ${ }^{24}$ may be explained by a thicker epicardial layer. Small discontinuities in conduction at the epicardial surface, providing potential anchoring sites for functional reenty, may become insigni-ficant because they are bypassed by depolarizing wavefronts in deeper layers.

Two possible mechanisms for spontaneous termination of non-sustained VT were found. In the majority of cases functional VT was based on a single circulating wave, which was linked to a specific site in the ventricle without stabilizing. A short-circuiting of the central line of block by the circulating wave initiated a shift of the reentrant circuit away from its original location. Termination occurred when the central line of block extended to the boundary of the left ventricle and the circulating wave was annihilated. Pertsov et al. ${ }^{22}$ have previously shown that drifting spiral waves in isolated strips of 2-D myocardium were also terminated by annihilation at the boundary of the preparation. It remains to be determined whether this mechanism applies to termination of reentrant VT in the whole heart, since even in the infarcted 3-D left ventricle a fixed boundary may not be present. VT after experimental myocardial infarction is often based on figure-of-eight reentry. We found that in case of figure-of-eight reentry, termination was due to block of the circulating waves in the central common pathway, 
similar to previous findings of Mehra et al. ${ }^{6}$.

\section{Anisotropic Reentry as a Mechanism for Clinical VT}

Several experimental studies have shown that an epicardial or endocardial border zone overlying the infarct ${ }^{11-13}$ is the source for functionally determined reentrant arrhythmias. ${ }^{3.5-8}$ Dillon et al. ${ }^{7}$ and Cardinal et al. ${ }^{8}$ found that the non-uniform anisotropic myocardial structure of this border zone ${ }^{4,14}$ greatly influenced the characteristics of the reentrant circuit. Both in present and previous studies ${ }^{9,10,19}$ from our laboratory, it has been shown that also in non-diseased anisotropic myocardium functional reentrant circuits can be initiated. It remains to be determined whether clinical VT after myocardial infarction is based on functional anisotropic reentry. de Bakker et al. ${ }^{25,26}$ have shown that in human infarcted hearts macro-reentrant pathways exist, consisting of zones of normal myocardium connected by thin strands of surviving muscle bundels in infarcted regions. This corresponds very well with the reset characteristics of clinical VTs, demonstrating a fully excitable gap within the circuit. ${ }^{27}$ Analysis of the reset characteristics of experimental models of functional and anatomical VT may shed more light on the nature of the reentrant circuits in patients.

Treatment of clinical VTs with antiarrhythmic drugs is only succesfull in about $40 \%$ of patients, ${ }^{28}$ while proarrhythmic side-effects are not uncommon. Several investigators have shown that non-sustained VTs in patients can become sustained during antiarrhythmic drug treatment. ${ }^{27}$ In a previous study from our laboratory, Brugada et al. ${ }^{30}$ provided experimental evidence for such a pro-arrhythmic effect by showing that flecainide transformed episodes of non-sustained anisotropic VT into sustained anisotropic VT. Several observations suggest that it may be difficult to cure anisotropic VT with antiarrhythmic drugs. In the present study, depression of the active membrane properties by high concentrations of extracellular $\mathrm{K}^{+}$resulted in only a moderate slowing of VT, while at higher concentrations of $\mathrm{K}^{+}$functional VT was not terminated but merely transformed into a stable anatomic VT. Previously, Brugada et al ${ }^{30}$ and Nassif et al. ${ }^{24}$ have demonstrated that moderate concentrations of flecainide and heptanol also moderately slowed functional VTs without terminating the arrhythmia. These results may explain why in a specific subset of patients anti-arrhythmic drugs are not successfull in the treatment of VT.

\section{CONDENSED ABSTRACT}

In thin layers of anisotropic epicardium, rapid pacing induced episodes of sustained and non-sustained ventricular tachycardia (VT) around a line of functional conduction block. During sustained VT the cycle length was regular and the reentrant circuit was located at a fixed site of the ventricle. Microelectrode recordings during VT showed that the circulating wave was delayed by a local slowing of depolarization and a prolongation of repolarization at the turning point of the functional reentry circuit. Due to this lical delay at the pivotting points, an excitable gap was present in all other parts of the reentrant circuit. Elevation of the extracellular $\mathrm{K}^{+}$concentration to $10 \mathrm{mM}$ moderately increased the cycle length of ventricular tachycardia without resulting in termination. Instead, during high $\mathrm{K}^{+}$the central line of functional block became inexcitable, transforming the functional circuit into a more stable anatomical reentrant circuit. During non-sustained VT large variations in cycle length occurred due to small changes 
in size of the funcional circuit. spontaneous termination of non-sustained VT predominantly occurred by a sudden shift of the circuit to the boundary of the ventricle were it was annihilated. In some cases, termination of VT was due to block in the central common pathway of a new figure-of-eight reentry circuit. 


\section{REFERENCES}

1. Josephson M, Horowitz L, Farshidi A, Kastor J. Recurrent sustained ventricular tachycardia: 1 . Mechanisms. Circulation 1978;57:431-439

2. Michelson E, Spear J, Moore E. Electrophysiologic and anatomic correlates of sustained ventricular tachyarrhythmias in a model of chronic myocardial infarction. Am J Cardiol 1980;45:583-590

3. Wit A, Allessie M, Bonke F, Lammers W, Smeets J, Fenoglio J. Electrophysiologic mapping to determine the mechanism of experimental ventricular tachycardia initiated by premature impulses. Experimental approach and initial results demonstrating reentrant excitation. Am J Cardiol 1982;49:166-185

4. Ursell P, Gardner P, Albala A, Fenoglio J, Wit A. Structural and electrophysiological changes in the epicardial border zone of canine myocardial infarcts during infarct healing. Circ Res 1985;56:436-451

5. El-Sherif N, Smith A, Evans K. Canine ventricular arrhythmias in the late myocardial infarction period. 8. Epicardial mapping of reentrant circuits. Circ Res 1981;49:255-265

6. Mehra R, Zeiler R, Gough W, et al. Reentrant ventricular arrhythmias in the late myocardial infarction period. 9. Electrophysiologic anatomic correlation of reentrant circuits. Circulation 1983;67:11-24

7. Dillon S, Allessie M, Ursell P, Wit A. Influences of anisotropic tissue structure on reentrant circuits in the epicardial border zone of subacute canine infarcts. Circ Res 1988;63:182-206

8. Cardinal $R$, Vermeulen $M$, Shenasa $M$, et al. Anisotropic conduction and functional dissociation of ischemic tissue during reentrant ventricular tachycardia in canine myocardial infarction. Circulation 1988;77:1162-1176

9. Schalij M, Lammers W, Rensma P, et al. Anisotropic conduction and reentry in perfused epicardium of rabbit ventricle. Am J Physiol 1992;263:H1466-H1478

10. Allessie M, Schalij M, Kirchhof C, Huyberts M, Boersma L, Hollen J. Experimental electrophysiology and arrhythmogenecity. Anisotropy and ventricular tachycardia. Eur Heart J 1989;10:E8-E14

11. Horowitz L, Josephson M, Harken A. Epicardial and endocardial activation during sustained ventricular tachycardia in man. Circulation 1980;61:1227-1238

12. Garan H, Fallon J, Rosenthal S, Ruskin J. Endocardial, intramural, and epicardial activation patterns during sustained monomorphic ventricular tachycardia in late canine myocardial infarction. Circ Res 1987;60:879-896

13. Littmann L, Svenson R, Gallagher J, Selle J, Zimmern S, Fedor J, Colavita: Functional role of the epicardium in postinfarction ventricular tachycardia. Observations derived from computerized epicardial activation mapping, entrainment, and epicardial laser photoablation. Circulation 1991;83:1577-1591

14. Richards D, Blake G, Spear J, Moore E. Electrophysiologic substrate for ventricular tachycardia: correlation of properties in vivo and in vitro. Circulation 1984;69:369-381

15. Spach M, Miller W, Geselowitz D, et al. The discontinuous nature of propagation in normal cardiac muscle. Evidence for recurrent discontinuities of intracellular resistance that affect the membrane currents. Circ Res 1981;48:35-54

16. Spach $M$, Miller W, Dolber $P$, et al. The functional role of structural complexities in the propagating depolarization in the atrium of the dog. Cardiac conduction disturbances due to discontinuities of effective axial resistivity. Circ Res 1982;50:175-191

17. Gough W, Mehra R, Restivo M, Zeiler R, El-Sherif N. Reentrant ventricular arrhythmias in the late myocardial infarction period in the dog. 13. Correlation of activation and refractory maps. Circ Res $1985 ; 57: 432-442$

18. Butrous $G$, Gough W, Restivo M, Yang H, El-Sherif N. Adrenergic effects on reentrant ventricular tachycardia in subacute myocardial infarction. Circulation 1992;86:247-254

19. Schalij M. Anisotropy and ventricular tachycardia. Thesis, University of Limburg, Maastricht, The Netherlands, 1988

20. Allessie M, Schalij M, Kirchhof C, Boersma L, Huyberts M, Hollen J: Electrophysiology of spiral waves in two dimensions: the role of anisotropy. Ann NY Acad Sci 1990;591:247-256

21. Wit A, Dillon S. Anisotropic reentry. In: Cardiac Electrophysiology. From cell to bedside. Eds. Zipes D, Jalife J, WB Saunders Company, 1990:353-363 
22. Pertsov A, Davidenko J, Salomonsz R, Baxter W, Jalife J. Spiral waves of excitation underie reentrant activity in isolated cardiac muscle. Circ Res 1993;72:631-650

23. Osaka T, Kodama I, Tsuboi N, Toyama J, Yamada K. Effects of activation sequence and anisotropic cellular geometry on the repolarization phase of action potential of dog ventricular muscles. Circulation 1987;76:226-236

24. Nassif G, Dillon S, Rayhill S, Wit A. Reentrant circuits and the effects of heptanol in a rabbit model of infarction with a uniform anisotropic epicardial border zone. J Cardiovasc Electrophysiol 1993;4:112-133

25. de Bakker J, Van Capelle F, Janse $M$, et al. Reentry as a cause of ventricular tachycardia in patients with chronic ischemic heart disease: electrophysiologic and anatomic correlation. Circulation 1988;77:589-606

26. de Bakker J, Coronel R, Tasseron S, Wilde A, Opthof T, Janse M, van Capelle, Becker A, Jambroes $G$. Ventricular tachycardia in the infarcted Langendorff-perfused human heart: role of the arrangement of surviving cardiac fibers. J Am coll Cardiol 1990;15:1594-1607

27. Almendral J, Stamato N, Rosenthal M, Marchlinski F, Miller J, Josephson M. Resetting response patterns during sustained ventricular tachycardia: relationship to the excitable gap. Circulation 1986a;74:722-730

28. Marchlinski F. Ventricular tachycardia: clinical presentation, course, and therapy. In: Cardiac Electrophysiology. From cell to bedside. Eds. Zipes D, Jalife J, WB Saunders Company, 1990:756777

29. Buxton A, Josephson M, Marchlinski F, Miller J. Polymorphic ventricular tachycardia induced by programmed electrical stimulation: response to procainamide. J Am Coll Cardiol 1993;21:90-98

30. Brugada J, Boersma L, Kirchhof C, Allessie M. Proarrhythmic effects of Flecainide. Experimental evidence for increased susceptibility to reentrant arrhythmias. Circulation 1991;84:1808-1818 


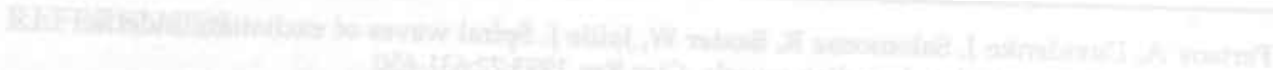

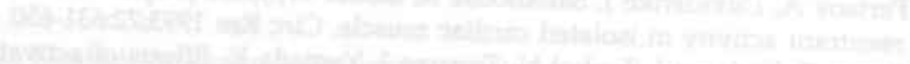

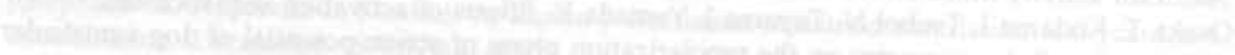

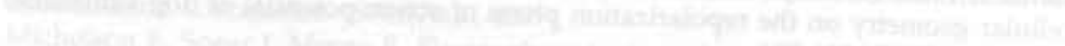

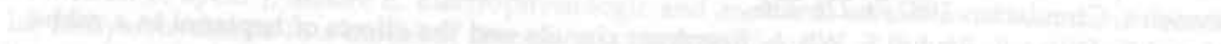

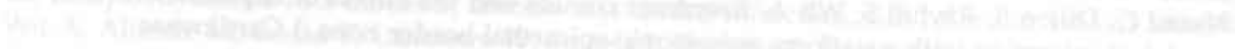
The

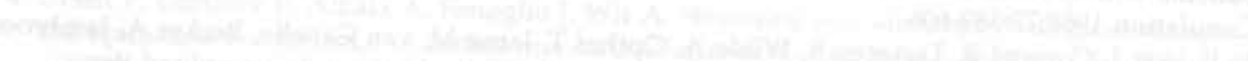


CHAPTER 4

REENTRANT EXCITATION AROUND A FIXED ANATOMICAL OBSTACLE IN UNIFORM ANISOTROPIC VENTRICULAR MYOCARDIUM.

Josep Brugada

Lucas Boersma

Charles Kirchhof

Vivian Heynen

Maurits Allessie

Circulation 1991;84:1296-1306 


\section{INTRODUCTION}

For reentrant excitation to occur, unidirectional block at some site within the myocardium and conduction over an alternative pathway are necessary conditions.' Cardiac tissue has a different axial resistance along and perpendicular to the fiber axis of the myocardial fibers, which results in direction-dependent differences in conduction velocity (anisotropic conduction). ${ }^{2}$ Spach et al. ${ }^{3}$ postulated that anisotro-py affects not only conduction velocity but also the safety factor for conduction defined as the ratio between the amount of current generated by the cells at the crest of the depolarization wave and the amount of current required to excite the surrounding cells. The safety factor was supposed to be lower during fast longitudi-nal conduction because the lower axial resistance imposes a larger electrotonic load on the depolarization wave.

The same authors ${ }^{3}$ also demonstrated in canine atrial myocardium that propagation could continue in a direction tranverse to the long fiber axis while block occurred in the longitudinal direction. Both of the two necessary conditions for reentry therefore might be provided by tissue anisotropy: 1) lowering of the stimulating efficacy of the depolarization wave might cause conduction block in a longitudinal direction, and 2) slow conduction transverse to the fiber direction might provie sufficient delay to allow recovery of excitability of cells proximal to the site of block. The aim of the present study was to investigate the significance of tissue anisotropy for initiation of reentrant ventricular tachycardia (VT) and define the relative roles of fiber orienta-tion and spatial dispersion in refractory periods.

\section{METHODS}

\section{Preparation}

The complete experimental set-up has been described in chapter 2 . In brief, in 20 Langendorff-perfused rabbit hearts a thin layer of left ventricular subepicardium was created by a cryoprocedure. In this thin layer of perfused anisotropic tissue, an obstacle was created by applying an epicardial cryoprobe of $20 \times 2 \mathrm{~mm}$ at a distance of $10 \mathrm{~mm}$ of the left anterior descending artery (LAD). This produced an area of approximately $25 \times 10 \mathrm{~mm}$ where the thin epicardial layer was destroyed with surviving myocardium all around it. Thus, the final preparation consisted of a ring of perfused epicardial tissue surrounding a non-excitable barrier. According to the normal course of the fiber orientation in the epicardium of the left ventricle, impulse conduction around this ring was perpendicular to the fiber axis in the "corridor" between the LAD and the obstacle and parallel to the fiber orientation at the base and upper part of the free wall of the left ventricle.

\section{Recording and Stimulation}

For high resolution mapping of epicardial excitation, a spoon-shaped electrode containing 240 individual silver electrodes (diameter $0.3 \mathrm{~mm}$ ) at regular distances of $2.25 \mathrm{~mm}$. The characteristics of the mapping system used for recording and analy-zing data has been previously described. ${ }^{4,6}$ Programmed electrical stimulation was performed with a programmable constant current stimulator delivering square pulses of $2 \mathrm{~ms}$ duration at twice diastolic threshold for regular pacing and fourfold diastolic threshold for premature stimuli. Any pair of electrodes from the "spoon" electrode could be 
selected for stimulation.

Determination of the effective refractory period (ERP) was performed by the extrastimulus technique. After 10 basic driven beats at $250 \mathrm{~ms}$ interval, one extrastimulus was introduced at a decreasing coupling interval until the premature stimulus failed to excite the ventricle. The longest premature interstimulus interval that was not followed by ventricular activation was taken as the ERP. If reentrant VT was not initiated during measurement of the refractory period the inducibility of VT was determined in the following manner. The coupling interval of the first extrastimulus was set at $5 \mathrm{~ms}$ longer than the ERP and a second extrastimulus was introduced with decreasing coupling intervals until ventricular activation failed or reentrant VT occurred. If the latter was not the case the complete sequence was repeated while introducing a third extrastimulus. The experimental protocol included determination of the ERP at 16 sites evenly distributed around the obstacle, determination of inducibility of reentrant VT around the obstacle at these 16 sites, and identification of the site of unidirectional block initiating VT.

\section{Measurement of Conduction Velocities}

Conduction velocity was calculated as the distance travelled normal to the isochrones per unit time. The three areas in which conduction velocity was measured in each experiment were the corridor between the LAD and the obstacle (conduction transverse to the fiber orientation), the basal part of the left free wall (conduction parallel to the fiber orientation), and the site at which uni-directional block occurred. Conduction velocity could be accurately measured at all sites around the obstacle except for the apex. The complex organization of fiber orientation in the apex made it very difficult to precisely follow the pathway of the activation wave in this area and relate it to the fiber orientation. Consequently, measurements of conduction velocity were considered not to be reliable in this area.

\section{Histological Examination}

To verify the effects of the freezing procedure, after the experiment 10 of the hearts were cut into $2 \mathrm{~mm}$ thick sections parallel to the atrioventricular ring from base to apex and stained with a solution of nitroblue tetrazolium. ${ }^{7}$ In the remaining hearts, sections of tissue of approximately $20 \times 20 \mathrm{~cm}$ cuit parallel to the epicardial surface were obtained at different sites in the ventricle. After reticulum staining ${ }^{8}$ micruscopic examination of the fiber orientation at these sites was performed.

\section{Determination of fiber Orientation and Direction of Impulse Propagation}

Fiber orientation was determined by light microscopy at different sites in each section while the observer was blinded to the position of the section in the ventricle (see Figure 4.1 for an example). In this case four slices of tissue around the obstacle (dashed area in the center) were studied. Fiber orientation was measured at distances of approximately $5 \mathrm{~mm}$ (thick lines) and the angle between these lines and the atrioventricular ring was determined. To analyze consistency between different experiments, fiber orientation was determined at eight segments defined around the ring. From the activation maps the direction of propagation of the impulse was determined by drawing a continuous arow normal to the isochrones around the obstacle. The angle between the direction of propagation and the atrioventricular ring was determined at different sites around the obstacle by an observer blinded to the histological results. The difference 


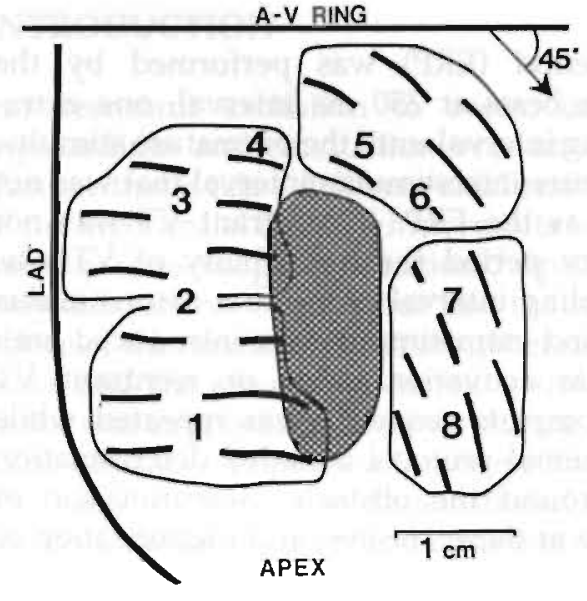

FIBER ORIENTATION
Figure 4.1: Schematic drawing showing the determination of the fiber orientation. Four histological sections obtained at different locations around the obstacle (crosshatched area in the center) are represented. Fiber orientation was measured at approximately 5-mm intervals (thick lines). Angle between these lines and the atrioventricular ( $A V$ ) ring was calculated. Numbers indicate eight segments defined to compare fiber orientation between different experiments. $L A D=$ Left Anterior Descending coronary artery.

between both measurements (fiber orientation and direction of activation in relation to the atrioventricular ring) yielded the angle between fiber orientation and direction of propagation of the impulse. An angle of 0 degrees indicates that the impulse propagated parallel to the fiber axis; at an angle of 90 degrees the impulse propagated perpendicular to the fiber orientation.

\section{RESULTS}

Histological Characterization of Tissue

Histological examination showed that the right ventricular interventricular septum and endocardial and intramural layers of the left ventricle were completely destroyed by the cryoprocedure. The surviving epicardial layer was $1.2 \pm 0.2 \mathrm{~mm}$ thick. In a given heart slight variation in the thickness of the surviving epicardium was present (range 0.9-1.3 $\mathrm{mm}$ ) with a thinner surviving layer at sites covered by large vessels of epicardial fat. At the area where the epicardial obstacle was created by cryocoagulation a complete transmural lesion was present. The size of the lesion was $25 \pm 3 \times 8 \pm 2 \mathrm{~mm}$ and it was located parallel to the LAD. Surviving ventricular epicardial tissue was observed all around the obstacle. Histological determination of fiber orientation showed a consistent orientation of fiber direction around the ring in different experiments (Table 4.1). In the corridor between the LAD and the obstacle the fibers ran parallel to each other in a direction corresponding to the AV-ring. In the free wall of the left ventricle the fibers curved gradually from base to apex.

\section{Electrophysiologic Characterization of the Preparation}

Pacing at different sites around the obstacle showed that in 17 of 20 experiments the impulse propagated smoothly in two directions around the obstacle with fast conduction at the basal part of the left ventricle $(62 \pm 6 \mathrm{~cm} / \mathrm{s})$ and slow conduction at the corridor between the LAD and the obstacle $(27 \pm 4 \mathrm{~cm} / \mathrm{s})$. In figure 4.2 an example is given during regular pacing (S1) at the base of the left ventricle. One wave front 


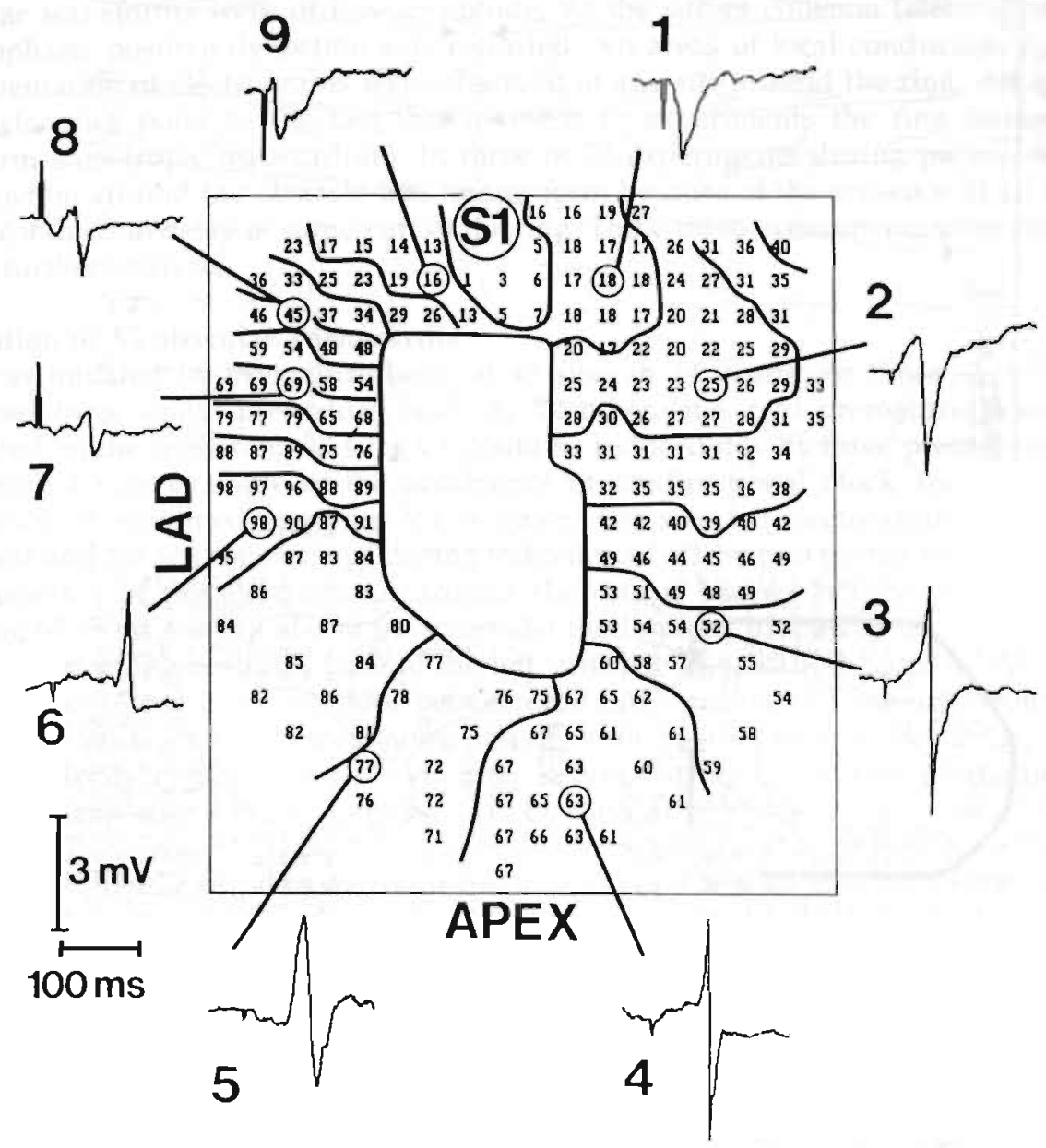

Figure 4.2: Actioation map of epicardial ring in left ventricle during regular pacing (S1) at $250 \mathrm{~ms}$ intoroals at base of left ventricle. Numbers indicate local activation times in $\mathrm{ms}$. Isochrones are dracun at $10 \mathrm{~ms}$ intervals. Empty area in center represents inexcitable lesion created by cryoprocedure. Nine selected electrograms together with their locations around the ring are shown. See text for description.

propagated counterclockwise through the corridor and another wave front was conducting clockwise through the free wall of the left ventricle. The two wave fronts collided $98 \mathrm{~ms}$ after the stimulus was given. Because conduction in the corridor transverse to fiber orientation was slow the site of collision was not opposite to the site of pacing but instead was closer to the segment of slow conduction.

The configuration of the extracellular wave forms differed depending on the site of recording. Near the site of stimulation (electrograms 1 and 9) the wave forms showed a negative monophasic deflection. During longitudinal conduction (electrograms 2-4) the electrograms were biphasic of high amplitude and exhibiting a sharp negative 


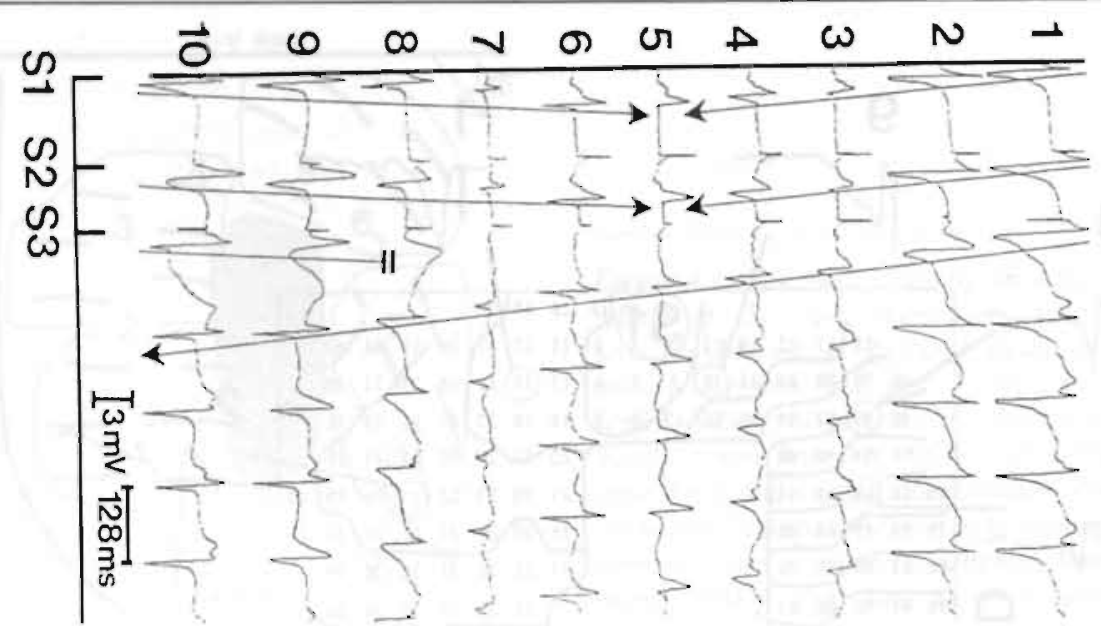

LAD
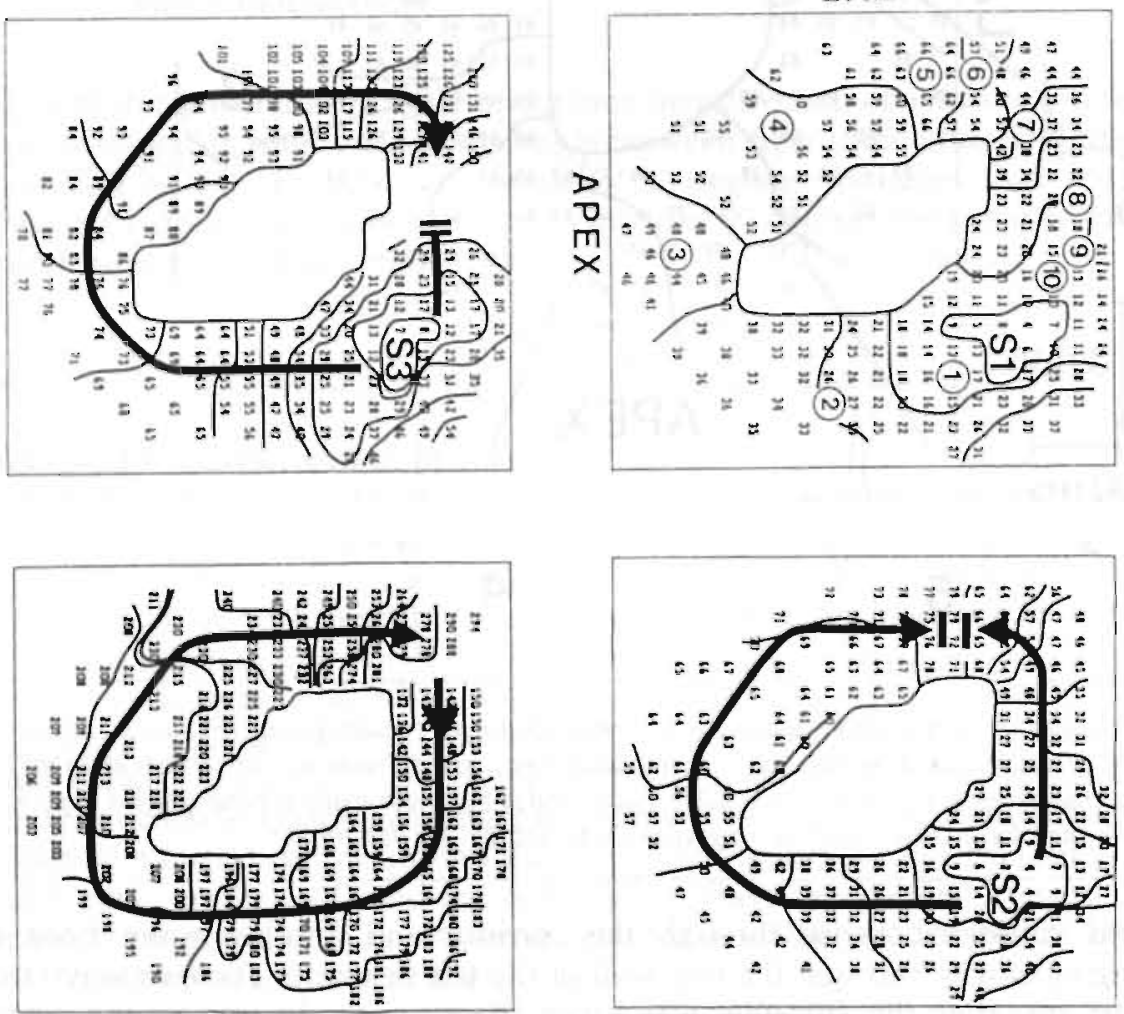

Figure 4.3: Ten selected electrograms around obstacle and 4 activation maps during initation of VT. S1 last of a series of 10 basic stimuli ( $S 1-S 1=250 \mathrm{~ms}$ ). S2 is first premature beat given $154 \mathrm{~ms}$ after S1. S3 second premature beat given $116 \mathrm{~ms}$ after S2. Arrows indicate direction of activation. Locations c electrograms around ring are indicated in S1 map by large encircled numbers. Activation maps correspon to S1, S2, S3, and first reentrant beat. Numbers indicate local actiontion times in milliseconds. Isochrone are drawn at $10 \mathrm{~ms}$ intervals. Double bars indicate conduction block. See text for further description. 
intrinsic deflection. During transverse conduction (electrograms 7 and 8) the extracellular waveforms were of low amplitude. At the site of collision (electrogram 6) a monophasic positive deflection was recorded. No areas of local conduction delay or fragmentation of electrograms were observed at any site around the ring. All of these characteristics point to the fact that in these 17 experiments the ring consisted of uniform anisotropic myocardium. In three of 20 experiments during pacing impulse conduction around the obstacle was nonuniform because of the presence of an area of local conduction delay in some part of the ring. These three experiments were excluded from further analysis.

\section{Initiation of Ventricular Tachycardia}

VT was initiated by premature beats at 43 sites in 14 hearts. At three sites VT was induced by a single premature beat. At 12 other sites two premature beats were required; in the remaining 28 sites VT could be inducedonly by three premature beats. In figure 4.3 an example of the occurrence of unidirectional block and subsequent induction of sustained reentrant VT is given. Ten selected electrograms around the obstacle and for activation maps during induction of VT by two prema-ture are shown. The position of the electrograms around the ring is shown in the upper left map. During S1 (basic pacing at $250 \mathrm{~ms}$ intervals) the impulse propagated from the site of stimulation at the posterior base of the left ventricle around both sides of the ring to collide after $67 \mathrm{~ms}$ in the corridor between the LAD and the obstacle (electrogram 5). During S2 (first premature beat given $154 \mathrm{~ms}$ after S1) the wave fronts propagated at slower velocity resulting in collision after $79 \mathrm{~ms}$. During S3 (second premature beat given $116 \mathrm{~ms}$ after S2) the impulse was blocked at $\mathrm{t}=30 \mathrm{~ms}$ in the base of the left ventricle. The clockwise wave front continued to propagate at decreased speed because of the high degree of prematurity of the impulse and passed the site where collision occurred during regular pacing at $\mathrm{t}=113 \mathrm{~ms}$. Because now the clockwise wave front did not encounter an opposed wave front, it conti-nued its course and arrived at the site of unidirectional block $130 \mathrm{~ms}$ after block occurred. This delay of $130 \mathrm{~ms}$ was sufficient for the cells proximal to the line of block to recover their excitability and the impulse continued to propagate in a clockwise reentrant pathway. the resulting sustained VT had a regular cycle length of $128 \mathrm{~ms}$.

\section{Characterization of Ventricular Tachycardia}

The cycle lengths of the reentrant VTs in different experiments ranged from 128 to 198 $\mathrm{ms}$ (mean $161 \pm 19 \mathrm{~ms}, \mathrm{n}=43$ ). In Figure 4.4 two examples of sustained reentrant VT with cycle lengths of 136 and $195 \mathrm{~ms}$ are shown. Differences in VT cycle lengths were caused mainly by differences in the length of the segment of slow transverse conduction in the corridor between the LAD and the obstacle. Fast VTs showed a relatively short segment of slow conduction (left panel) whereas slow VTs exhibited a longer segment of slow transverse conduction (right panel). VT occurred 22 times clockwise and 21 times counterclockwise. In experiments in which more than one VT was induced the cycle lengths of different VTs were constant $(6 \mathrm{~ms})$ and indepen-dent of the site of induction or direction of propagation (clockwise or counter-clockwise). Once initiated spontaneous termination of VT was never observed.

Meaurements of the refractory period period during nine sustained VTs with a mean cycle length of $174 \pm 12 \mathrm{~ms}$ around the ring showed that the refractory period was 131 $\pm 18 \mathrm{~ms}$ leaving an excitable gap during sustained VT of $43 \pm 16 \mathrm{~ms}$. 


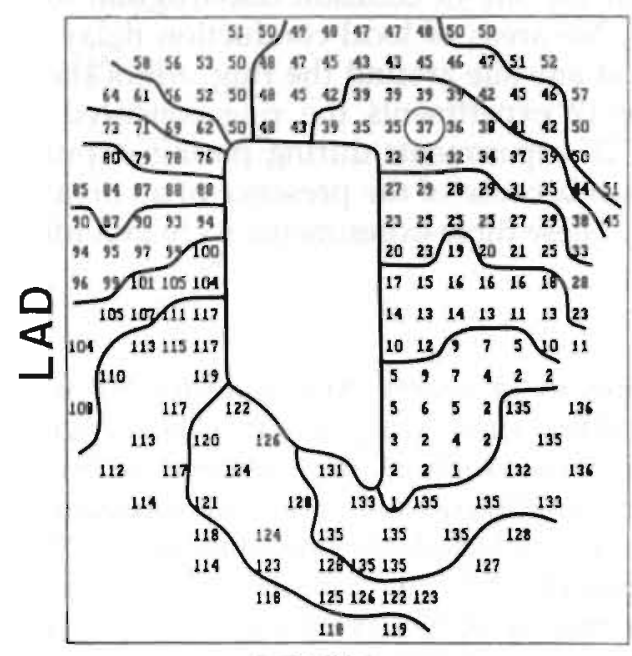

APEX

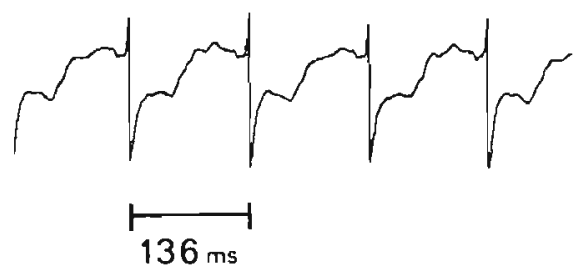

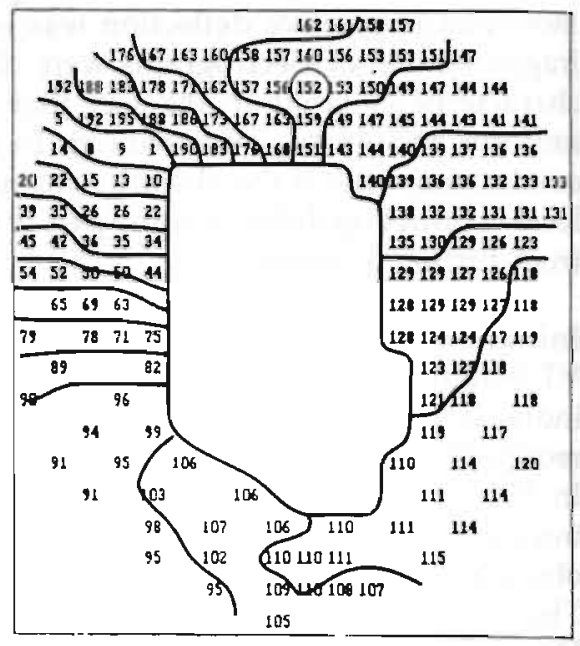

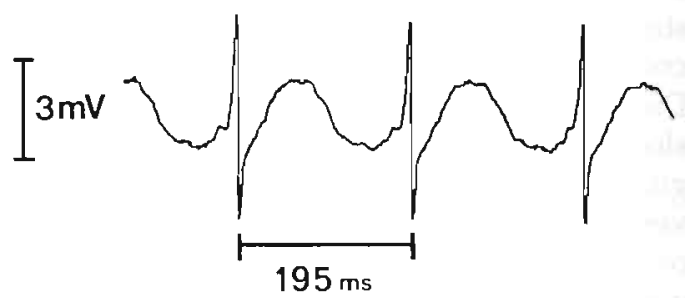

Figure 4.4: Activation maps and corresponding electrograms (location indicated by encircled activation time) during counterclockwise tachycardia with cycle lengths of 136 and $195 \mathrm{~ms}$ in two different experiments. Notice the difference in length of slow transverse conduction in corridor between LAD and obstacle in the two tachycardias. Numbers indicate local activation times in milliseconds. Isochrones are drawn at $10 \mathrm{~ms}$ intervals.

During sustained reentrant excitation conduction velocities differed depending on the angle between the rotating activation wave and the epicardial fiber direction. In Table 4.1 the conduction velocities are given in eight segments around the ring together with the angle between epicardial fiber orientation and direction of impulse propagation. In the corridor between the LAD and the obstacle where the excitation wave travelled perpendicular to fiber orientation (segment 2) mean conduction velocity was $25 \pm 5$ $\mathrm{cm} / \mathrm{s}$ (range 18-32 cm/s). At the base of the left ventricle where the impulse travelled parallel to the fiber orientation (segment 5) mean conduction velocity was $68 \pm 4 \mathrm{~cm} / \mathrm{s}$ (range $53-72 \mathrm{~cm} / \mathrm{s}$ ).

In four experiments impalements with standard glass microelectrodes filled with $3 \mathrm{M}$ $\mathrm{KCl}$ were used to map electrical activation at different depths in the epicardial layer during reentrant VT. Impalements from superficial layers of surviving epicardium during reentrant VT revealed no differences in activation times. 
Table 4.1.:

Correlation between Fiber Orientation, Direction of Propagation, and Conduction Velocity.

\begin{tabular}{ccccc}
\hline $\begin{array}{c}\text { Segment } \\
\text { in Ring }\end{array}$ & $\begin{array}{c}\text { Fiber } \\
\text { Orientation }\end{array}$ & $\begin{array}{c}\text { Direction of } \\
\text { Propagation }\end{array}$ & $\begin{array}{c}\text { Angle between } \\
\text { Direction of } \\
\text { Propagation and } \\
\text { Fiber Orientation } \\
\text { (degrees) }\end{array}$ & $\begin{array}{c}\text { Conduction } \\
\text { Velocity }\end{array}$ \\
\hline 1 & $8 \pm 3$ & $82 \pm 4$ & $74 \pm 3$ & (cm/s) \\
\hline 2 & $4 \pm 2$ & $95 \pm 3$ & $91 \pm 3$ & $25 \pm 5$ \\
3 & $2 \pm 2$ & $127 \pm 7$ & $125 \pm 5$ & $32 \pm 8$ \\
4 & $6 \pm 3$ & $8 \pm 8$ & $2 \pm 6$ & $55 \pm 6$ \\
5 & $22 \pm 5$ & $23 \pm 4$ & $1 \pm 4$ & $68 \pm 4$ \\
6 & $45 \pm 4$ & $47 \pm 6$ & $2 \pm 5$ & $61 \pm 5$ \\
7 & $65 \pm 6$ & $69 \pm 6$ & $4 \pm 6$ & $53 \pm 7$ \\
8 & $74 \pm 6$ & $118 \pm 9$ & $48 \pm 7$ & $36 \pm 6$ \\
\hline
\end{tabular}

Anatomic correspondence of segments in ring is given in figure 4.1. All values are given as mean \pm standard deviation (See "Methods").

Characterization of the Area of Unidirectional Block during Initiation of Ventricular Tachycardia

In all 43 cases in which reentrant excitation was initiated by premature stimulation, the localization of the area of unidirectional block could be identified. At the sites of block the surviving rim of epicardium was not thinner than the average thickness of the surviving epicardial layer nor were they associated with the presence of major coronary vessels on the surface of the heart. In Figure 4.5 the localization of the 43 lines of unidirectional block in the ring are shown schematically. In 41 cases unidirectional block occurred at the base of the left ventricle where the impulse propagated parallel to the fiber direction. In these cases the average angle between fiber orientation and direction of propagation at the site of block was $2 \pm 4$ and conduction velocity during VT was $65 \pm 4 \mathrm{~cm} / \mathrm{s}$. Only in two cases was block found in the corridor between the LAD and the obstacle where propagation was transverse to the fiber axis. In these two cases the angle between fiber orientation and direction of propagation at the site of block was $88 \pm 4$ degrees and conduction velocity was $22 \pm 2 \mathrm{~cm} / \mathrm{s}$.

In Figure 4.3 an example of longitudinal block is given. The electrograms across the line of block were of high amplitude (electrograms 8 and 9) and localconduction velocity was $71 \mathrm{~cm} / \mathrm{s}$. In figure 4.6 one of the two cases of transverse conduction block is shown. On the left the activation maps during regular pacing (S1) and a single prema- 


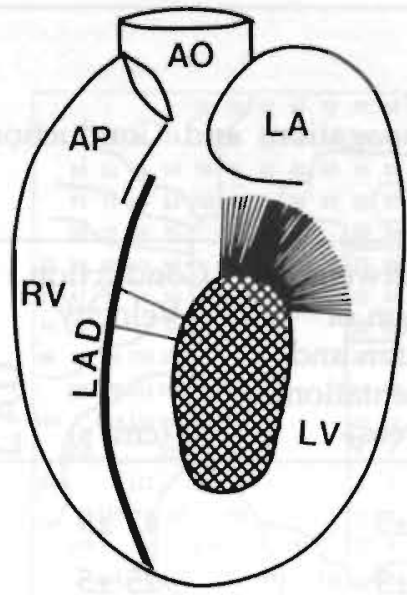

Figure 4.5: Schematic drawing of localization of all 43 lines of unidirectional block that initiated reentrant tachycardia. All except two were located in base and upper part of free wall of left ventricle where conduc-tion was parallel to fiber orientation. $L A D=$ Left Anterior Descending artery; $A O=$ aorta; $A P=$ pulmo-nary artery; $L A=$ left atrium; $R V=$ right ventricle; $L V=$ left ventricle.

ture beat (S2;coupling interval $143 \mathrm{~ms}$ ) are shown. On the right 6 selected electrograms around the ring are given. During $\mathrm{S} 1$ the impulse propagated along both sides of the obstacle to collide in the area of electrode 4 at the lower left of the ring $133 \mathrm{~ms}$ after the stimulus was given. The premature impulse (S2) blocked in a counterclockwise direction at the area of transverse conduction at electrode 5. During regular pacing (upper panel) conduction velocity at the area of electrodes 5 and 6 was $20 \mathrm{~cm} / \mathrm{s}$ and the electrograms were of relatively low amplitude. The clockwise premature wavefront propagated at a reduced conduction velocity and a reentrant VT with a cycle length of 160 ms was started.

\section{Local Dispersion in Refractoriness and Inducibility of Ventricular Tachycardia}

To determine whther local dispersion in refractory period played a role in the occurrence of unidirectional block the refractory periods proximal and distal to the line of block were compared. In 22 of 43 cases unidirectional block occurred while the impulse was travelling from an area with a longer into an area with a shorter refractory period $(-8 \pm 3 \mathrm{~ms})$. In nine of 43 cases was travelling through an area with a less than $10 \mathrm{~ms}$ prolonged refractory period $(+4 \pm 2 \mathrm{~ms})$. In only 12 of 43 cases unidirectional block occurred when the local refractory period distal to the site of block was more than $10 \mathrm{~ms}$ longer than the refractory period proximal to the block $(+19 \pm 18 \mathrm{~ms})$. In the two cases in which unidirectional block occurred transverse to the fiber orientation the impulse propagated towards an area with a markedly prolonged refractory period. In the example in Figure 4.6 the refractory period at site 5 was $125 \mathrm{~ms}$ while the area between electrodes 5 and 4 had a refractory period of $158 \mathrm{~ms}$. In the other case the local difference in refractory period was $+28 \mathrm{~ms}$.

During repeated induction of both clockwise and counterclockwise reentrant VTs in five experiments unidirectional block occurred at the same area regardless of the direction of propagation. This is illustrated in Figure 4.7 in which activation maps during induction of both counterclockwise and clockwise VTs are shown. When the heart was paced at the anterior base of the left ventricle (upper panels) the third premature stimulus (\$4;coupling interval $132 \mathrm{~ms}$ ) blocked clockwise between points 1 and 3 . Conduction velocity between electrodes 1 and 3 during regular pacing was $72 \mathrm{~cm} / \mathrm{s}$. The refractory period at site 1 proximal to the line of block was $138 \mathrm{~ms}$ whereas the 

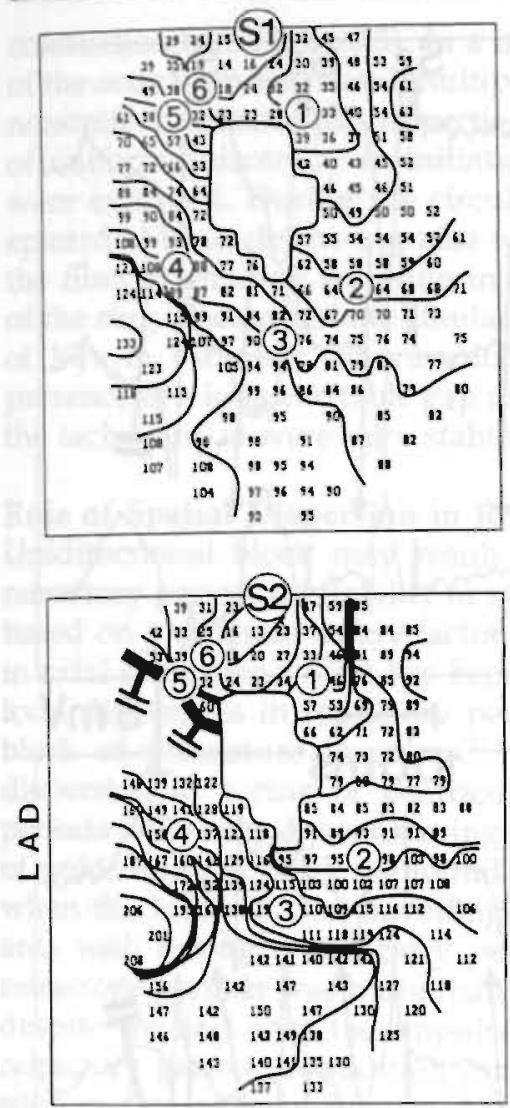

APEX

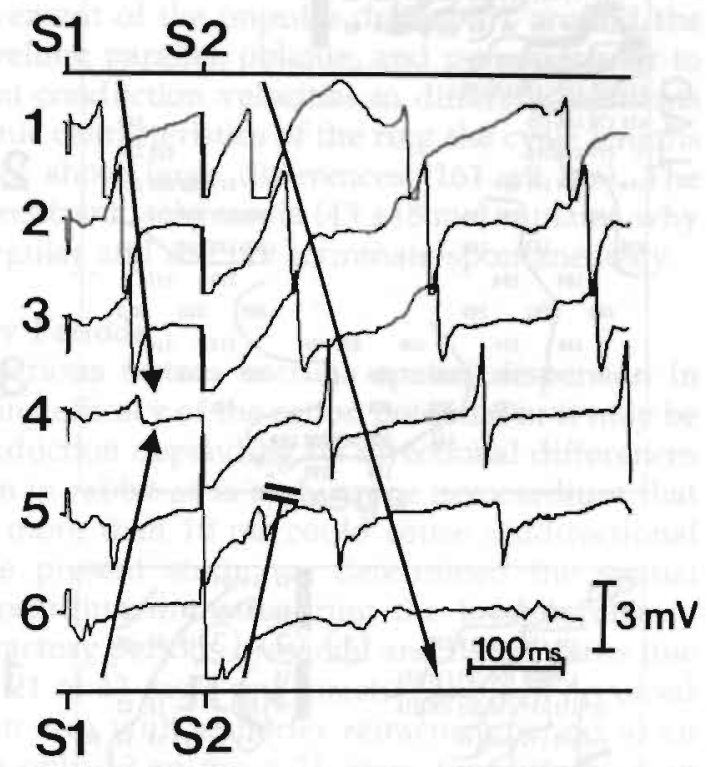

Figure 4.6: Two activation maps and six selected electrograms around obstacle during regular pacing (S1) and application of a single early premature beat (S2, coupling interval $143 \mathrm{~ms}$ ) that started sustained VT. In this exceptional case, unidirectional block occurred during slow propagation transverse of fiber axis. Numbers indicate local activation times in milliseconds. Isochrones are drawn at $10 \mathrm{~ms}$ intervals. Double bars indicate conduction block. LAD $=$ Left Anterior Descending artery. See text for further description.

refractory period at site 3 distal to the line of block was $146 \mathrm{~ms}$. However, when the heart was paced at a posterior site (lower panels) the third premature stimulus (S4; coupling interval $136 \mathrm{~ms}$ ) was blocked in the same area. Now block occurred when the impulse was propagating in a counterclockwise direction travelling from an area with a longer $(146 \mathrm{~ms})$ into and area with a shorter refractory period $(138 \mathrm{~ms})$. Thus, in this case block occurred regardless of the local differences in refractory period.

\section{DISCUSSION}

We developed an experimental model consisting of a ring of anisotropic ventricular epicardium in Langendorff perfused rabbit hearts. In the majority of experiments 

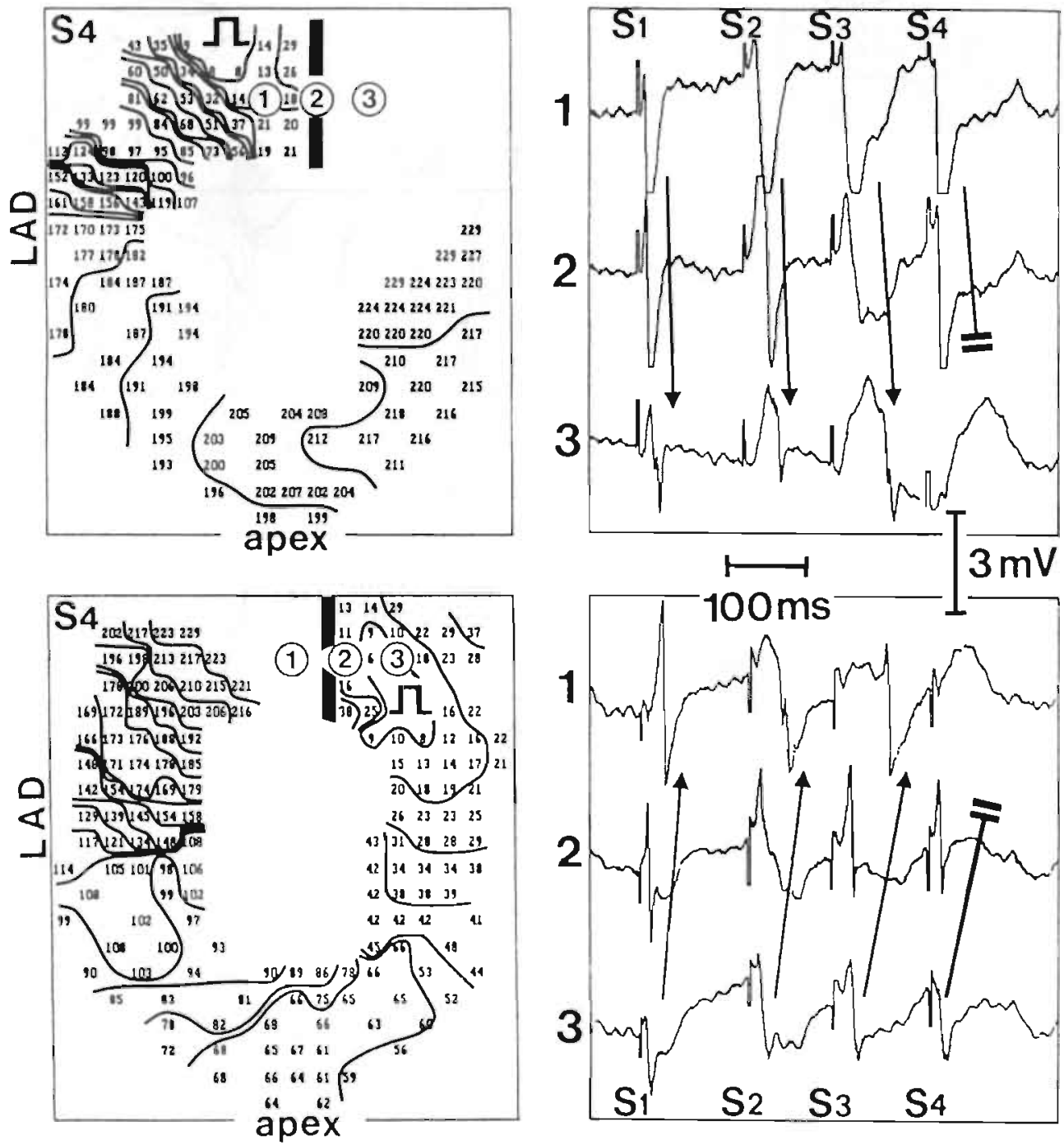

Figure 4.7: Activation maps and three selected electrograms during initiation of undirectional block when pacing pacing at anterior (top) and posterior (bottom) bases of left ventricle. Unidirectional block occurred at sume area (heavy line) regardless of whether premature impulse propagated from left to right or from right to left. Numbers indicate local activation times in milliseconds. Isochrones are drawn at $10 \mathrm{~ms}$ intercals. Position of electrograms is indicated by large encircled numbers. Double bars indicate conduction block. $L A D=$ Left Anterior Descending artery. See text for further description.

continuous left ventricular epicardial mapping showed smooth propagation of the wave front and nonfragmented extracellular potentials all around the circuit, suggesting uniform anisotropic properties. ${ }^{9.10}$ During regular pacing conduction velocity parallel to the fiber orientation was $62 \pm 6 \mathrm{~cm} / \mathrm{s}$ compared to $27 \pm 4 \mathrm{~cm} / \mathrm{s}$ during transverse 
conduction (ratio $2.7 \pm 0.3$ ). In a minority of experiments (three of 20 ) irregular spread of the activation wave and multiple component electrograms were recorded suggesting nonuniform anisotropic properties. Because in this study we wanted to focus on the role of uniform anisotropy in initiation of reentrant tachycardia these three experiments were excluded. During the circular movement of the impulse during VT around the epicardial obstacle the impulse was travelling parallel, oblique, and perpendicular to the fiber orientation resulting in different conduction velocities in different segments of the ring. Because of the similar anatomic characteristics of the ring the cycle lengths of VTs in different experiments did not show large differences (161 $\pm 9 \mathrm{~ms})$. The presence of a long excitable gap during reentrant tachycardia ( $43 \pm 18 \mathrm{~ms}$ ) explains why the tachycardias were very stable and regular and did not terminate spontaneously.

\section{Role of Spatial Dispersion in Refractory Periods}

Unidirectional block may result from various factors such as spatial dispersion in refractory period, excitability or stimulating efficacy of the action potential or it may be based on a different safety factor for conduction depending on directional differences in axial resistance..$^{311-13}$ It has been shown in rabbit atria and canine myocardium that local differences in refractory period of more than $10 \mathrm{~ms}$ could cause unidirectional block of premature impulses. ${ }^{11,12}$ In the present study we determined the spatial dispersion in a ring of ventricular myocardium by measuring the local refractory periods at 16 sites. By comparing the refractory periods proximal and distal to the line of unidirectional block we found that in 21 of 43 cases uni-directional block occurred when the impulse was travelling from an area with a shorter refractory period to an area with a longer refractory period. In only 12 of these 21 cases the difference in refractory periods was more than $10 \mathrm{~ms}$. In 22 of 43 cases unidirctional block occurred despite the fact that the impulse was propagating towards an area with a shorter refractory period. In addition during repeated induction of VT from different pacing sites in five experiments unidirectional block occurred at the same area while the premature impulse was propagating in opposite directions. These observations indicate that in the majority of cases spatial dispersion in refractory periods could not account for local conduction block of the premature impulses. On the other hand because in most cases VT was initiated by two or three premature beats one might argue that spatial differences in refractory periods of single or multiple premature beats might be different compared with the distribution of refractory periods during basic rhythm.

\section{Role of Anisotropy in Initiation of Ventricular Tachycardia}

In the vast majority of cases unidirectional block occurred in a segment of the ring where the impulse was propagating parallel to the fiber orientation; only two exceptions were found in which the premature impulses blocked during propagation transverse to the fiber orientation. In these two cases a large difference in refractory period was found at both sides of the arc of block. Our results are in agreement with the rsults of Spach et al. ${ }^{3}$ and Tsuboi et al. ${ }^{11}$ They suggested that in anisotropic tissue the safety factor for longitudinal conduction is lower compared with transverse conduction leading to preferential longitudinal conduction block when the amount of excitatory current generated by the myocardial fibers is depressed by either premature impulses or increased extracellular potassium. In isolated canine atria Spach et al. ${ }^{13}$ showed that anisotropy may interact with inhomogeneities in repolari-zation to create favorable circumstances for reentry. In about half of our cases in which unidirectional 
block occurred while the impulse was propagating into an area with a slightly longer refractory period we cannot exclude that local dispersion in refractoriness interacted with the anisotropic conduction properties to produce local conduction block. However, in the other half of our experiments unidirectional block occurred while the impulse was actually propagating nto an area with a shorter refractory period and spatial differences in refractory period could be excluded from being responsible for local conduction block. In these cases it is conceivable that a lower safety factor for conduction during longitudinal propagation was the underlying mechanism for unidirectional block and consequent initiation of reentry.

\section{CONDENSED ABSTRACT}

The relative influence of tissue anisotropy and dispersion of refractoriness on initiation of reentrant ventricular tachycardia were studied in an experimental model consisting of a ring of uniform anisotropic ventricular epicardium in a Langendorff perfused rabbit heart. One to three premature stimuli were applied at 16 different pacing sites in each of 17 hearts. In 43 cases VT was initiated by unidirectional conduction block of a premature wave front. Correlation of the conduction velocity and the epicardial fiber orientation at the segment of unidirectional block showed that block predominantly occurred while the impulse propagated parallel to the fiber direction. In $72 \%$ of cases the refractory period distal to the site of block was shorter than proximal to the site of block. From these results we conclude that reentrant tachycardia can be intiated by premature stimuli due to preferential unidirectional conduction block parallel to the epicardial fiber orientation. 


\section{REFERENCES}

1. Mines GR: On circulating excitations in heart muscles and their possible relation to tachycardia and fibrillation. Trans Soc Can 1914;5:43-53

2. Clerc L: Directional differences of impulse spread in trabecular muscle from mammalian heart. J Physiol (London) 1976;255:335-346

3. Spach M, Miller W, Geselowitz D, Barr R, Kootsey J, Johnson E: The discontinuous nature of propagation in normal canine cardiac muscle: Evidence for recurrent discontinuities of intracellular resistance that affect the membrane currents. Circ Res 1981;48:39-54

4. Brugada J, Boersma L, Kirchhof C, Brugada P, Havenith M, Wellens H, Allessie M: Double-wave reentry as mechanism of ventricular tachycardia acceleration. Circulation 1990;81:1633-1643

5. Allessie M, Schalij M, Kirchhof C, Boersma L, Huyberts M, Hollen J: Experimental electrophysiology and arrhythmogenicity: Anisotropy and ventricualr tachyardia. Eur Heart J 1989;10:2-8

6. Allessie M, Hoeks A, Schmitz F, Reneman R: On-line mapping system for the visualization of the electrical activation of the heart. Int J Card Imaging 1988;2:59-63

7. Nachlas M, Schnitka T: Macroscopical identification of early myocardial infarcts by alteration in dehydrogenase activity. Am J Pathol 1963;4:379-405

8. Gordon H, Sweets H: Simple method for the silver impregnation of reticulum. Am J Pathol $1936 ; 12: 545-552$

9. Spach M, Miller W, Miller-Jones E, Warren R, Barr R: Extracellular potentials related to intracellular action potentials during impulse conduction in anisotropic canine cardiac muscle. Circ Res 1979;45:188-204

10. Spach M, Dolber P: Relating extracellular potentials and their derivatives to anisotropicpropagati on at a microscopic level in human cardiac muscle: Evidence for electrical uncoupling of side-toside fiber connections with increasing age. Circ Res 1986;58:356-371

11. Allessie M, Bonke F, Schopman F: Circus movement in rabbit atrial muscle as a mechanism of tachycardia: II. The role of nonuniform recovery of excitability in the occurrence of unidirectional block as studied with multiple microelectrodes. Circ Res 1976;39:168-177

12. Gough W, Mehra R, Restivo M, Zeiler R, El-Sherif N: Reentrant ventricular arrhythmias in the late myocardial infarction period in the dog: 13. Correlation of activation and refractory periods. Circ Res 1985; $57: 432-442$

13. Spach M, Dulber P, Heidlage J; Interaction of inhomogeneities of repolarazation with anisotropic propagation in dog atria: A mechanism for both preventing and initiating reentry. Circ Res $1989 ; 65: 1612-1631$

14. Tsuboi N, Kodama I, Toyama J, Yamada K: Anisotropic conduction properties of canine ventricular muscles: Influence of high extracellular $\mathrm{K}^{+}$concentration and stimulation frequency. Jpn Circ J 1985;49:487-498 

CHAPTER 5

THE EFFECTS OF $\mathrm{K}^{+}$ON ANISOTROPIC CONDUCTION IN SHEETS OF PERFUSED RABBIT VENTRICULAR MYOCARDIUM.

Lucas Boersma Josep Brugada Martin Jan Schalij Charles Kirchhof Maurits Allessie

J of Cardiovasc Electrophysiol 1991;2:492-502 


\section{INTRODUCTION}

During the last years, several studies have been performed to investigate the influence of anisotropic tissue structure on impulse propagation, local conduction block and the initiation of cardiac arrhythmias. Sano et al. ${ }^{1}$ were among the first to show that anisotropy of cardiac muscle caused directional differences in conduction velocity with fast conduction parallel and slow conduction perpendicular to the fiber orientation. In a later study, Clerc $^{2}$ showed that the axial resistance transverse to the fiber orientation was about 10 times higher than in longitudinal direction. Since the conduction velocity is inversely related to the square root of the axial resistance ${ }^{3}$, this would result in a ratio of about 3 between longitudinal and transverse conduction velocity. Spach et al. ${ }^{4,5,6}$ showed that because of the discontinuous nature of propagation in anisotropic myocardium, a high conduction velocity is not necessarily associated with a high safety factor for conduction. Premature impulses propagating in longitudinal direction were found to have a lower $\mathrm{dV} / \mathrm{dt}$ max, resulting in conduction block while transverse conduction still proceeded. Such a lower margin of safety for conduction parallel to the fiber orientation was explained by a greater loss of excitatory current, due to the lower resistance along the longitudinal fiber axis. Later studies performed by Tsuboi et al. , Kadish et al. ${ }^{8}$ and Osaka et al. ${ }^{9}$ confirmed this "current sink" hypothesis. However, in other studies ${ }^{10,11,12}$ opposite results were found, indicating that depression of the fast sodium channels resulted in preferential conduction block transverse to the fiber orientation.

In the light of these discrepancies found in the literature, we studied the effects of increasing extracellular $\mathrm{K}^{+}$on anisotropic conduction in a perfused sheet of rabbit epicardium by high resolution mapping. Low stimulating frequencies were used to exclude the possibility that differences in action potential configuration or local refractory periods might affect conduction velocity. Detailed analysis of the the epicardial spread of activation during stepwise increase of $\mathrm{K}^{+}$did not reveal a consistent change in the ratio of anisotropic conduction. Also, no experimental evidence was found for preferential conduction block either in longitudinal or in transverse direction.

\section{METHODS}

\section{Preparation}

In this study, in 10 Langendorff-perfused rabbit hearts a thin layer of left ventricular subepicardium was created by the cryoprocedure. The perfusion system and the cryoprocedure have been discussed in chapter 2 . After the experiments, sections were cut parallel the epicardial surface and the epicardial fiber orientation was determined by silver impregnation of the reticulum. ${ }^{13}$

\section{Recording and stimulation}

A rectangular mapping electrode, containing 240 silver electrodes (diameter $0.3 \mathrm{~mm}$ ) arranged in a regular array of $16 \times 15$ recording points, was used for high resolution mapping of an area of $15 \times 14 \mathrm{~mm}$ of the epicardium of the left ventricle. Unipolar electrograms were recorded, using the cannula in the aorta as indifferent electrode. The on-line mapping system used to record and analyze the data has been described in chapter 3. 
The ventricle was paced at a slow constant rate (interval $2000 \mathrm{~ms}$ ) through a pair of electrodes in the center of the mapping electrode. A constant current stimulator was used to deliver square pulses of $2 \mathrm{~ms}$ duration with an amplitude of twice diastolic threshold. Starting at $6.0 \mathrm{mM}$, the extracellular concentration of potassium was increased in steps of 1.0-2.0 mM. Samples of the perfusate were collected from the outflow of the heart and the potassium concentration was determined with a flamephotometer (model 243, Fisher Medical Division Instrumentation Laboratory, Lexington, MA, USA).

\section{Data Analysis}

After each experiment, the 240 electrograms were played back from videotape for detailed analysis of the epicardial spread of activation at different potassium concentrations. An algorithm was used to detect the steepest negative deflection in each electrogram as the moment of local activation. Activation maps were plotted and isochrones were drawn at $10 \mathrm{~ms}$ intervals. If necessary, the maps were edited by manual correction of the activation marks in the electrograms. The longitudinal electrical axis of propagation was determined from the ellipsoidal shape of the isochrones. Conduction velocities along the two axes of the ellipse were calculated from the distance separating the isochrones and the difference in activation time (starting at $3 \mathrm{~mm}$ from the site of stimulation). The mean of the two values obtained for each axis was taken as $\theta_{\mathrm{L}}$ and $\theta_{\mathrm{T}}$ to calculate the ratio of anisotropy. A local conduction delay of more than $40 \mathrm{~ms}$ between two neighbouring electrodes (conduction velocity $<2.5 \mathrm{~cm} / \mathrm{s}$ ) was interpreted as local conduction block. From previous studies on anisotropic conduction, it is known that conduction can sometimes proceed very slowly (conduction velocity 5 to $10 \mathrm{~cm} / \mathrm{s}$ ) across a line of apparent conduction block. ${ }^{14}$ Therefore, as an additional criterion for the presence of conduction block, a change in direction of activation distal to the line of conduction block had to be present.

\section{Statistical Analysis}

Linear regression analysis and the paired Student T-test were used to compare conduction velocities at different potassium concentrations. Average values are given as the mean \pm the standard deviation. Differences were considered to be statistically significant at $\mathrm{p}$-values of $<0.05$.

\section{RESULTS}

In figure 5.1, an activation map of the epicardial spread of activation during slow pacing (interval $2000 \mathrm{~ms}$ ) at a potassium concentration of $6.0 \mathrm{mM}$ is given, together with the histological determination of the epicardial fiber orientation. From the site of stimulation in the center of the mapping electrode, the impulse propagated smoothly towards all directions. The anisotropic tissue properties are expressed in the activation map by a clear elliptical shape of the isochrones with the fast electrical axis oriented slightly oblique from left to right. The histological determination of the epicardial fiber direction in several areas under the mapping electrode showed that the long axis of the ellipsoid isochrones was oriented parallel to the fiber axis and that the broad slowly conducting wavefronts were propagating transverse to the fiber direction. 

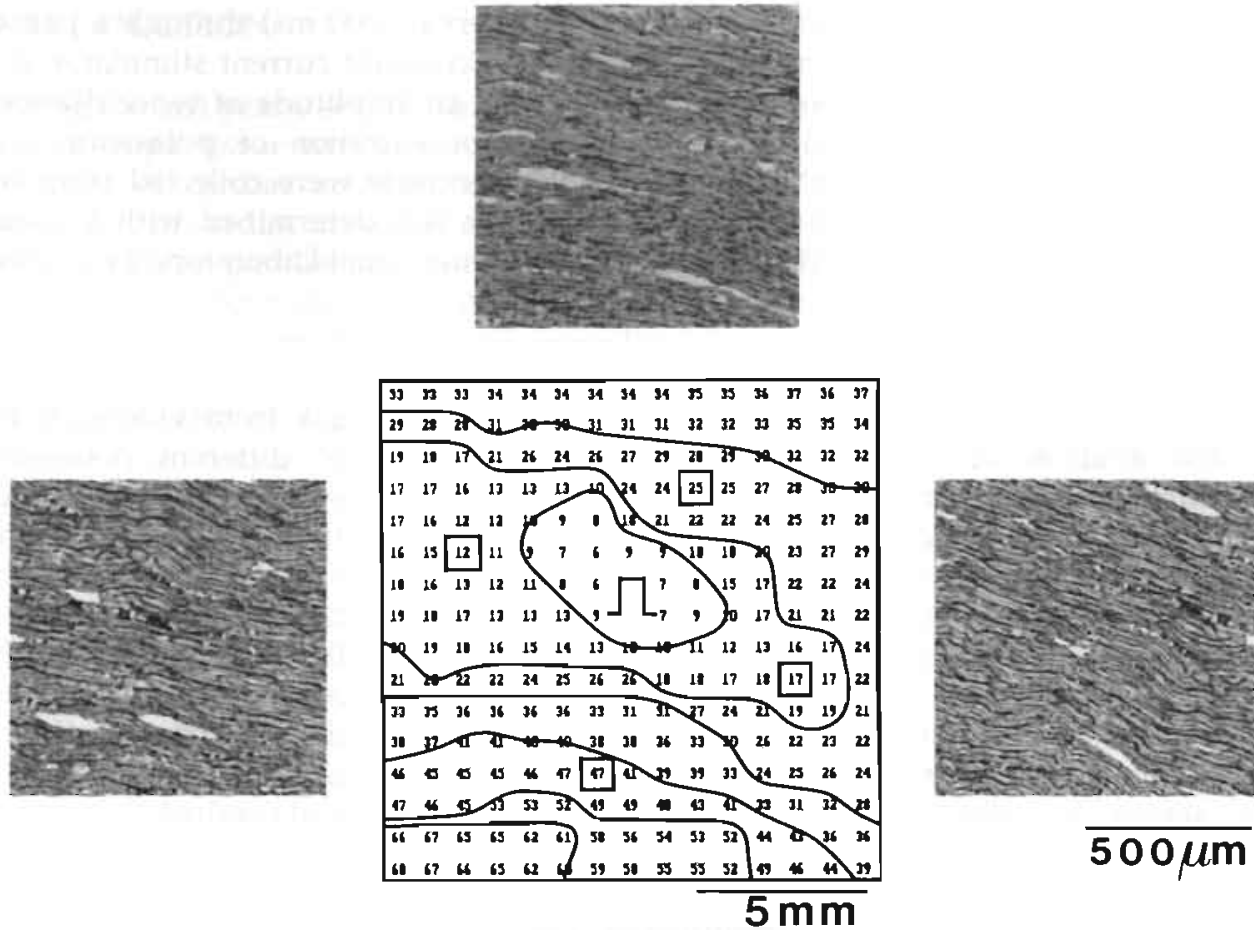

$\overline{500 \mu \mathrm{m}}$

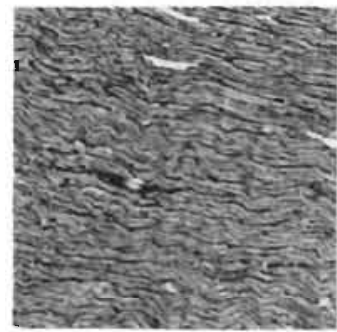

Figure 5.1: Activation map of the epicardial spread of activation during pacing in the center of the electrode array at $0.5 \mathrm{~Hz}$ at an extracellular $\mathrm{K}^{+}$concentration of $6.0 \mathrm{mM}$. The actioation times at all recording points are given in milliseconds and isochrones are drawn at $10 \mathrm{~ms}$ intervals. The four histological sections showing the local fiber orientation were taken from the areas indicated by the open squares in the map. The electrical axis of fast propagation was oriented parallel to the long axis of the epicardial fibers.

The effects of increased extracellular potassium on epicardial activation

To study the effects of progressive depression of the active generator properties of the cell membrane on anisotropic conduction, the potassium concentration in the perfusion fluid was increased in steps of 1.0-2.0 mM. At each concentration an activation map of the epicardial spread of activation was reconstructed. An example is shown in figure 5.2. At $6.0 \mathrm{mM}$ a uniform elliptical spread of activation was present. From the site of stimulation two longitudinal wavefronts propagated in opposite direction with a conduction velocity of 61 and $60 \mathrm{~cm} / \mathrm{s}$ respectively. The two broad transverse 

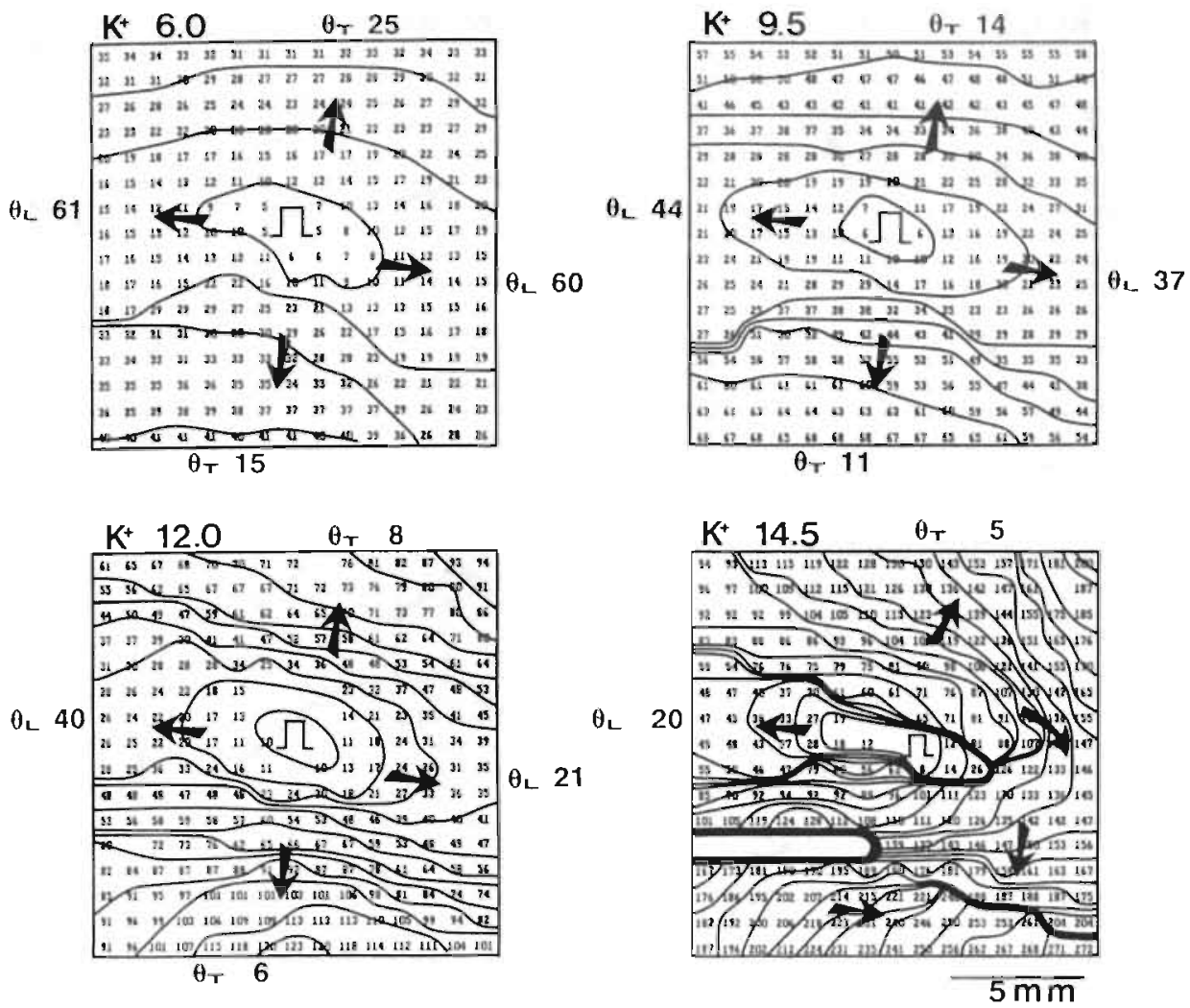

Figure 5.2: Activation maps during slow regular pacing $(0.5 \mathrm{~Hz})$ at $6.0,9.5,12.0$, and $14.5 \mathrm{mM}$ of $\mathrm{K}^{*}$. Local activation times are given in milliseconds and isochrones are drawn at $10 \mathrm{~ms}$ intervals. The long electrical axis of the propagating wave is oriented from left to right. Arrous indicate the direction of the major longitudinal and transverse wavefronts and the respective conduction velocities $\left(\theta_{L}\right.$ and $\left.\theta_{T}\right)$ are given alongside the map. Thick black lines at $14.5 \mathrm{mM}$ of $\mathrm{K}^{+}$represent local functional conduction block.

wavefronts propagated with a velocity of 25 (up) and $15 \mathrm{~cm} / \mathrm{s}$ (down). When the potassium concentration was raised to $9.5 \mathrm{mM}$ the number of isochrones increased in all directions. Longitudinal conduction velocity was decreased to 44 and $37 \mathrm{~cm} / \mathrm{s}$ and transverse conduction to 14 and $11 \mathrm{~cm} / \mathrm{s}$. The elliptical shape of the isochrones was maintained indicating that conduction was still anisotropic. At $12.0 \mathrm{mM}$ conduction was further depressed to 40 and $21 \mathrm{~cm} / \mathrm{s}$ longitudinally and 8 and $6 \mathrm{~cm} / \mathrm{s}$ transverse to the fiber orientation. At a $\mathrm{K}^{+}$concentration of $14.5 \mathrm{mM}$, conduction became very slow and local arcs of conduction block appeared. However propagation to the left and in upward direction continued at a more or less constant speed with an effective $\theta_{\llcorner}$of 20 $\mathrm{cm} / \mathrm{s}$ and $\mathrm{a} \theta_{\mathrm{T}}$ of $5 \mathrm{~cm} / \mathrm{s}$. In both transverse directions the latency between the moment of the stimulus and the actual start of propagation was largely increased to about 60 ms. Lines of conduction block with interelectrode time delays of 40-100 ms were found both in transverse and longitudinal direction. The areas distal to the arcs of block were 

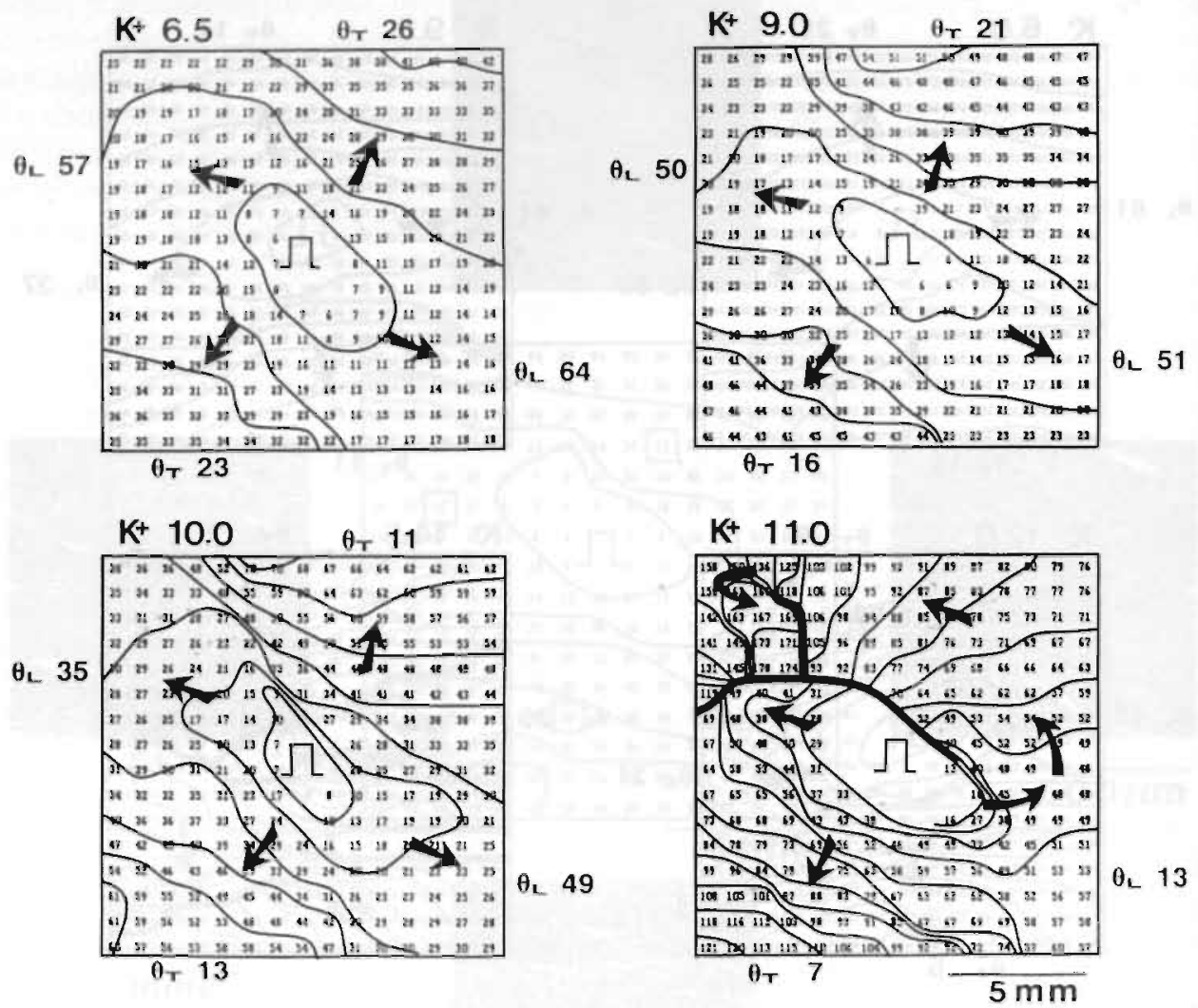

Figure 5.3: Activation maps during slow regular pacing $(0.5 \mathrm{~Hz})$ at $6.5,9.0,10.0$, and $11.0 \mathrm{mM}$ of $\mathrm{K}^{+}$. The longitudinal axis is oriented oblique to the mapping electrode. Arrows indicate the direction of the major longitudinal and transverse wavefronts together with their respective $\theta_{\mathrm{L}}$ and $\theta_{T}$. The thick black lines in the lower right panel represent local conduction block.

activated in a different direction by wavefronts turning around the line of block. In the lower left part of the mapping electrode a long arc of block enveloped an area that could not be activated by the propagating wave fronts. At a slightly higher potassium concentration $(>15.0 \mathrm{mM})$ the ventricle became completely inexcitable. In this experiment the ratio of anisotropy increased at higher extracellular $\mathrm{K}^{+}$because transverse conduction was more depressed then conduction parallel to the fiber orientation. At a potassium concentration of $6.0 \mathrm{mM}$ the ratio was 3.0 and at progressively higher extracellular $\mathrm{K}^{+}$of $9.5,12.0$ and $14.5 \mathrm{mM}$ increased to $3.2,4.4$ and 4.0 respectively.

In figure 5.3 another experiment is shown. At $6.5 \mathrm{mM}$ of $\mathrm{K}^{+}$again a regular elliptical spread of activation was present with fast longitudinal conduction of 57 and $64 \mathrm{~cm} / \mathrm{s}$ and slow transverse conduction of 26 and $23 \mathrm{~cm} / \mathrm{s}$. In this case the long electrical axis was oriented oblique in relation to the electrode array. At 9.0 and $10.0 \mathrm{mM}$ of $\mathrm{K}^{+}$ conduction became progressively depressed but the elliptical shape of the isochrones 
Table 5.1:

Effects of high extracellular $\mathrm{K}^{+}$on longitudinal and tranverse conduction velocities.

\begin{tabular}{c|cccc|cccc}
\hline EXP & $\mathrm{K}^{+}$ & $\theta_{\mathrm{L}}$ & $\theta_{\mathrm{T}}$ & $\theta_{\mathrm{L}} / \theta_{\mathrm{T}}$ & $\mathrm{K}^{+}$ & $\theta_{\mathrm{L}}$ & $\theta_{\mathrm{T}}$ & $\theta_{\mathrm{L}} / \theta_{\mathrm{T}}$ \\
\hline 1 & 6.5 & 61 & 25 & 2.4 & 10.5 & 36 & 11 & 3.3 \\
2 & 6.0 & 64 & 32 & 2.0 & 12.5 & 39 & 6 & 6.5 \\
3 & 6.0 & 62 & 24 & 2.6 & 14.5 & 31 & 14 & 2.2 \\
4 & 6.5 & 49 & 22 & 2.2 & 12.0 & 25 & 13 & 1.9 \\
5 & 6.0 & 63 & 31 & 2.0 & 11.5 & 35 & 11 & 3.2 \\
6 & 6.5 & 68 & 28 & 2.4 & 12.0 & 34 & 21 & 1.6 \\
7 & 6.0 & 67 & 25 & 2.7 & 13.0 & 24 & 8 & 3.0 \\
8 & 6.0 & 61 & 20 & 3.1 & 13.0 & 22 & 6 & 3.7 \\
9 & 6.5 & 67 & 22 & 3.0 & 13.5 & 23 & 6 & 3.8 \\
10 & 6.0 & 51 & 16 & 3.2 & 12.0 & 23 & 10 & 2.3 \\
\hline AVG & 6.2 & 61 & 25 & 2.6 & $12.5^{*}$ & $29 *$ & $11^{*}$ & 3.2 \\
SD & \pm 0.2 & \pm 6 & \pm 5 & \pm 0.4 & \pm 1.1 & \pm 6 & \pm 4 & \pm 1.3 \\
\hline
\end{tabular}

$*=p<0.001$ (paired Student's $T$-test); $K^{*}=$ extracellular potassium concentration in millimol per liter; $\theta_{L}=$ longitudinal conduction velocity $(\mathrm{cm} / \mathrm{s}) ; \theta_{T}=$ transverse conduction velocity $(\mathrm{cm} / \mathrm{s}) ; \theta_{V} / \theta_{T}=$ ratio of anisotropy: $A V G=$ average $; S D=$ standard deviation.

was maintained. At $11.0 \mathrm{mM}$ of $\mathrm{K}^{+}$the pattern of excitation of the upper part of the preparation changed markedly because a long continuous arc of transverse and longitudinal conduction block developed. The area behind this line of block was now activated by a broad longitudinal wavefront turning counterclockwise around the right end of the line of transverse block. Total inexcitability of the ventricle occurred at a potassium concentration of more than $12.0 \mathrm{mM}$. In this example the changes in the ratio of $\theta_{\mathrm{L}}$ and $\theta_{\mathrm{T}}$ by potassium were not consistent. During an increase of $\mathrm{K}^{+}$to 9.0 and 10.0 $\mathrm{mM}$ the ratio increased from 2.5 to 2.7 and 3.5 respectively. However at a concentration of $11.0 \mathrm{mM}$ due to a strong depression of longitudinal conduction the ratio of anisotropy dropped sharply again to 1.9 .

Effects of increasing extracellular potassium on the ratio of anisotropic conduction In table 5.1 the individual values of $\theta_{\mathrm{L}}$ and $\theta_{\mathrm{T}}$ are given for all 10 experiments. The values during control $\left(\mathrm{K}^{+}\right.$6.0-6.5 $\left.\mathrm{mM}\right)$ are given together with the conduction velocities at the highest potassium concentration before local conduction block developed. During control the average value for $\theta_{\mathrm{L}}$ and $\theta_{\mathrm{T}}$ was $61 \pm 6.2$ and $25 \pm 4.6 \mathrm{~cm} / \mathrm{s}$ respectively with a ratio of anisotropy of $2.6 \pm 0.4$. At an average potassium concentration of $12.5 \pm 1.1$ $\mathrm{mM}$ both $\theta_{\mathrm{L}}$ and $\theta_{\mathrm{T}}$ were significantly depressed to $29 \pm 6.0$ and $11 \pm 4.4 \mathrm{~cm} / \mathrm{s}$ 


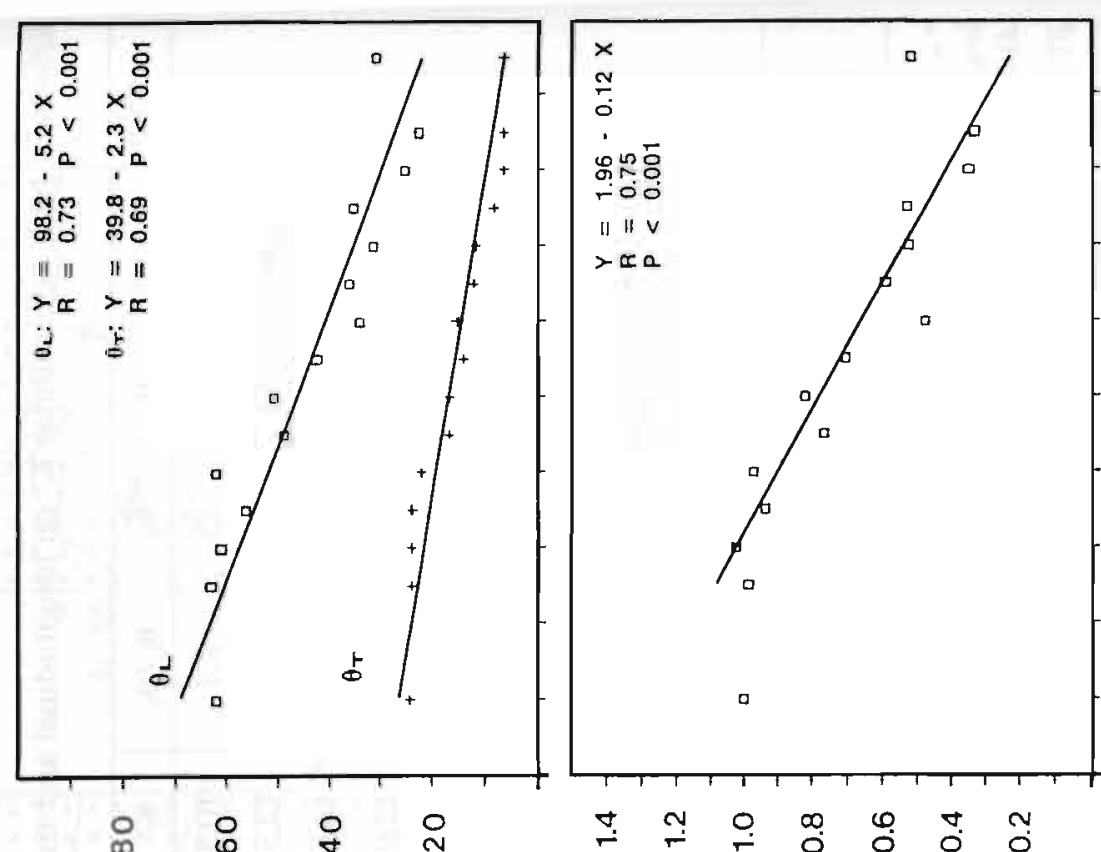

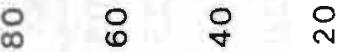

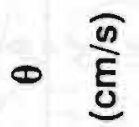

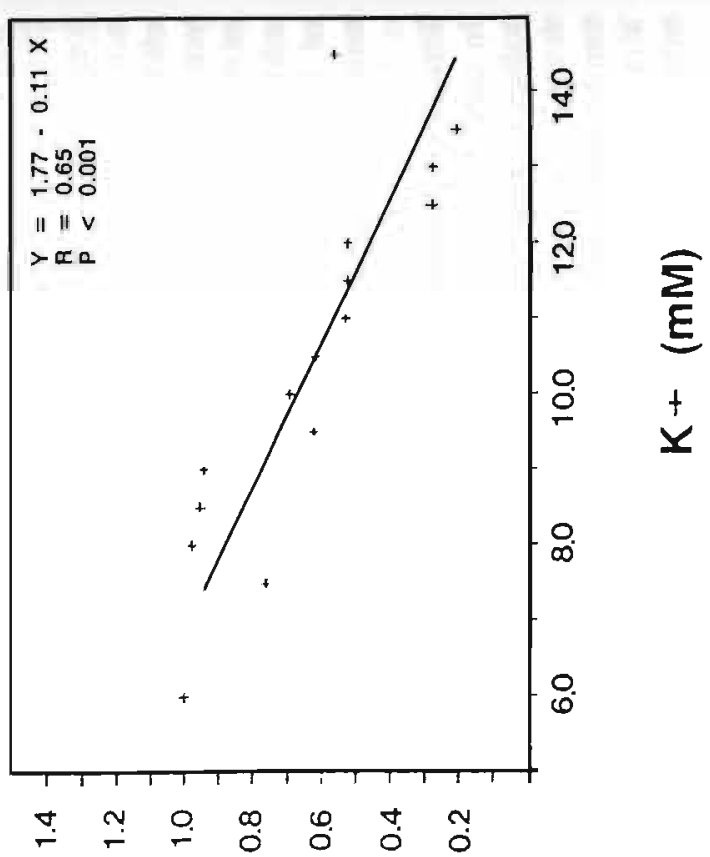

$\perp_{\theta} \quad$ a $3 Z I 7 \forall W Y O N$

Figure 5.4: Graphs representing absolute and normalized values of $\hat{\theta}_{L}$ and $\theta_{T}$ at increasing potassium concentration. In middle and lower panels the $\theta$ values 

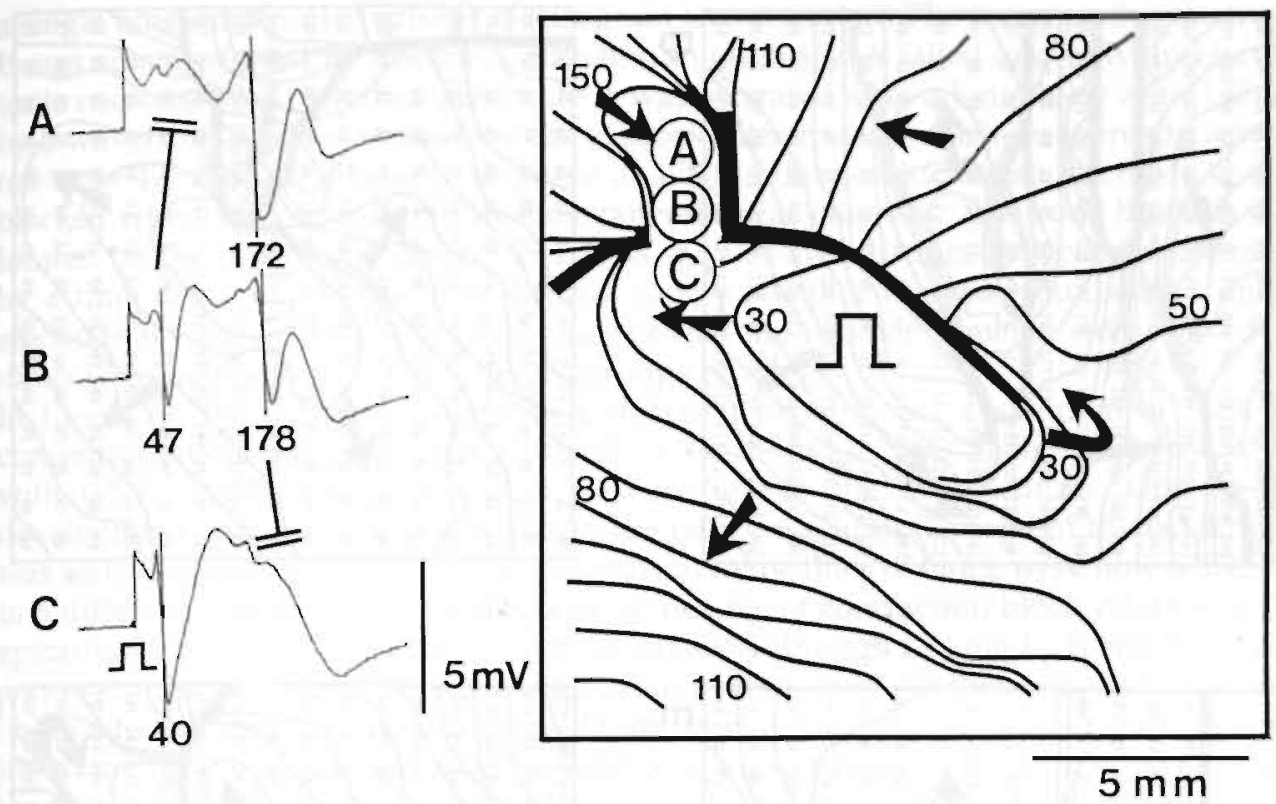

Figure 5.5: Activation map during slow pacing $(0.5 \mathrm{~Hz})$ at $11.0 \mathrm{mM}$ of $\mathrm{K}^{+}$showing a long arc of functional conduction block (thick black line). Electrograms $A, B$ and $C$ (interelectrode distance $1 \mathrm{~mm}$ ) were recorded across the line of block. The vertical lines superimposed on the electrograms indicate the moments of local activation. Numbers indicate the activation times relative to the stimulus. See text for description.

$(\mathrm{p}<0.001)$, with a ratio of $3.2 \pm 1.3(\mathrm{p}=0.26$, n.s.). These data show that despite the large degree of depression of conduction by $\mathrm{K}^{+}$no statistically significant difference could be detected between the effects of $\mathrm{K}^{+}$on longitudinal or transverse conduction. In figure 5.4 the average values of longitudinal and transverse conduction velocities are plotted for all potassium concentrations. In the upper panel the absolute values of $\theta_{\mathrm{L}}$ and $\theta_{\mathrm{T}}$ are given whereas in the middle and lower panel the values of $\theta_{\mathrm{L}}$ and $\theta_{\mathrm{T}}$ are normalized to their control value. Although the slope of the regression line of $\theta_{\mathrm{L}}$ is steeper than that of $\theta_{\mathrm{T}}$ (5.2 and 2.3 respectively, upper panel) the relative changes in conduction velocity were the same, the regression lines of the normalized values of $\theta_{\mathrm{L}}$ and $\theta_{\mathrm{I}}$ (middle and lower panel) having a slope of 0.12 and 0.11 respectively $(\mathrm{p}=0.02)$. Thus during slow pacing of a two dimensional sheet of perfused anisotropic ventricular epicardium, depression of conduction of more than $50 \%$ by increasing extracellular $\mathrm{K}^{\prime}$ did not result in a statistically significant change in the ratio of anisotropic conduction.

\section{Longitudinal and transverse conduction block}

Multiple local arcs of conduction block and areas of inexcitability were found at high $\mathrm{K}^{+}$concentrations before the ventricles became completely inexcitable. An example of the electrograms recorded across a line of block at $11.0 \mathrm{mM}$ of $\mathrm{K}^{+}$is given together with the activation map in figure 5.5. The distance between the electrodes labeled A, B and $C$ was $1 \mathrm{~mm}$. The longitudinal wavefront propagating to the left from the site of stimulation activated the area under electrode $C 40 \mathrm{~ms}$ after a stimulus was given and 

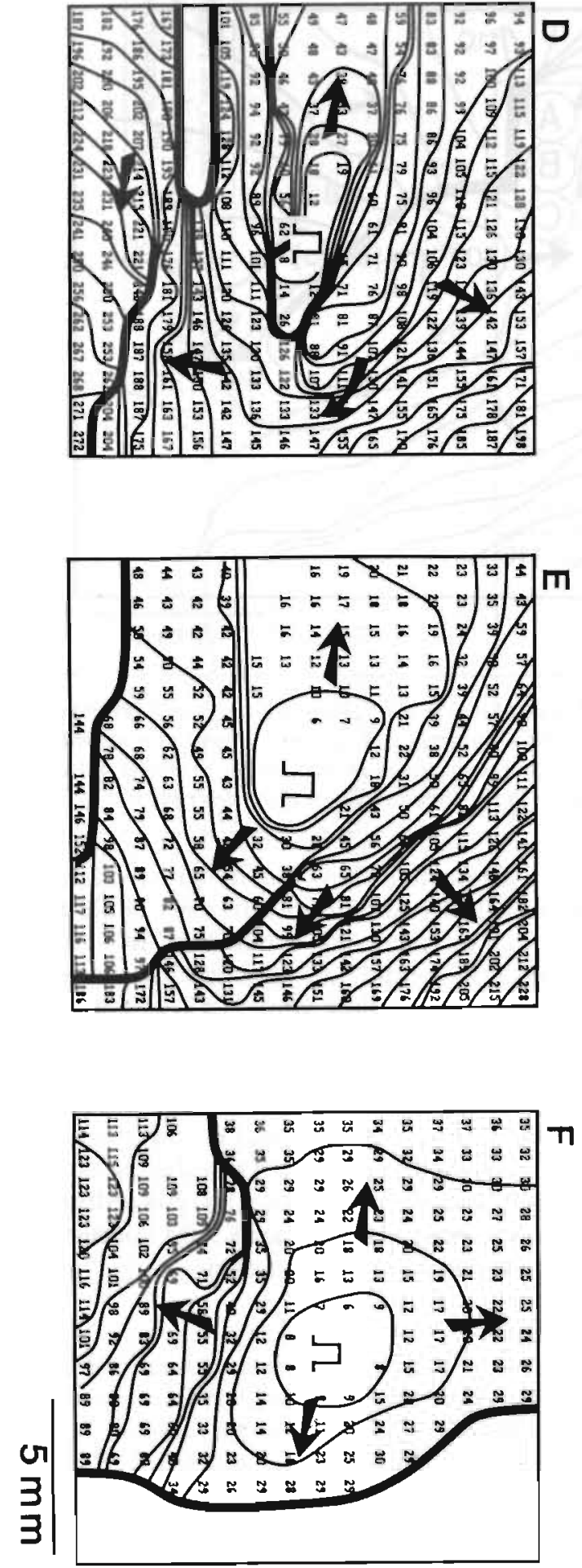

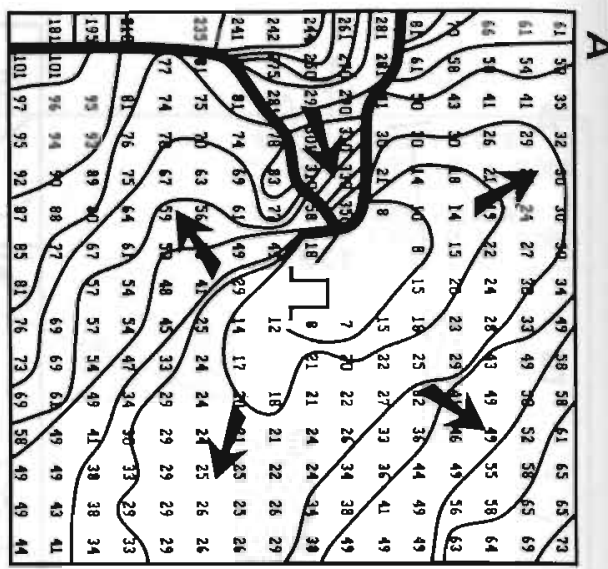

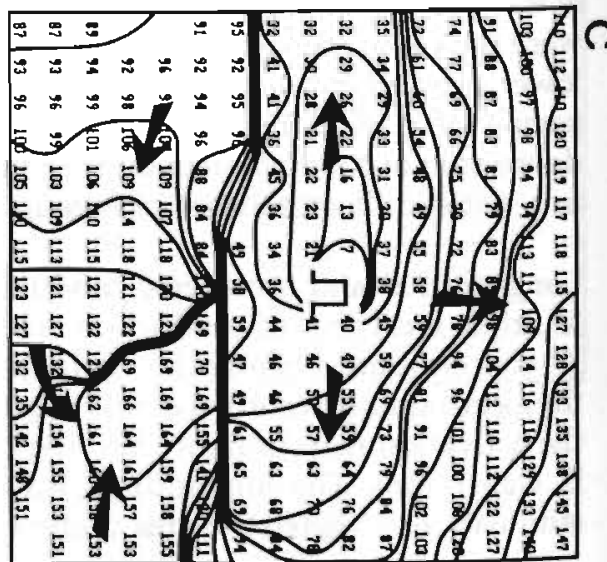


a single high amplitude biphasic electrogram was recorded. The tissue under electrode $B$ was activated after $47 \mathrm{~ms}$ but conduction to electrode A failed and only an electrotonic response was recorded. Electrode $A$ was activated after a long delay at $t=172 \mathrm{~ms}$ by a wavefront turning around the arc of block. Electrode B which was already activated at $t=47$, showed a second response at $178 \mathrm{~ms}$ but conduction to electrode $C$ was blocked where only a small electrotonic potential was recorded. Electrode B which was located on the line of functional block thus showed two distinct deflections separated by a time delay of $130 \mathrm{~ms}$. It is difficult to say whether the individual fibers under electrode B were actually activated twice or that the two components were caused by dissociated activation of two different groups of fibers.

In figure 5.6 the activation maps of 6 different experiments are shown at high $\mathrm{K}^{+}$ concentrations ranging between 11.0 and $14.5 \mathrm{mM}$. In all cases a slight increase of $\mathrm{K}^{+}$ with $0.5-1.0 \mathrm{mM}$ resulted in total inexcitability of the myocardium. Due to the development of local conduction blocks the uniform elliptical shape of the isochrones was seriously distorted because the areas distal to the lines of block were now activated in a different direction. The localization of the arcs of conduction block relative to the epicardial fiber orientation was erratic. In panels A through $\mathrm{C}$, long $\mathrm{L}, \mathrm{T}$, and $\mathrm{Y}$ shaped arcs of conduction block were present with limbs oriented parallel, perpendicular and oblique to the fiber direction. In panel $\mathrm{D}$ the narrow longitudinal wavefront propagating to the right was blocked while several long arcs of transverse block were found in the lower part of the mapping area. The example in panel $\mathrm{E}$ showed a marked increase in the ratio of anisotropy at high $\mathrm{K}^{+}$. Whereas a longitudinal wavefront still propagated at relatively high speed from the point of stimulation to the left $\left(\theta_{\mathrm{L}}=42 \mathrm{~cm} / \mathrm{s}\right)$ transverse propagation to the upper right corner was markedly depressed $\left(\theta_{\mathrm{T}}=4 \mathrm{~cm} / \mathrm{s}\right)$. In the lower right part of panel $\mathrm{E}$ a long line of block was present parallel to the fiber orientation dissociating two longitudinal wavefronts propagating in the same direction. In panel $\mathrm{F}$ a large area of inexcitability was present in the right part of the mapping area while other parts of the preparation were still conducting at relatively fast speed. In the lower left part of the map an S-shaped line of block was present with a short line of longitudinal block connecting two longer lines of transverse block.

In these experiments no consistent pattern in the localization of lines of functional conduction block relative to the fiber orientation could be found. This indicates that for the development of intramyocardial conduction block by increased extracellular potassium other spatial inhomogeneities in structural or electrophysiological properties are more important than uniform tissue anisotropy.

\section{DISCUSSION}

Several studies have been directed to elucidate the role of anisotropic tissue structure on intramyocardial conduction and conduction block. Spach et al. ${ }^{4}$ have shown that the

Figure 5.6 (left): Activation maps of 6 different experiments during slow regular pacing $(0.5 \mathrm{~Hz})$ at the center of the electrode array during high potassium. The concentrations of $K^{+}$in panels $A-F$ were $12.5,11.0$, $14.0,14.5,12.5$, and $13.5 \mathrm{mM}$ respectively. Activation times are given in milliseconds and isochrones are drawn at $10 \mathrm{~ms}$ intervals. Arrows indicate the direction of propagation and thick black lines represent functional conduction block with a local conduction delay of more than $40 \mathrm{~ms}$. See text for description. 
lower effective axial resistance parallel to the fiber orientation leads to a decrease of the upstroke velocity of the action potential because a larger amount of the generated current flows away through this low resistance "current sink" ahead of the propagating depolarization wave. Fast upstrokes were found during slow propagation transverse to the long axis of the cells because less of the current generating phase 0 of the action potential is used for electrotonic depolarization of the downstream membrane. Consequently the action potential during transverse propagation will be closer to the "genuine" membrane action potential and although proceeding at a lower speed might be more resistant to disturbances in the membrane ionic properties than fast longitudinal conduction. This would explain the observation that early premature wavefronts may block preferentially in longitudinal direction ${ }^{4}$.

In a later study ${ }^{5}$ comparing experimental studies with computer simulations, the direction-dependent differences in the shape of the action potential were attributed to hypothetical directional differences in the effective membrane capacitance. The combination of a higher effective membrane capacitance and a lower axial resistance parallel to the fiber orientation imposes a higher electrical load on the propagating wavefront and may result in a lower safety factor for longitudinal conduction. In a study performed in 1988, Spach et $a .^{6}{ }^{6}$ found that in uniform anisotropic canine ventricular preparations, premature beats decreased $\theta_{\mathrm{L}}$ more than $\theta_{\mathrm{T}}$ (by $46 \%$ and $36 \%$ respectively). However in contrast to their earlier studies ${ }^{4}$ conduction block occurred simultaneously both in longitudinal and transverse direction.

The directional effects of depressed active membrane properties by high extracellular $\mathrm{K}^{+}$on anisotropic conduction have been investigated in several studies. Tsuboi et al. ${ }^{7}$ found that increasing the extracellular $\mathrm{K}^{+}$from 4.0 to $11.0 \mathrm{mM}$ resulted in equal reduction of $\theta_{\mathrm{L}}$ and $\theta_{\mathrm{T}}$ (by $58 \%$ and $56 \%$ ). However conduction block occurred preferentially in a longitudinal direction. Raising the extracellular $\mathrm{K}^{+}$from 7.4 to 10.8 $\mathrm{mM}$, Kléber et al. ${ }^{15}$ found a greater depression of $\theta_{\mathrm{L}}$ than $\theta_{\mathrm{T}}$ (by $35 \%$ and $22 \%$, n.s.). In contrast in one-dimensional L-shaped sheep epicardial preparations Delgado et al. ${ }^{12}$ reported a greater depression of $\theta_{\mathrm{T}}$ than $\theta_{\mathrm{L}}$ (by $47 \%$ and $20 \%$ ) when extracellular $\mathrm{K}^{+}$was raised from 4.0 to $20.0 \mathrm{mM}$. Conduction block occurred first in a transverse direction in 8 out of 9 preparations. Since other studies ${ }^{16,17}$ had demonstrated that transverse conduction was preferentially affected when the intercellular coupling resistance was increased by Heptanol they concluded that regardless of whether intercellular coupling or active generator properties are impaired, conduction in transverse direction is more vulnerable to conduction disturbances. ${ }^{12}$

In our experiments on two-dimensional anisotropic conduction in sheets of perfused rabbit epicardium, we failed to establish a consistent differential effect of high $\mathrm{K}^{+}$on transverse or longitudinal conduction. To avoid the possible occurrence of supernormal conduction at low $\mathrm{K}^{+18}$, potassium concentrations higher than $6.0 \mathrm{mM}$ were used. The stimulation frequency was chosen as low as $0.5 \mathrm{~Hz}$ to exclude rate dependent effects on anisotropic conduction. ${ }^{7}$ Under these conditions, $\theta_{\mathrm{L}}$ and $\theta_{\mathrm{T}}$ were about equally depressed by $52 \%$ and $56 \%$. The concommittent slight increase in the ratio of anisotropy was not statistically significant Arcs of functional conduction block were found both in longitudinal and transverse direction. Although $\mathrm{K}^{+}$was gradually raised in small steps of $1.0 \mathrm{mM}$ we could not find a certain concentration of $\mathrm{K}^{+}$where block systematically occurred either in one or the other direction. These results are in agreement with the studies of Tsuboi et al. ${ }^{7}$ and Kléber et al. $^{15}$, although they do not explain why preferential longitudinal conduction block occurred in the experiments of Tsuboi et al. ${ }^{7}$ 
Our results differ from the recent findings of Delgado et al. ${ }^{12}$ In their study thin isolated L-shaped strips of sheep epicardium were studied during superfusion in a tissue bath. At a temperature of $37^{\circ} \mathrm{C}$ and a $\mathrm{K}^{+}$concentration of $4.0 \mathrm{mM}$, these preparations exhibited a large variation both in longitudinal and transverse conduction velocity $\left(\theta_{\mathrm{L}}\right.$ : range $10-92 \mathrm{~cm} / \mathrm{s}$, mean $50 \pm 23 \mathrm{~cm} / \mathrm{s} ; \theta_{\mathrm{T}}$ : range $6-14 \mathrm{~cm} / \mathrm{s}$, mean $9 \pm 3 \mathrm{~cm} / \mathrm{s} ; \mathbf{n}=9$ ). The conduction velocity in the transverse limb of the L-shaped preparation of $9 \mathrm{~cm} / \mathrm{s}$ was considerably less than the normal $\theta_{\mathrm{T}}$ of $25 \mathrm{~cm} / \mathrm{s}$ in perfused ventricular myocardium. As a result, the ratio of anisotropy in these preparations was more than twice the value under normal physiological conditions. This raises the question whether in these narrow pathways, transverse conduction was slowed down by a reduction of the available gap junctions, or whether depressed transverse conduction was due to a varying degree of tissue damage by the procedure to create the preparations. If for any of these reasons the safety factor for transverse conduction was already decreased during control, this might explain that elevation of extracellular $\mathrm{K}^{+}$produced preferential transverse conduction block in 8 of 9 preparations.

The isolated perfused rabbit epicardium used in our study showed normal values of $\theta_{\mathrm{L}}$ and $\theta_{\mathrm{T}}$ even at very high heart rates. ${ }^{19}$ However, in a bidimensional sheet of myocardium, effective transverse propagation will occur through the fastest possible pathway available, either in a zig-zag diagonal way or by true side-to-side transverse conduction. Transverse conduction is therefore less vulnerable to small conduction disturbances that can have a large effect in one-dimensional preparations. These differences between our model and the model used by Delgado et al. ${ }^{12}$ emphasize the importance of using one-, two-, or three-dimensional preparations to elucidate the role of anisotropic tissue structure on impulse conduction under various circumstances.

We conclude that in a perfused 2-D sheet of ventricular epicardium a decrease in generated membrane current by raising extracellular $\mathrm{K}^{+}$does not result in preferential conduction block related to the epicardial fiber orientation. Even if differences in safety factor exist between longitudinal and transverse conduction, these differences are so small that they are completely overruled by other more important spatial inhomogeneities in structural or electrophysiological conduction properties.

\section{CONDENSED ABSTRACT}

In a thin layer of Langendorff perfused epicardium, the effects of depressed active membrane properties on anisotropic impulse propagation were studied by raising the extracellular $\mathrm{K}^{+}$concentration during central stimulation at low heart rates. Elevation of the extracellular $\mathrm{K}^{+}$concentration decreased conduction velocity both in a longitudinal and in a transverse direction but the ratio of anisotropy was not changed. At very high concentrations of $\mathrm{K}^{+}$arcs of local conduction block appeared both in a longitudinal and in a transverse direction. From these results we conclude that during slow heart rates, depression of the fast sodium channels by high extra-cellular $\mathrm{K}^{+}$causes no detectable differential effects on conduction velocity or on the occurrence of conduction block longitudinal or transverse to the general fiber orientation. 


\section{REFERENCES}

1. Sano T, Takayama N, Shimamoto T: Directional difference of conduction velocity in the cardiac ventricular syncytium studied by microelectrodes. Circ Res 1959;7:262-268

2. Clerc L: Directional differences of impulse spread in trabecular muscle from mammalian heart. J Physiol 1976;255:335-346

3. Hodgkin AL: A note on conduction velocity. J Physiol 1954;125:221-224

4. Spach MS, Miller III WT, Geselowitz DB, Barr RC, Kootsey JM, Johnson EA: The discontinuous nature of propagation in normal canine cardiac muscle. Evidence for recurrent discontinuities of intracellular resistance that effect the membrane currents. Circ Res 1981;48:39-54

5. Spach MS, Dolber PC, Heidlage JF, Kootsey JM, Johnson EA: Propagating depolarization in anisotropic human and canine cardiac muscle: Apparent directional differences in membrane capacitance. A simplified model for selective directional effects of modifying the sodium conductance on $V_{\max }, T_{\text {foot }}$ and the propagation safety factor. Circ Res 1987;60:206-219

6. Spach MS, Dolber PC, Heidlage JF: Influence of the passive anisotropic properties on directional differences in propagation following modification of the sodium conductance in human atrial muscle. A model of reentry based on anisotropic discontinuous propagation. Circ Res $1988 ; 62: 811-832$

7. Tsuboi N, Kodama I, Toyama J, Yamada K: Anisotropic conduction properties of canine ventricular muscles-influence of high extracellular $\mathrm{K}^{+}$concentration and stimulation frequency. Jpn Circ J 1985;49:487-498

8. Kadish AH, Spear JF, Levine JH, Moore EN: The effects of procainamide on conduction in anisotropic canine ventricular myocardium. Circulation 1986;74:616-625

9. Osaka T, Kodama I, Tsuboi N, Toyama J, Yamada K: Effects of activation sequence and anisotropic cellular geometry on the repolarization phase of action potential of dog ventricular muscles. Circulation 1987;76:226-236

10. Van Capelle FJM: Slow conduction and cardiac arrhythmias (thesis). Amsterdam, The Netherlands, University of Amsterdam 1983, pp 127-166

11. Delmar M, Delgado C, Chialvo D, Michaels DC, Jalife J: On the problem of anisotropic propagation in ventricular muscle. In: Rosen MR, Palti $Y$, ed. Lethal Arrhythmias Resulting from Myocardial Ischaemia and Infarction. Kluwer Academic Publishers, Boston/Dordrecht/London, 1989:p.181

12. Delgado C, Steinhaus B, Delmar M, Chialvo DR, Jalife J: Directional differences in excitability and margin of safety for propagation in sheep ventricular epicardial muscle. Circ Res 1990;67:97-110

13. Gordon $\mathrm{H}$ and Sweets HH: Simple method for the silver impregnation of reticulum. Am J Pathol $1936 ; 12: 545-552$

14. Dillon SM, Allessie MA, Ursell PC, Wit AL: Influences of anisotropic tissue structure on reentrant circuits in the epicardial border zone of subacute canine infarcts. Circ Res 1988:63:182-206

15. Kléber AG, Janse MJ, Wilms-Schopmann FJG, Wilde AM, Coronel R: Changes in conduction velocity during acute ischemia in ventricular myocardium of the isolated porcine heart. Circulation 1986;73:189-198.

16. Delmar M, Michaels DC, Johnson T, Jalife J: Effects of increasing intercellular resistance on transverse and longitudinal propagation in sheep epicardial muscle. Circ Res 1987;60:780-785

17. Balke CW, Lesh MD, Spear JF, Kadish A, Levine JH, Moore EN: Effects of cellular uncoupling on conduction in anisotropic canine ventricular myocardium. Circ Res 1988;63:879-892

18. Dominguez $\mathrm{G}$ and Fozzard $\mathrm{H}$ : Influence of extracellular $\mathrm{K}^{+}$concentration on cable properties and excitability of sheep cardiac Purkinje fibers. Circ Res 1970;26:565-574

19. Brugada J, Boersma L, Kirchhof C, Brugada P, Havenith M, Wellens HJ, Allessie MA: Doublewave reentry as a mechanism of acceleration of ventricular tachycardia. Circulation 1990;81:16331643 


\section{CHAPTER 6}

THE EFFECTS OF $\mathrm{K}^{+}$ON VENTRICULAR

TACHYCARDIA AROUND A FIXED ANATOMICAL OBSTACLE.

In part: Circulation 1991;84:1307-1318 


\section{INTRODUCTION}

Due to the ultrastructure of cardiac myocytes, ${ }^{1,2}$ conduction parallel to the orientation of the fibers is fast while transverse to the fiber axis slow conduction occurs. ${ }^{3.4}$ In the intact left ventricle conduction is fast in every direction, due to the three-dimensional structure of ventricular myocardium and the 120 degree change in fiber orientation from endocardium to epicardium. ${ }^{5}$ However, after myocardial infarction, a thin 2-D border zone of the infarct may survive in which the anisotropic conduction properties of ventricular myocardium become apparent and are even enhanced due to uncoupling of adjacent muscle bundles by fibrosis. ${ }^{6-8}$ This abnormal border zone has been found to be part of the substrate for reentrant arrhythmias after myocardial infarction. ${ }^{9-11}$ It can be expected that during reentrant VT the circulating impulse will propagate parallel to the fiber orientation in some segments of the circuit and perpendicular in others. In this study, we evaluated whether a depression of the upstroke of the action potential by high concentrations of extracellular $\mathrm{K}^{+}$would slow and terminate reentrant VT by a preferential effect on either longitudinal or transverse conduction.

\section{METHODS}

\section{Preparation}

In 18 Langendorff-perfused rabbit hearts a thin layer of left ventricular subepicardium was created by cryoprocedure. The perfusion system and the cryoprocedure have been described in detail in chapter 2 . In the thin epicardial layer, a fixed anatomical obstacle $(25 \times 10 \mathrm{~mm})$ was created, as described in chapter 4 .

\section{Recording and Stimulation}

A spoon-shaped mapping electrode, molded to the epicardial surface of the left ventricle, containing 248 individual silver electrodes (diameter $0.3 \mathrm{~mm}$, resolution 2.25 $\mathrm{mm}$ ), was used to map the ventricular activation (chapter 3 ). Bipolar stimulation could be performed through any pair of electrodes in the electrode spoon. Ventricular tachycardia around the central obstacle was induced by applying up to three extrastimuli (see chapter 4 ). Longitudinal conduction velocity $\left(\theta_{L}\right)$ was determined at the base and free wall of the left ventricle while transverse conduction velocity $(\theta \mathrm{T})$ was measured at the corridor between the LAD and central obstacle (chapter 4). Conduction velucity was calculated as the distance travelled normal to the isochrones per unit time.

\section{Infusion of $\mathrm{K}^{+}$during VT}

The concentration of $\mathrm{K}^{+}$in the Tyrode's solution was raised with steps of $1.0 \mathrm{mM}$ by infusion of an iso-osmotic solution with a high concentration of $\mathrm{K}^{+}$. An equilibration time of 10 minutes was allowed before a higher concentration of $\mathrm{K}^{+}$was tested. At each concentration a sample of the perfusate was taken from the outflow of the heart. After the experiment the exact concentration of $\mathrm{K}^{+}$was determined by a flame-photometer (model 243, Fisher Medical Division Instrumentation Laboratory, Lexington, MA, USA).

\section{Statistical Analysis}

Results were compared using paired Students' t-test. P-values below 0.05 were taken as statistically significant. 


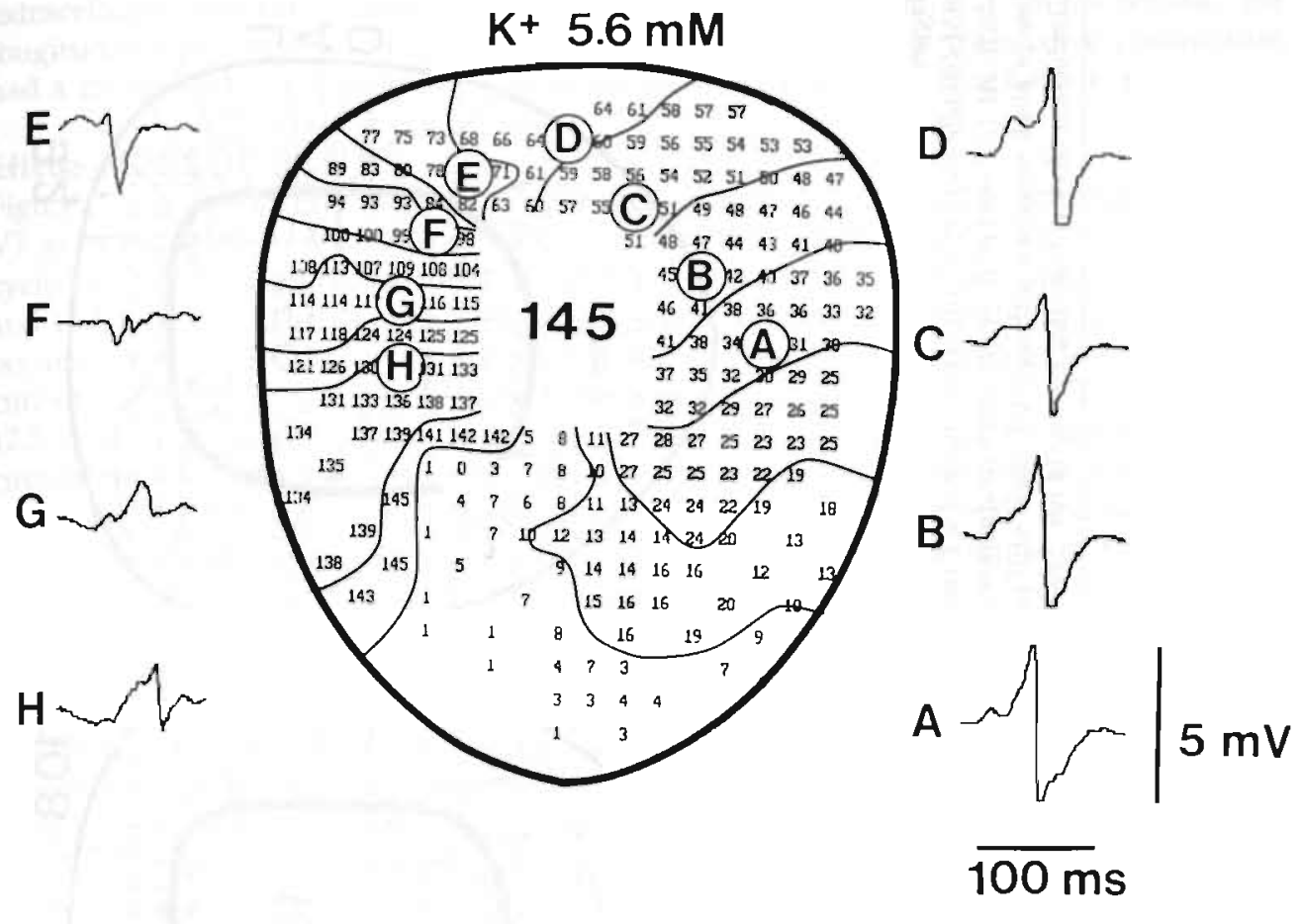

Figure 6.1: Activation map of reentrant ventricular tachycardia around a fixed anatomical obstacle. At 5.6 $m M$ of $\mathrm{K}^{+}$the counterclockwise circulating wave had a revolution time of $145 \mathrm{~ms}$. In the corridor between the LAD and the central obstacle conduction tranverse to the epicardial fiber orientation was slow $(25 \mathrm{~cm} / \mathrm{s}$ ) while longitudinal conduction at the base and the free wall was fast $(64 \mathrm{~cm} / \mathrm{s})$. The electrograms left and right of the activation map were recorded at sites $A-H$ indicated in the map. Electrograms $A-D$ (right) were characteristic for longitudinal impulse conduction while electrograms $E-H$ (left) were typical for transverse conduction. Activation times are given in milliseconds and isochrones are drawn at $10 \mathrm{~ms}$ intervals.

\section{RESULTS}

\section{Characteristics of Ventricular Tachycardia}

In 18 experiments programmed electrical stimulation induced reentrant ventricular tachycardia (VT) around the central obstacle. VT was based either on a clockwise $(\mathrm{N}=12)$ or a counterclockwise $(\mathrm{N}=6)$ circulating wave. During control at an extracellular $\mathrm{K}^{+}$of $5.6 \mathrm{mM}$ the average cycle length of the $18 \mathrm{VTs}$ was $148 \pm 19 \mathrm{~ms}$ (range 118 to 198 $\mathrm{ms})$. An example of VT during control is shown in figure 6.1. In the activation map the reentrant impulse circulated around the obstacle in a counterclockwise direction with a revolution time of $145 \mathrm{~ms}$. In the corridor between the LAD and the central obstacle the impulse propagated slowly $\left(\theta_{\mathrm{T}}=25 \mathrm{~cm} / \mathrm{s}\right)$ transverse to the epicardial fiber orientation. At the base and free wall of the left ventricle propagation parallel to the fiber orientation $\left(\theta_{\mathrm{L}}\right)$ was much faster $(68 \mathrm{~cm} / \mathrm{s})$. The electrograms A-D and $\mathrm{E}-\mathrm{H}$ given at the right and left side of the activation map show the local extracellular wave forms in different segments of the ring during VT. At the base and free wall (A-D) the 
extracellular potentials were biphasic and of high amplitude, characteristic for longitudinal propagation. Electrograms $\mathrm{E}-\mathrm{H}$ in the segment of transverse conduction had a much lower amplitude and were bi- or multiphasic (electrograms F and G).

\section{Effects of high $\mathrm{K}^{+}$on VT}

Figure 6.2 shows the effects of increasing the extracellular $\mathrm{K}^{+}$concentration on the same VT as in figure 6.1. As $\mathrm{K}^{+}$was gradually raised from 5.6 to $9.2,10.8$, and $12.2 \mathrm{mM}$, the cycle length of VT increased from $145 \mathrm{~ms}$ to 160,187 , and $278 \mathrm{~ms}$ respectively. At 9.2 and $10.8 \mathrm{mM}$ of $\mathrm{K}^{+}$the impulse still propagated uniformly around the ring without the occurrence of local conduction block or areas of inexcitability. $\theta_{\mathrm{L}}$ had slowed from 68 $\mathrm{cm} / \mathrm{s}$ to 59 and $53 \mathrm{~cm} / \mathrm{s}$ while $\theta_{\mathrm{T}}$ was decreased from $25 \mathrm{~cm} / \mathrm{s}$ to 24 and $23 \mathrm{~cm} / \mathrm{s}$. At $12.2 \mathrm{mM}$ of $\mathrm{K}^{+}$the VT had significantly slowed to $278 \mathrm{~ms}$ and conduction of the circulating wave was seriously impaired at some sites in the ventricle, indicated by crowding of more than 2 isochrones between adjacent electrodes. $\theta_{\mathrm{L}}$ and $\theta_{\mathrm{T}}$ had both markedly decreased to 23 and $14 \mathrm{~cm} / \mathrm{s}$ respectively. At the entrance of the corridor between the LAD and the obstacle a small line of partial conduction block had developed without terminating the circulating wave.

Table 6.1 shows the average effects of high extracellular $\mathrm{K}^{+}$on the longitudinal and transverse conduction velocities during 18 VTs. The control values for $\theta_{\mathrm{l}}$ and $\theta_{\mathrm{T}}$ were determined at $5.6 \mathrm{mM}$ of $\mathrm{K}^{+}$while the values during high extracellular $\mathrm{K}^{+}$were determined the last beat before termination of VT. In 18 experiments during control the cycle length of VT was $148 \pm 19 \mathrm{~ms}$. The $\theta_{\mathrm{L}}$ of $67 \pm 11 \mathrm{~cm} / \mathrm{s}$ clearly exceeded the $\theta_{\mathrm{T}}$ of $26 \pm 13 \mathrm{~cm} / \mathrm{s}(\mathrm{p}<0.001)$, resulting in an anisotropy ratio of $2.9 \pm 0.7$. At an average extracellular $\mathrm{K}^{+}$of $11.9 \pm 1.3 \mathrm{mM}$ the cycle length of VT had markedly increased to 348 $\pm 156 \mathrm{~ms}$. Both $\theta_{\mathrm{L}}$ and $\theta_{\mathrm{T}}$ had been decreased significantly ( $\mathrm{p}<0.001$ ) to $25 \pm 7$ and $16 \pm 7$ $\mathrm{cm} / \mathrm{s}$ respectively. High extracellular $\mathrm{K}^{+}$preferentially depressed longitudinal impulse propagation, decreasing the anisotropy ratio $\left(\theta_{\mathrm{L}} / \theta_{\mathrm{T}}\right)$ to $1.9 \pm 0.9(\mathrm{p}<0.001)$.

\section{Termination of VT by high $\mathrm{K}^{+}$}

In all $18 \mathrm{VTs}$ at high concentrations of $\mathrm{K}^{+}$conduction block of the circulating wave

\section{Table 6.1:}

Effects of high $\mathrm{K}^{+}$on Reentrant Ventricular Tachycardia ( $\left.\mathrm{n}=18\right)$.

\begin{tabular}{ccc}
\hline & Control & Termination of VT \\
\hline $\mathrm{K}^{+}$ & 5.6 & $11.9 \pm 1.3^{*}$ \\
Cycle Length & $148 \pm 19$ & $348 \pm 156^{*}$ \\
$\theta_{\mathrm{L}}$ & $67 \pm 11$ & $25 \pm 7^{*}$ \\
$\theta_{\mathrm{T}}$ & $26 \pm 13$ & $16 \pm 7^{*}$ \\
$\theta_{\mathrm{L}} / \theta_{\mathrm{T}}$ & $2.9 \pm 0.7$ & $1.9 \pm 0.9^{*}$ \\
\hline
\end{tabular}

${ }^{*}=p<0.001 ;\left[\mathrm{K}^{+}\right]=$extracellular potassium concentration $(\mathrm{mM}) ; \theta_{L}=$ longitudinal conduction velocity (cm/s); $\theta_{T}=$ transverse conduction velocity $(\mathrm{cm} / \mathrm{s}) ; \theta_{V} / \theta_{T}=$ ratio of anisotropy. 

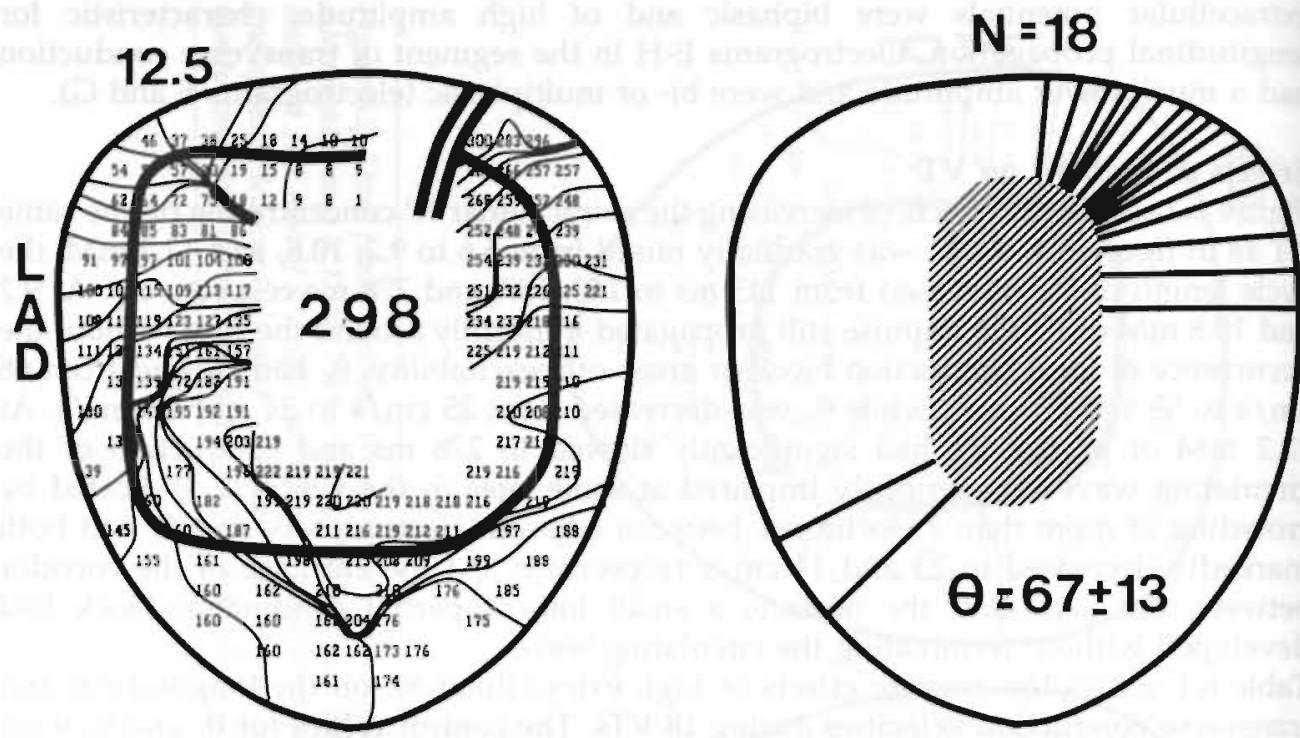

Figure 6.3: Left: Termination of (same as in figures 5.1 and 5.2) at $12.5 \mathrm{mM}$ of $\mathrm{K}^{*}$. Prior to termination the cycle length of VT had increased to $298 \mathrm{~ms}$. The circulating wave was completely blocked at the base of the left ventricle and VT was terminated. Activation times are given in milliseconds and isochrones are drawn at $10 \mathrm{~ms}$ intervals. Bars indicate conduction block. Right: Sites of conduction block during termination of $V T(n=18)$. In 16 of 18 hearts the area of block was located at the base. During control, the average conduction velocity at the segment of termination of VT was $67 \pm 13 \mathrm{~cm} / \mathrm{s}$.

resulted in termination of VT. The left panel of figure 6.3 gives an example of termination of the same VT as in figures 6.1 and 6.2. During the last beat of VT at $12.5 \mathrm{mM}$ of $\mathrm{K}^{+}$, the counterclockwise circulating wave propagated around the central obstacle with a revolution time of $298 \mathrm{~ms}$. However, as the circulating wave propagated from the free wall to the base of the left ventricle conduction was blocked across the entire width of the ring and VT was terminated. During control (figure 6.1), in the segment of the ring where VT was terminated, the conduction velocity was fast (68 $\mathrm{cm} / \mathrm{s}$ ) and the local electrograms were characteristic for longitudinal conduction. The right panel of figure 6.3 shows that in 16 of 18 terminations the line of conduction block was located at the base or free wall of the left ventricle, while in two cases conduction block occurred at the apex. At the site of termination of VT, during control the conduction velocity was $67 \pm 13 \mathrm{~cm} / \mathrm{s}(\mathrm{n}=18)$ ranging from 42 to $87 \mathrm{~cm} / \mathrm{s}$. In 10 hearts the site of conduction block during termination of VT by high $\mathrm{K}^{+}$was compared to the site of unidirectional block during initiation of VT by extrastimuli as described in chapter 4 . In 9 of 10 cases the site of conduction block during initiation or termination of VT was exactly the same. Comparison of histologic data with the activation maps revealed that in this segment of the ring the impulse propagated parallel to the fiber orientation. Thus, it seems that conduction block during VT by high extracellular $\mathrm{K}^{+}$ occurs preferentially when the impulse propagates longitudinal to the fiber orientation. Spatial dispersion in refractoriness did not seem to be involved in conduction block. 
During regular pacing $(\mathrm{S} 1-\mathrm{S} 1=250 \mathrm{~ms}$ ) in 6 of 10 hearts the refractory period distal to the area of block was equal to or shorter than $(-9 \pm 6 \mathrm{~ms})$ the refractory period proximal to this area. Only in 4 VTs the refractory period distal to the line of block exceeded the refractory period proximal to the site of block $(+10 \pm 7 \mathrm{~ms})$. Thus, in at least 6 of 10 hearts spatial dispersion in refractoriness was not involved in the development of conduction block. The fact that both premature stimuli and high extracellular $\mathrm{K}^{+}$ preferentially depressed conduction parallel to the fiber orientation strongly suggests that anisotropy was responsible for the occurence of conduction block.

\section{DISCUSSION}

In this study, the extracellular concentration of $\mathrm{K}^{+}$was raised to evaluate the effects of a depression of the active membrane properties on anisotropic impulse propagation during VT. It was assumed that the depression of conduction by elevation of $\mathrm{K}^{+}$was primarily due to a decrease in membrane resting potential, resulting in reduction of the $\mathrm{g}_{\mathrm{Na}}$ and of the reactivation kinetics of the sodium channel system. Gettes ${ }^{12}$ previously reported that at extracellular $\mathrm{K}^{+}$concentrations above $9 \mathrm{mM}$, slowing of conduction is mainly due to inactivation of the fast sodium channels. To our knowledge there is no evidence that high $\mathrm{K}^{+}$changes the axial resistance. ${ }^{13}$

Spach et al. ${ }^{14,15}$ have postulated that a decrease in active membrane properties would preferentially affect impulse propagation parallel to the fiber orientation, due to a lower safety factor for conduction compared to transverse propagation. Several studies have provided evidence favouring a preferential depression of ongitudinal conduction by high $\mathrm{K}^{+}{ }^{16,17}$ In this study we show that high concentrations of extracellular $\mathrm{K}^{+}$decrease the ratio for anisotropy and terminate reentrant VT by preferential longitudinal conduction block, which is in agreement with the hypothesis of Spach et al. ${ }^{14,15}$

\section{Different effects of high $\mathrm{K}^{+}$during slow pacing and VT}

The preferential effect of high $\mathrm{K}^{+}$on longitudinal conduction during VT is at odds with the results we have obtained during regular pacing at low frequencies in the intact 2-D layer (chapter 5). During control, at an extracellular concentration of $\mathrm{K}^{+}$of $5.6 \mathrm{mM}$, the conduction velocities during slow pacing in the sheet and during VT in the ring were comparable. The concentration of $\mathrm{K}^{+}$at which conduction block occurred during slow pacing was also similar to that during termination of VT. Nevertheless, during high extracellular $\mathrm{K}^{+}$in the intact epicardial layer we did not find a preferential effect of high $\mathrm{K}^{+}$on anisotropic conduction.

Rapid pacing has been found to preferentially depress transverse conduction, ${ }^{16,17}$ attributed to intracellular $\mathrm{Ca}^{++}$accumulation ${ }^{21}$ associated with an increase in intercellular resistance. Several investigators have shown that the refractory period during transverse conduction is longer than during longitudinal impulse propagation. ${ }^{1920} \mathrm{On}$ the basis of these observations one would expect that during VT transverse conduction would be preferentially affected by high $\mathrm{K}^{+}$, contrary to the preferential depression of longitudinal propagation which we observed.

During VT, conduction block was defined as block of the circulating wave leading to termination of VT. In the experiments in the intact epicardial sheet during slow pacing at high $\mathrm{K}^{+}$conduction block was defined as a time delay of more than $40 \mathrm{~ms} / \mathrm{mm}$ (apparent conduction velocity $<2.5 \mathrm{~cm} / \mathrm{s}$ ). It can be argued that the critical conduction 
velocity at which longitudinal block occurs is higher than $2.5 \mathrm{~cm} / \mathrm{s}$. If so, the incidence of longitudinal block during slow pacing may have been underestimated. However, this can still not explain the different effects on the ratio of anisotropy during both slow pacing and during ventricular tachycardia.

Taking everything together we cannot provide a satisfactory explanation for the different effects of high $\mathrm{K}^{+}$on anisotropic conduction during slow pacing and during $\mathrm{VT}$ in the ring.

\section{Clinical Implications}

The reentrant circuit in clinical VTs comprises an area of abnormal slow anisotropic conduction, ${ }^{6,710}$ which is considered to be a weak link of the reentrant pathway and the target for ablative therapy of VT. The ideal anti-arrhythmic drug should preferentially affect this area of slow conduction without depressing conduction in normal myocardium and should terminate VT at a low dose. In our experiments, sodiumchannel blocking by elevating the extracellular $\mathrm{K}^{+}$concentration during VT did not exert this effect. High extracellular $\mathrm{K}^{+}$preferentially slowed fast longitudinal conduction and only terminated VT after normal anisotropic conduction was slowed to less than $40 \%$ of its control value. This may explain why in some clinical VTs therapeutic dosages of sodium-channel blocking agents do not provide an adequate treatment for VT.

\section{CONDENSED ABSTRACT}

The effects of depression of the fast sodium channels on anisotropic conduction were tested by raising the extracellular $\mathrm{K}^{+}$concentration during reentrant ventricular tachycardia in a thin ring of perfused epicardium. During elevation of extracelllar $\mathrm{K}^{+}$ the cycle length of VT increased, due to a general slowing of conduction velocity. However, longitudinal conduction velocity was depressed more than conduction transverse to the epicardial fiber orientation, resulting in a decrease in the ratio of anisotropy. At very high $\mathrm{K}^{+}$concentrations VT was terminated by conduction block of the circulating impulse. Conduction block predominantly occurred when the impulse propagated longitudinal to the epicardial fiber orientation. From these results we conclude that during reentrant ventricular tachycardia in a thin epicardial ring, a depression of the fast sodium channels preferentially affects longitudinal impulse propagation due to a lower safety factor for conduction compared to transverse impulse propagation. 


\section{REFERENCES}

1. Clerc L: Directional differences of impulse spread in trabecular muscle from mammalian heart. J Physiol 1976;255:335-346

2. Sommer J, Johnson E. Ultrastructure of cardiac muscle. In Berne R, Sperelakis N, Geiger S (Eds): Handbook of physiology. Bethesda, American Physiological Society, 1979, pp 113-186

3. Sano T, Takayama N, Shimamoto T: Directional difference of conduction velocity in the cardiac ventricular syncytium studied by microelectrodes. Circ Res 1959;7:262-268

4. Schalij M, Lammers W, Rensma P, Allessie M: Anisotropic conduction and reentry in perfused epicardium of rabbit left ventricle. Am J Physiol 1992;263:H1466-1478

5. Streeter D. Gross morphology and fiber geometry of the heart. In: Handbook of physiology. Eds. Berne R, Sperilakis N, Geiger S, Bethesda, American Physiological Society, 1979, pp 61-112

6. Karagueuzian K, Fenoglio J, Weiss M, Wit A. Protracted ventricular tachycardia induced by premature stimulation of the canine heart after coronary artery occlusion and reperfusion. Circulation 1979;44:833-846

7. Michelson E, Spear J, Moore E. Electrophysiologic and anatomic correlates of sustained ventricular tachyarrhythmias in a model of chronic myocardial infarction. Am J Cardiol 1980;45:583-590

8. Spach M, Dolber P. Relating extracellular potentials and their derivatives to anisotropic propagation at a microscopic level in human cardiac muscle. Evidence for uncoupling of side-toside fiber connections with age. Circ Res 1986;58:356-371

9. Wit A, Allessie M, Bonke F, Lammers W, Smeets J, Fenoglio J. Electrophysiologic mapping to determine the mechanism of experimental ventricular tachycardia initiated by premature impulses. Experimental approach and initial results demonstrating reentrant excitation. Am J Cardiol 1982;49:166-185

10. Mehra R, Zeiler R, Gough W, El-Sherif N. Reentrant ventricular arrhythmias in the late myocardial infarction period. 9. Electrophysiologic-anatomic correlation of reentrant circuits. Circulation 1983;67:11-23

11. Dillon S, Allessie M, Ursell P, Wit A. Influences of aniostropic tissue structure on reentrant circuits in the epicardial border zone of subacute canine infarcts. Circ Res 1988;63:182-206

12. Gettes L. Effects of ionic changes in impulse propagation. In: Cardiac Electrophysiology. Eds Jalife J, Zipes D, Mount Kisco, NY, Futura Publishing Co, 1990, pp 459-479

13. Dominguez $\mathrm{G}$, Fozzard $\mathrm{H}$. Influence of extracellular $\mathrm{K}^{+}$concentration on cable properties and excitability of sheep cardiac Purkinje fibers. Circ Res 1970;26:565-574

14. Spach MS, Miller III WT, Geselowitz DB, Barr RC, Kootsey JM, Johnson EA: The discontinuous nature of propagation in normal canine cardiac muscle. Evidence for recurrent discontinuities of intracellular resistance that effect the membrane currents. Circ Res 1981;48:39-54

15. Spach M, Kootsey J, Sloan J. Active modulation of electrical coupling between cardiac cells of the dog. A mechanism for transient and steady-state variations in conduction velocity. Circ Res 1982:51:347-362

16. Tsuboi N, Kodama I, Toyama J, Yamada K: Anisotropic conduction properties of canine ventricular muscles- influence of high extracellular $\mathrm{K}^{+}$concentration and stimulation frequency. Jpn Circ $\mathrm{J}$ $1985 ; 49: 487-498$

17. Kléber A, Janse M, Wilms-schopmann F, Wilde de A, Coronel R. Changes in conduction velocity during acute ischeamia in ventricular myocardium of the isolated porcine heart. Circulation 1986;73:189-198

18. Osaka T, Kodama I, Tsuboi N, Toyama J, Yamada K: Effects of activation sequence and anisotropic cellular geometry on the repolarization phase of action potential of dog ventricular muscles. Circulation 1987;76:226-236

19. Spach M, Dolber P, Heidlage F. Interaction of inhomogeneities of repolarization with anisotropic propagation in dog atria. A mechanism for both preventing and initiating reentry. Circ Res 1989;65:1612-1631

20. Loewenstein W. Permeability of membrane junctions. Ann NY Acad Sci 1966;137:441-472 


\section{CHAPTER 7}

\section{MAPPING OF RESET OF FUNCTIONAL AND ANATOMIC REENTRY IN ANISOTROPIC VENTRICULAR MYOCARDIUM.}

Lucas Boersma

Josep Brugada Charles Kirchhof

Maurits Allessie

Circulation 1994;89:(in press) 


\section{INTRODUCTION}

The presence of a less than compensatory pause after a premature beat indicates reset of a cardiac rhythm and has been described for both normal and abnormal automaticity $^{1,2}$ as well as for reentry..$^{3-6}$ The response to premature stimulation has gained special importance in the treatment of ventricular tachycardia (VT) after myocardial infarction, ${ }^{7,8}$ which is most likely due to reentry. ${ }^{9-11}$ In a number of studies premature stimulation has provided evidence that during clinical VT a partially or fully excitable gap exists in the circuit. ${ }^{3-6}$ The response of VT to pre-mature stimulation is also used to evaluate the effects of anti-arrhythmic drugs on the reentrant circuit. ${ }^{12-14}$

Although the reset response may reveal some of the conduction properties within and around the reentrant circuit, ${ }^{5}$ it has not yet been determined whether clinical ventricular tachycardia is based on reentry around a functional or an anatomic barrier. Many studies have shown that the reentrant circuit after myocardial infarction comprises an area of slow conduction ${ }^{15-20}$ and that such a segment of slow conduction influences the reset response. $6,21,22$

All these electrophysiologic studies have greatly contributed to our understanding of reset of reentrant tachycardias. However, so far mapping of the activation patterns of the reentrant circuit during reset has only been performed in a limited number of studies, ${ }^{2324}$ and direct evidence of the events taking place during reset is still lacking. The goal of the present investigation was to study the response to premature stimulation by high resolution mapping in two experimental models of sustained VT in Langendorff perfused rabbit hearts. In one model, reentry occurred around a fixed anatomical obstacle, while in the other model no gross anatomical obstacle was present and reentry occurred around a line of functional conduction block. The specific goals of our study were: a) to determine the width and nature of the excitable gap, b) to examine whether the different types of reentry could be charac-terized by a typical reset response, and c) to study the role of an area of slow conduction in the reentrant circuit in the reset response.

\section{METHODS}

\section{Preparation}

In this study, in 18 Langendorff-perfused rabbit hearts a thin layer of left ventricular subepicardium was created by the cryoprocedure. The perfusion system and the cryoprocedure have been discussed in chapter 2 .

\section{Recording and Stimulation}

A spoon-shaped mapping electrode, molded to the epicardial surface of the left ventricle was used, containing 248 silver electrodes (diameter $0.3 \mathrm{~mm}$, interelectrode spacing $2.25 \mathrm{~mm}$ ). The on-line mapping system to record and analyze the data has been described in chapter 3 . In the activation maps conduction block was defined as a delay in conduction of more than $20 \mathrm{~ms}$ between adjacent recording sites (apparent conduction velocity $<10 \mathrm{~cm} / \mathrm{s}$ ). In addition the tissue distal to the site of block should be activated from a different direction.

A computer-controlled stimulator (Medtronic SP3084) was used for bipolar stimulation through any pair of electrodes selected from the mapping electrode. Sustained 
monomorphic ventricular tachycardia was induced by incremental pacing. During sustained anatomic VT premature stimuli were applied at 4 times diastolic threshold at 8 different sites around the ring. During functional VT premature stimulation (4 times diastolic threshold) was performed at 2 different pacing sites located either at a pivot point of the circuit or halfway along the central line of block.

An electrogram recorded adjacent to the pacing site was used to synchronize the stimulator. The last local activation preceding the stimulus was defined as V1. The first two activations following the stimulus were defined as V2 and V3. The coupling interval between activation of the reference electrogram (V1) and the stimulus (S) was shortened in steps of $2 \mathrm{~ms}$. After the experiment the exact coupling interval at the site of pacing was determined by interpolation of all actual V1-S intervals around the pacing site. The effective refractory period (ERP) was deter-mined as the longest V1-S interval that failed to initiate a propagated response. The FRP was determined as the shortest V1-V2 interval at the nearest electrode orto-dromic to the site of pacing. The difference between the cycle length of VT and the ERP was defined as the excitable gap (EG).

During VT, reset curves of several recording sites were obtained by premature stimulation at decreasing coupling intervals (V1-S). Two different curves were constructed. In the first curve the interval was plotted between the last activation during VT and the first activation after the stimulus (V1-V2). The second curve plotted the interval between the first and second (V2-V3) activation after the stimulus. At the pacing site the V2-V3 interval represents the return cycle of the premature stimulus.,4

\section{Statistical Analysis}

Results were compared using the paired Student's t-test, Analysis of Variance or Bonferroni's t-test, and linear regression when appropriate. P-values of less than 0.05 were taken as statistically significant.

\section{RESULTS}

\section{Characteristics of Reentry around an Anatomical Obstacle}

In 10 experiments rapid pacing induced reentrant ventricular tachycardia (VT) around a central anatomic obstacle. In 6 hearts anatomic VT was based on a clockwise and in 4 hearts on a counterclockwise circulating wave. The average cycle length of anatomic VT was $167 \pm 17 \mathrm{~ms}$ (range 148-192 ms) (Table 7.1). The cycle length of VT was mainly determined by the length of the segment of slow conduc-tion between the LAD and the central obstacle where the impulse propagated transverse to the epicardial fiber orientation. During fast VTs this segment of slow conduction was shorter than during slow VTs. All VTs were longlasting and stable with a beat-to-beat variation in cycle length of less than $2 \mathrm{~ms}$. Spontaneous termina-tion of VT was never observed.An example of an anatomic VT is given in figure 7.1. The upper left map shows a clockwise circulating wave around a central obstacle, with a revolution time of $169 \mathrm{~ms}$. In the corridor between the LAD and the obstacle, between the 20 and the $90 \mathrm{~ms}$ isochrone, the impulse propagated slowly $(23 \mathrm{~cm} / \mathrm{s})$ transverse to the epicardial fiber orientation. At the base and the free wall impulse propagation was parallel to the fiber axis and proceeded at an average speed of $78 \mathrm{~cm} / \mathrm{s}$. The lower left map shows the distribution of the effective refractory periods (ERP) during this VT. In this example the refractory 


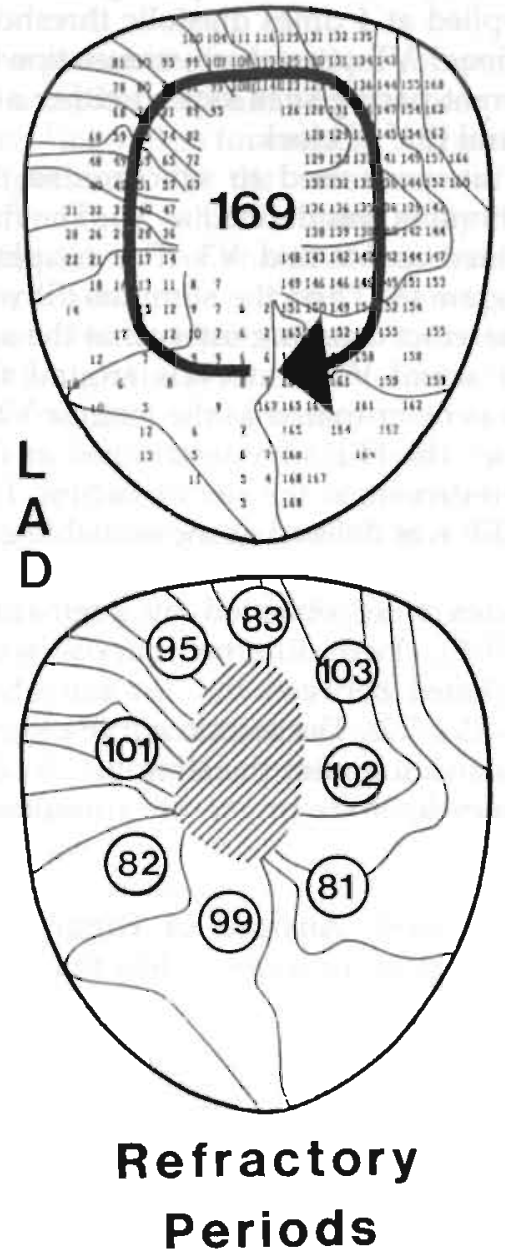

\section{Reset of Anatomic VT}
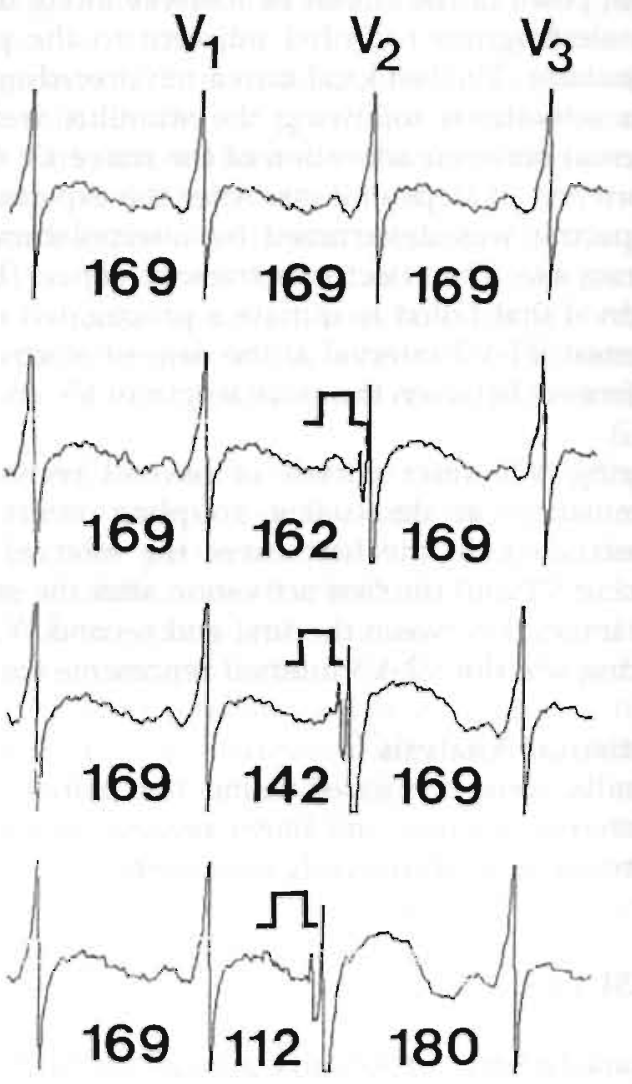

Figure 7.1: Left: Activation map showing a clockwise VT around a central anatomical obstacle with a cycle length of $169 \mathrm{~ms}$. In the corridor between the LAD and the central obstacle conduction was slow due to propagation transverse to the epicardial fiber orientation (crowding of isochrones). The lower panel shows the refractory periods during VT at 8 sites around the circuit. Activation times and refractory periods are given in milliseconds. Isochrones are drawn at $10 \mathrm{~ms}$ intervals. The arrow indicates the direction of the circulating wave. LAD: Left Anterior Descending artery. Right: Reset of anatomic VT by single premature stimuli with coupling intervals of 150,130, and $100 \mathrm{~ms}$ which shortened the V1-V2 intervals to 162, 142, and $112 \mathrm{~ms}$ respectively. Only after the earliest premature beat (bottom tracing) the V2-V3 interval was slightly prolonged due to slowing of conduction of the premature depolarization wave.

periods around the ring varied between 81 and $103 \mathrm{~ms}$ (average $93 \pm 10 \mathrm{~ms}$ ). Thus a large excitable gap existed in the circuit, varying between 66 and 88 ms (average $76 \pm 10$ $\mathrm{ms})$. 
Table 7.1:

Characteristics of Anatomic Ventricular Tachycardia.

\begin{tabular}{ccccccc}
\hline EXP & CL & ERP & EG & V1-V2 & V2-V3 & $\begin{array}{c}\text { MAX } \\
\text { RESET } \\
(\mathrm{ms})\end{array}$ \\
\hline 1. & 148 & $86 \pm 5$ & $62 \pm 5$ & $102 \pm 5$ & $159 \pm 3$ & $35 \pm 3$ \\
2. & 151 & $76 \pm 4$ & $75 \pm 4$ & $94 \pm 6$ & $156 \pm 2$ & $52 \pm 2$ \\
3. & 151 & $93 \pm 6$ & $58 \pm 9$ & $109 \pm 6$ & $161 \pm 6$ & $33 \pm 4$ \\
4. & 154 & $103 \pm 9$ & $51 \pm 9$ & $116 \pm 6$ & $161 \pm 7$ & $30 \pm 4$ \\
5. & 158 & $88 \pm 11$ & $70 \pm 11$ & $103 \pm 9$ & $170 \pm 6$ & $44 \pm 4$ \\
6. & 166 & $99 \pm 12$ & $67 \pm 12$ & $115 \pm 11$ & $180 \pm 9$ & $38 \pm 5$ \\
7. & 169 & $93 \pm 10$ & $76 \pm 10$ & $113 \pm 8$ & $189 \pm 13$ & $36 \pm 6$ \\
8. & 187 & $103 \pm 11$ & $84 \pm 11$ & $123 \pm 8$ & $196 \pm 7$ & $54 \pm 2$ \\
9. & 191 & $93 \pm 13$ & $98 \pm 13$ & $113 \pm 11$ & $202 \pm 9$ & $68 \pm 7$ \\
10. & 192 & $114 \pm 17$ & $78 \pm 17$ & $134 \pm 8$ & $201 \pm 5$ & $49 \pm 9$ \\
\hline AVG & 167 & 95 & 72 & 112 & 178 & 44 \\
SD & 17 & 11 & 14 & 11 & 18 & 12 \\
\hline
\end{tabular}

$E X P=$ experiment, $C L=$ cycle length, $E R P=$ effective refractory period, $E G=$ excitable gap $V 1-V 2=$ interval between last activation before and first activation after stimulation, V2-V3 = interval between first and second actioation after stimulation, Max Reset $=$ maximal reset, $A V G=$ average, $S D=$ standard deviation

Comparison of the local ERPs in all 10 hearts did not reveal a certain area where the ERP was systematically different from the other sites (Bonferroni's t-test, $p>0.05$ ). Due to the large excitable gap anatomic VT could be easily reset by single premature stimuli. In the right panel of figure 7.1 the responses to various premature stimuli are shown. With increasing prematurity, the V1-V2 interval was shortened to 162,142 , and $112 \mathrm{~ms}$ respectively and VT was reset. In the lowest tracing the V1-V2 interval of $112 \mathrm{~ms}$ was followed by a prolongation of V2-V3 from 165 to $180 \mathrm{~ms}$ due to a slight slowing of conduction of the premature wavefront.

In table 7.1 the cycle length, effective refractory period (ERP), and excitable gap (EG) are given for all 10 hearts. The ERP and the EG are given as average values of 8 pacing sites in each heart. In the table the VTs are arranged according to their cycle length. The average ERP was $95 \pm 11 \mathrm{~ms}$, resulting in an EG of $72 \pm 14 \mathrm{~ms}$. Linear regression showed that during slower VTs the ERP tended to be longer (slope $0.38, \mathrm{R}=0.63, \mathrm{P}=0.051$ ) and the EG was larger (slope $0.62, \mathrm{R}=0.78, \mathrm{p}=0.006$ ). Due to latency between the stimulus and the response the functional refractory period adjacent to the pacing site was 112 $\pm 11 \mathrm{~ms}$. At the shortest coupling intervals the return cycle was slightly prolonged (178 
Reset of Anatomic VT
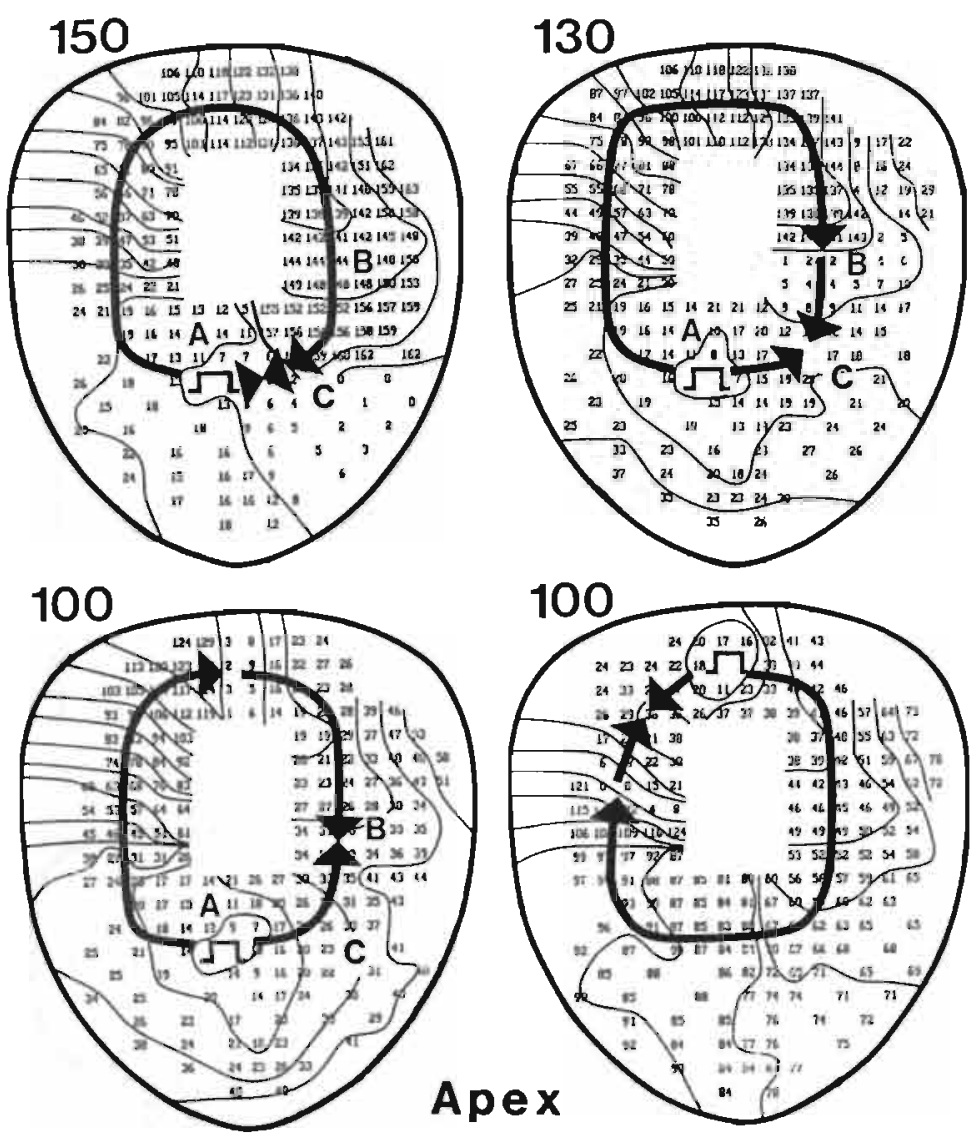
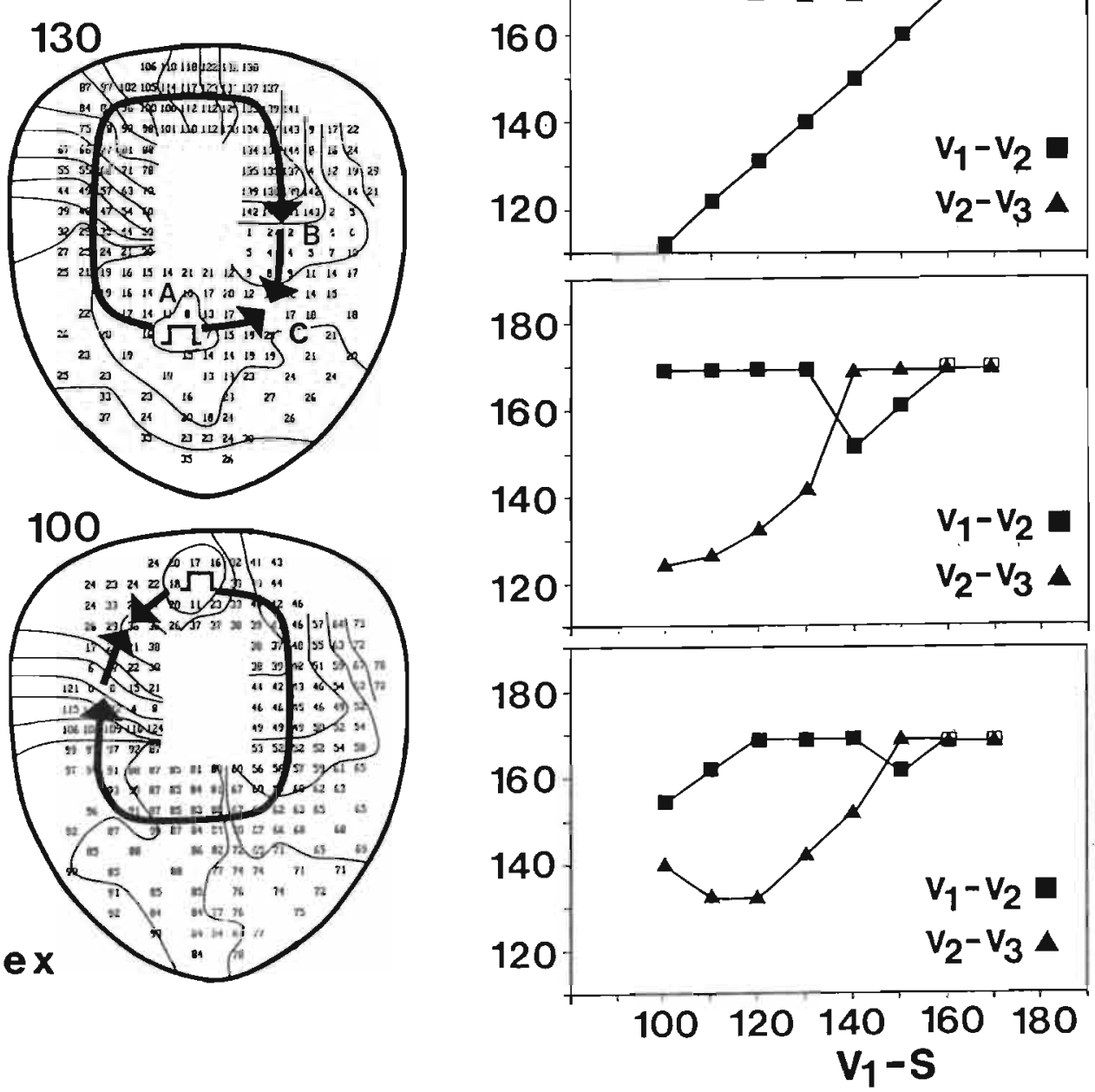
$\pm 18 \mathrm{~ms}$ ) compared to the cycle length of VT $(167 \pm 17 \mathrm{~ms})$, due to slowing of conduction of the premature wavefront. The average maximal amount of reset of anatomic VT was $44 \pm 12 \mathrm{~ms}$. Anatomic VT was never terminated by single premature stimuli, indicating that all segments of the ring had a high safety factor for conduction.

\section{Mapping of Reset of Anatomic VT by Premature Stimuli}

In all 10 hearts anatomic VT could be reset from all 8 pacing sites that were tested. Figure 7.2 gives the activation maps during reset of a clockwise VT (cycle length 169 $\mathrm{ms}$ ) both from a pacing site proximal and distal to the segment of slow conduction. As can be seen from the upper left map, a late premature beat (V1-S=150 ms) induced proximal to the segment of slow conduction reset the circuit in orthodromic direction, while the paced antidromic wave collided with the clockwise circulating wave. Due to the minimal degree of prematurity, only $6 \%$ of the circuit was antidromically invaded by the paced impulse. During reset of VT with shorter coupling intervals the paced antidromic wave invaded a progressively larger part of the circuit before colliding with the circulating wave. In the upper right map (V1-S=130 ms) and the lower left map (V1$\mathrm{S}=100 \mathrm{~ms}$ ) the circuit was antidromically invaded for about $11 \%$ and $25 \%$ respectively. Apart from the degree of prematurity, the amount of antidromic invasion of the circuit wasalso determined by the localiza-tion of the pacing site relative to the segment of slow conduction. This can be seen in the lower right map, in which the pacing site was located distal to the segment of slow conduction. In such a case the antidromic wave penetrated a much smaller part of the circuit, and even a very early premature stimulus (V1-S=100 ms) invaded only $9 \%$ of the circuit antidromically.

During the premature beat the circuit was divided into a paced orthodromic segment (ORTHO), a paced antidromic segment (ANTI), and a segment in which the circulating wave was still present (ORTHO-1). At progressively premature stimuli the ORTHO segment became smaller while the ANTI and ORTHO-1 segment occupied a larger portion of the circuit. Consequently, at different degrees of prematurity a given site in the reentrant circuit could be located in different segments. If such a change from one to another segment occurred the reset curve obtained at that given site suddenly changed. This is shown in the right part of figure 7.2 where the reset curves are given for three different recording sites in the circuit. The top graph shows the typical reset curve for a recording site that is always located in the ORTHO segment. A progressive decrease in V1-V2 interval is associated with a flat V2-V3 curve, indicating reset of VT. Only at the shortest coupling intervals the return cycle (V2-V3) slightly increases due to slowing of conduction of the prema-ture wavefront. The middle graph shows the

Figure 7.2 (left): Left: Four activation maps during reset of the anatomic VT shown in figure 7.1 by single. premature stimuli with a coupling interoal of 150, 130, and $100 \mathrm{~ms}$. In the first three maps the pacing site was located proximal to the segment of slow conduction. The lower right panel shows activation map during maximal reset $(V 1-S=100 \mathrm{~ms}$ ) at pacing site distal to the segment of slow conduction. During each premature stimulus the paced antidromic waze (ANTI) collided with the ongoing circulating wave (ORTHO-1), while the paced orthodromic wave (ORTHO) reset VT. The moment of stimulation was taken as $t=0$. Actizution times are given in milliseconds and isochrone lines are drawn at $10 \mathrm{~ms}$ intervals. Arrows indicate the different wave fronts. Right: Three different reset curves of anatomic VT. Pacing was performed proximal to the site of stimulation. The three curves were obtained from recording sites $A, B$, and $C$, as indicated in the maps. The coupling interval of the premature stimulus (V1-S) was plotted on the abscissa. V1-V2 (squares) and V2-V3 (triangles) intervals were plotted on the ordinate. See text for discussion. 
typical reset curve for a recording site which during late premature stimuli is located in the ORTHO segment but which during earlier premature stimuli becomes part of the ORTHO-1 segment. During late premature stimuli V1-V2 gradually decreases with a flat V2-V3 curve. During earlier premature stimuli however, the V1-V2 curve becomes flat and now the V2-V3 intervals shorten. The bottom panel shows the reset curve recorded in the area antidromic to the pacing site. During late premature stimuli here the reset curve is similar as in the ORTHO segment. During more premature stimuli however the recording site first becomes part of the ORTHO-1 segment and during very early premature beats (coupling intervals $<120 \mathrm{~ms}$ ) becomes located in the ANTI segment. As a result, at coupling intervals $<120 \mathrm{~ms}$ the V1-V2 interval progressively shortens again while the V2-V3 interval either remains constant or prolongs a little, due to slowing of conduction of the early premature paced orthodromic wave. These three typical reset responses thus reveal the relative position of the pacing and recording sites within the reentrant circuit. A reset response as in the upper panel of figure 7.2 identifies a recording site close to and orthodromic from the pacing site. The middle graph indicates a more distinct orthodromic recording site, while a reset response like in the bottom panel was exclusively found in the antidromic segment.

During pacing proximal to the area of slow conduction the three different reset curves were all observed in fairly large parts of the circuit. However, if the pacing site was distal to the segment of slow conduction the ANTI and ORTHO-1 segments were both very small (see right lower reset map). Consequently most reset curves were of the ORTHO type because the other response types remained concealed in the segment of slow conduction.

\section{Characteristics of Reentry around a Line of Functional Block}

In the absence of a gross anatomical obstacle in the thin epicardial layer, slow pacing resulted in uniform anisotropic conduction. In the left panel of figure 7.3 the activation map of the intact layer of epicardium during pacing at $500 \mathrm{~ms}$ intervals is given. From the site of stimulation the impulse propagated radially with ellipsoid shaped isochrones. The long axis of the ellipse represents fast conduction parallel to the epicardial fiber orientation ( 66 and $71 \mathrm{~cm} / \mathrm{s}$ ), while along the short axis the impulse propagates perpendicular to the fiber direction $(27$ and $17 \mathrm{~cm} / \mathrm{s}){ }^{25}$ During rapid pacing at intervals shorter than 120 ms local arcs of conduction block developed. ${ }^{26}$ In 8 of 18 hearts, stable reentrant VT around one of these arcs of functional conduction block was induced. The activation map in the middle of figure 7.3 gives an example. During functional VT (cycle length $120 \mathrm{~ms}$ ) the central line of block was oriented parallel to the fiber direction and the reentrant circuit had an oval shape. Propagation along both long limbs of the circuit was fast $(40$ and $73 \mathrm{~cm} / \mathrm{s})$. At the pivoting points the circulating wave made a sharp U-turn transverse to the fiber direction, and the conduction velocity became as slow as $15 \mathrm{~cm} / \mathrm{s}$. The electrograms at the right show the continuous circulation of the impulse around the line of functional block in the direction from electrodes $\mathrm{A}$ to $\mathrm{L}$. Electrograms C-D and $\mathrm{H}-\mathrm{J}$ recorded at the pivoting points of the circuit exhibited low amplitude and fragmen-ted potentials, while the electrograms from the longitudinal limbs of the circuit were of high amplitude. In 7 of 8 hearts functional VT was based on reentry around a single line of block while in one heart figure-of-eight reentry occurred.

The cycle length of the 8 functional VTs ranged between 94 and $121 \mathrm{~ms}$ with an average of $110 \pm 10 \mathrm{~ms}$ (Table 7.2). All VTs were stable with a beat-to-beat variation of less than 


\section{Anisotropic Conduction Anisotropic Reentry}

\section{Cycle Length $500 \mathrm{~ms}$}

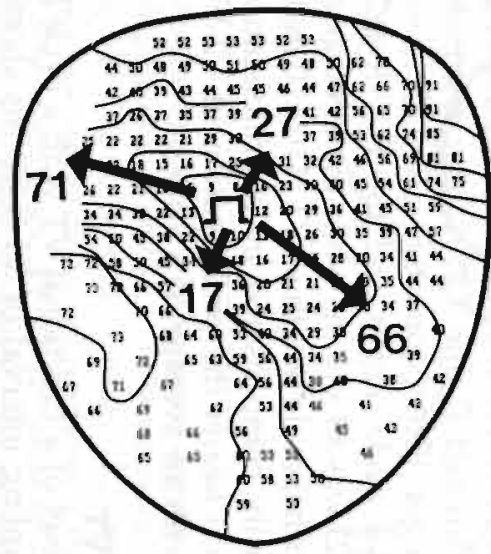

\section{Cycle Length $120 \mathrm{~ms}$}

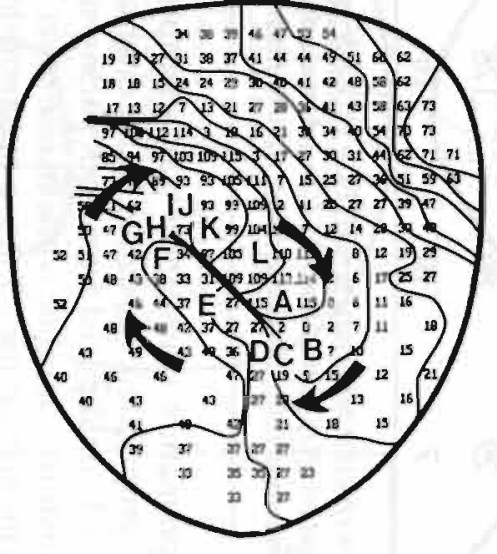

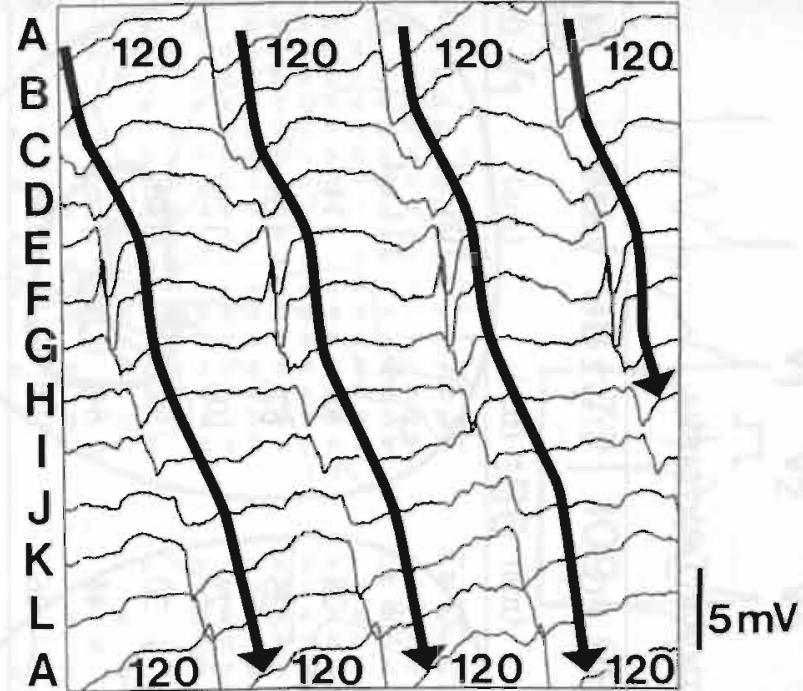

Figure 7.3: Left: Activation maps during slow pacing ( $S 1-S 1=500 \mathrm{~ms}$ ) and sustained VT (cycle length $120 \mathrm{~ms}$ ). During slow pacing the ellipsoid isochrones display the anisotropic conduction properties of the epicardium, with conduction parallel to the epicardial fibers (long arrows) being faster than in the transverse direction (short arrows). The conduction velocities in the four different directions are indicated on the map (in cm/s). The moment of stimulation was taken as $t=0$. During anisotropic reentry (right map) the line of block was oriented parallel to the fiber direction. In both long limbs of the circuit the conduction velocity was faster compared to transverse conduction at the pivoting points. The arronv indicates the direction of the circulating wave. Activation times are given in milliseconds and isochrone lines are drawn at $10 \mathrm{~ms}$ intervals. Right: Twelve electrograms (A-L) recorded along the functional anisotropic circuit. At the pivoting points the electrograms were of low amplitude whereas along both long limbs of the circuit sharp deflections of high amplitude were recorded. Calibration bar $=5$ milliVolts. 
Table 7.2:

Characteristics of Functional Ventricular Tachycardia.

\begin{tabular}{|c|c|c|c|c|c|c|}
\hline EXP & $\begin{array}{l}\mathrm{CL} \\
(\mathrm{ms})\end{array}$ & $\begin{array}{l}\text { ERP } \\
(\mathrm{ms})\end{array}$ & $\begin{array}{l}\text { EG } \\
(\mathrm{ms}) \\
\end{array}$ & $\begin{array}{c}\mathrm{V} 1-\mathrm{V} 2 \\
(\mathrm{~ms})\end{array}$ & $\begin{array}{c}\mathrm{V} 2-\mathrm{V} 3 \\
(\mathrm{~ms})\end{array}$ & $\begin{array}{c}\text { MAX } \\
\text { RESET } \\
(\mathrm{ms})\end{array}$ \\
\hline 1. & 95 & $68 \pm 3$ & $27 \pm 3$ & $72 \pm 5$ & $108 \pm 4$ & $10 \pm 2$ \\
\hline 2. & 98 & $70 \pm 2$ & $28 \pm 3$ & $77 \pm 4$ & $114 \pm 4$ & $6 \pm 8$ \\
\hline 3. & 107 & 79 & 28 & 88 & 114 & - \\
\hline 4. & 109 & 74 & 35 & 87 & 126 & - \\
\hline 5. & 114 & 93 & 21 & 100 & 128 & - \\
\hline 6. & 120 & 90 & 30 & 98 & 140 & - \\
\hline 7. & 120 & 79 & 41 & 84 & 156 & - \\
\hline 8. & 121 & 95 & 26 & 100 & 136 & 8 \\
\hline AVG & 110 & 81 & 29 & 89 & 128 & \\
\hline $\mathrm{SD}$ & 10 & 10 & 7 & 10 & 16 & \\
\hline
\end{tabular}

$E X P=$ experiment, $C L=$ cycle length, $E R P=$ effective refractory period, $E G=$ excitable gap, $V 1-V 2=$ interval between last activation before and first activation after stimulation, V2-V3=interval between first and second activation after stimulation, Max Reset = maximal reset; $A V G=$ average, $S D=$ standard deviation.

2 ms. Reset of functional VT by premature stimulation was attempted at 2 different pacing sites. In 6 of 8 hearts early propagated responses could only be elicited at one of the two pacing sites. In 2 hearts VT could paced from both pacing sites. In one heart VT was terminated by a single premature stimulus, while in one other heart premature stimulation transformed sustained monomorphic VT into a polymorphic non-sustained VT. The average ERP during VT was $81 \pm 10 \mathrm{~ms}$ and thus in all VTs a small excitable gap of $30 \pm 7 \mathrm{~ms}$ could be demonstrated (Table 7.2). Linear regression showed that slower VTs had a somewhat longer ERP (slope $0.86, \mathrm{R}=0.83, \mathrm{p}=0.011$ ), while no correlation was found between VT cycle length and the EG $(R=0.24, p=0.57)$. The functional refractory period (V1-V2) was $89 \pm 10 \mathrm{~ms}$ while the average return cycle (V2V3) of $128 \pm 16 \mathrm{~ms}$ exceeded the normal cycle length of VT (110 $\pm 10 \mathrm{~ms})$. In 5 of 8 hearts the shorter V1-V2 interval was fully compensated by a prolongation of the V2-V3 interval and VT was not reset. In 3 hearts the prolongation of V2-V3 was less than compensatory and VT was reset with an average of $8 \pm 2 \mathrm{~ms}$.

\section{Mapping of Reset of Functional VT}

Figure 7.4 shows an example of reset of a functional VT with a cycle length of $98 \mathrm{~ms}$. A premature stimulus with a coupling interval of $\mathrm{V} 1-\mathrm{S}=70 \mathrm{~ms}$ shortened the V1-V2 interval to $77 \mathrm{~ms}$ (top tracing). Despite an increase of V2-V3 to $109 \mathrm{~ms}$, the sum of the 

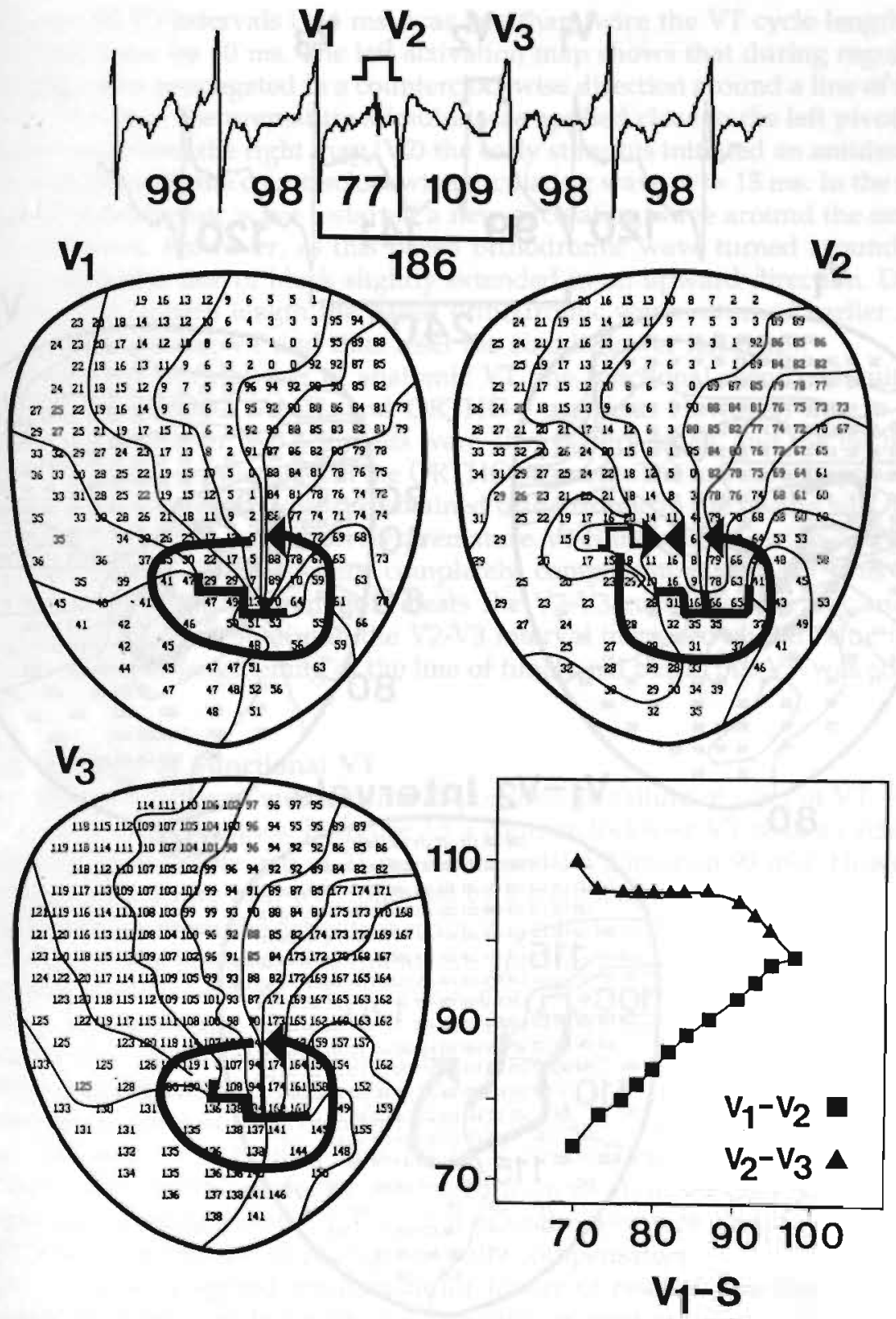

Figure 7.4 (right): Reset of functional VT by premature stimulation. A premature stimulus shortened V1-V2 to $77 \mathrm{~ms}$ with a slight prolongation of V2-V3 to $109 \mathrm{~ms}$, indicating reset of VT by $10 \mathrm{~ms}$. Three maps show the actioation during VT (V1), the premature stimulus (V2), and the first VT beat after the stimulus (V3). The premature stimulus was applied close to left pioot point of the circuit. Lower right panel showos complete reset curve obtained close to pacing site. V1-S is plotted on the abscissa, while V1-V2 (squares) and V2-V3 (triangles) are plotted on the ordinate. Activation times are given in milliseconds. Isochrones are draun at $10 \mathrm{~ms}$ intervals. Arrows indicate the several waves. During V2 the moment of stimulation was taken as $t=0$. 


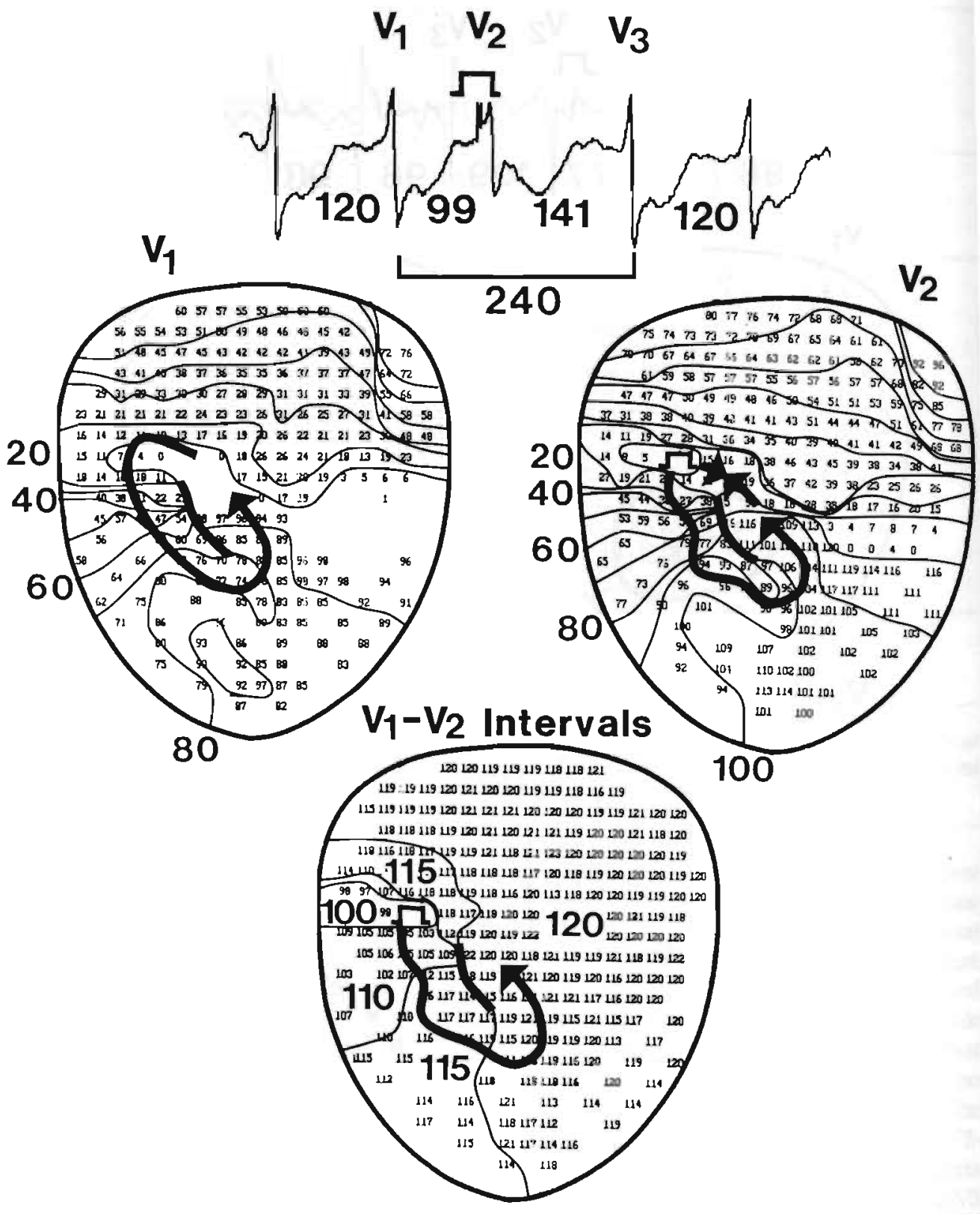

Figure 7.5: Failure of reset of functional VT by premature stimulation. During VT (cycle length $120 \mathrm{~ms}$ ) a premature stimulus shortened V1-V2 to $99 \mathrm{~ms}$ while V2-V3 prolonged to $141 \mathrm{~ms}$ (no reset). Left map (V1) shows the last $90 \mathrm{~ms}$ before premature stimulus. V2 shows actioation map after the premature stimulus $(t=0)$. Bottom panel shows V1-V2 interoal map. The premature stimulus was applied at left pivoting point of the circuit. Conduction zelocity of the paced orthodromic wave was depressed compared to that during VT (curoed arrow V2 map). Activation times are given in milliseconds and isochrones are drawn at $10 \mathrm{~ms}$ intervals. Arrows indicate the several waves. See text for description. 
V1-V2 and V2-V3 intervals (186 ms) was less than twice the VT cycle length (196 ms) and VT was reset by $10 \mathrm{~ms}$. The left activation map shows that during regular VT the circulating wave propagated in a counterclockwise direction around a line of functional block. In this case, the premature stimulus was applied close to the left pivoting point. As can be seen from the right map (V2) the early stimulus initiated an antidromic wave which collided with the counterclockwise circulating wave at $\mathrm{t}=15 \mathrm{~ms}$. In the meantime the paced orthodromic wave restarted a new circulating wave around the same line of functional block. However, as this paced orthodromic wave turned around the right pivoting point the line of block slightly extended in an upward direction. Despite the slight increase in path length the paced orthodromic wave returned earlier at the site of pacing than during VT and thus reset the circuit (lower left map).

As was the case during reset of anatomic VT, the functional reentry circuit was also divided in an ORTHO, ANTI, and ORTHO-1 segment. However, due to the small excitable gap the latter two segments were always very small, and the far majority of the recording sites was located in the ORTHO segment. The lower right panel of figure 7.4 shows the reset response curve obtained orthodromic to the pacing site. The V1-V2 intervals shortened at progressively premature V1-S intervals. During late premature stimuli the V2-V3 intervals were completely compensatory and VT was not reset. However, during earlier premature beats the V2-V3 curve became flat and VT was reset. During the earliest stimuli the V2-V3 interval increased slightly due to slowing of conduction and lengthening of the line of functional block, but VT was still reset by $10 \mathrm{~ms}$.

\section{Failure of Reset of Functional VT}

There were two different mechanisms responsible for failure of reset of V' $\Gamma$. Figure's 7.5 and 7.6 show two examples. In figure 7.5 a counterclockwise VT with a cycle length of $120 \mathrm{~ms}$ had an excitable gap of $21 \mathrm{~ms}$ (shortest V1-V2 interval $99 \mathrm{~ms}$ ). However, even this earliest premature beat was followed by a completely compensa-tory pause of 141 ms (top tracing). From the activation maps it can be seen that, as in figure 7.4 in which VT was reset, the stimulus did initiate an antidromic wave which collided with the circulating wave at $\mathrm{t}=15 \mathrm{~ms}$. The stimulus also started a new orthodromic wave which restarted the reentrant VT. However, in this heart the conduction velocity of the premature orthodromic wave was markedly depressed, as indicated by the crowding of isochrones between $\mathrm{t}=20$ and $\mathrm{t}=100 \mathrm{~ms}$ in map V2. As a consequence the premature impulse gradually lost its prematurity while propaga-ting along the lower limb of the circuit. The map at the bottom gives the local intervals between V1 and V2 and shows that the V1-V2 interval gradually prolonged from $99 \mathrm{~ms}$ at the pacing site to $120 \mathrm{~ms}$ at the opposite pivoting point. Thus, only part of the reentrant circuit was reset and the V2-V3 interval at the site of pacing was fully compensatory.

Figure 7.6 shows a second mechanism for failure of reset of functional VT. During a functional VT with a cycle length of $120 \mathrm{~ms}$ the shortest coupling interval that elicited a propagated response was $79 \mathrm{~ms}$. Again, the resulting shortening of the V1-V2 interval ( $84 \mathrm{~ms}$ ) however was fully compensated by prolongation of the return cycle (V2-V3=156 $\mathrm{ms})$. During VT (left activation map) the impulse circulated in a clockwise direction around a line of functional block. The premature stimulus was applied at the apex which was part of the lower limb of the circuit. At first sight, the antidromic wave seems to collide with the circulating wave while the paced orthodromic wave restarts a new VT around the same line of block (V2). However, the V1-V2 interval map at the 


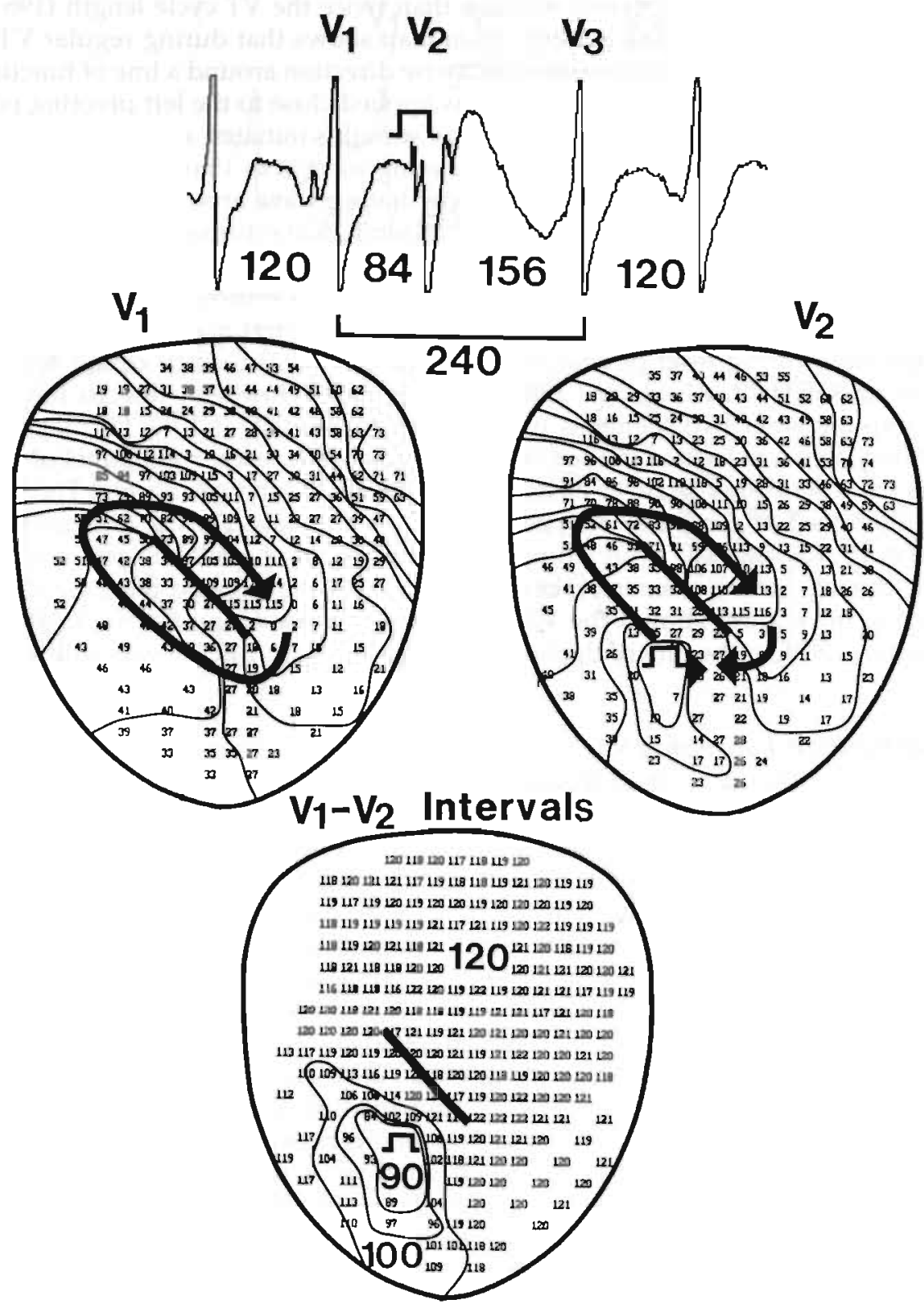

Figure 7.6: Failure of reset of functional VT (cycle length $120 \mathrm{~ms}$ ) by premature stimulation in a different heart as in figure 7.5. The earliest premature stimulus shortenened V1-V2 to $84 \mathrm{~ms}$ but V2-V3 was fully compensatory (156 ms) and VT was not reset. Two maps below the electrogram show activation maps during clockwise VT (V1) and during application of the premature stimulus $(t=0)(V 2)$. Bottom panel shows interval map of V1-V2 intervals. Premature stimulus was applied at apex close to lower limb of the circuit. Activation times are given in milliseconds. Isochrones are drawn at $10 \mathrm{~ms}$ intervals. Arrows indicate the several waves. 
bottom shows that this was actually not the case. Although around the pacing site the local intervals were shortened to less than $90 \mathrm{~ms}$, the V1-V2 intervals at the lower limb of the reentrant circuit along the line of block remained $120 \mathrm{~ms}$. The premature stimulus thus failed to penetrate the reentrant circuit, despite the fact that the pacing site was very close to the central line of block. This can be explained by the anisotropic properties of the ventricular myocardium. In the lower limb of the reentrant pathway conduction was fast because it was longitudinal to the epicardial fiber axis. In contrast, in order to reach the line of block the paced premature wave had to propagate transverse to the fiber orientation. The resulting slow conduction prevented the premature wave to short-circuit the circulating wave despite the fact that the pacing site was close to the center of the circuit, and as a result VT was not reset.

\section{DISCUSSION}

\section{Premature Stimulation during Reentrant VT}

A large number of experimental and clinical studies have shown that in the chronic phase after myocardial infarction, VT is based on reentrant excitation..$^{9,10,11,15-20}$ Reset of VT by premature stimulation has been used to characterize the conduction properties of the reentrant pathway. In clinical studies, a reset window of $43 \%$ of the VT cycle length was found. ${ }^{3-6}$ The reset curve usually has a flat part which is taken as evidence for an anatomic reentrant circuit with a fully excitable gap. The prolon-gation of the return cycle has been explained by decremental conduction either within the reentrant pathway itself or in the tissue between the pacing site and the circuit. ${ }^{5}$ Several investigations have studied reset of reentrant VT in 4-6 days old canine myocardial infarction. ${ }^{15-17,24}$ In this experimental model, $\mathrm{VT}$ is due to reentry in the epicardial border zone of the infarct, either as a single circulating wave around a line of functional conduction block ${ }^{18}$ or as figure-of-eight reentry. ${ }^{15,2324}$ El-Sherif et al. ${ }^{23}$ showed that premature stimuli could reset figure-of-eight reentry, and that decremental conduction of early premature impulses within the central common pathway could terminate VT. In this study only a partially excitable gap was demonstrated. In the same experimental model the reset response was systematically investigated by Hanna et al. ${ }^{24}$ They found that late premature beats were conducted without a delay in the reentrant circuit, demonstrating the presence of a fully excitable gap.

In our study, the reset characteristics of functional and anatomic reentry in a thin epicardial layer of the left ventricle were compared. During anatomic reentry, all sites in the circuit had a refractory period that was markedly shorter than the conduction time around the obstacle, resulting in an excitable gap measuring $43 \%$ of the VT interval The reset curve was flat, indicating that during premature beats the excitability was fully recovered. Only during the earliest premature stimuli the return cycle slightly increased, due to partial recovery of excitability. Similar data were reported by Bernstein et al. ${ }^{27}$ in a canine model of ventricular reentry around the orifice of the mitral and aortic valves. The reset characteristics of these experi-mental models of anatomic VT thus are similar to the reset response of clinical VT. It should be noted that in our study, anatomic VT was always reset from a pacing site within the circuit. This may explain the larger window of reset in our model compared to clinical VTs, in which pacing from a site within the circuit is not always possible.

In contrast to our model of anatomic VT, the majority of functional VTs could usually 
not be reset, despite the presence of an excitable gap of $28 \%$ of the VT interval. Failure of reset was due to two different mechanisms:

1) Despite the fact that the pacing site was close to the circuit, slow anisotropic conduction prevented paced impulses from short-circuiting the circulating wave. Also in clinical VTs, a long conduction time from the pacing site to the circuit has been demonstrated to prevent reset of the tachycardia. ${ }^{5}$

2) In case the premature impulses did penetrate the circuit, reset of the whole circuit was prevented by conduction slowing of the premature wavefront within the circuitous pathway. Thus, although the premature impulse did interrupt the circulating wave, its prematurity was gradually lost. As a result, only part of the circuit was reset and the curtailed cycle was followed by a fully compensatory pause.

\section{The Nature of the Partially Excitable Gap in Anisotropic Reentry}

The nature of the excitable gap during anisotropic reentry is not well understood. ${ }^{26,28-30}$ Three possible mechanisms should be considered:

1) Micro-anatomical barriers at the pivoting points of the circuit may enlarge the central line of functionally determined block. This may also anchor the functional circuit to a fixed position in the ventricles.

2) Sudden changes in axial current load, due to branching of the myocardial fibers or a sudden change in direction of propagation, may lead to decremental conduction or conduction block despite the fact that the cells are readily excitable. ${ }^{31-33}$ Computer simulations have shown that a sharp curvature of a circulating wave greatly diminishes its stimulating efficacy and conduction velocity. ${ }^{30}$ During the sharp U-turn at the pivoting points of an anisotropic circuit, the circulating wave suddenly encounters a high axial current load of the well-coupled fibers in the longitudinal limb of the circuit. Such a mismatch between the amount of generated excitatory current and the high electrotonic load at the pivoting point may result in an initial failure to activate the longitudinal limb of the circuit and only after a certain delay, when a larger part of the circulating wave has rotated around the pivoting point, the impulse may succeed to complete its 180 degree turn. Such a delay in conduction at the pivoting points will result in prolongation of the cycle length of $\mathrm{VT}$, creating an excitable gap in the circuit. 3) During anisotropic reentry the considerable differences in activation time between the cells at opposite sides of the pivoting points may result in electrotonic prolon-gation of the action potential. ${ }^{34}$ The associated lengthening in refractory period at the pivoting points may create an excitable gap in all other parts of the circuit.

During reset of one of the functional VTs in the present study, the central line of functional block lengthened when the premature wavefront tried to make a sharp Uturn at one of the pivoting points. This observation indicates that at the pivoting points the safety factor for conduction is lower than in other parts of the circuit. The resulting conduction delay at the pivoting points will create a small excitable gap in the other parts of the circuit.

\section{Importance of the Localization of the Pacing and Recording Sites}

Rosenthal et al. ${ }^{6}$ found that in $55 \%$ of cases reset of clinical VT was accompanied by fusion beats in the surface ECG, indicating that the pacing site was located proximal to the area of slow conduction. ${ }^{35,36}$ Absence of changes in QRS-morphology during reset has been attributed to pacing distal to or within the area of slow conduction. ${ }^{21}$ In our study, the relative positions of the pacing and recording electrodes in the reentrant 
circuit were distinguished by three characteristic reset curves, the position of the pacing site relative to the segment of slow conduction determining the likelyhood of each of these reset curves. If the pacing site was proximal to the segment of slow conduction, reset curves with either a single or a double sudden change in the reset were seen in up to $50 \%$ of the recording sites. In contrast, when the pacing site was located distal to the segment of slow conduction, more than $85 \%$ of the recording sites showed a regular ORTHO reset curve.

In a clinical study on the reset response of VT, Kay et al. ${ }^{22}$ have postulated that during pacing proximal to the area of slow conduction the electrograms recorded distal to the area of slow conduction should show a conduction time that exceeded the VT cycle length. In our study this was never observed, indicating that in clinical VTs the area of slow conduction shows a higher degree of decremental conduction during premature beats than the area of slow anisotropic conduction in normal myocardium. This is also indicated by the observation that in clinical VTs at least part of the reset curve is increasing, ${ }^{22}$ while up to $45 \%$ of VTs can be terminated by premature stimuli. ${ }^{4}$ In our study, the return cycle during reset of anatomical VT was almost completely flat and VT could not be terminated by premature stimulation.

\section{Clinical Implications}

Our results show that functional VT in anisotropic tissue has a small partially excitable gap. However, despite the fact that the pacing site was very close to the circuit, most functional VTs could not be reset. This was due both to slowing of conduction of the premature wave in the reentrant circuit or failure to penetrate the circuit. This implies that if the excitable gap of functionally determined reentrant circuits in patients is also very small, there is almost no chance that these functional VTs can be reset, especially since pacing is usually performed outside the reentrant circuit. Several studies on the reset characteristics of clinical VT have indicated that a fully excitable gap exists in the circuit. ${ }^{3-5}$ The results of our study suggest that these clinical VTs are probably not based on a functionally determined reentrant circuit.

Ventricular reentrant circuits in patients are very complex and usually comprise an area of slow conduction, ${ }^{17-20,41-43}$ which is the target for ablative therapy of VT. We found that the localization of the pacing and recording sites relative to the area of slow conduction could be identified on the basis of three typical reset curves. Recognition of these reset curves may be usefull to localize the area of slow conduc-tion in reentrant circuits in patients. However, a major limitation of our study was that all the pacing sites were located within the reentrant circuit. Since in the clinical setting pacing is usually performed outside the circuit, the value of these three typical reset curves thus remains to be determined.

\section{CONDENSED ABSTRACT}

In Langendorff perfused rabbit hearts thin layers of anisotropic left ventricular myocardium were created by a cryoprocedure. Rapid pacing induced sustained monomorphic ventricular tachycardia (VT) either around a line of functional conduction block $(n=8)$ or around a fixed anatomical obstacle $(n=10)$. In both models of VT the response to premature stimulation was tested. Anatomic VT could always be reset over a window of $43 \%$ of the cycle length of VT. The reset curve was flat, indicating that a 
gap of fully excitable tissue was present at every site in the circuit. Three typical local reset responses were found that identified the spatial and temporal localization of a recording site in the reentrant circuit. In the majority of functional VTs premature stimuli could not reset VT, despite the presence of a partially excitable gap (27\% of the cycle length). Failure of the premature wave to short-circuit the circulating impulse and slowing of conduction of the premature wavefront fully compensated the advancement of the tachycardia. 


\section{REFERENCES}

1. Strauss H, Saroff A, Bigger T, Glardina E: Premature atrial stimulation as a key to understanding of sinoatrial conduction in man. Presentation of data and critical review of the literature.

Circulation 1973;67;86-93

2. Dangman $\mathrm{K}$, Hoffman $\mathrm{B}$ : The effects of single premature stimuli on automatic and triggered rhythms in isolated canine Purkinje fibers. Circulation 1985;71:813-822

3. Almendral J, Stamato N, Rosenthal M, Marchlinski F, Miller J, Josephson M: Resetting response: patterns during sustained ventricular tachycardia: relationship to the excitable gap. Circulation 1986a;74:722-730

4. Almendral J, Rosenthal M, Stamato N, Marchlinski F, Buxton A, Frame L, Miller J, Josephson M: Analysis of the resetting response in sustained uniform ventricular tachycardia: incidence and relation to termination. J Am Coll Cardiol 1986b;8:294-300

5. Stamato N, Rosenthal M, Almendral J, Josephson M: The resetting response of ventricular tachycardia to single and double extrastimuli: implications for the excitable gap. Am J Cardiol 1987;60:596-601

6. Rosenthal M, Stamato N, Almendral J, Gottlieb C, Josephson M: Resetting of ventricular tachycardia with electrocardiographic fusion: incidence and significance. Circulation 1988;77:581588

7. Fisher J, Kim S, Furman S, Matos J: Role of implantable pacemakers in control of recurrent ventricular tachycardia. Am J Cardiol 1982;49:194-206

8. Waldecker B, Brugada P, Zehender M, Stevenson W, Den Dulk K, Wellens HJJ: Modes of electrical termination of ventricular tachycardia: importance for the selection of implantable antitachycardia device. Am J Cardiol 1986;57:150-155

9. Wellens $\mathrm{H}$, Schuilenburg $\mathrm{R}$, Durrer $\mathrm{D}$ : Electrical stimulation of the heart in patients with ventricular tachycardia. Circulation 1972;46:216-226

10. Wellens HJJ: Value and limitations of programmed electrical stimulation of the heart in the study and treatment of tachycardias. Circulation 1978;57:845-853

11. Josephson M, Horowitz L, Farshidi, Kastor J: Recurrent sustained ventricular tachycardia: 1 . Mechanisms. Circulation 1987;57:431-439

12. Kay G, Epstein A, Plumb V: Preferential effect of procainamide on the reentrant circuit of ventricular tachycardia. J Am Coll Cardiol 1989;14:382-390

13. Stamato N, Frame L, Rosenthal M, Almendral J, Gottlieb C, Josephson M: Procainamide-induced slowing of ventricular tachycardia with insights from analysis of resetting response patterns. Am J Cardiol 1989;63:1455-1461

14. Callans D, Hook B, Josephson M: The mechanism of propafenone-induced slowing of ventricular tachycardia in man as defined by analysis of resetting response patterns. PACE 1991;14:2035-2041

15. El-Sherif N, Smith R, Evans K: Canine ventricular arrhyhmias in the late myocardial infarction period. 8. Epicardial mapping of reentrant circuits. Circ Res 1981;49:255-267

16. Mehra R, Zeiler R, Gough W, El-Sherif N: Reentrant ventricular arrhythmias in the late myocardial infarction period. 9. Electrophysiologic-Anatomic correlation of reentrant circuits. Circulation 1983;67:11-24

17. Wit A, Allessie M, Bonke F, Lammers W, Smeets J, Fenoglio J: Electrophysiologic mapping to determine the mechanism of experimental ventricular tachycardia initiated by premature impulses. Am J Cardiol 1982;49:166-185

18. Dillon S, Allessie M, Ursell P, Wit A: Influences of anisotropic tissue structure on reentrant circuits in the epicardial borderzone. Circ Res 1988;63:182-206

19. de Bakker J, van Capelle F, Janse M, Wilde A, Coronel R, Becker A, Dingemans K, van Hemell N, Hauer R: Reentry as a cause of ventricular tachycardia in patients with chronic ischemic heart disease. Electrophysiologic and anatomic correlation. Circulation 1988;77:589-606

20. de Bakker J, Coronel R, Tasseron S, Wilde A, Opthof T, Janse M, van Capelle F, Becker A, Jambroes G: Ventricular tachycardia in the infarcted, Langendorff-perfused human heart: Role of the arrangement of surviving cardiac fibers. J Am Coll Cardiol 1990;15:1594-1607

21. Stevenson W, Weiss J, Wiener I, Nademanee K, Wohlgelernter D, Yeatman L, Josephson M, Klitzner T: Resetting of ventricular tachycardia: implications for localizing the area of slow conduction. J Am Coll Cardiol 1988;11:522-529 
22. Kay G, Epstein A, Plumb V: Resetting of ventricular tachycardia by single extrastimuli. Relation to slow conduction within the reentrant circuit. Ciculation 1990;81:1507-1519

23. El-Sherif N, Gough W, Restivo M: Reentrant ventricular tachycardia in the late myocardial infarction period. 14. Mechanisms of resetting, entrainment, acceleration, or termination of reentrant tachycardia by programmed electrical stimulation. PACE 1987;10:341-371

24. Hanna M, Coromilas J, Wit A, Dillon S, Josephson M: Insights into the mechanisms of resetting of ventricular tachycardia from high resolution mapping. Circulation (abstract) 1992;86:I-215

25. Boersma L, Brugada J, Schalij M, Kirchhof C, Allessie M: The effects of $\mathrm{K}^{*}$ on anisotropic conduction in sheets of perfused rabbit ventricular epicardium. J Cardiovasc Electrophysiol $1991 ; 2: 492-502$

26. Schalij MJ: Anisotropic conduction and ventricular tachycardia. Doctoral Thesis. University of Limburg, Maastricht, The Netherlands, 1988.

27. Bernstein R, Frame L: Ventricular reentry around a fixed barrier. Resetting with advancement in an in vitro model. Circulation 1990;81:267-280

28. Allessie M, Schalij M, Kirchhof C, Boersma L, Huyberts M, Hollen J: Electrophysiology of spiral waves in two dimensions: the role of anisotropy. Ann NY Acad Sci 1990;591:247-256

29. Wit A, Dillon S: Anisotropic reentry. In: Cardiac Electrophysiology, from cell to bedside. eds Jalife J, Zipes D. W.B. Saunders Company pp. 537-553, 1990

30. Pertsov A, Davidenko J, Salomonsz R, Baxter W, Jalife J: Spiral waves of excitation underlie reentrant activity in isolated cardiac muscle. Circ Res 1993;72:631-650

31. Spach M, Miller W, Geselowitz D, Barr R, Kootsey M, Johnson E: The discontinuous nature of propagation in normal canine cardiac muscle. Evidence for recurrent discontinuities of intracellular resistance that affect the membrane currents. Circ Res 1981;48:35-54

32. Spach M, Miller W, Dolber P, Kootsey M, Sommer J, Mosher C: The functional role of structural complexities in the propagating depolarization in the atrium of the dog. Cardiac conduction disturbances due to discontinuities of effective axial resistivity. Circ Res 1982;50:175-191

33. Spach M, Dolber P, Heidlage J, Kootsey J, Johnson E: Propagating depolarization in anisotropic human and canine cardiac muscle: apparent directional differences in membrane capacitance. A simplified model for selective directional effects of modifying the sodium conductance on Vmax, Tfoot, and the propagation safety factor. Circ Res 1987;60:206-219

34. Osaka T, Kodama I, Tsuboi N, Toyama J, Yamada K: Effects of activation sequence and anisotropic cellular geometry on the repolarization phase of action potential of dog ventricular muscles. Circulation 1987;76:226-236

35. Okumura K, Henthorn R, Epstein A, Plumb V, Waldo A. Further observations on transient entrainment: importance of pacing site and components of the reentrant circuit. Circulation 1985;72:1293-1307

36. Boersma $\mathrm{L}_{i z}$ Brugada J, Kirchhof $\mathrm{C}$, Allessie M: Entrainment of reentrant ventricular tachycardia in anisotropic rings of rabbit myocardium. Mechanisms of termination, changes in morphology and acceleration. Circulation 1993;88:1852-1865

37. Michelson E, Spear J, Moore E: Electrophysiologic and anatomic correlates of sustained ventricular tachyarrhythmias in a model of chronic myocardial intarction. Am J Cardiol $1980 ; 45: 583-590$

38. Karagueuzian $\mathrm{H}$, Fenoglio J, Weiss M, Wit A: Protracted ventricular tachycardia induced by premature stimulation of the canine heart after coronary artery occlusion and reperfusion. Circ Res 1979;44:833-846

39. Ursell P, Gardner P, Albala A, Fenoglio J, Wit A: Structural and electrophysiological changes in the epicardial border zone of myocardial infarcts during infarct healing. Circ Res 1985;56:436-452 


\section{CHAPTER 8}

ENTRAINMENT OF REENTRANT VENTRICULAR TACHYCARDIA IN ANISOTROPIC RINGS OF RABBIT MYOCARDIUM.

Mechanisms of Termination, Changes in Morphology and Acceleration.

Lucas Boersma

Josep Brugada Charles Kirchhof

Maurits Allessie

Circulation 1993;88:1852-1865 


\section{INTRODUCTION}

Programmed electrical stimulation is an important tool to obtain information about the mechanisms of ventricular tachycardia (VT). The ability to initiate and terminate VT by single or multiple premature stimuli is regarded as evidence that reentry is the most likely cause for this arrhythmia. ${ }^{1-3}$ In addition, Waldo et al.$^{4,5}$ and Henthorn et al. ${ }^{6}$ have provided four criteria for entrainment to demonstrate indirectly that an arrhythmia is due to reentry. However, Okumura et al. ${ }^{7}$ and Brugada and Wellens ${ }^{8}$ have pointed out some limitations of using entrainment as a diagnostic tool.

Entrainment is also widely used in the treatment of VT since it terminates VT in about $60 \%$ of cases. However, proarrhythmic effects like acceleration or changes in morphology of VT are not rare. ${ }^{9}$ A number of studies has provided direct evidence for the different mechanisms underlying these events. ${ }^{10-14}$ Several investigations have shown that after myocardial infarction the reentrant circuit comprises an area of slow conduction ${ }^{15-20}$ and that termination of VT by entrainment is due to conduction block in the area of slow conduction. ${ }^{10,21,22}$

To further elucidate the mechanisms involved in both termination of VT or proarrhythmic effects of entrainment, we performed high resolution mapping during entrainment of VT in rings of anisotropic ventricular myocardium. ${ }^{23}$ Our results show that depending on the pacing site and the site and incidence of conduction block, different activation patterns may lead to either termination, change in morphology, or acceleration of VT.

\section{METHODS}

\section{Preparation}

In 18 Langendorff-perfused rabbit hearts, a thin layer of left ventricular subepicardium was created by cryoprocedure. The perfusion system and the cryoprocedure have been described in detail in chapter 2 . In the thin epicardial layer, a fixed anatomical obstacle $(25 \times 10 \mathrm{~mm})$ was created, as described in chapter 4 .

\section{Recording and Stimulation}

A spoon-shaped mapping electrode, molded to the epicardial surface of the left ventricle, containing 248 individual silver electrodes (diameter $0.3 \mathrm{~mm}$, resolution 2.25 $\mathrm{mm}$ ), was used to map the ventricular activation. The on-line mapping system to record and analyze the data was described in chapter 3.

Bipolar stimulation could be performed through any selected pair of electrodes in the electrode spoon. Sustained monomorphic ventricular tachycardia was induced by incremental pacing. During $\mathrm{VT}$ after every $10^{\text {th }}$ beat single premature stimuli were applied at four times diastolic threshold to determine the effective refractory period (ERP) at 8 different sites around the ring. The stimulation protocol to determine the ERP has been described in chapter 7 .

Entrainment of VT was studied by applying trains of 10 stimuli (four times diastolic threshold) with a fixed interstimulus interval after every $10^{\text {th }} V T$ beat. The first stimulus of the train did not always capture the ventricle since stimulation was started at random. However, in all cases at least 9 of the 10 stimuli captured the ventricle. In each heart the effects of entrainment on VT were tested at eight pacing sites evenly 
distributed around the ring. The cycle length of the train of stimuli was shortened in steps of $10 \mathrm{~ms}$ until capture was lost or VT was terminated, changed in morphology, or accelerated.

\section{Statistical Analysis}

Results were compared using paired Student's t-test, ANOVA and Bonferroni's t-test when appropriate. P-values of $<0.05$ were taken as statistically significant.

\section{RESULTS}

\section{Mapping of Transient Entrainment of VT}

In 10 experiments the mean cycle length of electrically induced VT was $167 \pm 17 \mathrm{~ms}$. The left panel in figure 8.1 shows a clockwise VT circulating around the central obstacle with a revolution time of $189 \mathrm{~ms}$. In the corridor between the LAD and the obstacle crowding of isochrones occurred between 40 and $150 \mathrm{~ms}$. In this segment of the ring the impulse travelled with a velocity of $19 \mathrm{~cm} / \mathrm{s}$, whereas conduction at the free wall of the left ventricle was as fast as $62 \mathrm{~cm} / \mathrm{s}$. In the right panel the mean local conduction velocities of clockwise VT in 8 hearts are given. Slow conduction of less than $30 \mathrm{~cm} / \mathrm{s}$ was consistently and exclusively found in the segment of the ring between the LAD and the central obstacle. Previously it has been demonstrated that slow conduction in this corridor was due to impulse propagation transverse to the epicardial fiber orientation, while fast conduction occurred at the base and free wall where the impulse propagates parallel to the fiber orientation..$^{23}$ During initiation of VT by rapid pacing, unidirectional block predominantly occurred in the area of slow conduction. Thus, pacing at the base resulted in a clockwise VT $(n=7)$ while pacing at the free wall or the apex initiated a counterclockwise VT $(n=3)$.

Figure 8.2 shows transient entrainment of the VT given in figure 8.1. The ventricle was paced at 170,140 , and $110 \mathrm{~ms}$ interval at a site proximal to the area of slow conduction. The right lower panel shows entrainment at $110 \mathrm{~ms}$ interval at a pacing site distal to the area of slow conduction. From the electrograms given at the top it can be seen that in all cases VT was transiently entrained and resumed its original rhythm after cessation of pacing. At each pacing interval the stimulus initiated both an antidromic and an orthodromic wave. The antidromic wave collided with the clockwise VT wave while the orthodromic wave restarted VT (continuous reset). During entrainment at different pacing intervals the pattern of activation was similar. However, at shorter intervals the antidromic wave invaded a larger portion of the ring (10\% at $170 \mathrm{~ms}, 18 \%$ at $140 \mathrm{~ms}$, and $25 \%$ at $110 \mathrm{~ms}$ ) resulting in a shift of the site of collision further into the circuit. From these maps the four indirect criteria for entrainment ${ }^{4-6}$ can be derived. Constant fusion during entrainment at a given cycle length (first criterion) corresponds to a constant site of collision of the paced antidromic wave. The second criterion (progressive fusion at shorter pacing intervals) is due to the shift of the site of collision further away from the pacing site. The third criterion related to termination of VT will be discussed in the next section. The fourth criterion for entrainment (change in local electrogram morphology with a shorter stimulus to activation time at shorter pacing intervals) is illustrated in the electrograms at the top of figure 8.2. During pacing at 170 and $140 \mathrm{~ms}$ the stimulated electrogram showed the same morphology as during VT with a fixed and long stimulus to activation time (small arrows). At $110 \mathrm{~ms}$ suddenly 


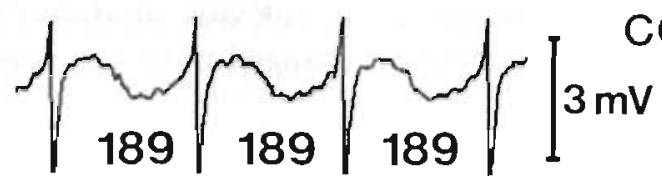

\section{CONDUCTION VELOCITY MAP} $\quad(N=8)$
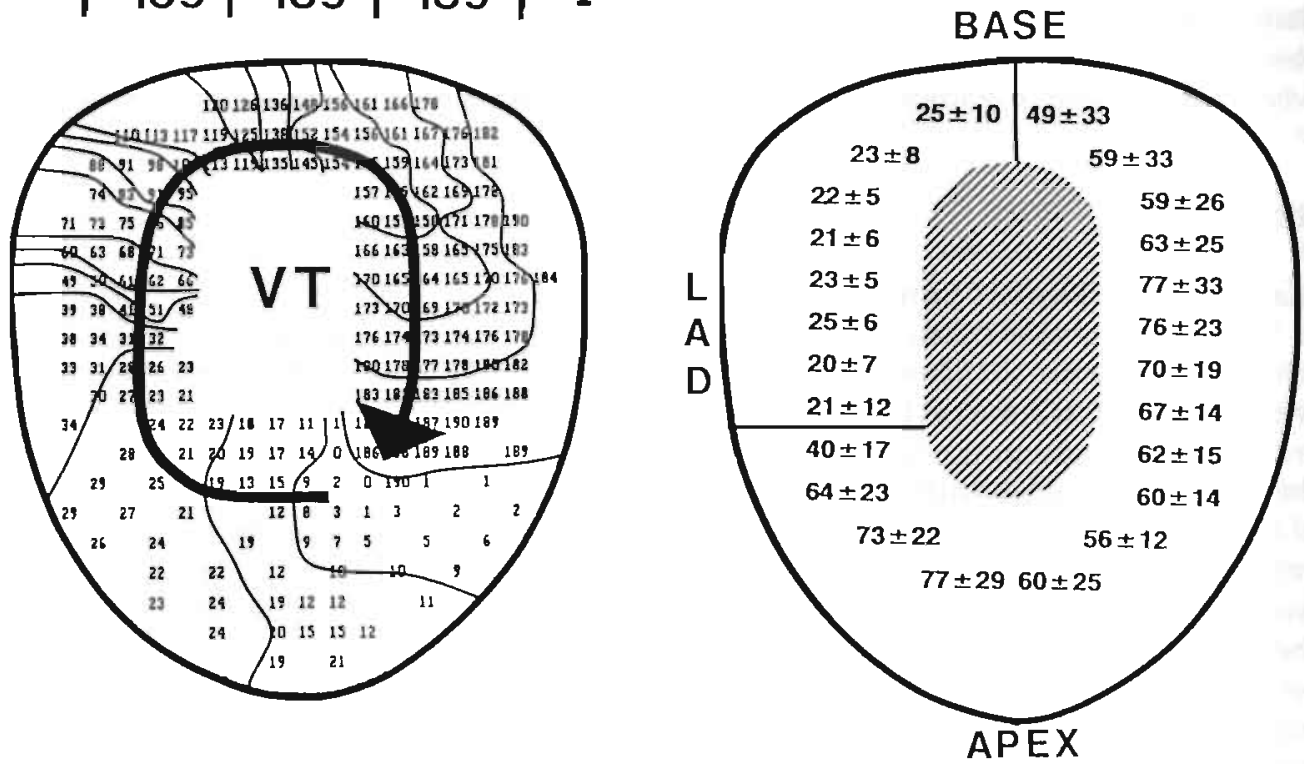

Figure 8.1: Left: Isochrone map during electrically induced clockwise ventricular tachycardia with a revolution time of $189 \mathrm{~ms}$ (electrogram above). Local activation times are given in milliseconds and isochrones are drawn at $10 \mathrm{~ms}$ interoals. The amplitude of the electrogram is given in millivolts. Crowding of isochrones between the $L A D$ and the central obstacle indicates a segment of slow conduction. Right: Map of average local conduction velocities during clockwise VT in 8 different hearts. Two lines at the base and the LAD demarcate the segment of the ring where conduction velocity was less than $30 \mathrm{~cm} / \mathrm{s}$. Conduction velocities are given in $\mathrm{cm} / \mathrm{s}$. LAD: Left Anterior Descending coronary artery.

the stimulus to activation time became much shorter and the electrogram morphology was changed completely. The maps show that these changes were caused by a change from orthodromic to antidromic activation at the recording site (asterisk). The lower maps of figure 8.2 illustrate why these criteria for entrainment are not always fullfilled. Both activation maps show manifest entrainment of VT at $110 \mathrm{~ms}$ interval during pacing proximal and distal to the segment of slow conduction. At the proximal pacing

Figure 8.2 (right): Transient entrainment of a clockwise VT (cycle length $189 \mathrm{~ms}$ ) at pacing intervals of 170 . 140, and $110 \mathrm{~ms}$. The electrograms at the top were recorded at the site indicated in the maps by an asterisk. The small arrows at the end of each train of stimuli indicate the stimulus to activation time at the site of recording. The four maps at the bottom show the activation patterns during transient entrainment. In the lower right map the pacing site was distal to the area of slow conduction whereas in the other maps it was proximal. The moment of stimulation was taken as $t=0$. Activation times are given in milliseconds and isochrones are drawn at $10 \mathrm{~ms}$ intervals. The amplitude of the electrograms is given in millivolts. Black arrows indicate the different wave fronts. See text for description. 


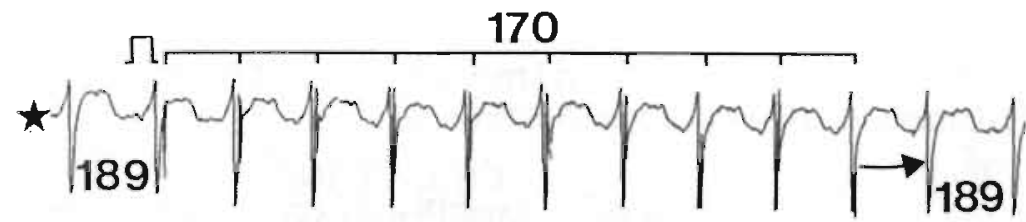

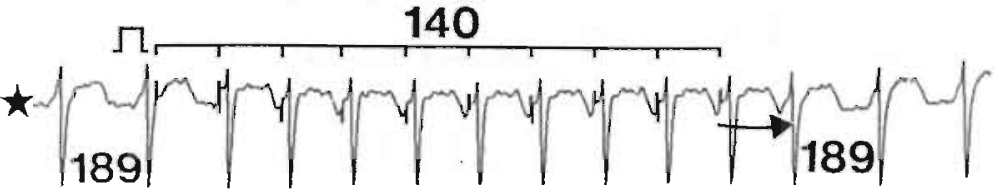
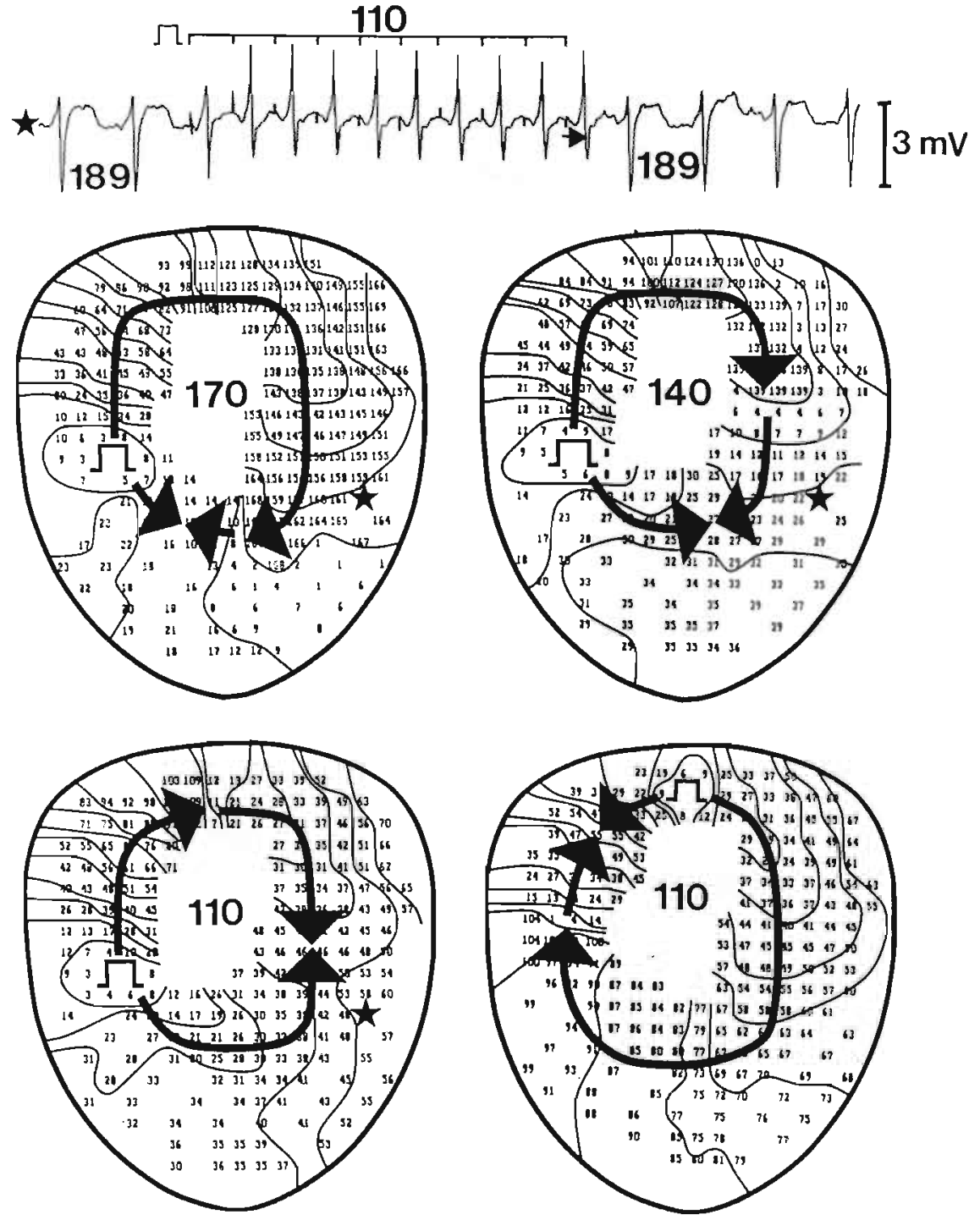


\section{Table 8.1:}

Effects of Entrainment at Short Pacing intervals on VT.

\begin{tabular}{|c|c|c|c|c|c|}
\hline \multirow[t]{2}{*}{ EXP } & \multirow[t]{2}{*}{$\begin{array}{c}\text { NO } \\
\text { CHANGE }\end{array}$} & \multicolumn{2}{|c|}{ TERMINATION } & \multirow[t]{2}{*}{$\begin{array}{l}\text { CHANGE IN } \\
\text { MORPHOLOGY }\end{array}$} & \multirow[t]{2}{*}{ ACCELERATION } \\
\hline & & Block & Echo & & \\
\hline 1. & - & 6 & 2 & - & - \\
\hline 2. & 1 & 5 & - & 1 & 1 \\
\hline 3. & 2 & 4 & - & 2 & - \\
\hline 4. & 3 & 3 & - & - & 2 \\
\hline 5. & - & 5 & 2 & 1 & - \\
\hline 6. & 1 & 3 & 2 & 2 & - \\
\hline 7. & - & 6 & 1 & 1 & - \\
\hline 8. & - & 6 & 1 & 1 & - \\
\hline 9. & 3 & 2 & - & - & 3 \\
\hline \multirow[t]{3}{*}{10.} & 2 & 5 & 1 & - & - \\
\hline & & 45 & 9 & & \\
\hline & 12 & \multicolumn{2}{|c|}{54} & 8 & 6 \\
\hline
\end{tabular}

$E X P=$ experiment number; Block $=V T$ termination by orthodromic conduction block; $E c h o=V T$ termination by an echo-wave

site (left map) the antidromic wave invaded more than $25 \%$ of the circuit while pacing distal to the area of slow conduction resulted in antidromic activation of only $8 \%$ of the ring (right panel). As a result, during pacing distal to the segment of slow conduction almost the entire heart remained activated by the orthodromic wave. It can be expected that in the surface ECG this will lead to only a minimal amount of QRS fusion, resulting in concealed entrainment of VT.

\section{Effects of Entrainment on VT}

Table 8.1 gives a summary of the effects of entrainment on VT $(n=10)$ during rapid pacing at 8 different pacing sites. At 12 of 80 pacing sites in 6 hearts entrainment at the shortest possible pacing interval did not affect VT. At 54 pacing sites rapid pacing terminated VT, while in 8 cases the morphology of VT was changed. In 6 cases VT was accelerated after entrainment.

\section{Termination of VT}

At 54 pacing sites entrainment at short pacing intervals terminated VT (Table 8.1). In 45 of these cases termination was due to complete conduction block of the paced orthodromic wave front. The average interval at which block occurred was $104 \pm 11 \mathrm{~ms}$. 
Entrainment

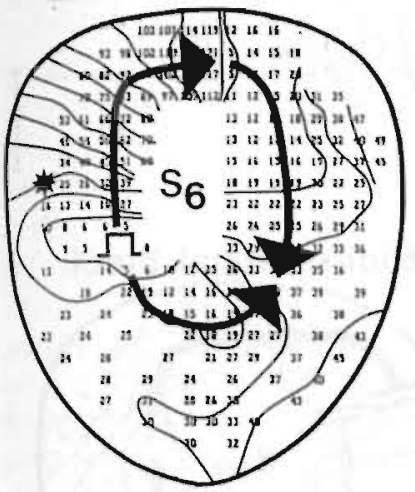

Termination
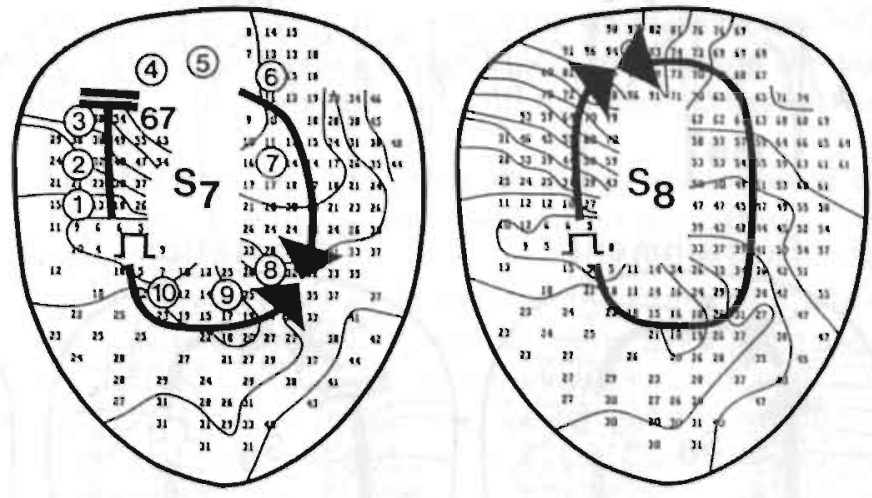
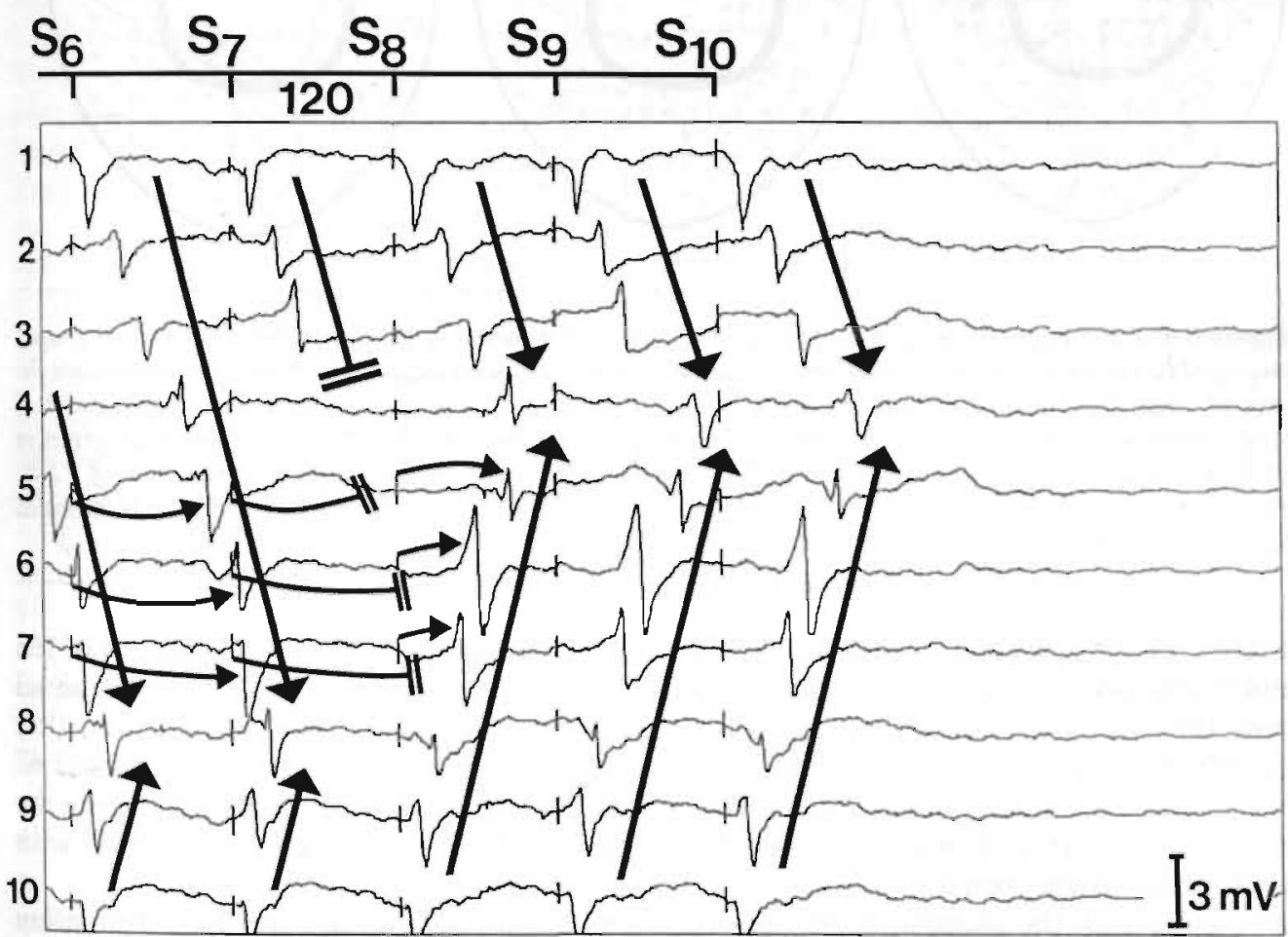

Figure 8.3: Three maps showing entrainment, termination, and collision during rapid pacing (120 ms interoal) of a clockwise VT with a cycle length of $168 \mathrm{~ms}$. The moment of stimulation was taken as $t=0$. Activation times are given in milliseconds and isochrones are drawn at $10 \mathrm{~ms}$ interwals. In the middle map the recording sites of the electrograms shown at the bottom are indicated by' encircled numbers. Small arrows in the electrograms indicate local stimulus to activation time. The amplitule of the electrograms is given in millivolts. Conituction block is indicated by double bar. See text for description. 

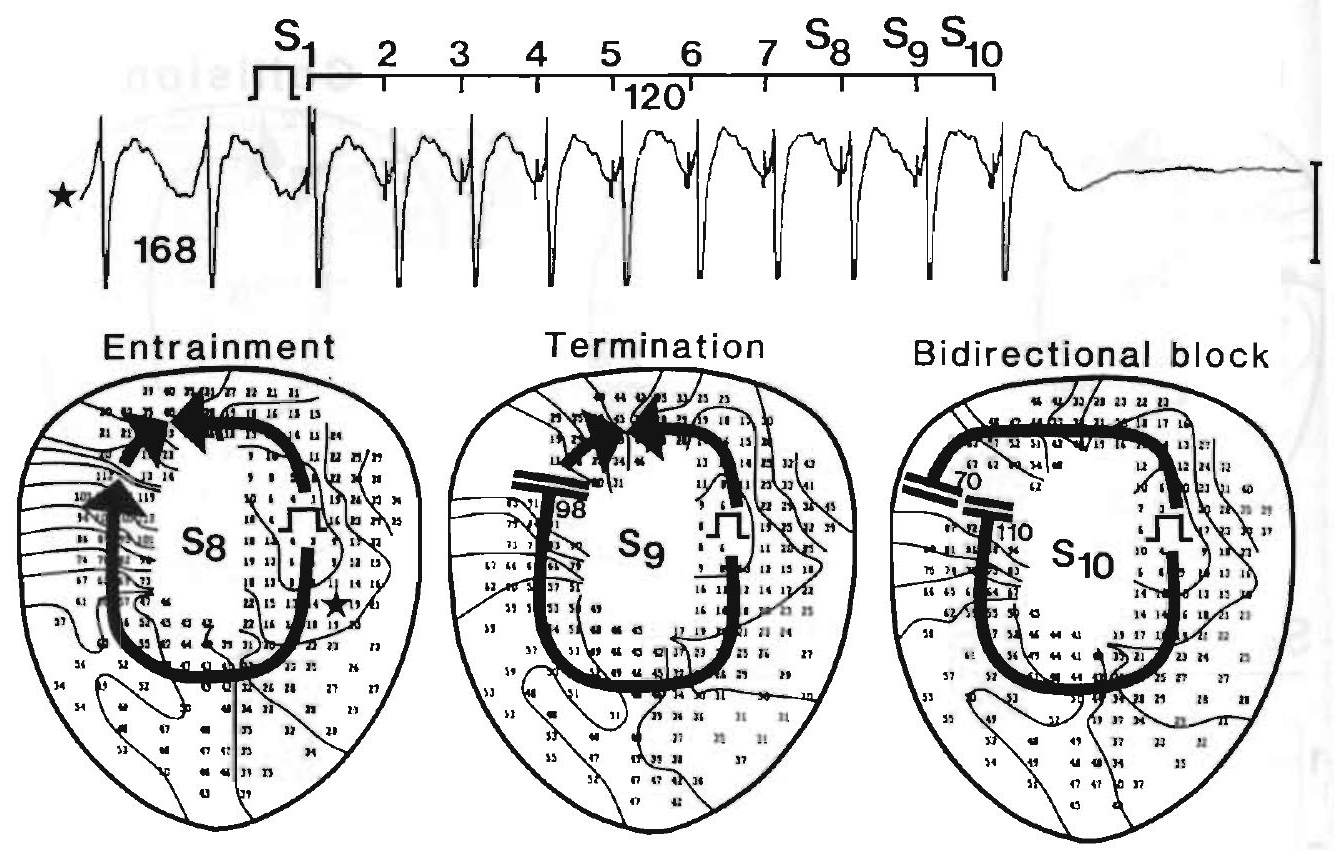

Figure 8.4: Three maps showing bi-directional block after termination of VT (cycle length $168 \mathrm{~ms}$ ) by rapid pacing $(120 \mathrm{~ms})$ of the same VT as given in figure 3 at a different stimulation site. Black arrows indicate the several wave fronts while conduction block is indicated by a double bar. In each map the moment of stimulation was taken as $t=0$ and isochrones are drawn at $10 \mathrm{~ms}$ intervals. Activation times are given in milliseconds and are enlarged at the site of block. The electrogram at the top showing termination of VT by entrainment was recorded at the site indicated by the asterisk. The amplitude of the electrogram is indicated by a $3 \mathrm{mV}$ calibration bar. See text for description.

Figure 8.3 gives an example of termination of a clockwise VT with a cycle length of 168 ms by entrainment at an interval of $120 \mathrm{~ms}$. During the first 6 stimuli VT was entrained and the clockwise circulating wave collided with the paced antidromic wave (left panel). During the $7^{\text {th }}$ stimulus (middle panel) the orthodromic wave was blocked 67 ms after the stimulus was given and the circulating wave was extinguished. During the next beats the ring was activated by two opposed stimulated waves that collided with each other (right panel).

At the bottom the changes in the electrograms around the circuit during termination are shown. Electrograms 5, 6, and 7 fulfill the third criterion for entrainment (termination of VT associated with conduction block to a site for one beat followed by a change in electrogram morphology and a shorter stimulus to activation time during the subsequent beat ${ }^{4}$ ). The maps show that this was due to a local change from orthodromic to antidromic activation after termination of VT. During $\mathrm{S6}$ electrodes 5 to 7 were activated by the paced orthodromic wave with a long stimulus to activation time and the same electrogram morphology as during VT. After orthodromic conduction block during S7, during S8-S10 the same electrodes were activated by an anti- 
dromic wave with a shorter stimulus to activation time and a different electrogram morphology. Conduction block during S7 also occurred at electrogram 4. but during S8 this electrode remained activated by the paced orthodromic wave. Thus, the third criterion for entrainment was only found in a small segment of the ring; (electrodes 5-7). However, it should be realized that when orthodromic block occurred during the last paced beat, VT was terminated without demonstration of the third criterion for entrainment.

In a minority of cases $(n=6)$, interruption of the circulating wave was not followed by collision but instead by bi-directional block of the two opposed paced wave fronts. The left panel of figure 8.4 shows the normal activation pattern during entrainment of VT. During $\mathrm{S9}$ the paced orthodromic wave was blocked $98 \mathrm{~ms}$ after the stimulus (middle panel). Since in this case the site of orthodromic block was far away from the pacing site, the antidromic wave of S10 arrived already $70 \mathrm{~ms}$ after the stimulus and was blocked due to a short local S9-S10 interval $(92 \mathrm{~ms})$. The orthodromic wave of S10 arrived only $40 \mathrm{~ms}$ later at $\mathrm{t}=110 \mathrm{~ms}$ and therefore was also blocked. This pattern of bidirectional block occurred when the orthodromic stimulus to activation time at the site of block was $98 \pm 15 \mathrm{~ms}(n=6)$ compared to $37 \pm 26 \mathrm{~ms}$ in all other cases $(n=39)(p<0.05)$. In 9 of 54 cases in 5 hearts, termination of VT was not due to complete orthodromic block of the ciculating wave but resulted from reflection of the circulating wave in the ring (echo-wave). An example is given in figure 8.5 in which a clockwise VT (cycle length $189 \mathrm{~ms}$ ) was terminated by entrainment with an interval of $100 \mathrm{~ms}$. During the last stimulus (S10) the normal pattern of entrainment was still present (left panel). From the middle panel it can be seen that after $143 \mathrm{~ms}$ the last entrained orthodromic wave front was blocked in the corridor between the LAD and the obstacle. However, the arc of conduction block did not extend across the entire width of the ring allowing the orthodromic wave to continue. Due to slow conduction at the site of block local reentry occurred and the orthodromic wave was reflected in the ring as an echo-wave. During the next beat (right panel) the counterclockwise echo-wave collided with the ongoing clockwise orthodromic wave thus terminating VT. When such an echo-wave occurred while pacing was continued, the antidromic echo-wave collided with the next paced orthodromic wave thereby also terminating reentrant $\mathrm{V} \Gamma$. Electrograms A-O in figure 8.5 show the changes in local activation during echo-wave termination. During $\$ 9$ and S10 the normal pattern of entrainment was present. However, while the last entrained orthodromic wave reset the circuit it was reflected at electrogram $\mathrm{K}$. The resulting echowave propagated in antidromic direction from electrodes $\mathrm{K}$ to $\mathrm{F}$ where it collided with the ongoing circulating wave thus terminating VT.

\section{Change in Morphology of VT}

At 8 of 80 pacing sites in 6 hearts (Table 8.1), entrainment at short pacing intervals resulted in a change in morphology of VT. This was due to termination of the original VT followed by reinitiation of a new VT circulating in the opposite direction. Figure 8.6 gives an example of a VT with a cycle length of $193 \mathrm{~ms}$ which was entrained with an interval of $100 \mathrm{~ms}$. The tracing at the top shows the change in electrogram morphology after entrainment. The upper left map shows that before entrainment VT had a clockwise activation pattern whereas after entrainment VT was based on counterclockwise reentry (lower right map). The cycle length of the new VT was exactly the same. During the first seven stimuli the normal pattern of entrainment occurred. However, during $\mathrm{S} 8$ the orthodromic wave was blocked in the corridor between the LAD and the 


\section{Echo-Wave}

Entrainment

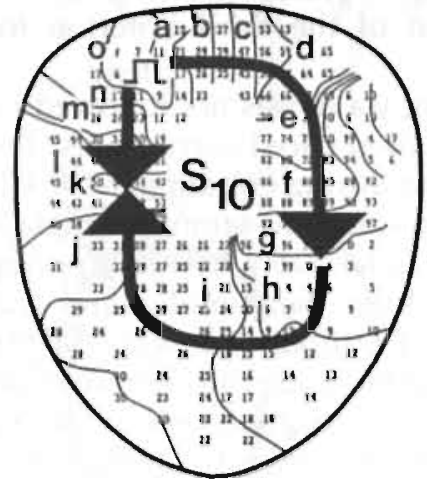

Reflection

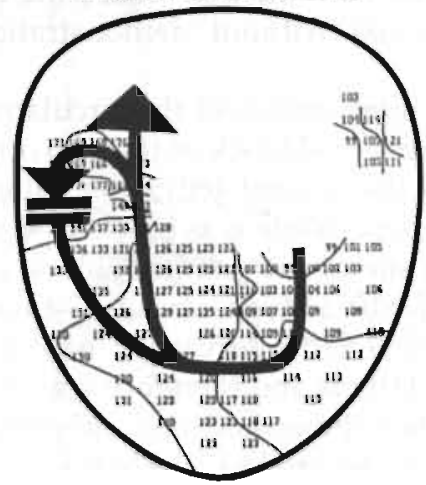

Termination

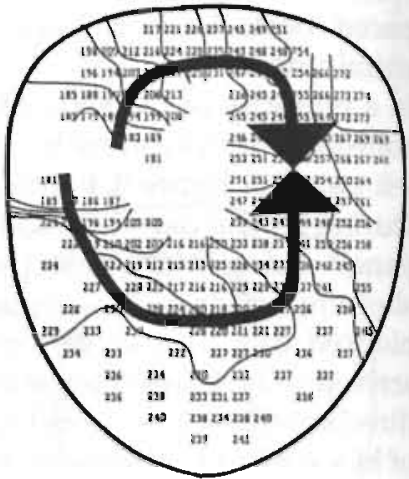

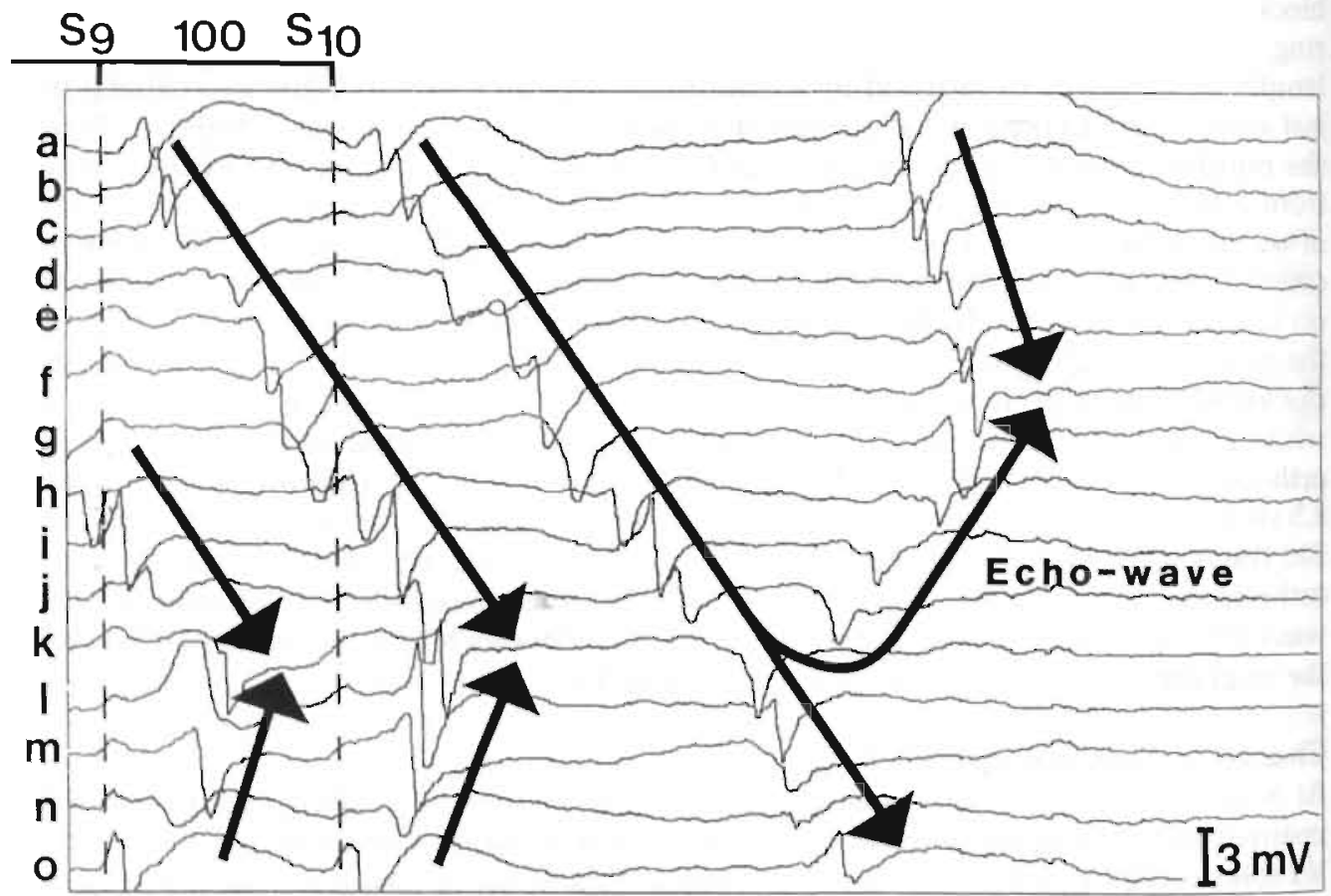

Figure 8.5: Three consecutive maps showing echo-wave termination of VT (cycle length $189 \mathrm{ms,} \mathrm{same} \mathrm{VT}$ as figure 8.1) by entrainment at $100 \mathrm{~ms}$ interval. The moment of stimulation was taken as $t=0$. Activation times are given in milliseconds and isochrones are drawn at $10 \mathrm{~ms}$ intervals. Double bars indicate conduction block. The letters $A$ to $O$ in the left map indicate the recording sites of the 15 electrograms presented at the bottom. The amplitude of the electrograms is indicated by a $3 \mathrm{mV}$ calibration bar. See text for description. 

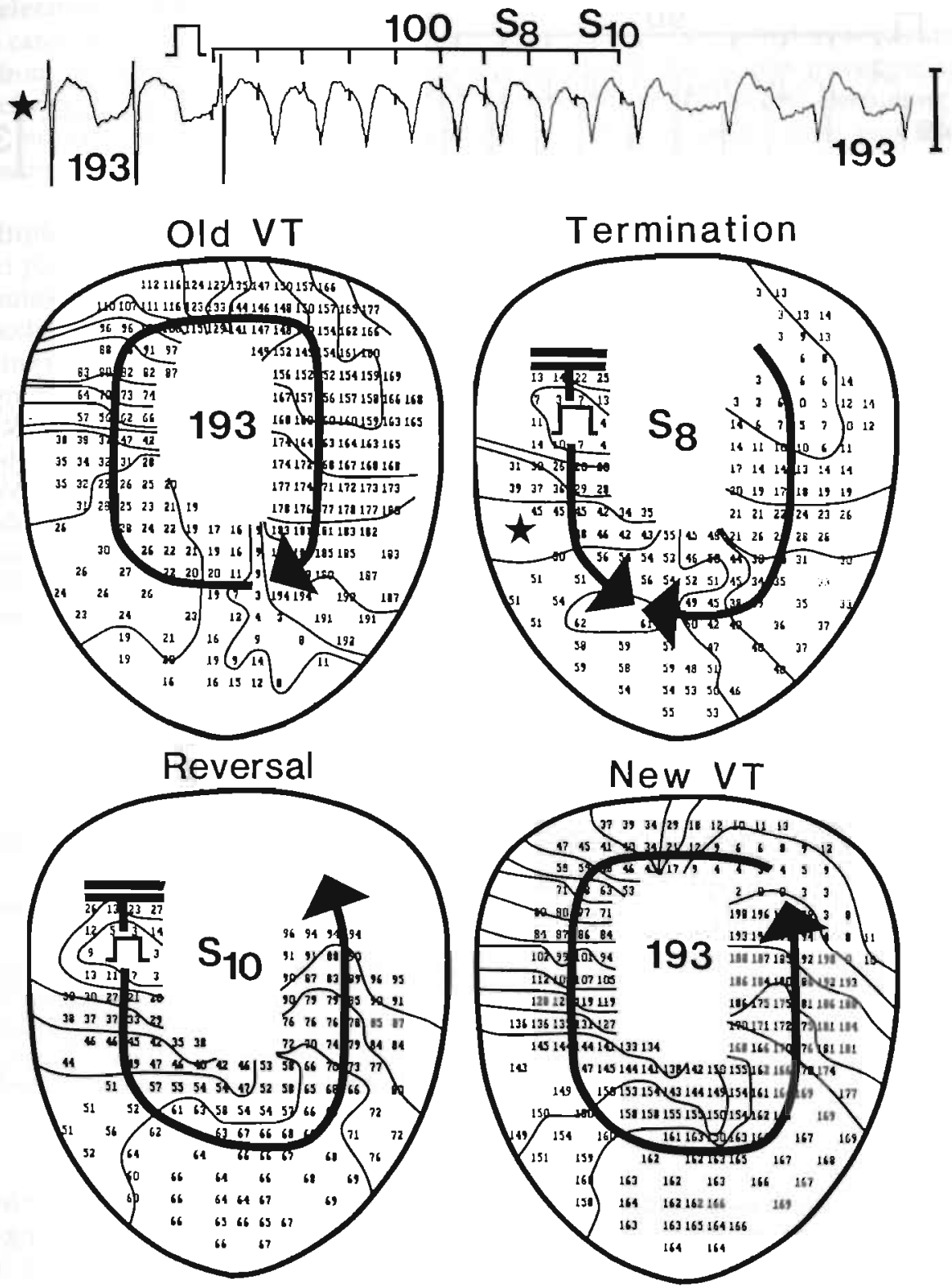

Figure 8.6: A change in morphology of VT (top) after entrainment with an interval of $100 \mathrm{~ms}$. The site of recording is indicated by the asterisk. The original VT (cycle length $193 \mathrm{~ms}$ ) was based on a clockwise circulating wave (upper left map) while after entrainment the direction of propagation had reversed (loweir right map). The other two maps show termination and reinitiation of VT by conduction block both at the same site during $S 8$ and S10. The moment of stimulation was taken as $t=0$ and isochrones are drawn at 10 $\mathrm{ms}$ interoals. The amplitude of the electrogram is indicated by a $3 \mathrm{mV}$ bar. Conduction block is indicated by double bars. See text for description. 


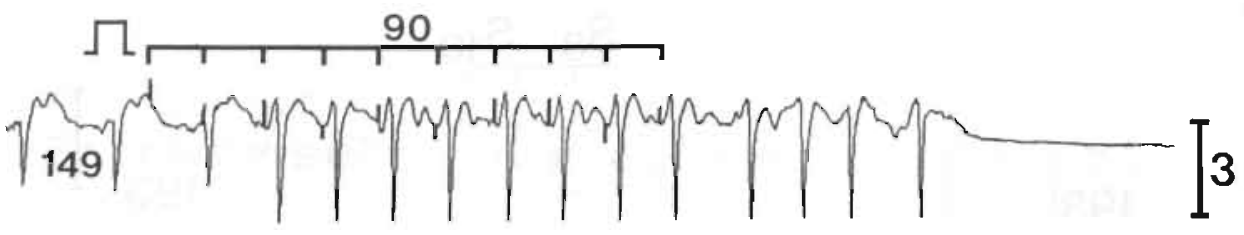

\section{CCW VTEntrainment Termination Collision}
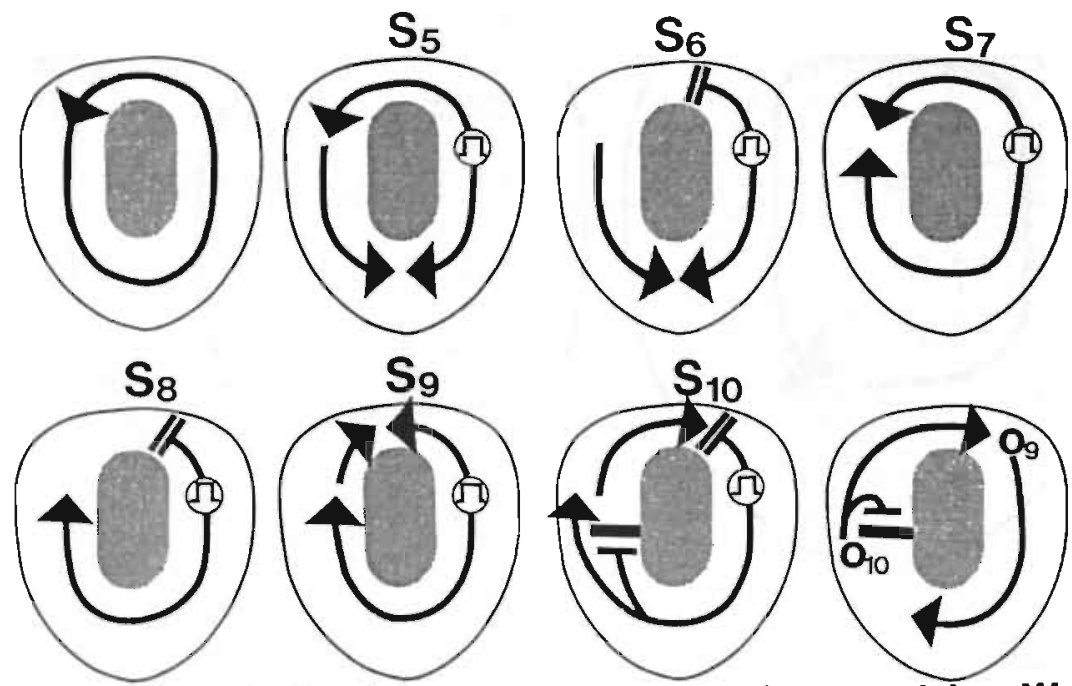

Reversion Entrainment Antidromic block

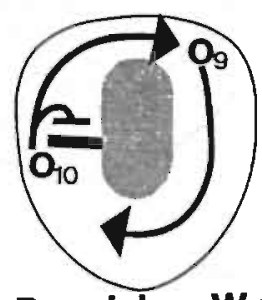

\section{Double-wave Reentry}

Figure 8.7: Complex activation patterns during termination of a counterclockwise VT (cycle length $149 \mathrm{~ms}$ ) by entrainment at $90 \mathrm{~ms}$ interval. Before termination a sequence of Orthodromic block, Reversal, and DoubleWave Reentry occurred. The eight panels give a schematic representation of the activation patterns during S5-S10. The electrogram at the top shows that shortly after cessation of pacing the accelerated VT terminated spontaneously. Black arrows indicate the several waves. Double bars indicate conduction block. The amplitude of the electrogram is given in millivolts. See text for description.

obstacle (upper right map) and VT was terminated. The two opposed paced wave fronts initiated by the next stimulus (S9) collided in the ring (not shown). During 510 conduction block occurred again at the same site in the circuit (lower left map). As a consequence the counterclockwise paced wave front now proceeded unopposed around the ring and reentered the site of block to initiate a countercluckwise VT. In 6 cases of reversal of VT by twice orthodromic block, the third criterion for entrainment was fulfilled because during the beat subsequent to termination of VT, a part of the circuit changed from orthodromic to antidromic activation with a shorter conduction time. In 2 of the 8 cases of a change in morphology of VT, termination of the original VT was followed by reversal of VT by an echo-wave. 


\section{Acceleration of VT}

In 6 cases in 3 hearts (Table 8.1), entrainment at $92 \pm 11 \mathrm{~ms}$ resulted in acceleration of VT from $179 \pm 14$ to $102 \pm 10 \mathrm{~ms}$. Acceleration was due to two waves travelling around the circuit in the same direction (double-wave reentry ${ }^{11}$ ). The second circulating wave was induced when during entrainment the paced antidromic wave was blocked, allowing the previous paced orthodromic wave to continue unopposed.

\section{Multiple Events during Entrainment of VT}

At 21 pacing sites conduction block during entrainment occurred during more than one stimulus, either at the same or at a different site. In 9 of these 21 cases, twice orthodromic block at the same site resulted in reversal of VT in all but 3 cases where bi-directional block prevented reinitiation. In 2 cases, orthodromic block was followed by an echo-wave resulting in a sequence of termination and reversal of VT. In 5 cases, block occurred three times at two different sites resulting in a sequence of termination, reinitiation and termination of $\mathrm{VT}$.

In the remaining 5 cases of entrainment, different combinations of orthodromic block, antidromic block, and echo-waves resulted in highly complex activation patterns during entrainment. Figure 8.7 shows an example in which during one train of stimuli a counterclockwise VT with a cycle length of $149 \mathrm{~ms}$ was subsequently terminated, reversed, and accelerated. During S1-S5 the normal pattern of entrainment occurred. During S6 VT was terminated by orthodromic block followed by collision of the two opposed paced wave fronts during S7. During S8 block of the counterclockwise wave inititiated a clockwise VT, which was entrained during S9. Finally double-wave reentry was induced during S10 due to block of the antidromic wave, followed by reentry by the orthodromic wave of S9. As can be seen in the electrogram at the top, in this case double-wave reentry was not sustained and VT terminated shortly after cessation of pacing. This sequence of events illustrates that block can occur at multiple sites during successive stimuli resulting in a continuously changing activation pattem. Since ventricular activation after cessation of pacing completely depends on the activation pattern during the last stimulus, in this situation the type of VT resulting from entrainment is largely determined by chance.

\section{Role of Local Oscillations in Interval in Termination of VT}

In 35 cases of termination of VT the mechanisms underlying complete orthodromic conduction block were studied. Block occurred predominantly in the corridor between the LAD and the obstacle $(n=28)$. In only 7 cases the site of block was located at the base of the ventricle (figure 8). In 30 of 35 cases conduction velocity during VT at the site of block was less than $30 \mathrm{~cm} / \mathrm{s}(25 \pm 5 \mathrm{~cm} / \mathrm{s})$, while only in 5 cases conduction was rapid $(61 \pm 12 \mathrm{~cm} / \mathrm{s})$. Therefore, during entrainment slow transverse conduction was more vulnerable to conduction block than fast longitudinal propagation. Spatial dispersion in refractoriness could not explain termination of VT. In the majority of cases $(n=24)$ the ERP distal to the site of block was equal to or shorter than proximal to the site of block $(-7 \pm 7 \mathrm{~ms})$. Only in 11 of 35 cases block occurred when the impulse travelled towards an area with a longer refractory period ( $+15 \pm 9 \mathrm{~ms})$.

In all cases of orthodromic block by entrainment, local oscillations at the site preceded termination of VT. On the average, oscillations of $20 \pm 9 \mathrm{~ms}$ were found. The last interval prior to conduction block was always shorter than the interstimulus interval (94 \pm 10 versus $104 \pm 11 \mathrm{~ms}, \mathrm{p}<0.05$ ). An example is given in figure 8.9 . The three 


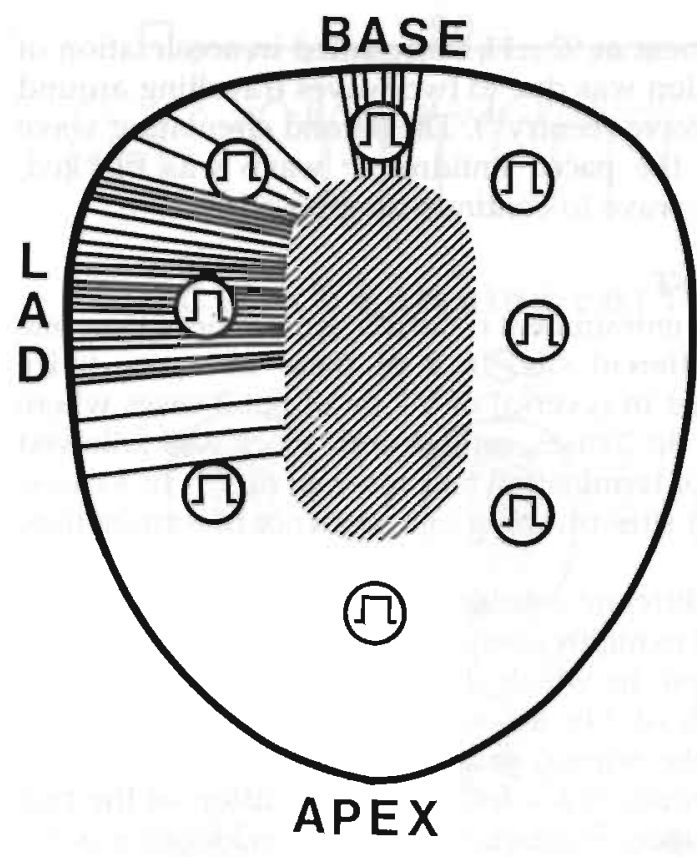

Figure 8.8: Location of the lines of orthodromic conduction block in 35 cases of termination of VT. Termination of VT by entrainment was possible at each pacing site indicated around ring ( П). See text for description.

electrograms at the top were recorded respectively close to the pacing site $(\Pi)$, and proximal (asterisk) and distal (dot) to the site of block. VT with a cycle length of 168 ms was entrained by a train of stimuli with an interval of $120 \mathrm{~ms}$. Since stimulation was started randomly the first stimulus failed to capture the ventricle while the second stimulus resulted in an interval of $140 \mathrm{~ms}$. During all subsequent stimuli, a constant interval of $120 \mathrm{~ms}$ was found at the pacing site (upper tracing). In the electrograms recorded both proximal and distal to the site of block however, clear and progressive oscillations in interval occurred. Due to these oscillations the last interval proximal to the site of block (double bar) was shortened to $113 \mathrm{~ms}$.

The maps at the bottom of figure 8.9 show the segment of the circuit (between the LAD and the central obstacle) where orthodromic block occurred. The conduction times towards the proximal and distal electrodes increased from 64 and 78 during S3 to 71 and $89 \mathrm{~ms}$ during S4, resulting in a prolongation of the local intervals from 120 to 127 and $131 \mathrm{~ms}$ respectively. During S5 the conduction time was shorter again resulting in a compensatory shortening in interval. This short interval induced a small line of block at the edge of the ring (thick line) which however did not terminate VT. During the next stimuli the same phenomena as during S4 and S5 occurred, resulting in further shortening in local interval to $113 \mathrm{~ms}$. This short interval resulted in complete conduction block across the entire width of the ring (double bar) and VT termination.

\section{DISCUSSION}

In previous studies Waldo et al. ${ }^{15}$ and Henthorn et al. ${ }^{6}$ defined four criteria for entrainment of tachycardia to provide indirect evidence that the arrhythmia is based on 

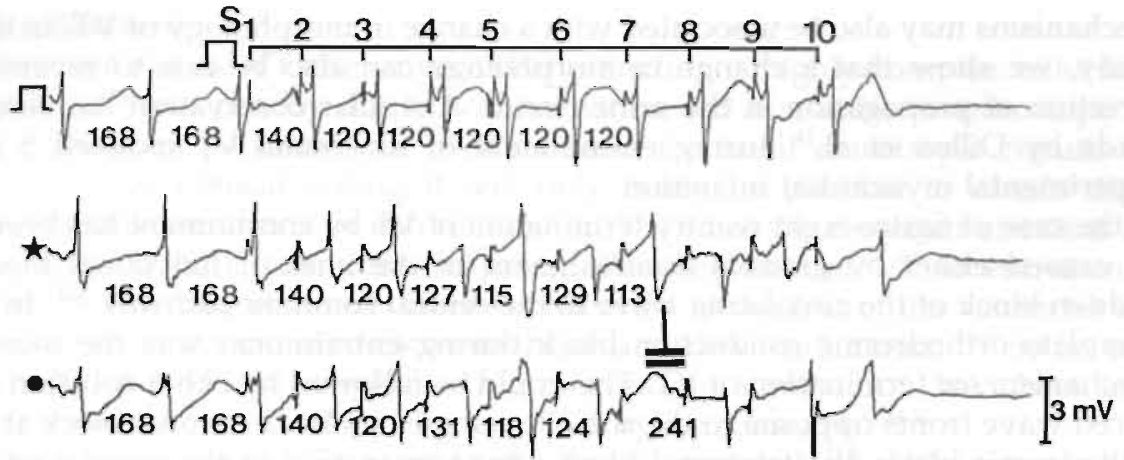

ACTIVATION MAPS

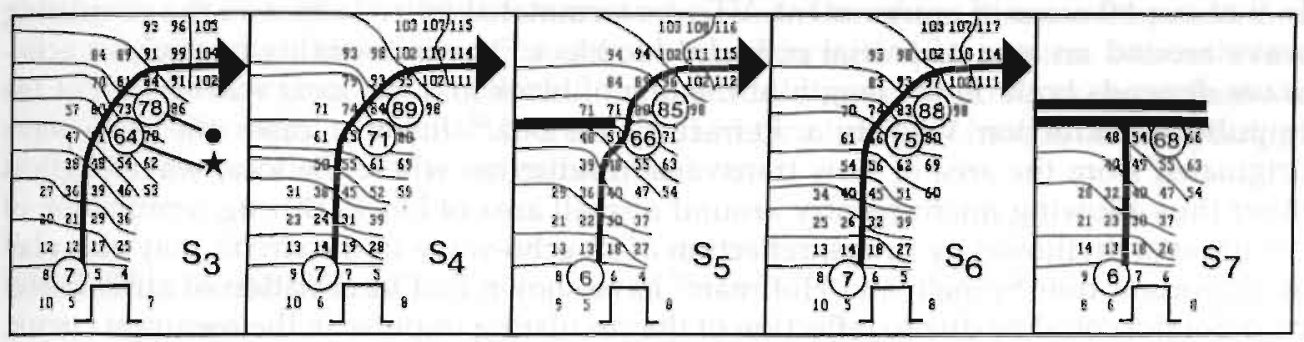

Figure 8.9: Local oscillations in interval during entrainment of VT leading to termination of VT. The VT had cycle length of $168 \mathrm{~ms}$ and was entrained with an interval of $120 \mathrm{~ms}$. The three electrograms at the top show the local responses recorded next to the pacing site ( $\Pi$ ), and at a site proximal (asterisk) and distal (dot) to the line of conduction block. The beat-to-beat intervals are given at each electrogram. The maps at the bottom show the segment of the circuit between the LAD and the central obstacle during S3-S7. The local activation times at the three recording sites are encircled and enlarged. The moment of stimulation was taken as $t=0$, activation times are given in ms and isochrones are drawn at $10 \mathrm{~ms}$ intervals. The amplitude of the electrograms is given in millivolts. Double bars indicate conduction block. See text for description.

reentry. These criteria are based on the concept that during transient entrainment of reentry the circuit is invaded both in orthodromic and antidromic direction. The first results in acceleration of VT to the pacing rate while the latter causes a change in QRSmorphology in the surface ECG. The activation patterns during entrainment in the present study provide direct support for the validity of the four criteria for entrainment. Okumura et al. ${ }^{721}$ have shown that none of the four criteria were fulfilled during pacing distal to an area of slow conduction within a reentrant circuit. Our results may explain their finding by showing that if pacing was performed distal to an area of slow conduction antidromic invasion of the circuit only occurred over a length of $8 \%$. Thus, despite acceleration of VT to the pacing rate, the direction of activation of the majority of the ventricle remained the same resulting in concealed entrainment.

Only a limited number of studies has provided direct evidence for the mechanisms responsible for acceleration, change in morphology or termination of VT by entrainment. Acceleration of VT can be due to either double-wave reentry ${ }^{11,12}$, a change to another anatomical circuit ${ }^{12,24}$ or induction of functional reentry. ${ }^{10,11,14}$ The last two 
mechanisms may also be associated with a change in morphology of VT. In the present study, we show that a change in morphology can also be due to reversion of the direction of propagation in the same circuit. A similar observation has recently been made by Dillon et al. ${ }^{14}$ during entrainment of functional VT induced 5 days after experimental myocardial infarction.

In the case of figure-eight reentry termination of VT by entrainment has been found to be caused either by gradual diminishment of the lines of functional block ${ }^{13}$ or by sudden block of the circulating wave in the central common pathway. ${ }^{10,13}$ In our study complete orthodromic conduction block during entrainment was the most frequent mechanism for termination of VT. This could be followed by either collision of the two paced wave fronts opposite to the site of pacing or by bi-directional block at the site of orthodromic block. Bi-directional block after termination of the circulating wave has also been observed during entrainment of atrial flutter. ${ }^{25}$

In 9 of our 80 cases of entrainment, VT was terminated by reflection of the circulating wave around an area of partial orthodromic block. The inducibility of such an echowave depends both on the length of the line of block and the local wavelength of the impulse (Conduction Velocity $x$ Refractory Period). ${ }^{26}$ In most cases the echo-wave originated from the area of slow transverse conduction where the local wavelength is short thus allowing micro-reentry around a small area of block. During termination of reentrant arrhythmias by drugs, reflection of an echo-wave in the circuit may also play an important role. Spinelli and Hoffman ${ }^{27}$ have shown that termination of atrial flutter by $d$-Sotalol could be due to reflection of the circulating impulse in the reentrant circuit. In the same model of VT as used in our study, Brugada et al. ${ }^{28}$ showed that in $43 \%$ of cases termination of VT by various anti-arrhythmic drugs was caused by an echo-wave.

\section{The Role of an area of Slow Conduction in Termination of VT}

Many investigators ${ }^{15-20}$ have provided evidence that during VT an area of slow conduction is present within the reentrant circuit. Abnormal tissue properties in this area of slow propagation have been held responsible for termination of $\mathrm{VT}^{10,21,22}$ In contrast, Waldecker et al. ${ }^{13}$ recently reported that termination of functional VT did not occur in the area of slow conduction. In our experiments VT was predominantly terminated by block in a segment of the circuit showing slow transverse conduction. Also during initiation of VT by rapid pacing conduction block occurred almost exclusively in the corridor between the LAD and the central obstacle. Since the refractory period in the area of slow conduction was not significantly different from other parts of the ring, it seems unlikely that spatial dispersion in refractoriness was responsible for termination of VT. Instead, preferential transverse rate-dependent conduction block seems to play a role.

Prior to termination of VT by entrainment clear oscillations in cycle length were observed at the site of block. These oscillations culminated in a very short last local interval causing block of the circulating wave. In a model of atrial flutter, Frame et al. ${ }^{29,30}$ have observed that spontaneous termination of atrial flutter was also preceded by oscillations in interval. They showed that these interval oscillations induced concommitant changes in action potential duration and diastolic interval. Termination of atrial flutter was thought to be due to the combination of a locally prolonged refractory period followed by arrival of the next impulse with a shorter interval. In our experiments similar oscillations in refractory period might have contributed to termination of VT. 


\section{Clinical implications}

The substrate for clinical VT after myocardial infarction is highly complex, exhibiting a high degree of heterogeneity due to scar formation and non-uniform anisotropy. ${ }^{17}$ Both macro-reentrant pathways ${ }^{19}$ as well as areas for small anisotropic circuits are available. ${ }^{15-18}$ In the clinical setting it will only rarely be possible to obtain direct evidence of the precise effects of entrainment on VT, since the reentrant circuit is not easily accessible to high resolution mapping. The response of VT to entrainment depends on the way the reentrant circuit is connected to the rest of the ventricles and on the position of the pacing site relative to the area of slow conduction. ${ }^{7,31,32}$ So far only indirect criteria derived from single electrograms and the surface ECG have been used to characterize the events during a change of VT by entrainment. ${ }^{22,24,30}$

Our experimental model of VT consisted of a ring of completely healthy myocardium exhibiting a normal degree of uniform anisotropy. Entrainment was always performed at a pacing site located within the reentrant circuit. Therefore, direct extrapolation of our findings to the clinical situation should be performed with caution. Nevertheless, the detailed mapping data obtained in our study may well serve as a basis to understand the various mechanisms involved in termination, changes in morphology or acceleration of clinical VTs by entrainment.

\section{CONDENSED ABSTRACT}

In 10 Langendorff perfused rabbit hearts, reentrant VT was induced around rings of anisotropic epicardium. At low pacing rates VT was transiently entrained but resumed its normal pattern after cessation of pacing. During entrainment at shorter pacing intervals VT either terminated $(82 \%)$, accelerated $(8 \%)$ or changed in morphology $(10 \%)$. All changes were due to conduction block occurring preferentially during slow transverse propagation. Local oscillations in interval resulted in orthodromic block of the circulating wave and termination of VT. In $16 \%$ of the cases VT terminated by an echo-wave. Acceleration of VT was due to double-wave reentry. Changes in morphology of VT were caused by a reversion of the direction of propagation in the circuit. 


\section{REFERENCES}

1. Wellens HJJ, Schuilenburg R, Durrer D: Electrical stimulation of the heart in patients with ventricular tachycardia. Circulation 1972;46:216-226

2. Wellens HJJ: Value and limitations of programmed electrical stimulation of the heart in the study and treatment of tachycardias. Circulation 1978;57:845-853

3. Josephson M, Horowitz L, Farshidi, Kastor J: Recurrent sustained ventricular tachycardia: 1. Mechanisms. Circulation 1978;:57:431-439

4. Waldo A, Plumb V, Arciniegas J, MacLean W, Cooper T, Priest M, James T: Transient entrainment and interruption of the atrioventricular bypass pathway type of paroxysmal atrial tachycardia. Circulation 1983;67:73-83

5. Waldo $A$ and Henthorn R: Use of transient entrainment during ventricular tachycardia to localize a critical area in the reentry circuit for ablation. PACE 1989;12:231-244

6. Henthorn R, Okumura K, Olshansky B, Plumb V, Waldo A: A fourth criterion for transient entrainment: the electrogram equivalent of progressive fusion. Circulation 1988;77:1003-1012

7. Okumura K, Henthorn R, Epstein A, Plumb V, Waldo A: Further observations on transient entrainment: importance of pacing site and properties of the components of the reentrant circuit. Circulation 1985;72:1293-1307

8. Brugada P and Wellens HJJ: Entrainment as an electrophysiologic phenomenon. J Am Coll Card $1984 ; 3: 451-454$

9. Waldecker B, Brugada P, Zehender M, Stevenson W, Den Dulk K, Wellens HJJ: Modes of electrical termination of ventricular tachycardia: importance for the selection of implantable antitachycardia device. Am J Cardiol 1986-57:150-155

10. El-Sherif N, Gough W, Restivo M: Reentrant ventricular arrhythmias in the late myocardial infarction period:14. Mechanisms of resetting, entrainment, or acceleration of reentrant tachycardia by programmed electrical stimulation. PACE 1987;10:341-371

11. Brugada J, Boersma L, Kirchhof $C$, Brugada P, Havenith $M$, Wellens $H$, Allessie M: Double-wave reentry as a mechanism of acceleration of ventricular tachycardia. Circulation 1990;81:1633-1643

12. Brugada J, Brugada P, Boersma L, Mont Ll, Kirchhof C, Wellens HJ, Allessie MA: On the mechanisms of ventricular tachycardia acceleration during programmed electrical stimulation. Circulation 1991;83:1621-1629

13. Waldecker B, Coromilas J, Saltman A, Dillon S, Wit A: Overdrive stimulation of functional reentrant circuits causing ventricular tachycardia in the infarcted canine heart. Resetting and entrainment. Circulation 1993;87:1286-1305

14. Dillon S, Coromilas J, Waldecker B, Wit A: Effects of overdrive stimulation of reentrant circuits causing ventricular tachycardia in the infarcted heart. Mechanisms for resumption or alteration of tachycardia. J Cardiovasc Electrophys 1993 (in press)

15. El-Sherif N, Scherlag B, Lazzara R, Hope R: Reentrant ventricular arrhythmias in the late myocardial infarction period. 1. Conduction characteristics in the infarction zone. Circulation 1977;55:783-791

16. Wit A, Allessie M, Bonke F, Lammers W, Smeets J, Fenoglio J: Electrophysiologic mapping to determine the mechanism of experimental ventricular tachycardia initiated by premature impulses. Am J Cardiol 1982;49:166-185

17. Ursell P, Gardner P, Albala A, Fenoglio J, Wit A:Structural and electrophysiological changes in the epicardial border zone of myocardial infarcts during infarct healing. Circ Res 1985;56:436-452

18. Dillon $S$, Allessie M, Ursell $P$, Wit A: Influences of anisotropic tissue structure on reentrant circuits in the epicardial borderzone. Circ Res 1988;63:182-206

19. de Bakker J, van Capelle F, Janse M, Wilde A, Coronel R, Becker A, Dingemans K, van Hemel N, Hauer R: Reentry as a cause of ventricular tachycardia in patients with chronic ischemic heart disease. Electrophysiologic and antomic correlation. Circulation 1988;77:589-606

20. Littmann L, Svenson R, Gallagher J, Selle J, Zimmern S, Fedor J, Colavita: Functional role of the epicardium in postinfarction ventricular tachycardia. Observations derived from computerized epicardial activation mapping, entrainment, and epicardial laser photoablation. Circulation $1991 ; 83: 1577-1591$

21. Okumura K, Olshansky B, Henthorn R, Epstein A, Plumb V, Waldo A: Demonstration of the presence of slow conduction during sustained ventricular tachycardia in man: use of transient 
entrainment. Circulation 1987;75:369-378

22. Aizawa $Y$, Niwano S, Chinushi M, Tamura M, Kusano Y, Miyajima T, Kitazawa H, Shibata A: Incidence and mechanism of interruption of reentrant ventricular tachycardia with rapid ventricular pacing. Circulation 1992;85:589-595

23. Brugada J, Boersma L, Kirchhof C, Heynen V, Allessie MA: Reentrant excitation around a fixed obstacle in uniform anisotropic ventricular myocardium. Circulation 1991;84:1296-1306

24. Fitzgerald D, Friday K, Yeung-Lai-Wah J, Bowman A, Lazzarra R, Jackman W: Myocardial regions of slow conduction participating in the reentrant circuit of multiple ventricular tachycardias: Report on ten patients. J Cardiovasc Electrophysiol 1991;2:193-206

25. Boyden $P$, Frame L, Hoffman B: Activation mapping of reentry around an anatomic barrier in the canine atrium. Circulation 1989;79:406-416

26. Rensma P, Allessie M, Lammers W, Bonke F, Schalij M: Length of excitation wave and susceptibility to reentrant atrial arrhythmias in normal conscious dogs. Circ Res 1988;62:395-410

27. Spinelli $W$ and Hoffman B: Mechanisms of termination of reentrant atrial arrhythmias by class $I$ and class III antiarrhythmic drugs. Circ Res 1989;65:1565-1579

28. Brugada J, Boersma L, Kirchhof C, Allessie MA: Echo-Wave termination of ventricular tachycardia: a common mechanism of termination of reentrant arrhythmias by various pharmacological interventions. Circulation 1992;85:1879-1887

29. Frame L and Simson M: Oscillations of conduction, action potential duration, and refractoriness. A mechanism for spontaneous termination of reentrant tachycardias. Circulation 1988;78:12771287

30. Frame $\mathrm{L}$ and Rhee $\mathrm{E}$ : Spontaneous termination of reentry after one cycle or short non-sustained runs. Role of oscillations and excess dispersion of refractoriness. Circ Res 1991;68:493-502

31. Almendral J, Gottlieb C, Rosenthal M, Stamato N, Buxton A, Marchlinski F, Miller J, Josephson M: Entrainment of ventricular tachycardia: explanation for surface electrocardiographic phenoma by analysis of electrograms recorded within the reentrant circuit. Circulation 1988;77:569-580

32. Rosenthal M, Stamato N, Almendral J, Gottlieb C, Josephson M: Resetting of ventricular tachycardia with electrocardiographic fusion: incidence and significance. Circulation 1988;77:581588 


\section{CHAPTER 9}

\section{EFFECTS OF HEPTANOL, CLASS Ic, AND CLASS III DRUGS ON REENTRANT VENTRICULAR TACHYCARDIA.}

Importance of the excitable gap for the inducibility of double-wave reentry.

Lucas Boersma Josep Brugada Charles Kirchhof Maurits Allessie

Submitted 


\section{INTRODUCTION}

In addition to the beneficial effects of antiarrhythmic drugs and overdrive stimulation, also aggravation of preexisting arrhythmias and/or provocation of new arrhythmias have been reported. ${ }^{1,2}$ In $36 \%$ of patients with VT after myocardial infarction overdrive stimulation had a proarrhythmic effect, ${ }^{2}$ while electrophysiological studies in patients on anti-arrhythmic drugs showed an $18 \%$ incidence of proarrhythmias. ${ }^{1}$

A number of experimental studies have elucidated some of the mechanisms of proarrhythmia during overdrive stimulation and antiarrhythmic drug therapy. ${ }^{3-8}$ In previous investigations from our laboratory ${ }^{4.5}$ it has been shown that acceleration of VT by PES can be caused either by a change in reentrant circuit or by induction of a second wave in the same circuitous pathway (double-wave reentry). Double-wave reentry could only be induced in relatively slow VTs with a large excitable gap. We hypothesized that antiarrhythmic drugs might promote or inhibit the inducibility of doublewave reentry by affecting the length of the excitable gap during VT. In the present study we investigated the effects of electrical uncoupling, and class Ic and class III effects both on the excitable gap and the susceptibility to PES-induced acceleration of VT. Our results show that both class Ic drugs and uncoupling agents increase the likelyhood of induction of double-wave reentry by enlarging the excitable gap during VT. On the other hand, class III drugs may prevent acceleration by shortening the excitable gap during VT.

\section{METHODS}

\section{Preparation}

In 11 Langendorff-perfused rabbit hearts, a thin layer of left ventricular subepicardium was created by cryoprocedure. The perfusion system and the cryoprocedure have been described in detail in chapter 2 . In the thin epicardial layer, a fixed anatomical obstacle $(25 \times 10 \mathrm{~mm})$ was created, as described in chapter 4 .

\section{Recording and Stimulation}

A spoon-shaped mapping electrode, molded to the epicardial surface of the left ventricle, containing 248 individual silver electrodes (diameter $0.3 \mathrm{~mm}$, resolution 2.25 $\mathrm{mm}$ ), was used to map the ventricular activation. The on-line mapping system to record and analyze data has been described in chapter 3 .

From the activation maps, longitudinal conduction velocity $\left(\theta_{L}\right)$ was determined at the base and the left free wall of the left ventricle while transverse conduction velocity $\left(\theta_{\mathrm{T}}\right)$ was measured at the corridor between the LAD and the central obstacle. ${ }^{10,11}$ Conduction velocity was calculated from the conduction time across these selected segments of the ring, choosing the exact points of measurement in such a way that the pathway the conduction velocity was determined normal to the direction of the isochrones.

Sustained monomorphic ventricular tachycardia was induced by incremental pacing. Reset and entrainment of VT was performed at three different pacing sites around the ring, at the base, the free wall, and the apex of the left ventricle. Both during control and during infusion of Org7797, d-Sotalol and Heptanol the same pacing protocol was performed. In those hearts where heptanol and Org7797 were tested sequentially, control measurements were performed before Org7797 was infused. 
During VT the effective refractory period (ERP) at three different sites around the ring was measured by applying single premature stimuli ( 4 times threshold) during the tachycardia. The stimulation protocol to measure the ERP has been described in chapter 7. Entrainment of VT was performed by applying trains of 10 stimuli at 4 times threshold, as described in chapter 8 . During each VT about 8 different rates of entrainment were tested.

\section{Drug infusion}

Heptanol (Merck), Org7797 (Organon), and d-Sotalol (Bristol-Myers) were dissolved in Tyrode and added to the perfusion fluid by an infusion pump. The effective concentrations were: Heptanol $1.0 \mathrm{mM}$, Org7797 $0.5 \mu \mathrm{M}$, and d-Sotalol $35 \mu \mathrm{M}(10 \mathrm{mg} / \mathrm{l})$. A 30 minute equilibration time was allowed before testing the electrophysiologic effects of each drug. Heptanol and Org7797 were tested separately in two hearts and sequentially in three hearts. In these three hearts heptanol was administered first, since it was washed out more easily than Org7797. d-Sotalol was administered separately in four other hearts.

\section{Statistical Analysis}

Results were compared using the paired Student's t-test, Analysis of Variance, and Bonferroni's t-test. P-values of less than 0.05 were taken as statistically significant.

\section{RESULTS}

\section{Characteristics of VT during control}

In 11 experiments ventricular tachycardia (VT) was based on either a clockwise ( $n=8)$ or a counterclockwise $(n=3)$ circulating wave front around the central anatomical obstacle. VTs were longlasting and stable with a variation in cycle length of less than $2 \mathrm{~ms}$. The cycle length of the $11 \mathrm{VTs}$ ranged from 153 to $245 \mathrm{~ms}$ with a mean of $180 \pm 26$ $\mathrm{ms}$ (Table 9.1). During VT the conduction velocity in different segments of the ring was not the same. ${ }^{10}$ In the corridor between the LAD and the central obstacle propagation was slow $(22 \pm 4 \mathrm{~cm} / \mathrm{s}, \mathrm{n}=11)$ due to conduction transverse to the epicardial fiber orientation. At the base and the free wall the impulse travelled parallel to the fiber direction with a velocity of about $78 \pm 8 \mathrm{~cm} / \mathrm{s}(\mathrm{n}=11)$. The cycle length of VT was determined mainly by the size of the central obstacle and the length of the area of slow conduction. In VTs with a long cycle length the segment of slow conduction was longer than in VTs with a short cycle length. ${ }^{10}$

During VT the effective refractory period was determined by applying single premature stimuli at the base, the free wall, and the apex of the left ventricle. The maximal spatial difference in refractory periods in 11 VTs was $5 \pm 3 \mathrm{~ms}$ (range 2-12 ms). There was no anatomically defined area in the ventricle with a systematically longer or shorter refractory period. The average refractory period during 11 VTs was $106 \pm 11 \mathrm{~ms}$ with an excitable gap of $74 \pm 17 \mathrm{~ms}$. None of the 11 VTs could be terminated by single premature stimuli.

During VT, entrainment with 10 stimuli was tested at three different pacing sites. At longer pacing intervals VT was transiently entrained but resumed its normal activation pattern after cessation of pacing. Entrainment at short pacing intervals ( $99 \pm 15 \mathrm{~ms}$ ) resulted in termination of VT in 29 of 33 cases. The mechanisms for termination of VT 
Table 9.1:

Characteristics of Reentrant Ventricular Tachycardia

\begin{tabular}{|c|c|c|c|c|c|c|c|c|}
\hline \multirow[b]{2}{*}{ EXP } & \multicolumn{2}{|c|}{ CONTROL } & \multicolumn{2}{|c|}{$\begin{array}{r}\text { ORG7797 } \\
(0.5 \mu \mathrm{M})\end{array}$} & \multicolumn{2}{|c|}{$\begin{array}{l}\text { HEPTANOL } \\
(1.0 \mathrm{mM})\end{array}$} & \multicolumn{2}{|c|}{$\begin{array}{c}\text { d-SOTALOL } \\
(35 \mu \mathrm{M})\end{array}$} \\
\hline & $\mathrm{CL}$ & DWR & $\mathrm{CL}$ & DWR & $\mathrm{CL}$ & DWR & $\mathrm{CL}$ & DWR \\
\hline 1. & 153 & - & 229 & + & & & & \\
\hline 2. & 153 & - & 199 & + & 188 & + & & \\
\hline 3. & 162 & - & 207 & + & 223 & + & & \\
\hline 4. & 168 & - & & & 250 & + & & \\
\hline 5. & 172 & - & 219 & + & 235 & + & & \\
\hline 6. & 175 & - & & & 245 & + & & \\
\hline 7. & 179 & - & 225 & + & & & & \\
\hline 8. & 186 & + & & & & & 196 & - \\
\hline 9. & 189 & + & & & & & 197 & NS \\
\hline 10. & 201 & + & & & & & 212 & NS \\
\hline 11. & 245 & + & & & & & 263 & - \\
\hline AVG & 180 & & 216 & & 228 & & 217 & \\
\hline SD & \pm 26 & & \pm 11 & & \pm 16 & & \pm 27 & \\
\hline
\end{tabular}

Exp $=$ experiment $C L=$ cycle length $D W R=$ sustained double-wave reentry; $A V G=$ average; $S D=$ standard deviation; NS= non-sustained.

by entrainment have been previously described. ${ }^{11}$ In 4 of 33 cases ( 4 hearts) entrainment accelerated VT from $205 \pm 24$ to $115 \pm 14 \mathrm{~ms}$. Mapping of the accelerated VT showed two excitation waves travelling simultaneously in the same direction around the central obstacle (not shown). Due to depression of conduction velocity of the two circulating waves during the accelerated VT, the cycle length of double-wave reentry was always slightly longer than half the cycle length during single-wave reentry.

\section{Effects of Org7797 and Heptanol on VT}

In 7 hearts in which VT could not be accelerated during control, $0.5 \mu \mathrm{M}$ of the experimental class Ic drug ORG7797 and $1.0 \mathrm{mM}$ of Heptanol were administered. Figure 9.1 shows a representative example of the effects of Org7797 and Heptanol on VT. During control the impulse circulated clockwise around the central obstacle with a cycle length of $172 \mathrm{~ms}$. Longitudinal $\left(\theta_{\mathrm{L}}\right)$ and transverse $\left(\theta_{\mathrm{T}}\right)$ conduction velocity were 72.0 and $21.6 \mathrm{~cm} / \mathrm{s}$ respectively with an anisotropy ratio $\left(\theta_{\mathrm{L}} / \theta_{\mathrm{T}}\right)$ of 3.3 . Both Org 7797 and Heptanol slowed VT, increasing the VT interval to 219 and $235 \mathrm{~ms}$. Org7797 equally 

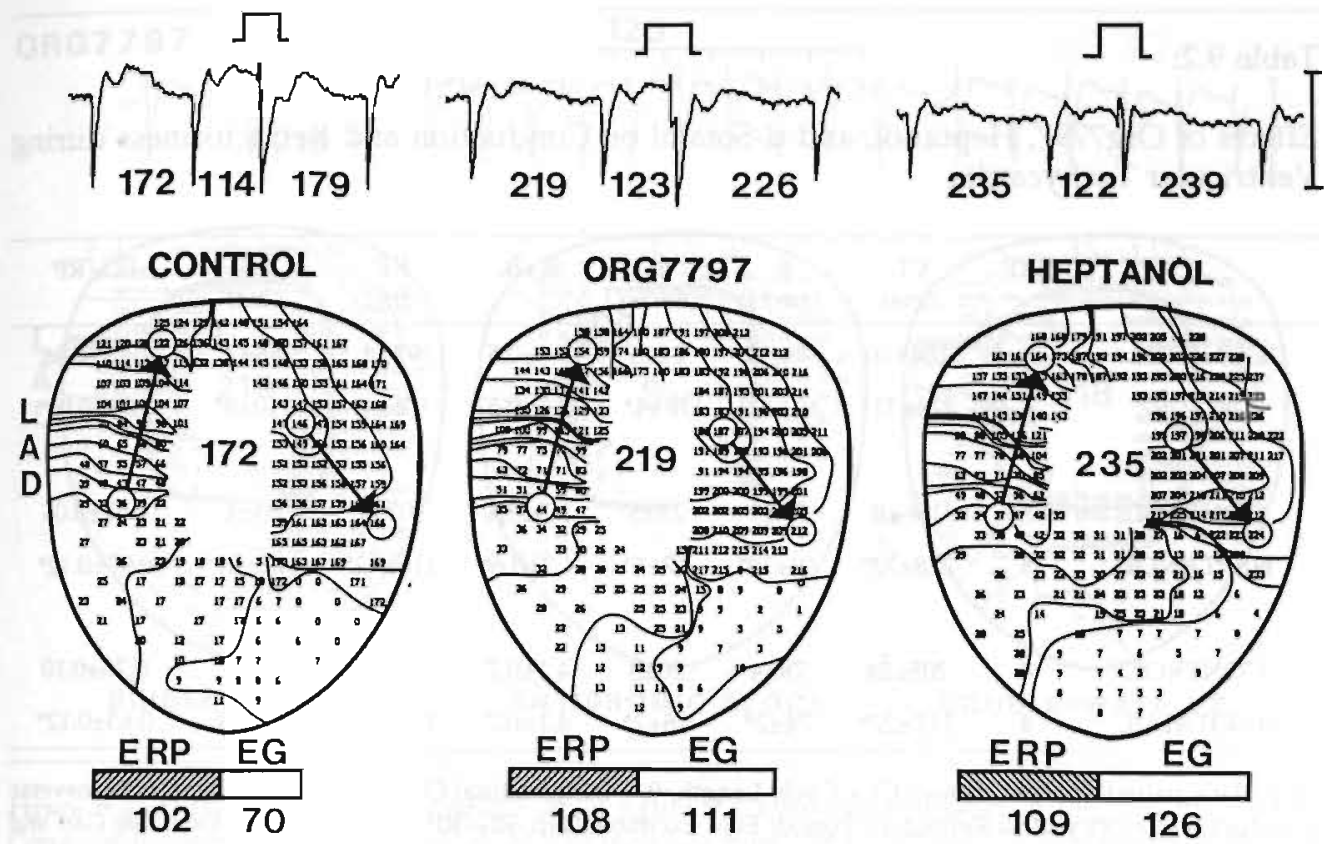

Figure 9.1: The effects of Org7797 and Heptanol on a clockwise VT. The cycle length increased from $172 \mathrm{~ms}$ during control (left panel) to $219 \mathrm{~ms}$ during Org7797 (middle panel) and to $235 \mathrm{~ms}$ during Heptanol (right panel). This was due to a depression of both longitudinal and transverse conduction. The two arrows indicate the sites around the ring at which longitudinal and transverse conduction velocities were measured. The electrograms at the top show the earliest premature stimulus eliciting a propagated response. Brs below the maps show the effective refractory period (ERP) and excitable gap (EG) during VT, measured as the average value at three different sites around the ring. Activation times are given in milliseconds and isochrones are drawn at $10 \mathrm{~ms}$ intervals. Calibration bar $=5$ millivolt.

depressed $\theta_{\mathrm{L}}$ and $\theta_{\mathrm{T}}$ to 57.6 and $16.9 \mathrm{~cm} / \mathrm{s}$ respectively (anisotropy ratio 3.4 ). In contrast, Heptanol depressed $\theta_{\mathrm{T}}$ more than $\theta_{\mathrm{L}}$ increasing the anisotropy ratio to 3.7. During heptanol $\theta_{\mathrm{L}}$ was 53.4 while $\theta_{\mathrm{T}}$ was $14.6 \mathrm{~cm} / \mathrm{s}$. The electrograms above the activation maps show the earliest stimuli which did reset VT both during control and administration of Org7797 and Heptanol. As can be seen, the shortest possible interval induced by premature stimulation prolonged only slightly during Org7797 and Heptanol from 114 to 123 and $122 \mathrm{~ms}$. The bars below the maps show the effects on the effective refractory period (ERP) and the excitable gap (EG) during tachycardia. Org7797 and Heptanol increased the EG from $70 \mathrm{~ms}$ to respectively 111 and $126 \mathrm{~ms}$. Table 9.2 gives the average effects of Org7797 and Heptanol for all experiments. Infusion of Org7797 ( $n=5)$ prolonged the cycle length of VT from 164 to $216(p<0.001)$. This was due to an equal decrease in longitudinal $\left(\theta_{\mathrm{L}}\right)$ and transverse $\left(\theta_{\mathrm{T}}\right)$ conduction velocity by $26 \%$ $(\mathrm{p}<0.05)$. In contrast to the cycle length of VT, the refractory period at three pacing sites was only slightly prolonged from 97 to $106 \mathrm{~ms}(\mathrm{p}<0.05)$. As a result, Org7797 considerably enlarged the excitable gap during VT from 67 to $110 \mathrm{~ms}(\mathrm{p}<0.001)$. The ratio between the excitable gap and the refractory period increased from 0.68 to 1.03 
Table 9.2:

Effects of Org7797, Heptanol, and d-Sotalol on Conduction and Refractoriness during Ventricular Tachycardia.

\begin{tabular}{|c|c|c|c|c|c|c|c|c|}
\hline & EXP & $\begin{array}{c}\mathrm{CL} \\
(\mathrm{ms})\end{array}$ & $\begin{array}{c}\theta_{\mathrm{L}} \\
(\mathrm{cm} / \mathrm{s})\end{array}$ & $\begin{array}{c}\theta_{\tau} \\
(\mathrm{cm} / \mathrm{s})\end{array}$ & $\theta_{\mathrm{L}} / \theta_{\tau}$ & $\begin{array}{c}\mathrm{RP} \\
(\mathrm{ms})\end{array}$ & $\begin{array}{c}\text { EG } \\
\text { (ms) }\end{array}$ & $\mathrm{EG} / \mathrm{RP}$ \\
\hline CONTROL & 5 & $164 \pm 10$ & $78 \pm 12$ & $24 \pm 5$ & $3.4 \pm 0.5$ & $97 \pm 4$ & $66 \pm 7$ & $0.68 \pm 0.06$ \\
\hline ORG7797 & 5 & $216 \pm 11^{*}$ & $62 \pm 13^{*}$ & $19 \pm 4^{*}$ & $3.3 \pm 0.5$ & $106 \pm 4^{*}$ & $110 \pm 9^{*}$ & $1.03 \pm 0.08^{*}$ \\
\hline CONTROL & 5 & $166 \pm 8$ & $79 \pm 9$ & $23 \pm 5$ & $3.5 \pm 0.4$ & $103 \pm 7$ & $63 \pm 4$ & $0.62 \pm 0.06$ \\
\hline HEPTANOL & 5 & $228 \pm 22 *$ & $69 \pm 10^{*}$ & $17 \pm 4^{*}$ & $4.2 \pm 0.6^{*}$ & $116 \pm 12^{*}$ & $112 \pm 14^{*}$ & $0.97 \pm 0.12^{*}$ \\
\hline CONTROL & 4 & $205 \pm 24$ & $78 \pm 4$ & $20 \pm 3$ & $4.1 \pm 0.7$ & $116 \pm 8$ & $89 \pm 17$ & $0.76 \pm 0.10$ \\
\hline d-SOTALOL & 4 & $217 \pm 27^{*}$ & $74 \pm 2^{*}$ & $18 \pm 2^{*}$ & $4.1 \pm 0.7$ & $133 \pm 11^{*}$ & $84 \pm 19$ & $0.63 \pm 0.12^{*}$ \\
\hline
\end{tabular}

* $=p<0.05$, paired Student's t-test; $C L=C$ cle Length; $\theta_{\mathrm{T}}=$ Longitudinal Conduction Velocity; $\theta_{\mathrm{T}}=$ Transverse Conduction Velocity; RP= Refractory Period; EG= Excitable Gap; EG/RP= Ratio between Excitable Gap and Refractory Period

(p<0.05). Heptanol increased the cycle length of VT from 166 to $228 \mathrm{~ms}(\mathrm{p}<0.001) . \theta_{T}$ was affected more than $\theta_{\mathrm{U}}$, resulting in a significant increase in the anisotropy ratio from 3.5 to $4.2(p<0.05)$. Since the refractory period increased less than the cycle length of VT, administration of heptanol resulted in a considerable prolongation of the excitable gap from 63 to $112 \mathrm{~ms}(\mathrm{p}<0.001)$. The ratio between the refractory period and the excitable gap increased from 0.62 to $0.97(p<0.05)$.

\section{Inducibility of Double-wave Reentry during Org7797 and Heptanol}

Both Org7797 and Heptanol considerably changed the susceptibility to acceleration of VT. In all 7 hearts, either the administration of Org7797 or Heptanol enabled the induction of double-wave reentry (Table 9.1). Figure 9.2 shows the mechanism of acceleration of VT after administration of Org7797 in the same heart as in figure 9.1. Whereas during control rapid pacing did not lead to acceleration of VT (not shown), during Org7797 sustained double-wave reentry was induced by entrainment with a pacing interval of $120 \mathrm{~ms}$. The left map shows the effects of Org7797 on the activation pattern during VT. The circulating wave still travelled in a clockwise direction but compared to control (figure 9.1) conduction velocity was depressed in all segments of the circuit. In the segment between the LAD and the central obstacle at the outer edge of the ring, a local inhomogeneity in conduction occurred from the 60 to the $110 \mathrm{~ms}$ isochrone over a distance of $4.5 \mathrm{~mm}$. During the first stimuli the normal pattern of entrainment was present (not shown). However, during S10 (middle map) the paced antidromic wave was blocked ( $t=49 \mathrm{~ms}$ ) in the corridor between the LAD and the obstacle. Due to slow conduction, the orthodromic wave of $S 9$ did not arrive before $\mathrm{t}=140 \mathrm{~ms}$ at the site where block had occurred during $\mathrm{S10}$ (right map). The $91 \mathrm{~ms}$ that 


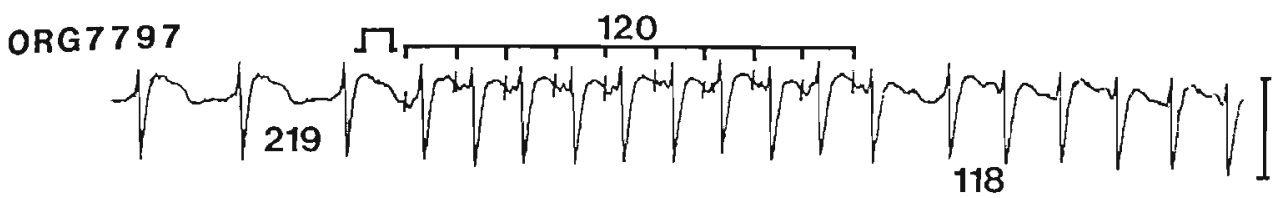

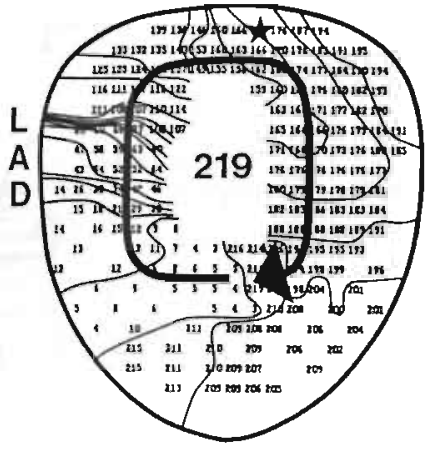

SINGLE-WAVE VT

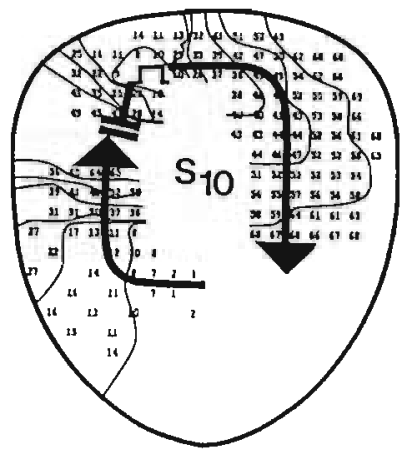

ANTIDROMIC BLOCK

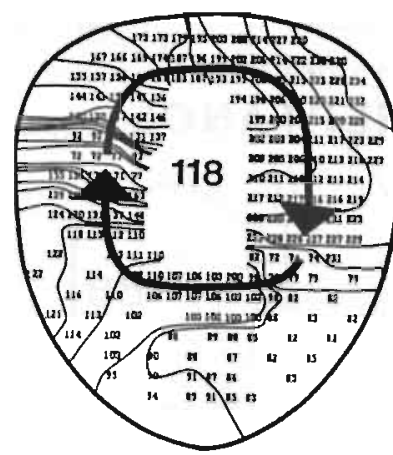

DOUBLE-WAVE VT

Figure 9.2: Entrainment of the same VT as in figure 9.1 after administration of Org7797 (0.5 mM). Compared to control the cycle length of VT had increased from 172 to $219 \mathrm{~ms}$. The electrogram at the top shows that entrainment at 120 ms interval resulted in acceleration of VT. In the left panel the activation map during VT is given. The map in the middle shows the activation pattern during the last entrained beat during which the paced antidromic wave front was blocked (double bar). The right map shows the resulting initiation of double-wave reentry. In the middle and right map the moment of stimulation is taken as $t=0$. Activation times are given in milliseconds and isochrones are drawn at $10 \mathrm{~ms}$ interval. Arrons indicate the direction of the several wave fronts. The double bar indicates conduction block. Calibration bar $=5$ milliVolts. See text for description.

elapsed between antidromic block of S10 and arrival of the previous orthodromic wave of S9 was sufficient for recovery of excitability at the site of block and double-wave reentry was initiated. Due to the short and only partially excitable gap during doublewave reentry, the revolution time of each of the two waves was prolonged to $236 \mathrm{~ms}$ resulting in a cycle length of $118 \mathrm{~ms}$.

In figure 9.3 a similar example is given of acceleration of VT by entrainment after administration of heptanol. During control, VT had a cycle length of $153 \mathrm{~ms}$ and was terminated by rapid pacing at an interval of $100 \mathrm{~ms}$ (top electrogram). During Heptanol the cycle length of clockwise VT increased to $188 \mathrm{~ms}$ (upper left map). Entrainment with an interval of $100 \mathrm{~ms}$ now resulted in acceleration of VT from 188 to $115 \mathrm{~ms}$ (lower electrogram). During the first entrained beats the normal pattern of collision and reset of VT occurred (not shown). The maps show the changes in activation pattern associated with acceleration of VT. During $\$ 9$ conduction block of the antidromic wave occurred at $t=26 \mathrm{~ms}$ close to the site of pacing (upper right map). The orthodromic wave of $\$ 8$ could now proceed unopposed and collided with the antidromic wave of S10 (lower left map). As a result, after cessation of pacing the orthodromic waves of both S9 and S10 could continue to circulate around the central obstacle, leading to sustained double-wave reentry (lower right map). 
CONTROL

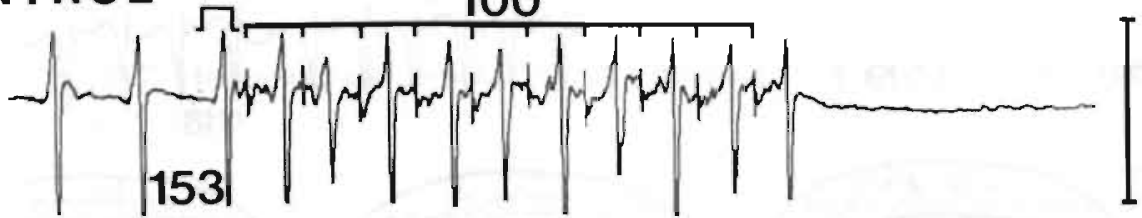

HEPTANOL $几, 100$
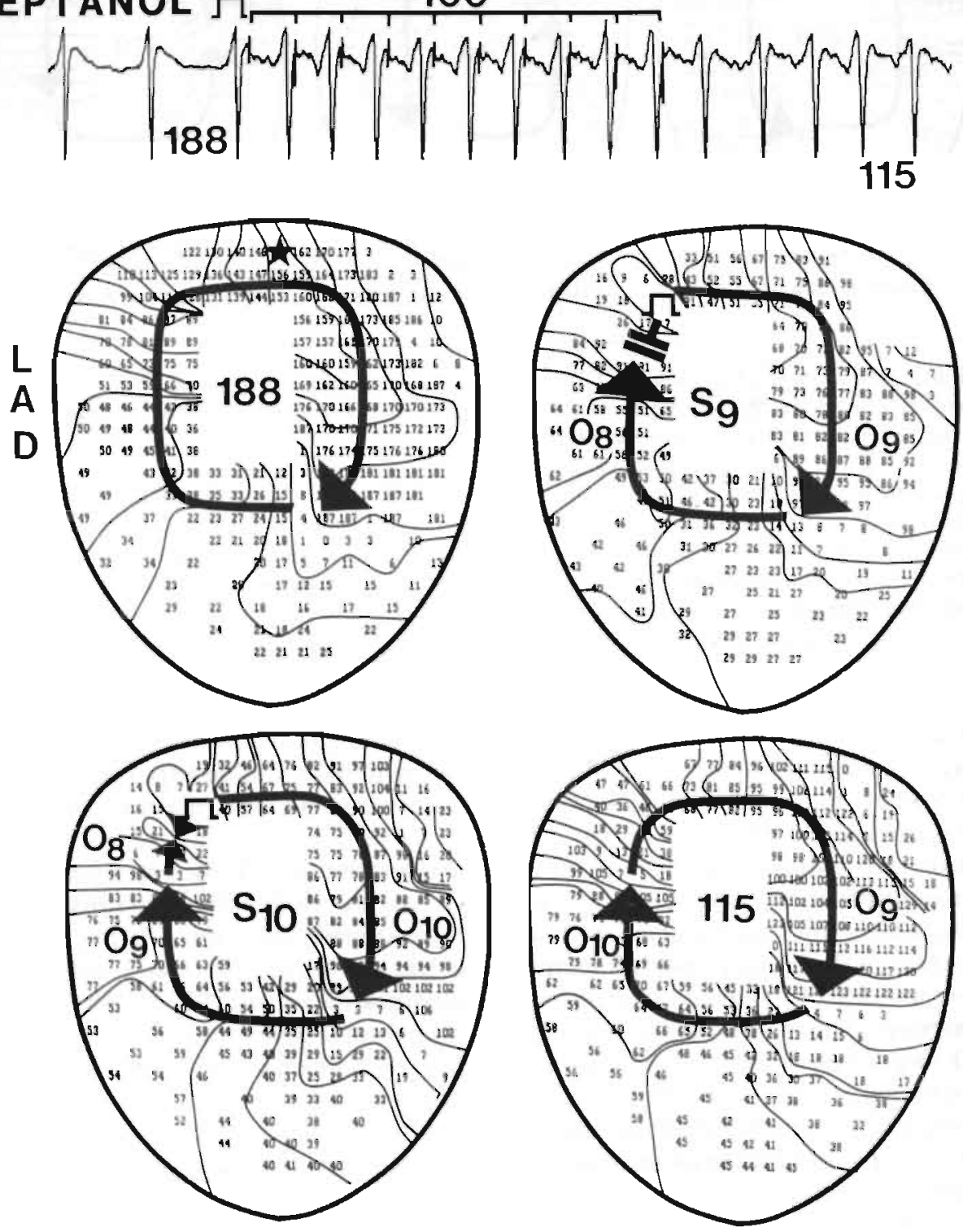
HEPTANOL WASH-OUT
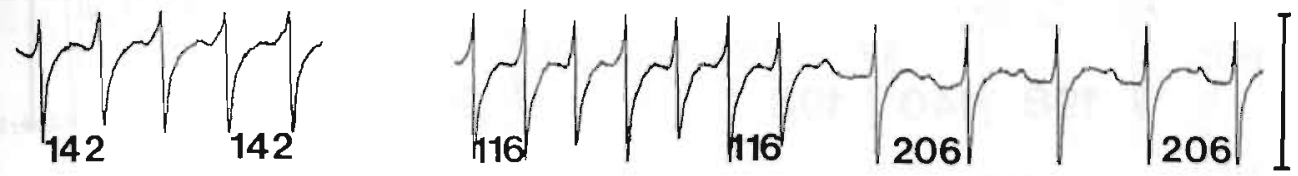

Double-Wave Reentry

\section{Single-Wave Reentry}
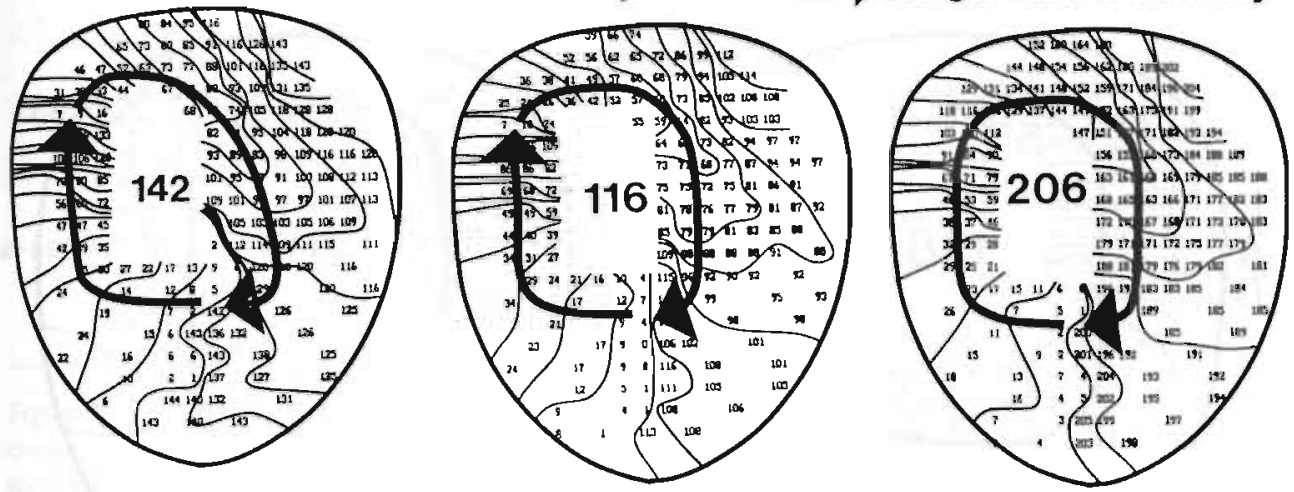

Figure 9.4: Conversion of double-wave reentry to single-wave tachycardia during wash-out of Heptanol. The left map and the electrogram at the top show the activation of double-wave tachycardia with an interol of $142 \mathrm{~ms}$ during Heptanol. During wash-out, double-wave VT first accelerated to $116 \mathrm{~ms}$ (middle panel). Suddenly, after three minutes of wash-out the fast VT spontaneously converted to a slower VT with an interval of $206 \mathrm{~ms}$ based on a single circulating wave around the obstacle (right panel). Activation times are given in milliseconds and isochrones are drawn at $10 \mathrm{~ms}$ interval. Arrows indicate the direction of the circulating waves. Calibration bar $=5$ milliVolt.

In one experiment Heptanol was washed out after sustained double-wave reentry had been induced. During heptanol administration the double-wave VT had a cycle length of $142 \mathrm{~ms}$ (figure 9.4, left). After the infusion of heptanol was stopped, first the VT accelerated to $116 \mathrm{~ms}$ (middle panel). This was due to the reversibility of the uncoupling effect of heptanol, resulting in a restoration of the original conduction velocity. After about three minutes, suddenly the rate of tachycardia markedly decreased and the cycle length of VT increased from one beat to the next from 116 to

Figure 9.3 (left): The electrograms at the top show termination of VT by rapid pacing during control and acceleration of VT after administration of Heptanol. The bar below the electrograms indicates the refractory period (RP) and the excitable gap (EG) of VT during Heptanol. The four maps shows the transition from single-wave (upper left) to double-wave reentry (lower right). During $\$ 9$ (upper right) antidromic block occurred close to the pacing site, resulting in two circulating orthodromic waves. The lower left map shows entrainment of double-wave reentry by S10. After cessation of pacing sustained double-wave reentry with a cycle length of $116 \mathrm{~ms}$ became apparent (lower right map). In the upper right and lower left map the moment of stimulation is taken as $t=0$. Activation times are given in milliseconds and isochrone lines are draum at $10 \mathrm{~ms}$ interval. Arrozos indicate the direction of the several wave fronts. The double bar indicates conduction block. Calibration bar $=5$ millivolts. 


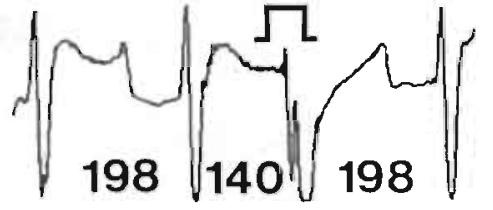

CONTROL

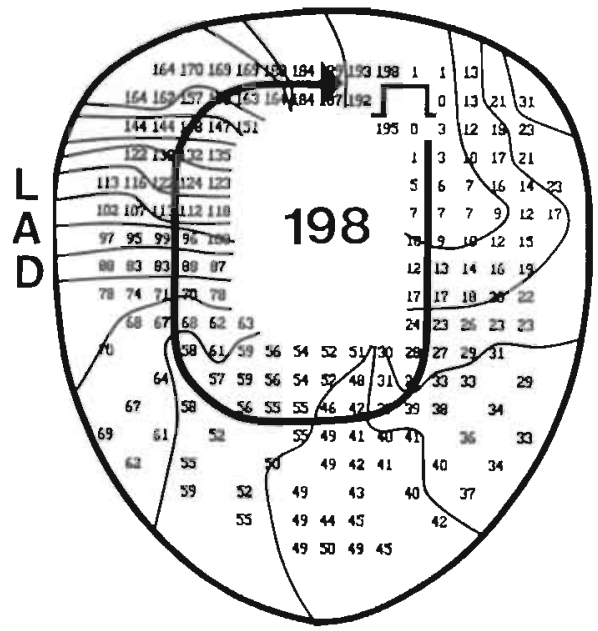

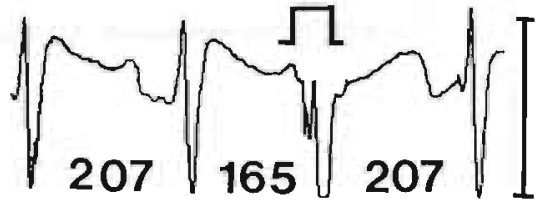

d-SOTALOL

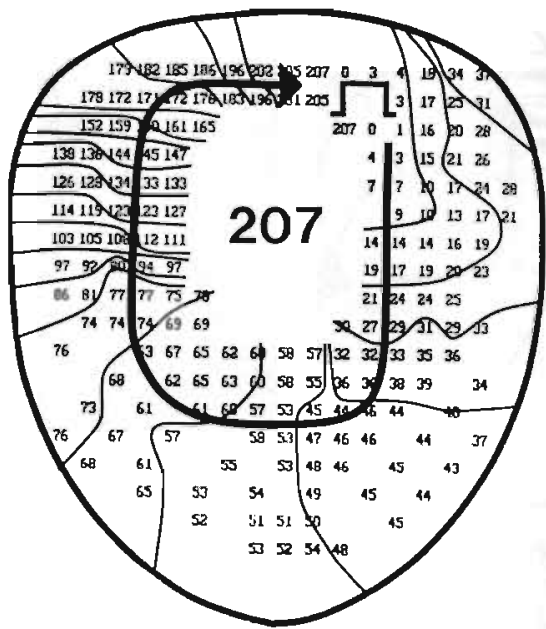

Figure 9.5: The effects of a single premature stimulus on VT during control and d-Sotalol. $d$-Sotalol increased the cycle length of VT from 198 to $207 \mathrm{~ms}$. Premature beats with coupling intervals up to 140 (control) and $165 \mathrm{~ms}$ (d-Sotalol) were conducted with the same velocity as the circulating wave during VT. The map show the activation of the paced orthodromic wavefront. The revolution time of the paced impulses was the same as during VT. Thus, both during control and d-Sotalol a large fully excitable gap was present. Activation times are given in milliseconds and isochrones are drawn at $10 \mathrm{~ms}$ intervals. Calibration bar $=5$ milliVolt.

$206 \mathrm{~ms}$. As can be seen from the activation maps this was caused by the disappearance of one of the two circulating waves, converting the fast double-wave tachycardia into a slower single-wave reentry.

\section{Termination of Double-Wave Reentry by d-Sotalol}

In the 4 hearts in which during control VT could be accelerated, d-Sotalol $(35 \mu \mathrm{M})$ was administered. The cycle length of VT increased only slightly from 205 to $217 \mathrm{~ms}$ $(\mathrm{p}<0.05) . \theta_{\mathrm{L}}$ and $\theta_{\mathrm{T}}$ were equally affected and the ratio of anisotropy $\left(\theta_{\mathrm{L}} / \theta_{\mathrm{T}}\right)$ was not changed. The refractory period during VT was increased by $15 \%$ from 116 to $133 \mathrm{~ms}$ $(\mathrm{p}<0.05)$. As a result, although the excitable gap shortened only slightly from 89 to 84 ms (NS, $p=0.07$ ), the ratio between the excitable gap and the refractory period decreased significantly from 0.76 to 0.63 ( $p<0.05$ ) (see Table 9.2).

The decrease in conduction velocity during VT by d-Sotalol could not be explained by shortening of the excitable gap. This is illustrated in figure 9.5. In this example, the cycle length of a clockwise VT had been slightly prolonged by d-Sotalol from 198 to 207 


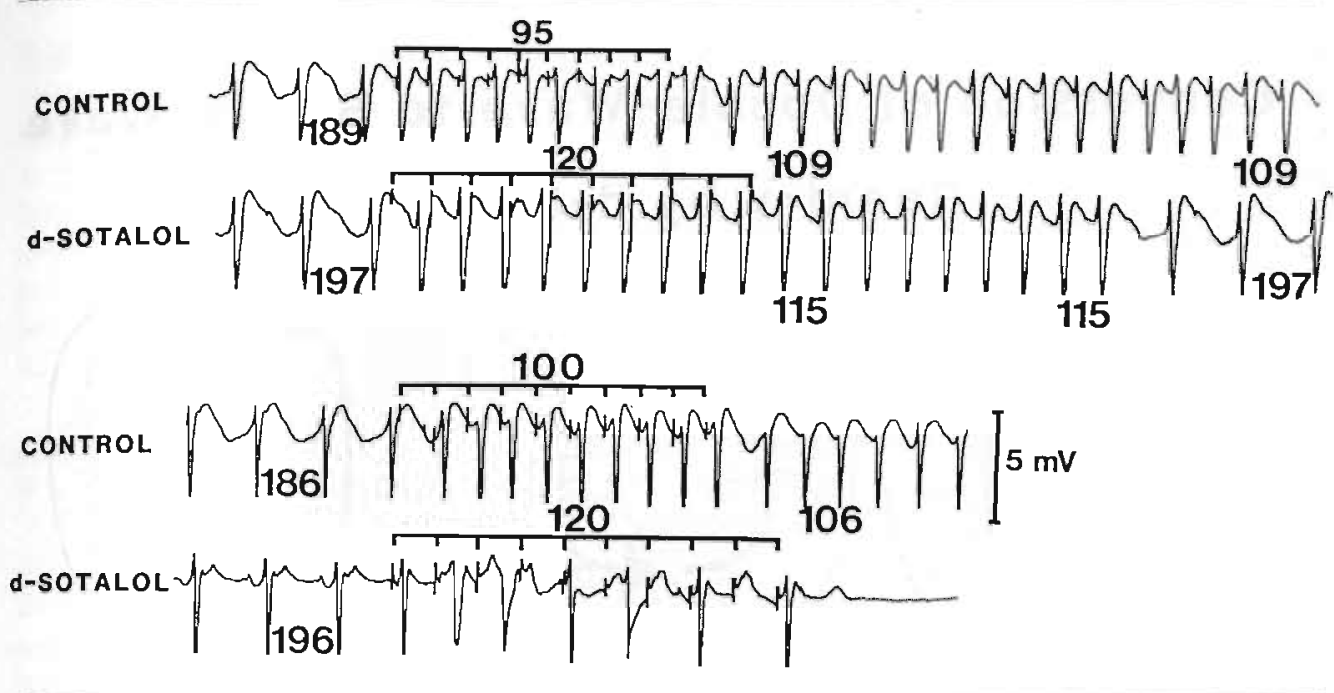

Figure 9.6: Effects of d-Sotalol on inducibility and stability of double-wave reentry. Top: During control entrainment at $95 \mathrm{~ms}$ intercal resulted in sustained acceleration of VT from 189 to $109 \mathrm{~ms}$. During d-Sotalol acceleration of VT was no longer sustained and after 8 cycles VT converted to its original rate. Bottom: During control, entrainment at $100 \mathrm{~ms}$ interval resulted in sustained acceleration of VT from 186 to $106 \mathrm{~ms}$. After administration of $d$-Sotalol rapid pacing did no longer result in acceleration of VT.

ms. During control, premature beats up to a coupling interval of $140 \mathrm{~ms}$ were conducted without delay with the same revolution time around the ring as the VT cycle length of $198 \mathrm{~ms}$. During d-Sotalol administration, premature beats up to a coupling interval of $165 \mathrm{~ms}$ conducted with the same velocity as during VT with a revolution time of $207 \mathrm{~ms}$. This demonstrates that also during d-Sotalol still a large fully excitable gap was present during VT. Thus, the slowing of conduction during VT could not be explained by propagation of the circulating wave through partially refractory tissue. During d-Sotalol, entrainment could still terminate VT in all hearts. However, the inducibility and stability of double-wave reentry was markedly affected by d-Sotalol (Table 9.1). In 2 hearts entrainment could still induce double-wave reentry but the accelerated VT was no longer sustained and within a few seconds converted spontaneously to the original VT. In the other 2 hearts, d-Sotalol completely prevented induction of double-wave reentry. Figure 9.6 gives an example of both responses. The upper two tracings show that during control, pacing at an interval of $95 \mathrm{~ms}$ resulted in sustained acceleration of VT from 189 to $109 \mathrm{~ms}$. After d-Sotalol was added VT was slowed to $197 \mathrm{~ms}$ and entrainment only resulted in non-sustained acceleration of VT to $115 \mathrm{~ms}$. The lower two tracings illustrate that after d-Sotalol double-wave reentry could no longer be induced.

In 2 hearts, infusion of d-Sotalol was started after sustained double-wave reentry had been induced. First, d-Sotalol gradually slowed the accelerated VT from $109 \pm 6$ to 121 $\pm 4 \mathrm{~ms}$ until it was suddenly converted to single wave reentry with a cycle length of 213 $\pm 9 \mathrm{~ms}$. Figure 9.7 shows three consecutive maps and the associated electrograms around the circuit during conversion of double-wave to single-wave reentry by d-Sotalol. Prior to termination of double-wave reentry with a cycle length of $118 \mathrm{~ms}$, the revolution 


\section{Conversion of Double-Wave to Single-Wave}

\section{Reentry by d-Sotalol}

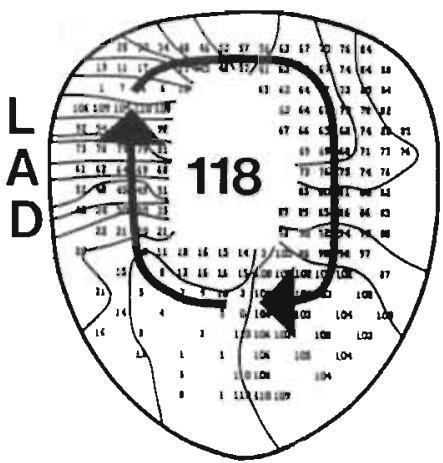

DOUBLE-WAVE

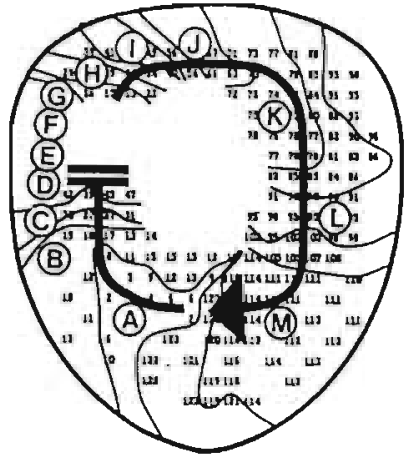

BLOCK

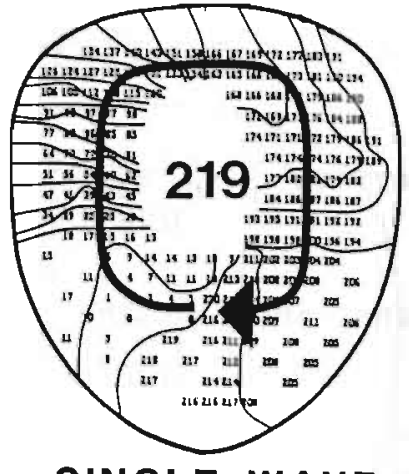

SINGLE-WAVE

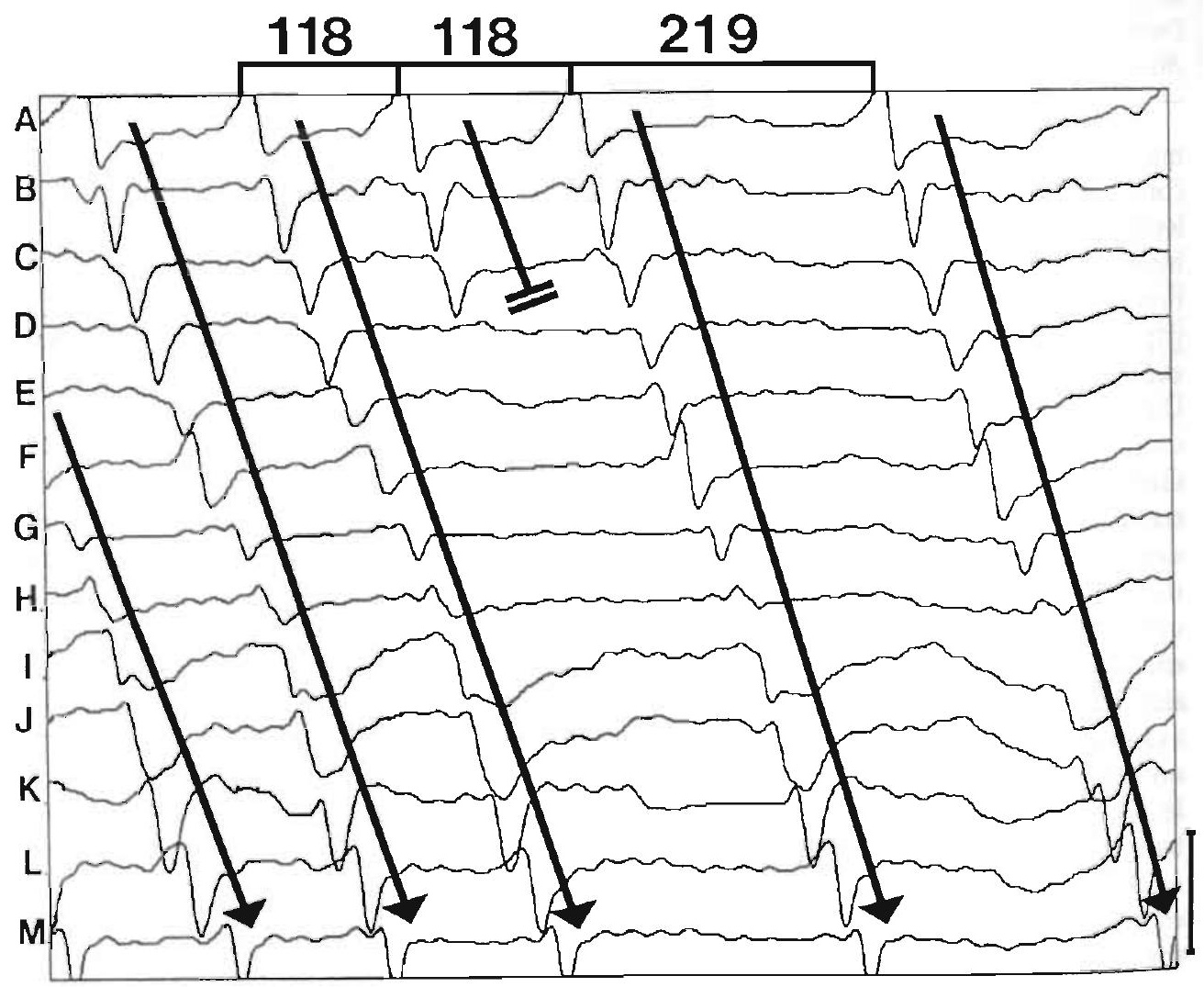


time of the two circulating waves was $236 \mathrm{~ms}$ (left map). Suddenly one of the circulating waves was blocked between electrodes $C$ and $D$ in the area of slow conduction (middle map). As a result the VT cycle length suddenly prolonged from 118 to $219 \mathrm{~ms}$ (right map). After the sudden disappearance of one of the two waves, the revolution time of the remaining wave decreased from 236 to $219 \mathrm{~ms}$. This increase in conduction velocity can be explained by the larger excitable gap during single-wave reentry. Obviously, during double-wave reentry only a partially excitable gap existed between the two circulating waves, decreasing the conduction velocity of both wave fronts.

\section{Importance of the Pacing Site for Induction of Double-Wave Reentry}

In all 11 hearts, entrainment was performed at three different pacing sites around the circuit, located at the base, the free wall, and the apex of the left ventricle. Both during control and during administration of Heptanol, Org7797, and d-Sotalol VT could be terminated by conduction block of one of the paced orthodromic waves during entrainment. In $85 \%$ of all cases of termination of VT, the site of orthodromic block was located in the corridor between the LAD and the central obstacle. Previously, we have demonstrated that conduction block preferentially occurred in a segment of slow transverse conduction. ${ }^{11}$ During rapid pacing from all the three sites tested the paced orthodromic wave propagated through at least part of the segment of slow transverse conduction. Consequently, VT could be terminated from all sites around the circuit and there was no preferential pacing site for termination.

In all cases of induction of double-wave reentry, both during control and after administration of drugs, one of the paced antidromic wave fronts was blocked. Since conduction block occurred preferentially in the segment of slow conduction, antidromic block and double-wave reentry could only be induced by pacing at a site distal to the segment of slow conduction. Thus, during clockwise VT double-wave reentry could only be induced by pacing at the base, while during counterclockwise VT initiation of double-wave reentry only occurred during entrainment at the apex.

\section{DISCUSSION}

\section{Antiarrhythmic effects of heptanol, Org7797, and d-Sotalol}

Antiarrhythmic drugs can be effective either by terminating VT, slowing its rate, or by lowering the inducibility of VT. In our study the experimental class Ic drug Org7797 and the uncoupling agent heptanol both slowed VT by about $35 \%$, whereas d-Sotalol only slightly slowed VT by $6 \%$. Spinelli and Hoffman ${ }^{12}$ have shown that during atrial flutter class I drugs were also more effective than class III drugs in slowing the rate.

Figure 9.7 (left): Conversion of double-wave to single-wave reentry by administration of d-Sotalol ( $35 \mu M)$. The three panels at the top show the activation maps before, during, and after conversion. Electrograms $A-M$ were recorded around the circuit as indicated in the middle map. Prior to termination the cycle length of double-wuve VT was $118 \mathrm{~ms}$ (left map). In the middle map one of the two circulating waves was blocked between electrodes $C$ and $D$ in the corridor between the $L A D$ and the central obstacle. The remaining wave. front continued to circulate with a cycle length of $219 \mathrm{~ms}$ (right map). Activation times are given in milliseconds and isochrone lines are drawn at $10 \mathrm{~ms}$ interial. Arrows indicate the direction of the several wave fronts. The double bar indicates conduction block. Calibration bar $=5$ millivolt. 
The slowing of conduction during VT by d-Sotalol could not be explained by shortening of the excitable gap. The fact that early premature wavefronts could propagate around the circuit without any slowing of conduction strongly suggests that in our model of VT also during administration of a class III drug a fully excitable gap exists. In a previous study, Carmeliet ${ }^{13}$ showed that in guinea-pig papillary muscle Sotalol reduced $\mathrm{V}_{\max }$ at concentrations above $100 \mu \mathrm{M}$. In ventricular trabecular muscle Nakaya et al. ${ }^{14}$ found that already at $30 \mu \mathrm{M}$ Sotalol reduced $\mathrm{V}_{\max }$ with $10 \%$. We therefore assume that in our preparation the slowing of conduction velocity by d-Sotalol may have been due to a slight class I effect.

Both class I and class III drugs at low concentrations have been shown to be very succesfull in termination of experimental atrial flutter. ${ }^{12}$ In our experiments neither heptanol, ORG7797, or d-Sotalol were able to terminate VT. Also application of premature stimuli under these conditions failed to terminate VT. This indicates that in our model of reentrant VT, both during control and during antiarrhythmic drugs still a high safety factor for conduction existed in every segment of the circuit. On the other hand, if a reentrant tachycardia can be terminated by antiarrhythmic drugs this may implicate that at least in some part of the circuit a low safety factor for conduction must exist.

\section{Effects of drugs on the inducibility of double-wave reentry}

For the induction of double-wave reentry the following three prerequisites have to be fulfilled. First, one of the stimulated antidromic wave fronts during entrainment must be blocked. Second, the dimensions of the circuit should be such that the orhodromic wave of the previous stimulus arrives late enough to allow recovery of excitability and reentry of the site of block. Third, the ratio between the excitable gap and the refractory period should be large enough for double-wave reentry to become sustained. During control, acceleration of VT only occurred in the 4 hearts with the longest cycle length and the largest excitable gap. In these cases the refractory period still exceeded the excitable gap. However, during entrainment a rate-dependent shortening of the refractory period must have increased the ratio between the excitable gap and the refractory period above 1 , thus providing enough space for a second wave to circulate. Since the revolution time of each circulating wave was longer than during single-wave reentry, only a partially excitable gap is present during double-wave reentry.

Both Org7797 and Heptanol decreased the conduction velocity more than they prolonged refractoriness. The resulting shortening of the wavelength is considered to be proarrhythmic since it promotes the occurrence of reentry. ${ }^{15,16}$ In this respect, $d$ Sotalol was antiarrhythmic since it prolonged the wavelength by increasing the refractory period more than depressing the conduction velocity. This is in agreement with our present findings that Org7797 and Heptanol both facilitated acceleration of VT while d-Sotalol prevented induction of sustained double-wave reentry. In addition, both administration of d-Sotalol and wash-out of Heptanol terminated double-wave reentry. The critical factor that determines whether a reentrant circuit can sustain two waves is the ratio between the excitable gap and the refractory period. Both Org7797 and Heptanol increased the excitable gap to such extent that it equalled the refractory period. On the other hand d-Sotalol decreased the ratio between the excitable gap and refractory period and in 2 of 4 hearts double-wave reentry was no longer inducible. In the other 2 hearts, double-wave reentry was no longer sustained. Probably, after cessation of pacing the reduction in rate prolonged the refractory period again and the 
excitable gap closed so that the circuit could no longer sustain double-wave reentry.

\section{Feasibility of functional double-wave reentrant VT}

Our results show that an anatomic reentrant pathway with a large excitable gap is a suitable substrate for double-wave reentry. The question arises whether also functionally determined reentry can be accelerated by induction of a second circulating wavefront. As shown in the present study, double-wave reentry can only be induced when there is a ratio of 1 between the excitable gap and the refractory period during VT. During functionally determined reentry generally there is no or only a small partially excitable gap., ${ }^{2,3,17-21}$ In uniform anisotropic myocardium, during functional reentry the excitable gap was found to be $27 \%$ of the VT interval, with a ratio between the excitable gap and the refractory period of not more than $0.37 .{ }^{20}$ In an experimental model of canine myocardial infarction, several investigators have demonstrated that functional reentrant circuits in the epicardial border zone of the infarct can be reset and entrained, demonstrating the presence of an excitable gap. ${ }^{2,3,8}$ In some of these circuits part of the excitable gap was fully excitable since stimulated wavefronts propagated around the circuit with the same revolution time as the circulating wave during VT, ${ }^{2,8}$ The actual size of the excitable gap was not determined but seemed to be no more than $25 \%$ of the VT cycle length. Thus, despite the fact that recently Dillon et al. ${ }^{8}$ observed an isolated case of double-wave reentry during functional VT after canine myocardial infarction, it seems unlikely that double-wave reentry plays an important role in functionally determined reentry. So far, during our experiments on functional reentry ${ }^{17,18,20}$ it has never been observed. It is still unclear whether some anti-arrhythmic agents (class Ic drugs) may enlarge the excitable gap of functional reentry, thereby enhancing the chance that double-wave reentry might occur.

\section{Clinical Implications}

The significance of double-wave reentry as a mechanism of acceleration of VT after myocardial infarction in patients, either in the presence or absence of antiarrhythmic drugs, is unclear. In a study on the mechanisms of acceleration of clinical VT, Brugada et al. ${ }^{5}$ speculated that in 2 of 22 patients the criteria for double-wave reentry might possibly have been fulfilled. However, systematic clinical studies on the inducibility of double-wave reentry at several pacing sites have not yet been performed. Our present results indicate that the induction of double-wave reentry highly depends on the localization of the pacing site relative to the area of slow conduction. Therefore, the possibility of the reentrant substrate to accomodate two circulating waves may be seriously underestimated if only one pacing site is being used. In addition, one should be aware that anti-arrhythmic drugs which widen the excitable gap of a VT may enhance the susceptibility to acceleration of VT by double-wave reentry. 


\section{CONDENSED ABSTRACT}

In 11 Langendorff perfused rabbit hearts, rings of anisotropic left ventricular myocardium were created by a cryoprocedure. Rapid pacing induced reentrant ventricular tachycardia around the ring. During control, entrainment at high rates could terminate VT in all hearts. In four hearts with a large excitable gap, rapid pacing could also accelerate VT by induction of double-wave reentry. In the 7 hearts that could not be accelerated, Org7797 (experimental class Ic) and Heptanol (electrical uncoupler) prolonged the cycle length of VT and increased the ratio between the excitable gap and the refractory period. Double-wave reentry was now inducible in all hearts. d-Sotalol (class III) decreased the ratio between the excitable gap and the refractory period, which either rendered double-wave reentry non-sustained or prevented acceleration of VT. 


\section{REFERENCES}

1. Podrid P, Lampert S, Graboys T, Blatt C, Lown B: Aggravation of arrhythmia by anti-arrhythmic drugs: Incidence and predictors. Am J Cardiol 1987;59:38E-44E

2. Waldecker B, Brugada P, Zehender M, Stevenson W, Den Dulk K, Wellens HJj: Modes of electrical termination of ventricular tachycardia: Importance for selection of implantable antitachycardia devices. Am J Cardiol 1986;57:150-155

3. El-Sherif N, Gough W, Restivo M: Reentrant ventricular arrhythmias in the late myocardial infarction period: 14. Mechanisms of resetting, entrainment, or acceleration of reentrant tachycardia by programmed electrical stimulation. PACE 1987;10:341-371

4. Brugada J, Boersma L, Kirchhof C, Brugada P, Havenith M, Wellens HJJ, Allessie MA: Doublewave reentry as a mechanism of acceleration of ventricular tachycardia. Circulation 1990;81:16331643

5. Brugada J, Brugada P, Boersma L, Mont L, Kirchhof C, Wellens HJJ, Allessie MA: On the mechanisms of ventricular tachycardia acceleration during programmed electrical stimulation. Circulation 1991;83:1621-1629

6. Brugada J, Boersma L, Kirchhof C, Allessie M: Proarrhythmic effects of flecainide. Experimental evidence for increased susceptibility to reentrant arrhythmias. Circulation 1991;84:1808-1818

7. Fitzgerald D, Friday K, Yeung-Lai-Wah J, Bowman A, Lazzarra R, Jackman W: Myocardial regions of slow conduction participating in the reentrant circuit of multiple ventricular tachycardias: Report on ten patients. J Cardiovasc Electrophysiol 1991;2:193-206

8. Dillon S, Coromilas J, Waldecker B, Wit A: Effects of overdrive stimulation on functional reentrant circuits causing ventricular tachycardia in the canine heart. Mechanisms for resumption or alteration of tachycardia. J Cardiovasc Electrophys 1993 (in press)

9. Winslow E, Martorana M, Bell P: Electrophysiological effects of Org7797, a new steroidal antiarrhythmic agent: comparison with class Ia, lb, and Ic drugs. J Cardiovasc Pharmacol 1989;14:205212

10. Brugada J, Boersma L, Kirchhof C, Heynen V, Allessie MA: Reentrant excitation around a fixed anatomical obstacle in uniform anisotropic ventricular myocardium. Circulation 1991;84:12961306

11. Boersma L, Brugada J, Kirchhof C, Allessie M: Entrainment of reentrant ventricular tachycardia in anisotropic rings of rabbit myocardium. Mechanisms of termination, change in morphology, and acceleration of VT. Circulation 1993;88:1852-1865

12. Spinelli $W$ and Hoffman B: Mechanisms of termination of reentrant atrial arrhythmias by class I and class III antiarrhythmic agents. Circ Res 1989;65:1565-1579

13. Carmeliet E: Electrophysiologic and voltage clamp analysis of sotalol effects in cardiac muscle and Purkinje Fibers. J Pharmacol Exp Ther 1985;232:3:77-85

14. Nakaya H, Kimura S, Nakao Y, Kanno M: Effects of nipradilol (K-351) on the electrophysiological properties of canine cardiac tissues: comparison with propranolol and sotalol. Eur J Pharmacol 1984;104:335-344

15. Allessie M, Lammers W, Rensma P, Schalij M, Kirchhof C: Determinants of reentry in cardiac muscle, in Zipes D, Rowlands D (eds): Progress in Cardiology. Philadelphia, Lea \& Febiger, 1988, pp 3-15

16. Rensma P, Allessie M, Lammers W, Bonke F, Schalij M: Length of excitation wave and susceptibility to reentrant atrial arrhythmias in normal conscious dogs. Circ Res 1988;62:395-410

17. Allessie M, Schalij MJ, Kirchhof C, Huyberts M, Boersma L, Hollen J: Experimental electrophysiology and arrhythmogenicity: Anisotropy and ventricular tachycardia. Eur Heart J 1989;10E:8-14

18. Schalij M, Lammers W, Rensma, Allessie M: Anisotropic conduction and reentry in perfused epicardium of rabbit left ventricle. Am J Phys 1992;263:H1466-H1478

19. Allessie M, Bonke F, Schopman F: Circus movement in rabbit atrial muscle as a mechanism of tachycardia. III. The "leading circle" concept: A new model of circus movement in cardiac tissue without the involvement of an anatomic obstacle. Circ Res 1077;41:9-18

20. Boersma L, Brugada J, Kirchhof C, Allessie M: Mapping of reset of anatomic and functional reentry in anisotropic ventricular myocardium. Circulation 1994:89:(in press) 


\section{CHAPTER 10}

\section{TERMINATION OF VENTRICULAR TACHYCARDIA} BY CLASS IC AND CLASS III ANTIARRHYTHMIC AGENTS.

In part: Circulation 1992;85:1879-1887 


\section{INTRODUCTION}

Anti-arrhythmic drugs exert their benificial effect either by slowing and terminating cardiac arrhythmias or by depressing their inducibility.' The choice for a particular antiarrhythmic agent depends both on its mode of action and on the type of arrhythmia and its underlying mechanism..$^{2,3}$ In the management of ventricular tachycardia after myocardial infarction, which is based on reentry, ${ }^{46}$ class I and class III drugs or their combination are succesfull in abolishing about $30 \%$ of clinical VTs. ${ }^{1}$ The potency to slow the rate or terminate clinical VTs has been tested for most drugs but the underlying mechanisms are not well known. During termination of VT by various antiarhythmic drugs peculiarities have been observed ${ }^{7}$ of which the mechanisms are unresolved.

To gain more insight in the mechanisms of termination of VT by antiarrhythmic agents, we studied the activation patterns during application of a class Ic (Flecainide) and an experimental class III (RP62719) drug in an experimental model of VT around an anatomical obstacle in Langendorff perfused anisotropic rabbit myocardium. Our results show that Flecainide markedly depressed conduction before termination of VT occurred at a high dosage. RP62719 had a more prominent effect on the refractory period than on the cycle length of VT and terminated 4 of 8 VTs by abolition of the excitable gap. During both drugs termination of VT was either due to complete conduction block of the reentry wave or collision of the circulating wave with a reflected echo-wave.

\section{METHODS}

\section{Preparation}

In 13 Langendorff-perfused rabbit hearts, a thin layer of left ventricular subepicardium was created by a cryoprocedure. Details of the perfusion system and the cryoprocedure have been described in chapter 2 . In the thin epicardial layer, a fixed anatomical obstacle $(25 \times 10 \mathrm{~mm})$ was created (chapter 4$)$.

\section{Recording and Stimulation}

A spoon-shaped mapping electrode, molded to the epicardial surface of the left ventricle, containing 248 individual silver electrodes (diameter $0.3 \mathrm{~mm}$, resolution 2.25 $\mathrm{mm}$ ), was used to map the ventricular activation. The on-line mapping system to record and analyze the data has been described in chapter 3 .

Bipolar stimulation could be performed through any selected pair of electrodes in the electrode spoon. Sustained monomorphic ventricular tachycardia was induced by incremental pacing. During RP62719 the effective refractory period (ERP) during VT was determined at each concentration. The stimulation protocol to measure the ERP has been described in chapter 7 .

\section{Drug infusion}

Flecainide (Tambocor ${ }^{\circledR}, 3 \mathrm{M}$ Riker) and RP62719 (Rhone-Poulenc) were dissolved in Tyrode and added to the perfusion fluid by an infusion pump. The concen-tration concentration of flecainide $(n=5)$ was raised with steps of $1.0 \mathrm{mg} / \mathrm{l}$ until VT was terminated. RP62719 was tested at $0.03(n=8), 0.3(n=4)$, and $3.0 \mu M(n=4)$. At each concentration of both drugs an equilibration time of 15 minutes was allowed before the concentration was raised 


\section{Statistical Analysis}

Results were compared using the paired Student's t-test and ANOVA. P-values of less than 0.05 were taken as statistically significant.

\section{RESULTS}

During control, in thirteen hearts VT was based on either a clockwise ( $n=9)$ or a counterclockwise $(n=4)$ circulating wave around the central obstacle. The average cycle length of the 13 VTs was $166 \pm 26 \mathrm{~ms}$, ranging from 122 to $204 \mathrm{~ms}$. All VTs were very stable with a variability in cycle length of less than $2 \mathrm{~ms}$, and spontaneous termination of VT was never observed.

\section{Effects of Flecainide and RP62719 on VT}

In 5 hearts during VT the concentration of Flecainide was gradually raised in steps of $1 \mathrm{mg} / \mathrm{l}$. Figure 10.1 gives the activation maps during control and 2, 4, and $6 \mathrm{mg} / \mathrm{l}$ of Flecainide. During control (upper left) the clockwise circulating wave propagated around the central obstacle with a cycle length of $164 \mathrm{~ms}$. In the corridor between the LAD and the obstacle slow conduction occurred transverse to the fiber orientation, while at the base and free wall longitudinal conduction was much faster (chapter 4). During control, adjacent electrodes were never separated by more than 2 isochrone lines (apparent conduction velocity $>10 \mathrm{~cm} / \mathrm{s}$ ) indicating that conduction was uniform. When the concen-tration of Flecainide was raised to 2 (upper right), 4 (lower left), and $6 \mathrm{mg} / \mathrm{l}$ (lower right) the cycle lenght of VT increased to 243, 374, and $541 \mathrm{~ms}$ respectively. The activation maps show that at higher concentrations of Flecainide the number of isochrones increased markedly due to a strong depression of conduction. At $6 \mathrm{mg} / \mathrm{l}$ (lower right map) the circulating wave meandered through the ring to find a continuous pathway for conduction. Adjacent electrodes could be separated by more than 5 isochrones, indicating an apparent conduction velocity of less than $5 \mathrm{~cm} / \mathrm{s}$.

The experimental class III drug RP62719 was administered at concentrations of 0.03 $(\mathrm{N}=8), 0.3(\mathrm{~N}=4)$, and $3.0(\mathrm{~N}=4) \mu \mathrm{M}$. An example of the effects of RP62719 on VT is given in figure 10.2. The three panels show the activation maps of VT during control (left), 0.3 (middle), and $3.0 \mu \mathrm{M}$ (right) of RP62719. During control (left map) the circulating wave propagated in a clockwise direction around the obstacle with a revolution time of $190 \mathrm{~ms}$. Slow transverse conduction occurred in the corridor between the LAD and the obstacle while fast longitudinal propagation occurred at the base and free wall. At $0.03 \mu \mathrm{M}$ of RP62719 the activation pattern of VT was not changed (not shown). At $0.3 \mu \mathrm{M}$ of RP62719 the cycle length of VT minimally prolonged to $195 \mathrm{~ms}$ (middle map) and only at $3.0 \mathrm{mM}$ of RP62719 the cycle length of VT notably increased to $236 \mathrm{~ms}$. The right activation map shows that during $3.0 \mu \mathrm{M}$ the conduction velocity was markedly slowed in all segments of the ring but there were no arcs of local conduction block or areas of inexcitability.

Figure 10.3 shows the dose-response curves of Flecainide (dots) and RP62719 (squares). The VT cycle length is plotted on the ordinate while the concentrations of Flecainide and RP62719 are plotted on the abscissa. Flecainide markedly increased the cycle length of VT from $167 \pm 21 \mathrm{~ms}$ during control to $741 \pm 134 \mathrm{~ms}$ at $7 \mathrm{mg} / \mathrm{l}(\mathrm{n}=5)$, resulting in a steep curve. In contrast RP62719 had a less pronounced effect, maximally increasing the VT cycle length from $166 \pm 26 \mathrm{~ms}(\mathrm{n}=8)$ during control to $251 \pm 20(\mathrm{~N}=4)$ at $3.0 \mu \mathrm{M}$. 


\section{Effects of Flecainide on VT}

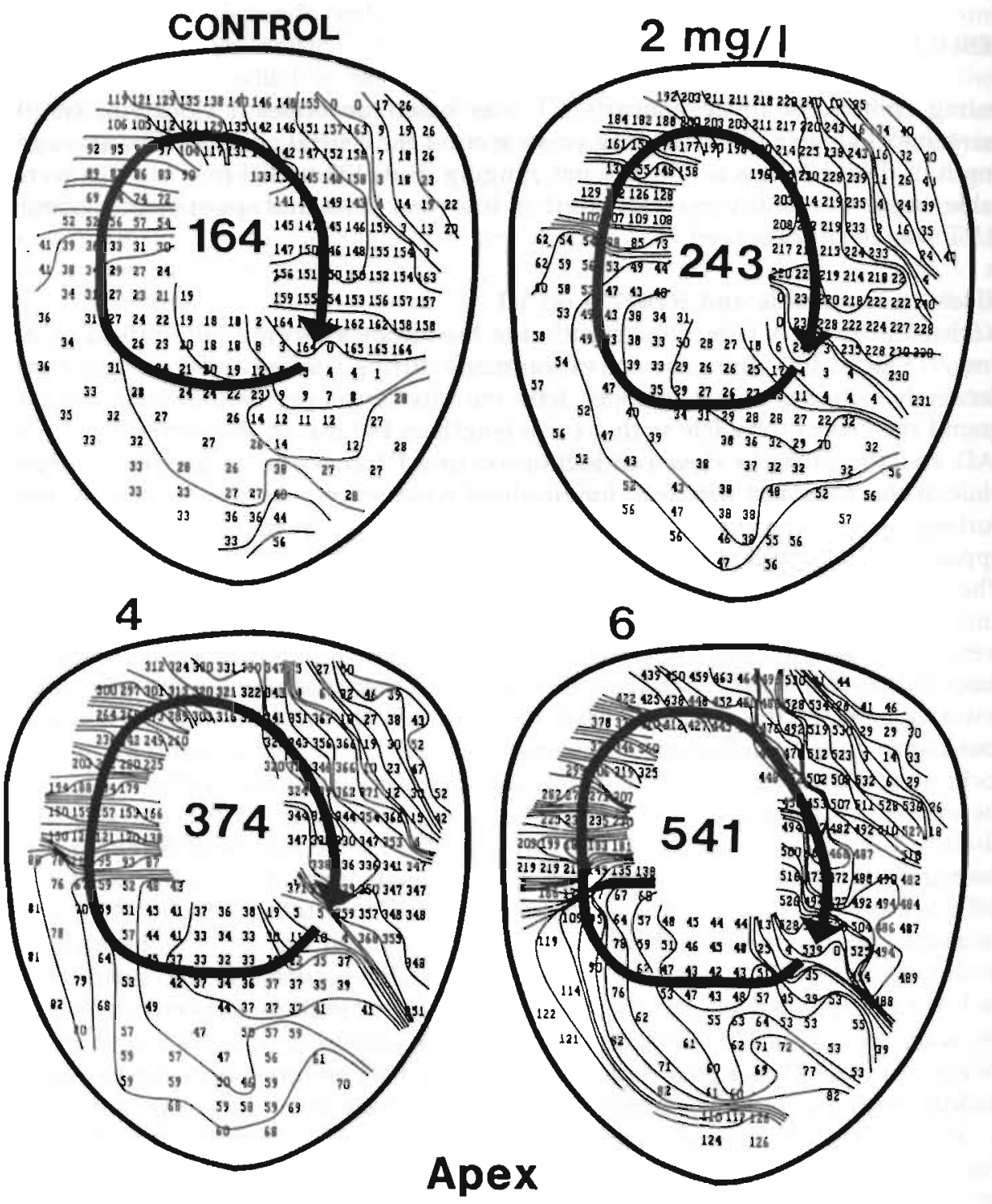

Figure 10.1: Effects of flecainide on reentrant ventricular tachycardia. The four maps shoto the activation pattern of VT during control, 2, 4, and $6 \mathrm{mg} / \mathrm{l}$ of flecainide. During control the clockwise circulating wave propagated around the central obstacle with a cycle length of 164 ms (upper left map). During 2, 4, and 6 mgll of flecainide the VT cycle length increased to 243 (upper right), 374 (lower left), and 541 ms (lower right). Activation times are given in milliseconds and isochrones are drawn at $10 \mathrm{~ms}$ intervals. Arrows indicate the direction of propagation. 


\section{Effects of RP62719 on VT}
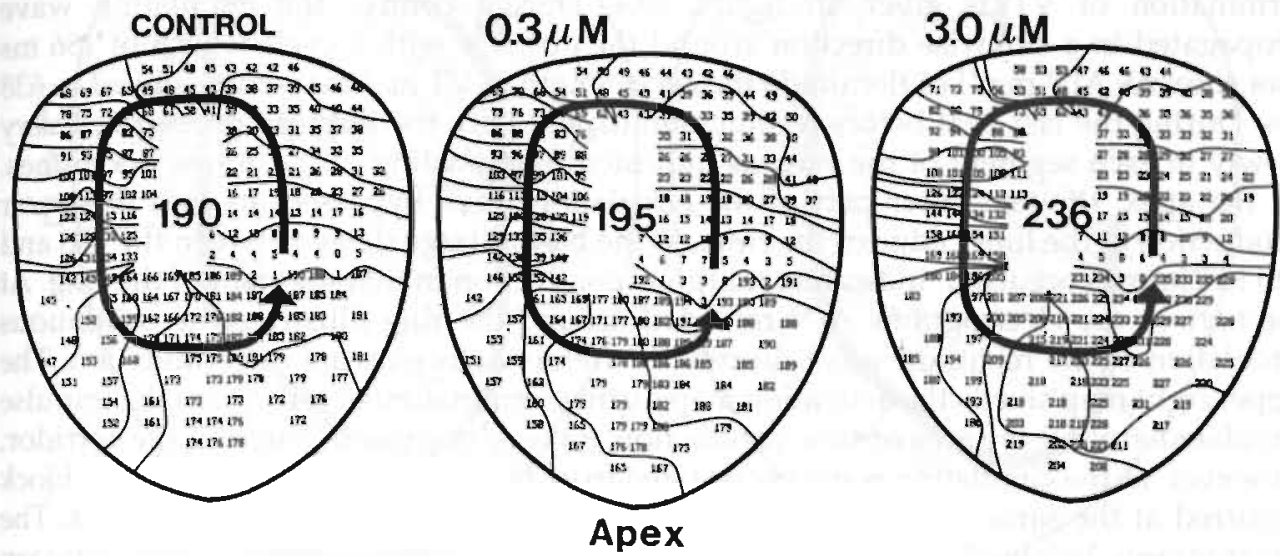

Figure 10.2: Three maps showing the activation pattern of VT during control (left), 0.3 (middle), and 3.0 $\mu M$ (right) of RP62719. During control the counterclockwise circulating wave propagated around the obstacle with a cycle length of $190 \mathrm{~ms}$. At 0.3 and $3.0 \mu \mathrm{M}$ of RP62719 the cycle length of VT increased to 195 and $236 \mathrm{~ms}$ respectively. Activation times are given in milliseconds and isochrones are drawn at $10 \mathrm{~ms}$ intervals. Arrows indicate the direction of propagation.

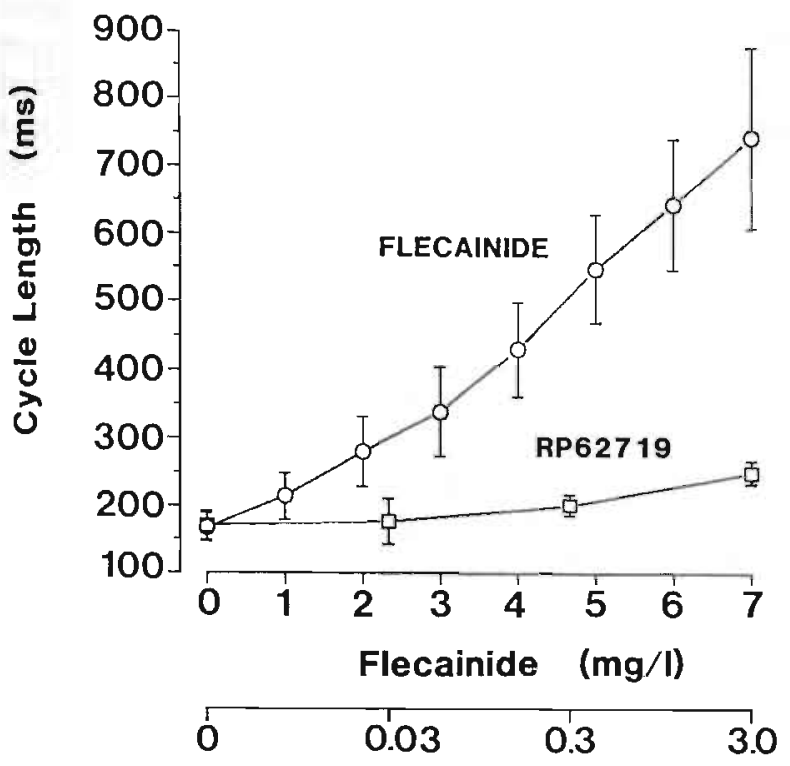

Figure 10.3: Dose-response curves of the effects of Flecainide (dots) and RP62719 (squares) on reentrant VT. The concentration of Flecainide is plotted on the upper abscissa (mg/l) while the concentration of RP62719 is plotted ( $\mu M$ ) on a logaritmic scale on the lower abscissa. The cycle length of VT is plotted on the ordinate (ms). See text for discussion.

$\operatorname{RP62719}(\mu \mathrm{M})$ 


\section{Ternnination of VT by Flecainide}

In 5 of 5 hearts, Flecainide at a concentration of $7 \mathrm{mg} / 1$ terminated VT. An example of termination of VT is given in figure 10.4. During control the circulating wave propagated in a clokwise direction around the obstacle with a cycle length of $186 \mathrm{~ms}$ (not shown). At $7 \mathrm{mg} / 1$ of flecainide the cycle length of VT had markedly slowed to 638 ms. During the last beat before termination (upper left), the impulse propagated very slowly in each segment of the ring, as indicated by crowding of the 20-ms isochrones. At the apex, the fast outer part of the circulating wave bypassed an area of slower conduction at the inner edge of the ring. At the base, a large delay between the 480 and 520 isochrones occurred, indicating marginal conduction in that segment of the ring. At the bottom the electrograms A-N recorded around the ring illustrate the continuous circulation of the reentrant wave despite the tremendous slowing of conduc-tion. The upper right map shows the activation map during termination of VT. Again the impulse circulated around the area of slow conduction at the apex prior to entering the corridor. However, as the circulating wave propagated from the base towards the free wall, block occurred at the same area where conduction was slow during the previous beat. The electrograms below illustrate the occurrence of complete conduction block between electrodes $\mathrm{L}$ and $\mathrm{M}$. In 4 of 5 hearts, the strong depression of conduction by Flecainide finally resulted in complete conduction block of the circulating wave and termination of VT. The site of conduction block was located in an area where during control conduction was fast parallel to the fiber orientation.In 1 heart the mechanism of termination of VT by Flecainide was different. Figure 10.5 shows the activation maps and electrograms during termination of VT in this heart. During control the cycle length of the clockwise VT was $157 \mathrm{~ms}$ (not shown) while $7 \mathrm{mg} / 1$ of Flecainide had increased the cycle length of VT to $756 \mathrm{~ms}$. The last beat before termination (left map) the circulating wave propagatedvery slowly in every segment of the ring, as indicated by the large number of 20 -ms isochrones. As the impulse arrived at the base of the heart the circulating impulse was partially blocked at the $600 \mathrm{~ms}$ isochrone line. A small part of the ongoing circulating wave turned around and reentered the line of functional block, thereby initiating an antidromic echo-wave. The ongoing circulating wave and the echo-wave proceeded in opposite direction and collided with each other at $t=290$ $\mathrm{ms}$ in the corridor between the LAD an the obstacle, thus terminating VT (right map).

\section{Termination of VT by RP62719}

Despite the minimal effect of RP62719 on the cycle length of VT, in 4 of 8 hearts VT was terminated at a concentration of $0.03 \mu \mathrm{M}$. In two hearts termination of VT was due to complete conduction block of the circulating wave (not shown) similar to the example of termination of VT by flecainide in figure 10.4. In the 2 other hearts VT was terminated by a reflected echo-wave after partial block of the circulating wave. An example of echo-wave termination during $0.03 \mu \mathrm{M}$ of RP62719 is given in figure 10.6. During control (upper left panel) the circulating wave propagated in a clockwise direction around the obstacle with a revolution time of $158 \mathrm{~ms}$. During RP62719 the cycle length of VT had increased to $208 \mathrm{~ms}$. The upper right panel shows the activation of the circulating wave two beats before termination of VT. At the apex two lines of functional conduction block were present between the obstacle and the outer edge of the mapping array, resulting in longitudinal dissociation of the circulating wavefront. The outer part of the wave was blocked at $t=140 \mathrm{~ms}$ but the impulse at the inner edge of the ring proceeded very slowly along the apex and the corridor to complete its 

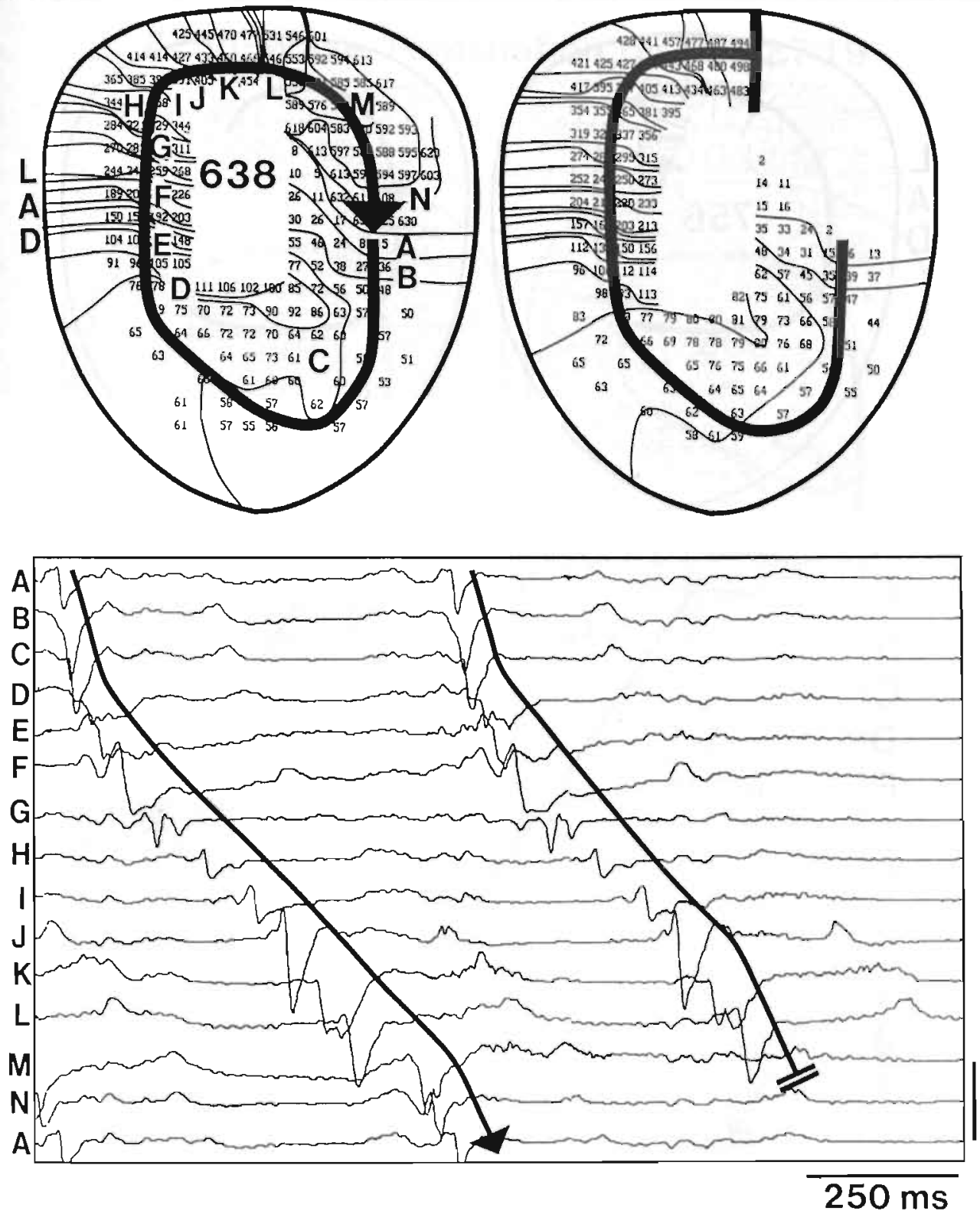

Figure 10.4: Termination of VT during $7 \mathrm{mg} / \mathrm{l}$ of flecainide. The maps at the top show the activation pattern of the last beats before termination. the last beat before termination (left map) the clockwise circulating wave proceeded very slowly around the central obstacle with a cycle length of $638 \mathrm{~ms}$. At the apex the outer part of the circulating wave bypassed an area of slow conduction at the inner edge of the ring. Complete conduction block of the impulse between sites $L$ and $M$ at the base, resulted in VT termination (right map). The 15 electrograms at the bottom were recorded at sites $A-N$ as indicated in the left activation map. Activation times are given in milliseconds. Note that the isochrones are drawn at $20 \mathrm{~ms}$ interoals. Arrows indicate the direction of propagation. Calibration bar $=5$ millivolts. A bar indicates conduction block. 

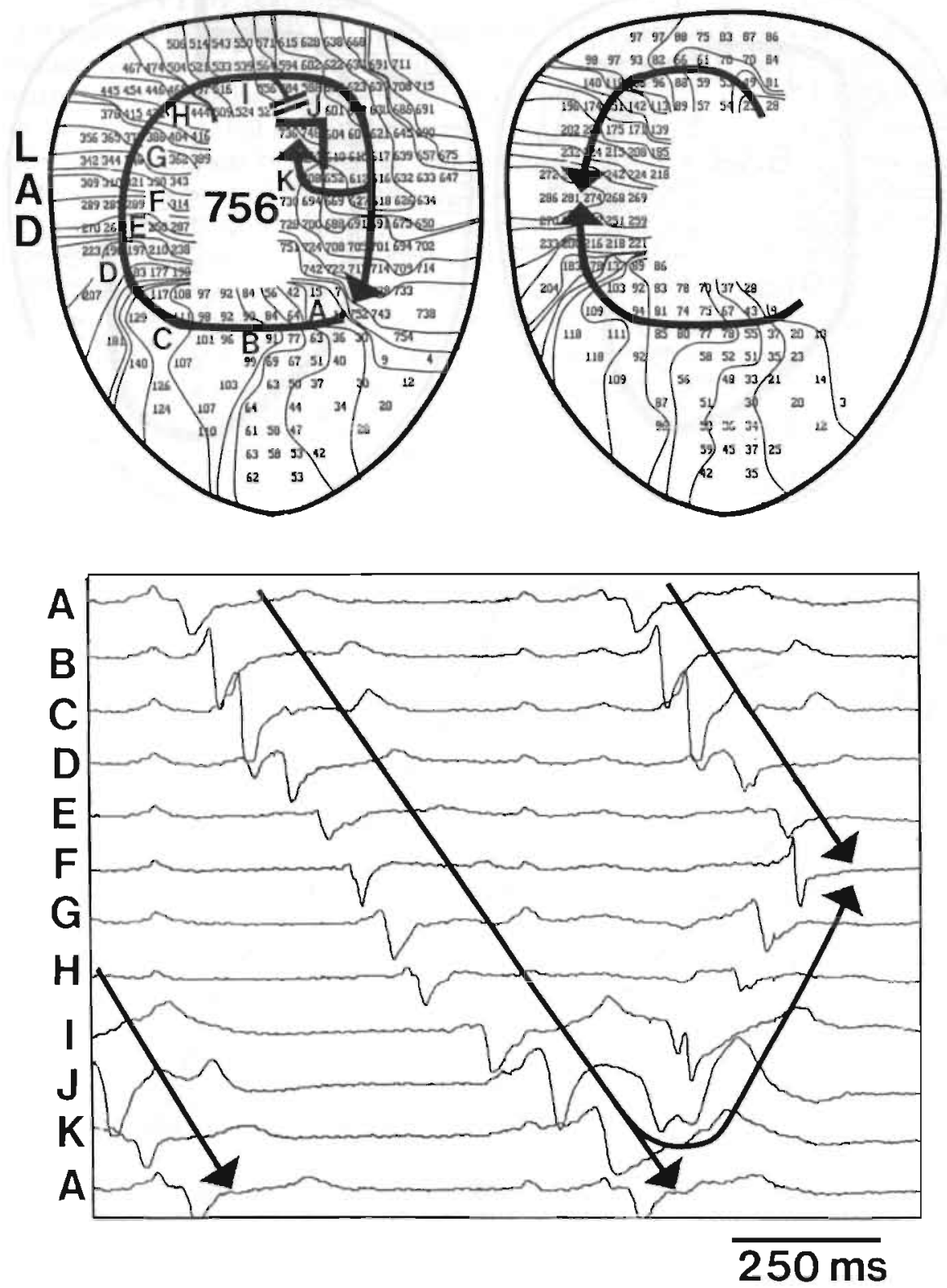

Figure 10.5: Echo-wave termination of VT by Flecainide $(7 \mathrm{mg} / \mathrm{l})$. Prior to termination the clockwise circulating wave had a cycle length of $756 \mathrm{~ms}$ (left map). At the base a part of the ongoing circulating wave reeentered a smail arc of local conduction block, initiating an antidromic echo-wave. Collision of the opposite waves at the corridor terminated VT (right map). The 12 electrograms, recorded at sites $A-K$ (left map), show that the echo-wave originating at site J collides with the ongoing circulating wave at electrode $F$. Activation times are given in milliseconds. Note that the isochrones are at $20 \mathrm{~ms}$ intervals. Arrows indicate the direction of propagation. A bar indicates conduction block. Calibration bar $=5$ milliVolts. 


\section{Echo-Wave Termination by RP62719}
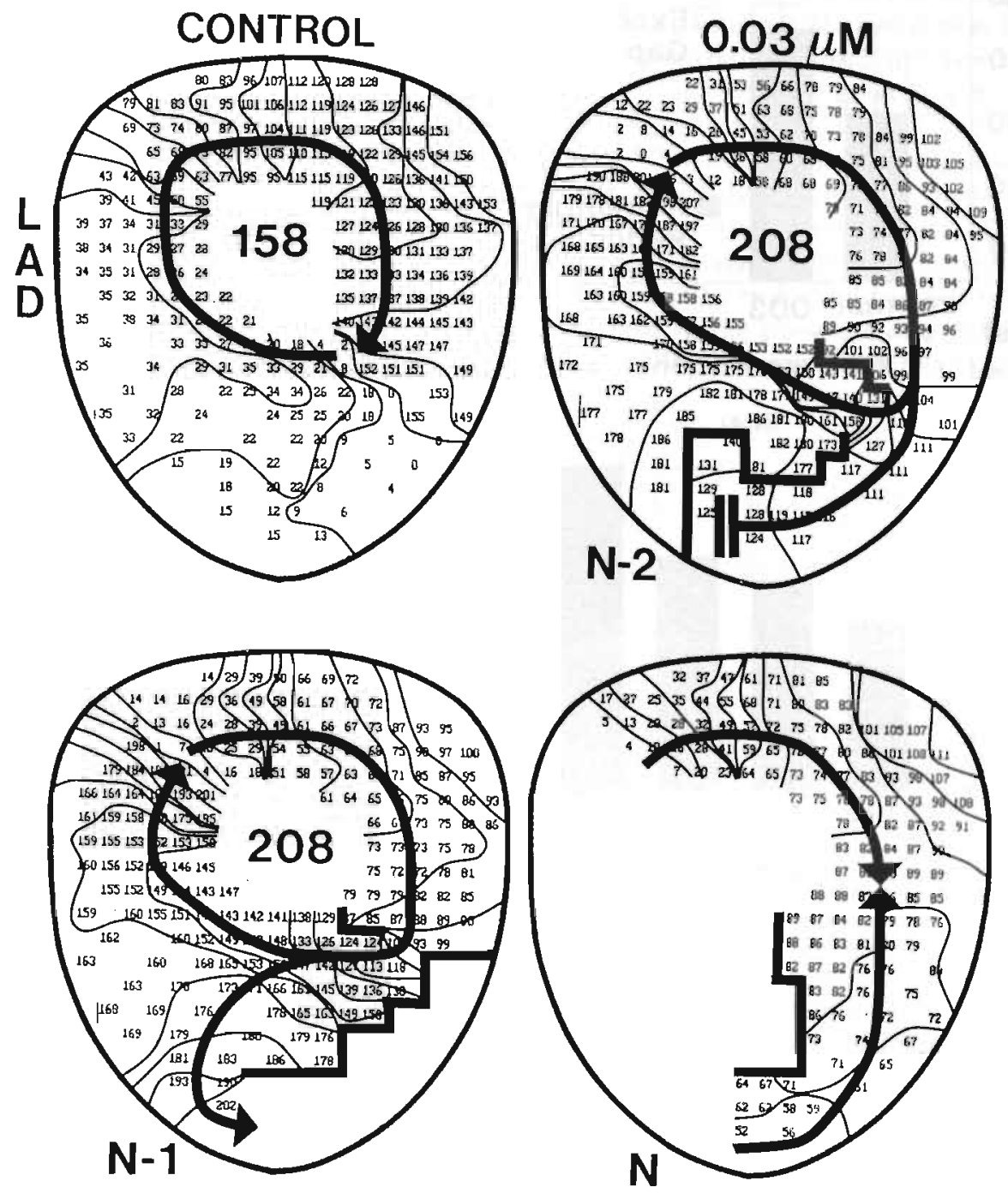

Figure 10.6: Four maps showing VT during control and echo-wave termination by RP62719 (0.05 HM). The cycle length of the clockwise VT increased from $158 \mathrm{~ms}$ during control (upper left map) to $208 \mathrm{~ms}$ during RP62719 (upper right map). Two local arcs of functional block split the circulating wave in an ongoing part at the inner edge of ring and an outer part that was blocked. During the next beat a part of the circulating wave turned around and reentered a long arc of block at the apex (lower left map). The resulting antidromic echo-wave collided with the ongoing circulating wave at the right free wall, terminating VT. Activation times are given in milliseconds and isochrone lines are drawn at $10 \mathrm{~ms}$ intervals. Arrows indicate the direction of the several waves. Bars indicate conduction block. 

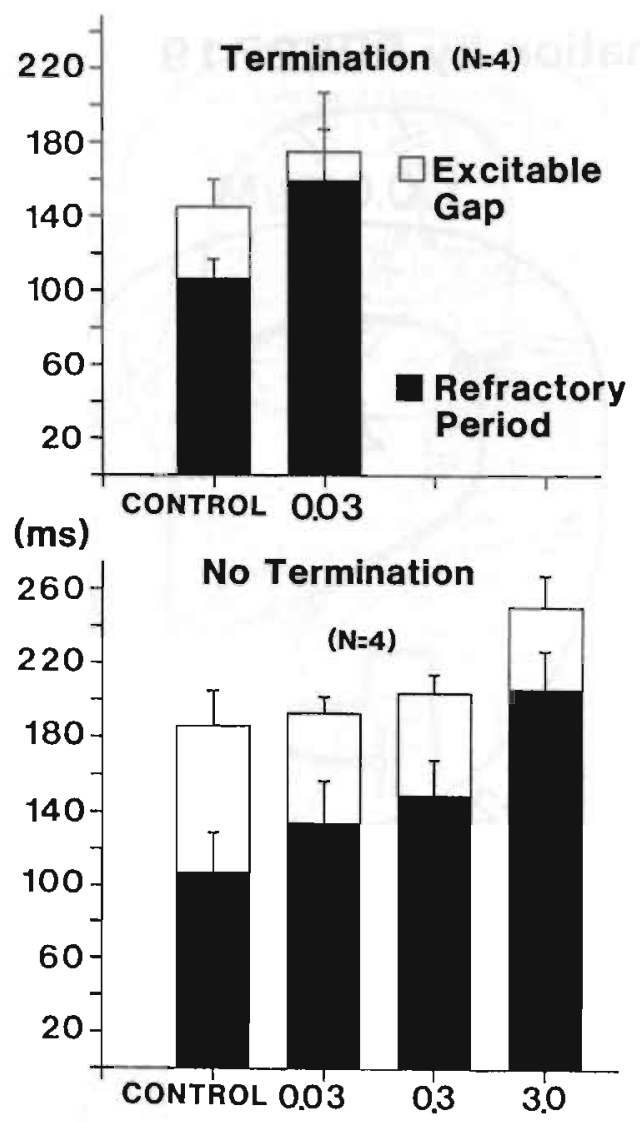

RP62719 ( $\mu \mathrm{M})$

Figure 10.7: Effects of RP62719 on the refractory period and the excitable gap of VT. The upper panel represents $4 V T$ S that could be terminated by $0.03 \mu M$ of RP62719 while 4 other VTs in the lower panel could not be terminated by the drug. The filled bars represent the refractory period while the empty bars represent the excitable gap. The sum of both bars equals the cycle length of VT. The concentration of RP62719 ( $\mu M)$ is plotted on the abscissa. The ordinate gives the average values and standard deviations of the refractory period and excitable gap. See text for discussion.

revolution. During the last beat preceding termination (lower left map) again two lines of functional conduction block were present at the outer and inner edge of the apex. As the circulating wave proceeded along the apex and the corridor, a part of the wave split off and turned around the long line of block at the outer edge of the ring. This returning wave reentered the line of block thereby initiating an antidromic echo-wave. This antidromic echo-wave collided with the clockwise circulating wave at the free wall of the left ventricle, resulting in termination of VT (lower right map).

Although in 4 of 8 hearts VT was terminated by $0.03 \mu \mathrm{M}$ of RP62719, in 4 other hearts even a 100 -fold increase in concentration to $3.0 \mu \mathrm{M}$ did not lead to termination of VT. Figure 10.7 shows the cycle length, refractory period, and excitable gap of both groups of VTs. The filled bars represent the refractory period and the empty bars the excitable gap. The filled and empty bars together represent the cycle length of VT. During control, the V'Ts that could be terminated (upper panel) were faster than the VTs that could not be terminated (lower panel) (cycle length $145 \pm 18$ versus $186 \pm 19 \mathrm{~ms}, \mathrm{p}<0.05$ ). Because the refractory periods in both groups were similar (107 \pm 20 versus $108 \pm 21 \mathrm{~ms}$ ) the excitable gap of the fast VTs was much shorter than in slow VTs (42 \pm 12 versus 79 $\pm 22 \mathrm{~ms}, \mathrm{p}<0.05)$. Conduction velocities longitudinal $\left(\theta_{\mathrm{L}}=66 \mathrm{~cm} / \mathrm{s}\right)$ and transverse $\left(\theta_{\mathrm{T}}=\right.$ 
$21 \mathrm{~cm} / \mathrm{s}$ ) to the fiber direction were the same in both groups (not shown). In the 4 VTs that were terminated by RP62719, at $0.03 \mu \mathrm{M}$ the increase in RP was more pronounced than in the 4 VTs that could not be terminated (50\% versus $26 \%$ prolongation). Although in the VTs that were terminated the cycle length of VT was also slightly prolonged, the excitable gap was shortened to such an extent that conduction block occurred at one segment of the ring conduction block occurred, resulting in termination of VT. In the other 4 VTs, the excitable gap could not be abolished even at a 100-fold higher dosis of RP62719 and therefore VT was not terminated.

In the VTs that were terminated the cycle length of VT was significantly increased at $0.03 \mu \mathrm{M}$ of RP62719. This increase in cycle length probably reflects the increase in refractory period rather than a direct effect of RP62719 on conduction velocity. Due to the shortening of the excitable gap the circulating wave is being forced to propagate in partially excitable tissue, resulting in depression of conduction. Only at $3.0 \mathrm{mM}$ of RP62719 a significant increase in cycle length occurred in the presence of a still large excitable gap. This indicates that this high dosage of RP62719 exerts a direct effect on conduction velocity.

\section{DISCUSSION}

Prevention of induction, slowing in rate, or termination of VT are considered as the main beneficial effects of anti-arrhhythmic drugs. ${ }^{.}$Slowing of conduction and termination of VT is generally assumed to result from an increase in refractoriness or a depression of conduction. ${ }^{8,4}$ In the present study, we found that in all hearts the class Ic drug Flecainide markedly slowed conduction and increased the cycle length of VT to more than $400 \%$ before termination of VT occurred at $7 \mathrm{mg} / \mathrm{l}$, a dose well above the therapeutic range. ${ }^{10,11}$ This implicates that in all segments of the ring the safety factor for conduction must have been high. In contrast, the experimental class III drug RP62719 terminated VT in only 4 of 8 hearts. Termination was preceded by a marked increase in refractory period and a marked decrease in excitable gap, suggesting that conduction block and termination of VT were due to abolition of the excitable gap at one segment of the circuit. In the VTs that were not terminated by RP62719, the refractory period and cycle length of VT were only moderately prolonged and the excitable gap was hardly decreased even at a 100 -fold higher dosage. The striking difference between both groups is that in the faster VTs RP62719 had a very pronounced effect on the refractory period, indicating a rate-dependent action of the drug.

In patients with $\mathrm{VT}$ after moycardial infarction, the reentrant circuit is very complex and incorporates an area of slow conduction. ${ }^{12-15}$ Some studies have suggested that antiarrhythmic drugs may especially affect the segment of slow conduction, providing a preferential site for conduction block and termination of VT. ${ }^{16.17}$ Previously we have demonstrated that electrical uncoupling may terminate VT by preferential conduction block in the segment of slow transverse conduction, ${ }^{18}$ while depression of the fast sodium inward current by high extracellular $\mathrm{K}^{+}$and Tetrodotoxin preferentially affected longitudinal conduction. In the present study, the sodium channel-blocker Flecainide also produced conduction block at sites showing fast longitudinal conduction during control. This is in agreement with the hypothesis postulated by Spach et al. ${ }^{1920}$ that longitudinal conduction has a lower safety factor for conduction. In addition, Spach et 
$\mathrm{al}^{21}$ demonstrated that during longitudinal conduction the binding of sodium channel blockers with the fast sodium channels was much stronger than during transverse conduction. During RP62719 no preferential area for conduction block could be detected and termination of VT did not seem to be related to tissue anisotropy.

\section{Mechanisms underlying Termination of VT}

We found that termination of VT was predominantly due to complete conduction block of the circulating wave. However, both during Flecainide (1/5 hearts) and RP62719 (2/4 hearts) a partial line of block developed, leading to micro-reentry and reflection of the circulating wave in the ring. The resulting echo-wave propagated in antidromic direction around the ring and collided with the ongoing circulating wave, thus terminating VT. Collision of the circulating wave with an antidromic impulse has also been described by Spinelli and Hoffman ${ }^{22}$ during termination of atrial flutter by dSotalol. The inducibility of a micro-reentrant echo-wave depends on the wavelength of the impulse, being the product of conduction velocity and refractory period. ${ }^{23}$ Flecainide mainly slowed conduction and thus decreased the wavelength, favouring the occurrence of micro-reentry. On the other hand, RP62719 mainly increased the refractory period which would prolong the wavelength and hamper the inducibility of echo-waves. However, as shown by our results the arcs of block during RP62719 were very long, permitting micro-reentry despite the increased wavelength.

Previous experimental studies have demonstrated that block of the circulating wave during reentry can be associated with oscillations in cycle length and local refractory period. ${ }^{24,25}$ During treatment of clinical VT with various antiarrhythmic drugs, Callans et al. ${ }^{7}$ also observed oscillations in interval. In $7 \%$ of cases these oscillations were enhanced prior to VT termination, associated with a change in QRS-morphology. In our study, in most cases termination of VT was not preceded by oscillations in interval. However, it can be expected that in case of an echo-wave termination the morphology of the last QRS-complex will be different. Further clinical studies are needed to validate the concept that echo-waves can be a mechanism for termination of VT in patients.

\section{CONDENSED ABSTRACT}

Termination of reentrant ventricular tachycardia in Langendorff perfused rings of rabbit epicardium was studied by high resolution mapping during pharmacological interventions with a class Ic (Flecainide) and experimental class III (RP62719) drug. Flecainide markedly slowed conduction and increased the cycle length of VT, while RP62719 had a much less pronounced effect on conduction velocity and the VT cycle length despite a significant prolongation of the refractory period. At very high concentrations of Flecainide VT was terminated in all hearts, whereas during RP62719 only half of the VTs was terminated by abolishing the excitable gap during VT. Independant of the pharmacological action, two mechanisms of termination of VT were identified: 1) complete conduction block of the circulating wave in a segment of the ring and 2) collision of the circulating wave with a reflected antidromic echo-wave. 


\section{REFERENCES}

1. Marchlinski F. Ventricular tachycardia: clinical presentation, course, therapy. In Zipes D, Jalife J (eds): Cardiac electrophysiology: from cell to bedside. WB Saunders Company, 1990, pp 756-777

2. Vaughan Williams E. A classification of antiarrhythmic actions reassessed after a decade of new drugs. J Clin Pharmacol 1984;24:129-147

3. Task Force of the working group on cardiac arrhythmias of the European Society of Cardiology. The Sicilian Gambit: A new approach to the classification of antiarrhythmic drugs based on their actions on arrhythmogenic mechanisms. Eur Heart J 1991;12:1112-1131

4. Wellens HJJ, Schuilenburg R, Durrer D: Electrical stimulation of the heart in patients with ventricular tachycardia. Circulation 1972;46:216-226

5. Wellens HJJ: Value and limitations of programmed electrical stimulation of the heart in the study and treatment of tachycardias. Circulation 1978;57:845-853

6. Josephson M, Horowitz L, Farshidi, Kastor J. Recurrent sustained ventricular tachycardia: 1. Mechanisms. Circulation 1978;57:431-439

7. Callans D, Marchlinsli F. Characterization of spontaneous termination of sustained ventricular tachycardia associated with coronary artery disease. Am J Cardiol 1991;67:50-54

8. Stamato N, Frame L, Rosenthal M, Almendral J, Gottlieb C, Josephson M: Procainamide-induced slowing of ventricular tachycardia with insights from analysis of resetting response patterns. Am J Cardiol 1989;63:1455-1461

9. Callans D, Hook B, Josephson M. The mechanism of propafenone-induced slowing of ventricular tachycarda in man as analyzed by the resetting response patterns. PACE 1991;14:2035-2041

10. Anderson J, Lutz J, Allison S. Electrophysiologic and antiarrhythmic effects of oral flecainide in patients with inducible ventricular tachycardia. J Am Coll Cardiol 1983;2:105-114

11. Kidwell G, Greenspon A, Greenberg R, Volosin K. Use-dependent prolongation of ventricular tachycardia cycle length by type I antiarrhythmic drugs in humans. Circulation 1993;87:118-125

12. El-Sherif N, Scherlag B, Lazzara R, Hope R. Reentrant ventricular arrhythmias in the late myocardial infarction period. 1. Conduction characteristics in the infarction zone. Circulation 1977:55:783-791

13. Wit A, Allessie M, Bonke F, Lammers W, Smeets J, Fenoglio J: Electrophysiologic mapping to determine the mechanism of experimental ventricular tachycardia initiated by premature impulses. Am J Cardiol 1982;49:166-185

14. Dillon S, Allessie M, Ursell P, Wit A. Influences of anisotropic tissue structure on reentrant circuits in the epicardial borderzone. Circ Res 1988;63:182-206

15. de Bakker J, van Capelle F, Janse M, Wilde A, Coronel R, Becker A, Dingemans K, van Hemel N, Hauer R. Reentry as a cause of ventricular tachycardia in patients with chronic ischemic heart disease. Electrophysiologic and anatomic correlation. Circulation 1988;77:589-606

16. Kadish A, Spear J, Levine J, Moore N. The effects of procainamide on conduction in anisotropic canine ventricular myocardium. Circulation 1986;74:616-625

17. Kay G, Epstein A, Plumb V. Preferential effect of procainamide on the reentrant circuit of ventricular tachycardia. J Am Coll Cardiol 1989;14:383-390

18. Brugada J, Mont Ll, Boersma L, Kirchhof C, Allessie M. Differential effects of heptanol, potassium, and tetrodotoxin on reentrant ventricular tachycardia around a fixed obstacle in anisotropic myocardium. Circulation 1991;84:1307-1318

19. Spach M, Miller W, Geselowitz D, Barr R, Kootsey M, Johson E. The discontinuous nature of propagation in normal canine cardiac muscle. Evidence for recurrent discontinuities of intracellular resistance that affect the membrane currents. Cir Res 1981;48:39-54

20. Spach M, Miller W, Dolber P, et al. The functional role of structural complexities in the propagating depolarization in the atrium of the dog. Cardiac conduction disturbances due to discontinuities of effective axial resistivity. Circ Res 1982:50:175-191

21. Spach M, Dolber P, Heidlage J, et al. Propagating depolarization in anisotropic human and canine cardiac muscle: apparent directional differences in membrane capacitance. A simplified model for selective directional effects of modifying the sodium conductance on Vmax, Tfoot, and the propagation safety factor. Circ Res 1987;60:206-219

22. Spinelli W, Hoffman B. Mechanisms of termination of reentrant atrial arrhythmias by class $I$ and class III agents. Circ Res 1989;65:1565-1579 
23. Rensma P, Allessie M, Lammers W, Bonke F, Schalij M. Length of excitation wave and susceptibility to reentrant atrial arrhythmias in normal conscious dogs. Circ Res 1988;62:395-410

24. Frame L, Simson M. Oscillations of conduction, action potential duration, and refractoriness. A mechanism for spontaneous termination of reentrant tachycardias. Circulation 1988;78:1277-1287

25. Frame L, Rhee E. Spontaneous termination of reentry after one cycle or short non-sustained runs. Role of local oscillations and excess dispersion of refractoriness. Circ Res 1991;68:493-502 


\section{CHAPTER 11}

\section{GENERAL DISCUSSION}

11.1 Anisotropic reentry in a uniform epicardial border zone.

11.2 Reentrant excitation around a fixed anatomical obstacle. 


\subsection{Anisotropic Reentry in a Uniform Epicardial Border Zone}

Ventricular arrhythmias after myocardial infarction in patients are most likely due to reentrant excitation (Wellens et al. 1972, 1978, Josephson et al. 1978, 1978). In experimental canine myocardial infarctions, ventricular arrhythmias originate from a surviving border zone of the infarct (El-Sherif et al. 1976, Karagueuzian et al. 1979, Michelson et al. 1980), and are due to functional reentry, either as figure-of-eight reentrant circuit or as a single circulating wave (El-Sherif et al. 1981, Wit et al. 1982, Mehra et al. 1983, Garan et al. 1987). In the surviving myocardium the action potential characteristics usually return to normal within two weeks after the ischemic event (Ursell et al. 1985) but scar formation and fibrosis transform the uniform anisotropic border zone into a region exhibiting abnormal, slow conduction (Karagueuzian et al. 1979, Fenoglio et al. 1983, Richards et al. 1984). Dillon et al. (1988) and Cardinal et al. (1988) have shown that this enhanced tissue anisotropy has a great influence on the characteristics of functional reentry. They showed that reentry was due to a circulating wave around a central line of functional conduction block or pseudoblock, oriented parallel to the general fiber direction.

In this thesis we have shown that also in a thin "border zone" of subepicardium with uniform anisotropic conduction properties, functional reentrant circuits can be induced with characteristics similar to that of anisotropic circuits in non-uniform myocardium. The central line of functional conduction block is oriented parallel to the epicardial fiber direction and the circuits have an ellipsoid shape. The majority of our functional VTs were based on a single circulating wave as opposed to figure-of-eight reentrant circuits found most often in other experimental studies (El-Sherif et al. 1981, Mehra et al. 1983, Dillon et al. 1988). In the frozen rabbit heart, one of the ends of a long line of functional block will usually be continuous with the artificial boundary of the left ventricle, allowing an impulse to propagate only around one end of the line of block to initiate functional VI based on a single circulating wave. In the infarcted ventricle there may not be such boundaries, allowing the impulse to propagate around both sides of a long line of conduction block and reenter the center of the line of block to initiate figure-ofeight reentry. Thus, the two forms of functional reentry probably reflect a different initiation sequence rather than a difference in nature.

We investigated the characteristics of functional reentry in anisotropic myocardium by studying the center of the reentrant circuit with micro-electrodes, and by determi-ning the response of VT to high extracellular $\mathrm{K}^{+}$concentrations and to single prema-ture stimuli. The action potentials recorded with micro-electrodes showed that during anisotropic VT an excitable gap is present at all sites around the central line of functional block. The tissue at the central line of block is kept continuously depolarized and cannot be activated by the circulating wave. At the pivoting points of the line of block the cells exhibited foot-potentials and annihilation of phase 4 of the action potential. From these observations we concluded that a low amount of excitatory current during a sharp U-turn and a large current load of the longitudinal limbs of an anisotropic circuit, result in a delay of the circulating wave at the pivoting points causing an excitable gap in the rest of the circuit. These data are in line with computer simulations by Pertsov et al. (1993) on the nature of the excitable gap during spiral wave activity. On the other hand, a raise in extracellular $\mathrm{K}^{+}$concentration preferentially affected the tissue at the central line of block by rende-ring this area inexcitable. Although during slow pacing in the epicardial sheet no gross conduction disturbances 
were visible, there may have been a lower safety factor for conduction in the region where the reentrant circuit was anchored during functional VT. Schalij et al. (1992) have shown that the cryoprocedure did not markedly change the refractory periods of the left ventricle nor did it increase the dispersion in refractoriness. However, local differences in refractoriness (Gough et al. 1985, Butrous et al. 1992) or anatomical discontinuities in conduction on a much smaller scale may still affect the course of the functional VTs in our model. Such small anatomical landmarks may contribute to the existence of an excitable gap and may provide anchoring points for a functional reentrant circuit.

Measurement of the excitable gap by application of premature stimuli during functional VT revealed that it spanned about $37 \%$ of the cycle length of VT. Despite the presence of an excitable gap, slowing of conduction of premature wavefronts within the reentrant circuit prevented reset of VT. This indicates that the excitable gap during functional reentry in uniform anisotropic myocardium consists of partially excitable tissue. Clinical studies show that VT can usually be reset with a flat reset curve, suggesting the presence of fully excitable gap and arguing against functional reentrant circuits (Almendral et al. 1986, Stamato et al. 1988). It should be noted however that clinical studies do not include fast hemodynamically less tolerated VTs, which may very well be based on functional reentry.

The effects of anti-arrhythmic drugs on functional anisotropic reentry have not yet been extensively tested in our laboratory. However, some conclusions may be drawn from the effects of high extracellular $\mathrm{K}^{+}$on functional VT $(n=2)$ in our study, the effects of flecainide on functional VT $(n=2)$ found by Brugada et al. (1991), and the effects of heptanol on VT in the study of Nassif et al. (1993). Depression of the active membrane: properties by raising the extracellular concentration of $\mathrm{K}^{+}$from 4 to $10 \mathrm{mM}$ slowed functional VT by $60 \%$ without terminating the arrhythmia. Brugada et al. (1991) showed that administration of a therapeutic dose (Anderson et al. 1983) of $1 \mathrm{mg} / 1$ of Flecainide prolonged the VT interval by $66 \%$ compared to control. Nassif et al. (1993) found a similar prolongation in cycle length during electrical uncoupling of the cells with 1.2 $\mathrm{mM}$ of heptanol. From these observations it can be concluded that high $\mathrm{K}^{+}$, Flecainide, and Heptanol all result in slowing VT by depression of conduction, although at nontoxic concentrations neither of these agents has the capacity to terminate functional VT in uniform anisotropic myocardium. It has been shown that in healed infarcted myocardium, areas of slow conduction are much more sensitive to uncoupling of the cells by low doses of heptanol (Spear et al. 1990). If the same holds true for agents that depress the active membrane properties, functional VTs in non-uniform anisotropic tissue may be more easily abolished by anti-arrhythmic agents. Future experiments should be directed to find out which type of anti-arrhythmic agent is most suitable to depress conduction in non-uniform anisotropic circuits and to abolish VT. Such studies may be performed in experi-mental models with an enhanced degree of anisotropy, either by creating a myocar-dial infarction or by a selective depression of conduction in different regions of a reentrant circuit. In view of the large amount of evidence from experimental models of myocardial infarction (El-Sherif et al. 1981, Wit et al. 1982, Mehra et al. 1983, Richards et al. 1984, Garan 1987, Cardinal et al. 1988, Dillon et al. 1988), we assume that anisotropic reentry provides a mechanism for at least a subset of VTs in patients (Gallagher 1985, Downar 1988). 


\subsection{Reentrant Excitation around a Fixed Anatomical Obstacle}

Histologic and electrophysiologic studies of human hearts after myocardial infarc-tion have revealed that thin layers of endocardium or epicardium can survive the ischemic event (Josephson et al. 1978, Horowitz et al. 1980, Fenoglio et al. 1983, Littman et al. 1991). In man, most arrhythmias that are induced after infarction seem to have a focal origin at small areas of a few square centimeters and are only rarely based on a macroreentry pathway around an infarction scar (Josephson et al. 1978, de Bakker et al. 1988). This focal origin may be a reflection of micro-reentry in a very small circuit but it may also be the exit point of a large intramural circuitous pathway. Studies by de Bakker et al. $(1988,1990)$ have provided evidence that in infarcted human hearts, VT could be based on macro-reentrant circuits, consisting of surviving muscle bundles within the infarcted tissue connecting different regions of subendocardial tissue. In ventricular tachycardia after myocardial infarction, subsequent QRS-complexes in the surface ECG are separated by iso-electric diastolic intervals. If reentry is the mechanism for VT the long diastolic intervals must be explained by a segment of very slow conduction within the reentrant circuit that does not contribute to the QRS-complex. The surviving tracts of myocardium described by de Bakker et al. $(1988,1990)$ may provide such segments of slow conduction. In these tracts the action potential characteristics are close to normal (Fenoglio et al. 1983, de Bakker et al. 1988) and slow impulse propagation is due to enhanced tissue anisotropy (de Bakker et al. 1990). Studies on experimentally infarcted canine myocardium have shown similar non-uniform anisotropic conduction properties in the epicardial border zone (Ursell et al. 1983, Richards et al. 1984, Garan et al. 1984).

In our laboratory we have created a model of 2-D rings of perfused anisotropic ventricular myocardium in which sustained monomorphic reentrant VT can be easily induced. In these rings a segment of slow conduction $(<30 \mathrm{~cm} / \mathrm{s})$ exists between the LAD and the central obstacle while in the other segments of the circuit conduction is faster. The differences in conduction velocity around the circuit are due to the normal anisotropic tissue structure of the ventricular myocardium. In the corridor between the LAD and the central obstacle the impulse propagates slowly transverse to the local fiber orientation while at the base and free wall fast impulse propagation occurs parallel to the fibers. In these anisotropic rings of myocardium, reentrant VT was easily and reproducibly induced by application of multiple extrastimuli or by incremental pacing. The anisotropic tissue structure had a great influence on the mechanism of initiation of VT. We found that during initiation of VT by multiple extrastimuli the site of unidirectional block was always located in the segment of fast longitudinal conduction at the base and free wall of the left ventricle. This is in line with the postulations of Spach et al. $(1981,1982)$ that conduction parallel to the fiber orientation is fast but has a lower safety factor for conduction and is therefore more susceptible to conduction block than transverse conduction. During incremen-tal pacing, conduction block preferentially occurred in the corridor between the LAD and the central obstacle, indicating a preferential depression of transverse impulse propagation at high rates. Other investigators made similar observations (Spach et al. 1982, Tsuboi et al. 1985) which were attributed to an increased coupling resis-tance by intracellular $\mathrm{Ca}^{++}$accumulation (Loewenstein et al. 1966). Previously, it has been shown that a large spatial dispersion in refractoriness may also provide a basis for uni-directional block and reentry of the cardiac impulse (Han and Moe 1964, Allessie et al. 1976, Gough et al. 1983). In our 
model both during regular pacing and during VT the refractory periods were not significantly different in different seg-ments of the circuit. Although small local differences in refractoriness existed, they could contribute to less than half of the cases of uni-directional block. In 2-D rings of myocardium the uniform anisotropic tissue structure by itself provides enough inhomogeneity in conduction for uni-directional conduction block and initiation of reentrant excitation.

\section{Programmed Electrical Stimulation during Anatomic VT}

We determined the characteristics of anatomic VT by application of single premature stimuli and trains of stimuli. Single premature stimuli revealed the existence of a large excitable gap (43\% of the VT interval) at every site of the circuit. During progressively premature stimuli the largest part of the reset curve was completely flat, indicating that the excitable gap consisted of fully excitasble tissue. Even the earliest premature stimulus did not result in termination of VT, due to a high safety factor for conduction at every site in the circuit. In clinical VTs, the late part of the reset curve is usually flat which is taken as evidence for macro-reentry with a fully excitable gap (Almendral et al. 1986a). However, at increasing prematurity the early part of the curve is often increasing and up to $40 \%$ of VTs can be terminated by premature stimuli (Almendral et al. 1986b, Stamato 1987). This indicates that the reentrant circuit in patients exhibits decremental conduction properties as opposed to the normal anisotropic conduction during reset of our circuits.

The effects of rapid trains of stimuli on VT were tested at several pacing sites around the circuit (chapter 8). The activations patterns that were observed during rapid pacing corrseponded to the indirect clinical criteria for entrainment proposed by Waldo et al. $(1983,1989)$ and Henthorn et al. (1988). During entrainment at long intervals, a paced antidromic wavefront would enter the circuit to extinguish the circulating wave while a paced orthodromic wave restarted VT. Okumura et al. $(1985,1987)$ postulated that the criteria for entrainment can only be fullfilled during pacing proximal but not distal to the segment of slow conduction. Our results demonstrate that only during pacing proximal to the segment of slow conduction a large part of the ventricle will be activated from a different direction, which would lead to fusion beats in a surface ECG. At very short coupling intervals entrainment resulted in termination, changes in morphology or acceleration of VT. Termination was most often due to complete conduction block of the paced orthodromic circula-ting wave, as has been proposed (Okumura et al. 1987, Aizawa et al. 1992) or observed in other studies (Waldecker et al. 1993). In the other cases, partial block of the paced orthodromic wave allowed local micro-reentry and reflection of an antidromic echo-wave in the ring which collided with the ongoing circulating wave, resulting in termination of VT. Conduction block at short pacing intervals preferen-tially occurred in the segment of slow transverse conduction, similar to findings of Spach et al. (1982) and Tsuboi et al. (1985). Conduction block was preceded by oscillations in interval at the site of block, which has also been observed by Frame et al. $(1988,1990)$ during termination of atrial reentry. Conduction block during entrainment of clinical VTs has been attributed to decremental conduction properties of the reentrant circuit (Okumura et al. 1987, Aizawa et al. 1992). However, Callans et al. (1993) have shown that depression of conduction during entrainment may be due to reset of the reset circuit, and that the conduction properties of the reentrant circuit may only be reliably determined by application of single premature stimuli. In our studies, we found several possible mechanisms for the pro-arrhythmic effects of 
entrainment which are observed in $40 \%$ of clinical VTs (Waldecker et al. 1986). A change in morphology of VT occurred when after entrainment the direction of propagation in the circuit had reversed. Acceleration of VT after entrainment was due to two wavefronts propagating simultaneously in the same circuit and in the same direction, a phenomenon which we have called double-wave reentry (Brugada et al. 1990). Double-wave reentry occurred only in slow VTs with a high ratio between the excitable gap and the refractory period. In another study from our laboratory (Brugada et al. 1991) additional mechanisms of acceleration were obser-ved, including a change from an anatomical to a functional circuit or a change between two different anatomical circuits. El-sherif et al. (1987) and Dillon et al. (1993) observed that acceleration could also result from a change of the central line of block of functionally determined circuits. So far, direct clinical evidence for either of these mechanisms is scarce (Fitzgerald et al. 1991) and awaits further study.

\section{Anti-Arrhythmic Agents during Anatomic VT}

Anti-arrhythmic drugs are an important tool in the management of ventricular tachycardia in patients. The beneficial goals of drug treatment, including prevention of initiation and slowing and termination of VT, are reached in about 30-40\% of patients (Marchlinski 1990). The most commonly used agents in this respect either mainly slow impulse propagation (Class Ic) or mainly prolong refractoriness (Class III), or exert a combined effect (Class Ia). The magnitude of the effects of most of these anti-arrhythmic drugs on clinical VT are well known (Kidwell et al. 1993, Markel et al. 1993), but the mechanisms by which slowing and termination of VT occurs are unclear. Stamato et al. (1989), Callans et al. (1991)and Hook et al. (1989) utilized the reset response to indirectly show that both class I (procainamide and propafenone) and class III (amiodarone) drugs increased the cycle length of clinical VT mainly by a direct slowing of conduction in the reentrant circuit. Callans et al. (1991) showed that termination of clinical VTs could be preceded by variations in interval, without providing evidence for the underlying mechanism.

In our experiments (chapters 9 and 10), we tested the effects of several different agents on reentry around an anatomic obstacle. Heptanol, high extracellular $\mathrm{K}^{+}$, and the class Ic agents Flecainide and Org7797 mainly slowed impulse propagation with only a small effect on refractoriness, resulting in an increase in cycle length of VT. Heptanol slows conduction mainly by increasing the intercellular resistance by block of the gap junctions increasing the intercellular resistance (Oxford et al. 1979, Balke et al. 1988, Jalife et al. 1989), while high $\mathrm{K}^{+}$, Flecainide, and Org7797 slow conduction mainly by reducing the fast sodium inward current. The present and previous studies from our laboratory (Brugada et al. 1991) show that agents that slowed impulse propagation could terminate anatomic VT. However, termination only occurred after toxic concentrations of each substance had seriously depressed impulse propagation and the VT interval had increased to about $380 \%$ compared to control, again indicating the high safety factor for conduction in our uniform anisotropic circuits.

The class III agents d-Sotalol and RP62719 manly increased the refractory period during VT. The increase in refractoriness by toxic concentrations of d-Sotalol was only moderate due to its reverse rate-dependent action (Hafner et al. 1988, Funck-Brentano et al. 1991), while RP62719 markedly increased refractoriness during fast but not during slow VTs. This may reflect a rate-dependent action of the drug although there have been no previous reoprts of such an effect. Slowing of conduc-tion by both class III 
drugs was minimal and only occurred at toxic concentrations. RP62719 only terminated fast VTs while d-Sotalol never terminated VT. The VTs that could be terminated by RP62719 had in common a short excitable gap which at some site within the ring could be abolished by increasing refractoriness leading to block of the circulating wave, as opposed to slow VTs where the excitable gap was too large to be abolished and VT was not terminated. These results are somewhat at odds with studies by Spinelli and Hoffman (1989) showing that class III agents may stop atrial reentry without abolishing the excitable gap.

We found that termination of VT by anti-arrhythmic drugs was due to two different mechanisms. The most common reason for termination was complete conduction block of the circulating wave which abolished VT. Block of the circulating wave occurred rather sudden and was not associated with oscillations in intervals as opposed to observations of Frame et al. (1988) and Callans et al. (1991). The other mechansism for termination of VT was partial block of the circulating wave, resulting in micro-reentry and initiation of an antidromic echo-wave within the circuit that collided with the ongoing circulating wave. Echo-wave termination was a common mechanism for termination of VT independent of the pharmacological agent (Brugada et al. 1992). In studies on termination of atrial reentry Spinelli and Hoffman (1989) also observed that the circulating wave could be abolished by collision with an antidromic wave, although they could not document the origin of this new impulse.

The effects of the various pharmacological agents on refractoriness and the cycle length of VT modified the response to programmed electrical stimulation. We found that drugs that mainly slowed conduction without increasing refractoriness (Org7797 and Heptanol), increased the ratio between the excitable gap and the refractory period which enhanced the susceptibility to acceleration of VT by double-wave reentry. Conversely, prolongation of the refractory period by d-Sotalol decreased the ratio between the excitable gap and the refractory period, resulting in termination and prevention of initiation of double-wave reentry.

There is abundant evidence that the reentrant circuit after moycardial infarction comprises an area of slow abnormal conduction (Okumura et al. 1985, 1987, de Bakker et al. 1988). The ideal anti-arrhythmic agent would preferentially affect that region of slow conduction at a low dosage, without affecting the non-infarcted myocardium. In the present and previous studies from our laboratory, depression of the active membrane properties by high extracellular $\mathrm{K}^{+}$, Tetrodotoxin (Brugada et al. 1991), and Flecainide, preferentially affected fast longitudinal propagation. Slow conduction due to normal tissue anisotropy was preferentially affected during blockade of the gap junctions by Heptanol (Brugada et al. 1991). Class III drugs had no differential directional effects on conduction or refractoriness. During all drugs, termination of VT only occurred after an excessive depression of conduction at toxic concentrations. Clinical reentrant circuits comprise regions of non-uniform aniso-tropic tissue structure (Fenoglio et al. 1983, de Bakker et al. 1988) which may be much more sensitive to Heptanol (Spear et al. 1990). Electrical uncoupling might then be a very effective intervention to abolish clinical VT. On the other hand, some evidence exists that class I drugs also preferentially affect the area of slow conduc-tion in clinical VTs (Kay et al. 1989). Further studies on non-uniform reentrant circuits should adress the question which type of pharmacological agent is more suitable for anti-arrhythmic therapy of reentrant VT. 


\section{Future Lines of Research}

So far, we have only tested the effects of programmed electrical stimulation and antiarrhythmic drugs only during functional and anatomical VT in normal aniso-tropic myocardium. In diseased hearts, besides tissue anisotropy other inhomogenei-ties in conduction or influences of the central nervous system may contribute to arrhythmogenesis. It may be worthwhile to mimick such altered conditions in the frozen heart model to assess their contribution to the arrhythmic propensity of the model.

One modification of the basic model may be to create a local inhomogeneity in refractoriness and/or conduction velocity by selectively changing these parameters at a given site. The first results of such an intervention have been reported by Zetelaki et al. (submitted), who showed that regional cooling selectively increased refractoriness and slowed conduction, providing a substrate for polymorphic ventricular tachycardia. The influence of the central nervous system on arrhythmogenesis may be studied by administration of an adrenergic substance either globally or selectively. Schalij et al. (1988) have shown that adrenaline is potentially arrhythmogenic because it shortens the wavelength.

The arrhythmogenic influence of secundary complications of myocardial infarction, such as congestive heart failure, may be tested by performing artificial ventricular dilatation. The first results of such an intervention have been presented by Reiter et al. (1994) who showed that acute dilatation during anatomic VT reversed the antiarrhythmic effects of d-Sotalol and enhanced the susceptibility to double-wave reentry by a shortening of refractoriness.

Another way to modify the preparation would be to create a 2-D epicardial sheet in rabbit hearts with a previous myocardial infarction. Such a preparation would mimick more closely the non-uniform substrate of VT after myocardial infarction and would allow a simple and direct study of the conduction properties of infarcted tissue.

\section{Conclusions}

A simple model of sustained monomorphic reentrant VT in 2-D rings of normal anisotropic rabbit myocardium has been developed. The uniform anisotropic struc-ture of the myocardium was of major influence both for initiation of VT and for the response of VT to programmed electrical stimulation (PES) and pharmacological interventions. Due to the high safety factor for conduction of the healthy myocar-dium, VT was not easily terminated either by programmed electrical stimulation or by drugs. In that respect, it is not a representative model for VT after myocardial infarction in patients. Nevertheless, this model has provided evidence for previously unrecognized mechanisms underlying possible pro- and anti-arrhythmic actions of programmed electrical stimulation and anti-arrhythmic drugs. Future experimental and clinical studies are required to determine the significance of our study for clinical VT after myocardial infarction. 


\section{REFERENCES}

Aizawa $Y$, Niwano S, Chinushi $M$, et al. Incidence and mechanism of interruption of reentrant ventricular tachycardia with rapid ventricular pacing. Circulation 1992;85:589-595

Allessie M, Bonke F, Schopman F. Circus movement in rabbit atrial muscle as a mechanism of tachy cardia. II. The role of non-uniform recovery of excitability in the occurrence of unidirectional block, as studies with multiple microelectrodes. Circ Res 1976;39:9-18

Almendral J, Stamato N, Rosenthal M, et al. Resetting response patterns during sustained ventricular tachycardia: relationship to the excitable gap. Circulation 1986a;74:722-730

Almendral J, Rosenthal M, Stamato N, et al. Analysis of the resetting response in sustained uniform ventricular tachycardia: incidence and relation to termination. J Am Coll Cardiol 1986b;8:294-300

Anderson J, Lutz J, Allison S. Electrophysiologic and antiarrhythmic effects of oral flecainide in patients with inducible ventricular tachycardia. J Am Coll Cardiol 1983;2:105-114

de Bakker J, Van Capelle F, Janse M, et al. Reentry as a cause of ventricular tachycardia in patients with chronic ischemic heart disease: electrophysiologic and anatomic correlation. Circulation 1988;77:589-606

de Bakker J, Coronel R, Tasseron, et al. Ventricular tachycardia in the infarcted Langendorffperfused human heart: role of the arrangement of surviving cardiac fibers. J Am Coll Cardiol 1990;15:1594-1607

Balke C, Lesh M, Spear J, et al. Effects of cellular uncoupling on conduction in anisotropic canine ventricular myocardium. Circ Res 1988;63:879-892

Brugada J, Boersma L, Kirchhof C, et al. Double-wave reentry as a mechanism of ventricular tachycardia acceleration. Circulation 1990;81:1633-1643

Brugada J, Brugada P, Boersma L, et al. On the mechanisms for ventricular tachycardia acceleration during programmed electrical stimulation. Circulation 1991;83:1621-1629

Brugada J, Boersma L, Kirchhof C, et al. Proarrhythmic effects of Flecainide. Experimental evidence for increased susceptibility to reentrant arrhythmias. Circulation 1991;84:1808-1818

Brugada J, Boersma L, Kirchhof C, et al. Echo-wave termination of ventricular tachycardia. A common mechanism of termination of reentrant arrhythmias by various pharmacological interventions. Circulation 1992;85:1879-1887

Butrous G, Gough W, Restivo M, et al. Adrenergic effects on reentrant ventricular tachycardia in subacute myocardial infarction. Circulation 1992;86:247-254

Callans D, Marchlinski F. Characterization of spontaneous termination of sustained ventricular tachycardia associated with coronary artery disease. Am J Cardiol 1991;67:50-54

Callans D, Hook B, Josephson M. The mechanism of propafenone-induced slowing of ventricular tachycardia in man as defined by analysis of resetting response patterns. PACE 1991;14:2035-2041

Callans D, Hook B, Josephson M. Comparison of resetting and entrainment of uniform sustained ventricular tachycardia. Further insights into the characteristics of the excitable gap. Circulation 1993;87:1229-1238

Cardinal R, Vermeulen M, Shenasa M, et al. Anisotropic conduction and functional dissociation of ischemic tissue during reentrant ventricular tachycardia in canine myocardial infarction. Circulation 1988;77:1162-1176

Dillon S, Allessie M, Ursell P, et al. Influences of anisotropic tissue structure on reentrant circuits in the epicardial border zone of subacute canine infarcts. Circ Res 1988;63:182-206

Dillon S, Coromilas J, Waldecker B, et al. Effects of overdrive stimulation on functional reentrant circuits causing ventricular tachycardia in the canine heart. Mechanisms for resumption or alteration of tachy-cardia. J Cardiovasc Electrophysiol 1993 (in press)

Downar E, Harris L, Mickleborough L, et al. Endocardial mapping of ventricular tachycardia in the intact human heart: evidence for reentrant mechanisms. J Am Coll Cardiol 1988;11:783-791

El-Sherif N, Scherlag B, Scherlag R, et al. Reentrant arrhythmias in the late myocardial infarction period. 1. Conduction characteristics in the infarction zone. Circulation 1976;5:686-701

El-Sherif N, Gough W, Restivo M. Reentrant ventricular arrhythmias in the late myocardial infarction period. 14. Mechanisms of resetting, entrainment, acceleration, or termination of reentrant tachycardia by programmed electrical stimulation. PACE 1987;10:341-371

Fenoglio J, Pham T, Harken A, et al. Recurrent sustained ventricular tachycardia: structure and ultrastruc-ture of subendocardial regions in which tachycardia originates. Circulation 


\section{3;68:518-533}

Fitzgerald D, Friday K, Yeung-Lai-Wah J, et al. Myocardial regions of slow conduction participating in the reentrant circuit of multiple ventricular tachycardias: Report on ten patients. J Cardiovasc Electrophysiol 1991;2:193-206

Frame L, Simson M. Oscillations of conduction, action potential duration, and refractoriness. A mechanism for spontaneous termination of reentrant tachycardias. Circulation 1988;78:1277-1287

Frame L, Rhee E. Spontaneous termination of reentry after one cycle or short non-sustained runs. Role of oscillations and excess dispersion of refractoriness. Circ Res 1991;68:493-502

Funck-Brentano C, Kibleur Y, Le Coz F, et al. Rate-dependence of sotalol-induced prolongation of ventricular repolarization during exercise in humans. Circulation 1991;83:536-545

Gallagher J, Del Rossi A, Fernandez J, et al. Cryothermal mapping of recurrent ventricular tachycardia in man. Circulation 1985;71:733-739

Garan H, Fallon J, Rosenthal S, et al. Endocardial, intramural, and epicardial activation patterns during sustained monomorphic ventricular tachycardia in late canine myocardial infarction. Circ Res 1987;60:879-896

Gough W, Mehra R, Restivo M, et al. Reentrant ventricular arrhythmias in the late myocardial infarction period. 13. Correlation of activation and refractory maps. Circ Res 1985;57:432-442

Hafner D, Berger F, Borchard U, et al. Electrophysiological characterization of the class III activity of Sotalol and its enantiomers: new interpretation of use-dependent effects. Drug Res 1988;38:231236

Han J, Moe G. Nonuniform recovery of excitability in ventricular muscle. Circ Res 1964;14:44-60

Henthorn R, Okumura K, Olshansky B, et al. A fourth criterion for transient entrainment: the electrogram equivalent of progressive fusion. Circulation 1988;77:1003-1012

Horowitz L, Josephson M, Harken A. Epicardial and endocardial activation during sustained ventricular tachycardia in man. Circulation 1980;61:1227-1238

Jalife J, Sicouri S, Delmar M, et al. Electrical uncoupling and impulse propagation in isolated sheep Purkinje fibers. Am J Physiol 1989;257:H179-H189

Josephson M, Horowitz L, Farshidi A. Continuous local electrical activity. A mechanism of recurrent ventricular tachycardia. Circulation 1978;57:659-665

Josephson M, Horowitz L, Farshidi A, et al. Recurrent sustained ventricular tachycardia: I. Mechanisms. Circulation 1978;57:431-439

Josephson M, Horowitz L, Farshidi A, et al. Recurrent sustained ventricular tachycardia: 1. Mechanisms. circulation 1978;57:431-439

Karagueuzian H, Fenoglio J, Weiss M, et al. Protracted ventricular tachycardia induced by premature stimulation of the canine heart after coronary artery occlusion and reperfusion. Circ Res 1979;44:833-846

Kay G, Epstein A, Plumb V. Preferential effect of procainamide on the reentrant circuit of ventricular tachycardia. J Am Coll Cardiol 1989;14:382-390

Kidwell G, Greenspon A, Greenberg R, et al. Use-dependent prolongation of ventricular tachycardia cycle length by type I antiarrhythmic drugs in humans. Circulation 1993;87:118-125

Littman L, Svenson R, Gallagher J, et al. Functional role of the epicardium in the post-infarction ventricular tachycardia: observations derived from computerized epicardial activation mapping, entraiment, and epicardial laser photoablation. Circulation 1991;83:1577-1591

Loewenstein W. Permeability of membrane junctions. Ann NY Acad Sci 1966;137:441-472

Marchlinski F. Ventricular tachycardia: clinical presentation, course, and therapy. In: Cardiac Electrophy-siology. From cell to bedside. Eds. Zipes D, Jalife J, WB Saunders Company, 1990:756777

Markel M, Miles W, Luck J, et al. Differential effects of isoproterenol on sustained ventricular tachycardia before and during procainamide and quinidine antiarrhythmic therapy. Circulation 1993;87:783-792

Mehra R, Zeiler R, Gough W, et al. Reentrant ventricular arrhythmias in the late myocardial infarction period. 9. Electrophysiologic anatomic correlation of reentrant circuits. Circulation 1983;67:11-24

Michelson E, Spear J, Moore E. Electrophysiologic and anatomic correlates of sustained ventricular tachyarrhythmias in a model of chronic myocardial infarction. Am J Cardiol 1980;45:583-590

Nassif G, Dillon S, Rayhill S, et al. Reentrant circuits andthe effects of heptanol in a rabbit model of 
infarction with a uniform epicardial border zone. J Cardiovasic Electrophysiol 1993;4:112-133

Okumura K, Henthorn R, Epstein A, et al. Further observations on transient entrainment: importance of pacing site and properties of the components of the reentrant circuit. Circulation. 1985;72:1293-1307

Okumura K, Olshansky B, Henthorn R, et al. Demonstration of the presence of slow conduction during sustained ventricular tachycardia in man: use of transient entrainment. Circulation 1987;75:369-378

Oxford G, Swenson R. n-Alkanols potentiate sodium channel inactivation in squid giant axons. Biophys J 1979;26:585-590

Pertsov A, Davidenko J, Salomonsz R, et al. Spiral waves of excitation underlie reentrant activity in isolated cardiac muscle. Circ Res 1993;72:631-650

Reiter M, Zetelaki Z, Boersma L, et al. Interaction of acute ventricular dilatation and d-Sutalol during sustained reentrant ventricular tachycardia around a fixed obstacle. Circulation 1994;89:(in press)

Richards D, Blake G, Spear J, et al. Electrophysiologic substrate for ventricular tachycardia: correlation of properties in vivo and in vitro. Circulation 1984;69:369-381

Schalij MJ. Anisotropic conduction and ventricular tachycardia. Doctoral Thesis, University of Limburg, Maastricht, The Netherlands, 1988

Schalij M, Lammers W, Rensma P, et al. Anisotropic conduction and reentry in perfused epicardium of rabbit ventricle. Am J Physiol 1992;263:H1466-H1478

Spach $\mathbf{M}$, Miller W, Geselowitz D, et al. The discontinuous nature of propagation in normal canine cardiac muscle. Evidence for recurrent discontinuities of intracellular resistance that affect the membrane currents. Circ Res 1981;48:35-54

Spach M, Miller W, Dolber P, et al. The functional role of structural complexities in the propagating depolarization in the atrium of the dog. Cardiac conduction disturbances due to discontinuities of effective axial resistivity. Circ Res 1982,50:175-191

Spach M, Kootsey J, Sloan J. Active modulation of electrical coupling between cardiac cells of the dog. A mechanism for transient and steady-state variations in conduction velocity. Circ Res $1982 ; 51: 347-362$

Spear J, Balke C, Lesh M, et al. Effect of cellular uncoupling by heptanol on conduction in infareted myocardium. Circ Res 1990;66:202-217

Spinelli $\mathbf{W}$, Hoffman B. Mechanisms of termination of reentrant atrial arrhythmias by class I and class III antiarrhythmic agents. Circ Res 1989;65:1565-1579

Stamato N, Rosenthal M, Almendral J, et al. The resetting response of ventricular tachycardia to single and double extrastimuli: implications for the excitable gap. Am J Cardiol 1987;60:596-601

Stamato N, Frame L, Rosenthal M, et al. Procainamide-induced slowing of ventricular tachycardia with insights from analysis of resetting response patterns. Am J Cardiol 1989;63:1455-1461

Tsuboi N, Kodama I, Toyama J, et al. Anisotropic conduction properties of canine ventricular muscle. Influences of high extracellular $\mathrm{K}+$ concentration and stimulation frequency. Jpn Circ J 1985; $49: 487-498$

Ursell P, Gardner P, Albela A, et al. Structural and electrophysiological changes in the epicardial border zone of canine myocardial infarcts during infarct healing. Circ Res 1985;56:436-451

Waldecker B, Brugada P, Zehender $\mathrm{M}$, et al. Modes of electrical termination of ventricular tachycardia: importance for the selection of implantable antitachycardia device. Am J Cardiol 1986; $57: 150-155$

Waldecker B, Coromilas J, Saltman A, et al. Overdrive stimulation of functional reentrant circuits causing ventricular tachycardia in the infarcted canine heart. Resetting and entrainment. Circulation 1993;87:(in press)

Waldo A, MacLean W, Karp R, et al. Entrainment and interruption of atrial flutter with atrial pacing: studies in man following open-heart surgery. Circulation 1977;56:737-748

Waldo A, Plumb V, Arciniegas J, et al. Transient entrainment and interruption of the atrioventricular bypass pathway type of paroxysmal atrial tachycardia. Circulation 1983;67:73-83

Waldo A, Henthorn R. Use of transient entrainment during ventricular tachycardia to localize a critical area in the reentry circuit for ablation. PACE 1989;12:231-244

Wellens H, Schuilenburg R, Durrer D. Electrical stimulation of the heart in patients with ventricular tachycardia. Circulation 1972;46:216-226 
Wellens H. Value and limitations of programmed electrical stimulation of the heart in the study and treatment of tachycardias. Circulation 1978;57:845-853

Wit A, Allessie M, Bonke F, et al. Electrophysiologic mapping to determine the mechanism of experimental ventricular tachycardia initiated by premalure impulses. Experimental approach and initial results demonstrating reentrant excitation. Am J Cardiol 1982;49:166-185

Zetelaki Z, Boersma L, Kirchhof C, et al. Mapping of polymorphic ventricular tachycardia in the isolated left ventricle of the rabbit. Submitted 


\section{SUMMARY}

In the chronic phase of myocardial infarction in patients, spontaneous ventricular tachycardia is frequently observed. Experimental evidence has been raised that these ventricular tachycardias are based on reentrant activation in thin sheets of surviving muscle overlying the infarct. The anatomic structure of the thin layers of myocardium seems to play an important role in the course of ventricular reentry. So far, it is not well known which types of reentrant excitation can occur in the chronic phase of myocardial infarction in patients. Also, the mechanisms by which programmed electrical stimulation and antiarrhythmic drugs abolish ventricular tachycardia or sometimes aggravate ventricular tachycardia are not well understood. This thesis describes several studies that were performed to determine the characteristics of different types of ventricular reentry that can be induced in thin layers of myocardium. For this purpose we tested the effects of programmed electrical stimulation and several antiarrhythmic drugs on conduction and on ventricular tachycardia in an experimental model.

The histologic and electrophysiologic characteristics of the experimental model used in this thesis are characterized in chapter 2 . A cryoprocedure was developed to destroy the complete right ventricle and the inner layers of the left ventricle in an isolated rabbit heart, resulting in a langendorff-perfused thin layer of ventricular muscle at the epicardial side of the heart. The heart could be electrically stimulated from the outside and the ventricular activation pattern was recorded with 248 electrodes at regular distances of $2.25 \mathrm{~mm}$. The cryoprocedure did not change the refractory periods nor their spatial dispersion in the surviving muscle sheet. During slow electrical stimulation the spread of activation in the sheet was uniform but had an ellipsoid shape. In the thin epicardial layer the muscle fibers were arranged parallel to each other. Comparison of the histologic and electrophysiologic data revealed that conduction along the epicardial fiber orientation was about three times faster than conduction transverse to the fiber direction (chapter 5). This anisotropy for conduction was even maintained when the conduction velocity in both directions was depressed during high concentrations of potassium.

During rapid electrical stimulation, arcs of local conduction block developed, leading to the induction of reentrant ventricular tachycardia (chapter 3). About $60 \%$ of these tachycardias terminated spontaneously within less than one minute, usually because the reentrant circuit slowly shifted to the boundary of the heart which annihilated the circulating wave front. Rarely, a new figure-of-eight tachycardia was formed that terminated by conduction block of the circulating waves in the central common pathway. During sustained ventricular tachycardia, micro-electrode recordings showed that when the circulating wave tried to make a sharp turn around the ends of the line of block, a local delay in activation occurred. As a result, the action potentials at the pivoting points were prolonged leading to an excitable gap in all the other parts of the circuit. In a number of hearts, a fixed anatomic obstacle was created by cryoablation, resulting in a ring of viable myocardium (chapter 4). In some segments of the ring conduction was fast because the impulse propagated parallel to the fiber orientation, whereas in other segments the impulse propagated transverse to the fiber orientation with a much slower conduction velocity. Electrical stimulation induced two wavefronts that propagated in opposite directions around the ring until they collided with each other. During early premature stimuli, one of the wavefronts was blocked, enabling the other wavefront to continue unopposed to initiate reentry around the obstacle. This unidirectional 
conduction block preferentially occurred during longitudinal impulse propagation. Spatial dispersion in refractoriness did not seem to be of influence. During all tachycardias the revolution time of the circulating wave around the obstacle exceeded the refractory period, resulting in a large excitable gap at all sites of the circuit.

The characteristics of reentry around a functional and an anatomic obstacle were studied by applying single premature stimuli (chapter 7). During anatomic reentry a large excitable gap was present at every site in the reentrant circuit and VT could always be reset by a premature stimulus. Three distinct local reset curves were found to identify the relative position of pacing and recording sites in the circuit. Functionally determined reentry was usually not reset by a premature stimulus, despite the presence of a small excitable gap in the circuit. In some cases, this was caused by the fact that the premature wavefront simply failed to enter the reentrant circuit. In the other cases, failure of reset was due to a slowing of conduction of the premature wave inside the circuit, indicating that the gap consisted of partially excitable tissue. The effects of entrainment with a train of closely coupled stimuli on anatomic reentry are described in chapter 8. At longer pacing intervals the tachycardia was transiently entrained and resumed its regular pattern after cessation of pacing. At very short pacing intervals, ventricular tachycardia was either terminated, changed in morphology or accelerated. Termination of the tachycardia could be due to complete conduction block of the paced circulating wave or to collision of the paced circulating wave with an antidromic echowave. A change in morphology occurred when the direction of propagation of VT was reversed. Acceleration of VT was due to introduction of a second circulating wave inside the same circuit, a phenomenon which was called double-wave reentry. All the interruptions of the tachycardia were preceded by conduction block, which preferentially occurred in the area of slow transverse impulse propagation. Prior to the occurrence of conduction block, local oscillations in interval at the site of block resulted in a short last interval that prohibited propagation of the paced circulating wave.

Chapters 6, 9, and 10 describe the effects of various paharmacological interventions on ventricular tachycardia around an anatomical obstacle. Chapter 6 discusses the effects of gradually raising the extracellular concentration of $\mathrm{K}^{+}$. High $\mathrm{K}^{+}$slowed the longitudinal conduction velocity more than conduction velocity in the transverse direction. The cycle length of tachycardia was gradually increased until at very high concentrations of $\mathrm{K}^{+}$the tachycardia was terminated. Conduction block of the circulating wave preferentially occurred in a segment where the impulse propagated parallel to the fiber orientation. Class Ic (Flecainide) and class III drugs (RP62719) were also able to terminate ventricular tachycardia around an anatomic obstacle (chapter 9). High concentrations of flecainide terminated tachycardia in every heart by a very strong depression of conduction. RP62719 could only terminate some tachycardias by increasing the refractory period at one site in the ring to such an extent that it exceeded the revolution time around the circuit, resulting in conduction block of the circulating wave. Both drugs usually terminated tachycardia by complete conduction block of the circulating wave. However, in some cases micro-reentry of the circulating wave iniated an antidromic echo-wave that collided with the circulating wave and thus terminated tachycardia. Chapter 9 shows how electrical uncoupling (heptanol), and class Ic (Org7797) and class III (d-Sotalol) drugs changed the excitable gap and the effects of entrainment during anatomic reentry. Heptanol and Org7797 were antiarrhythmic by slowing conduction and increasing the cycle length of ventricular tachycardia. However, since the drugs hardly increased refractoriness, the excitable gap was increased. In contrast to control, 
entrainment of ventricular tachycardia could now induce acceleration by double-wave reentry in all hearts. d-Sotalol increased the refractory period more than it increased the cycle length of ventricular tachycardia and thus decreased the excitable gap. As a result, in contrast to control, sustained acceleration of tachycardia by double-wave reentry was no longer possible.

\section{Conclusion}

In thin layers of anisotropic myocardium two different types of ventricular tachycardia based on reentrant excitation can be induced. The difference between these two types of tachycardia can be characterized by their response to single premature stimuli. The anisotropic conduction properties of the myocardium play an important role in initiation and termination of reentry around an anatomical obstacle. Interventions that diminish the excitability of the muscle cells preferentially depress longitudinal conduction, whereas uncoupling of the muscle cells preferentially depresses transverse conduction. By testing the effects of programmed electrical stimulation and antiarrhythmic drugs on reentry around anatomic obstacle, several new mechanisms for termination, changes in morphology, or acceleration of ventricular tachycardia have been identified. These results may contribute to our understanding of the characteristics of the reentrant pathway in the chronic phase of myocardial infarction in patients. They may also provide possible mechanisms for the antiarrhythmic and proarrhythmic actions of programmed electrical stimulation and antiarrhythmic drugs in patients. 


\section{SAMENVATTING}

Het hart is een holle spier die via ritmische contracties bloed door het lichaam pompt. De contractie van het hart komt tot stand door electrische prikkeling van de hartspiercellen die daarop samentrekken. De electrische impuls onstaat in de sinus-knoop in de rechterboezem van het hart en verplaatst zich via de boezems naar de hartkamers. In de verschillende fasen van een hartinfarct kan dit regelmatige activatie patroon van het hart verloren gaan. De ritmestoornissen in de chronische fase van het hartinfarct blijken te berusten op cirkelgeleiding van de electrische impuls in de hartkamers. Hierbij wordt de impuls als het ware gevangen in een circkelpad en is de hartfrequentie veel hoger dan normaal, een situatie die wordt aangeduid als kamertachycardie. Het cirkelpad wat aanleiding geeft tot deze kamertachycardie, blijkt te zijn gelokaliseerd in dunne lagen spierweefsel die het hartinfarct hebben overleefd. De anatomische structuur van deze dunne lagen spierweefsel lijkt een belangrijke rol te spelen in het beloop van kamertachycardieën. Ondanks vele studies is het nog niet geheel duidelijk welke vormen van cirkelgeleiding bij patiënten in de chronische fase van een hartinfarct kunnen optreden. Ook is nog niet goed bekend waarom behandeling van kamertachycardie met behulp van electrische stimulatie of geneesmiddelen al dan niet effectief is en soms zelfs leidt tot een verhoogde kans op ritmestoornissen. De studies in dit proefschrift werden uitgevoerd om te onderzoeken welke vormen van kamertachycardie kunnen optreden in dunne lagen gezond spierweefsel en wat de effecten van electrische stimulatie en antiaritmische geneesmiddelen op deze ritmestoornissen zijn.

Om de invloed van de anatomische structuur van het spierweefsel op het beloop van kamertachycardie te onderzoeken, is in ons laboratorium een dierexperimenteel model ontwikkeld wat wordt beschreven in hoofdstuk 2. Door middel van een vriestechniek worden de hele rechterkamer en de binnenzijde van de linkerkamer van geisoleerde konijneharten gedood, zodat er een dunne laag spierweefsel aan de buitenzijde van het hart overblijft. Het hart kan van buitenaf worden geprikkeld en de electrische activatie van de linkerkamerwand kan worden geregistreerd middels 248 electroden met een onderlinge afstand van $2.25 \mathrm{~mm}$. Tijdens langzame electrische stimulatie van de dunne spierlaag, verspreidt de electrische impuls zich volgens een regelmatig elliptisch patroon. In de dunne spierlaag zijn de hartcellen in lange evenwijdige banen gerangschikt. Vergelijking van het verloop van de spiercellen met het electrische activatie patroon liet zien dat electrische impulsgeleiding parallel aan de richting van de spiercellen bijna 3 maal sneller was dan geleiding dwars op deze spiervezelrichting, een verschijnsel dat anisotropie wordt genoemd (hoofdstuk 5). Ook wanneer de electrische impulsgeleiding in beide richtingen werd gedeprimeerd door een verhoging van de kaliumconcentratie in het hart, bleef deze anisotropie bestaan.

Wanneer de dunne anisotrope spierlaag met een hoge frequentie werd geprikkeld ontstond er lokaal geleidingsblok van de electrische impuls. In alle harten kon daardoor kamertachycardie worden opgewekt, gebaseerd op cirkelgeleiding rondom een lijn van lokaal functioneel geleidingsblok (hoofdstuk 3). In $60 \%$ van de harten stopte de kamertachycardie spontaan binnen een minuut, doordat de lijn van geleidingsblok langzaam verschoof naar de rand van het hart zodat de circulerende impuls niet meer verder kon geleiden. In de overige harten kon een landurige kamertachycardie worden opgewekt. Onderzoek van het cirkelpad met micro-electroden toonde aan dat de circulerende impuls werd vertraagd op het draaipunt van de lijn van geleidingsblok. Hierdoor waren de hartspiercellen in de overige delen van het cirkelpad al ruim hersteld voordat de 
volgende activatie door de circulende impuls plaatsvond, zodat er op de meeste plaatsen een klein "excitable gap" bestond.

In een aantal harten werd door bevriezing een blijvend anatomisch obstakel in de wand van de linkerkamer gemaakt, resulterend in een ring van spierweefsel (hoofdstuk 4). De geleidingssnelheid van de electrische impuls was niet gelijk in de verschillende delen van de ring. In sommige delen van de ring verliep de impulsgeleiding parallel aan de spiervezelrichting en was de geleidingssnelheid hoog, terwijl in andere delen van de ring trage geleiding optrad omdat de electrische impuls dwars op de spiervezelrichting voortgeleidde. Tijdens electrische stimulatie in de ring werden twee golffronten opgewekt die zich in tegenovergestelde richting rondom het obstakel voortbewogen totdat beide golffronten botsten en elkaar uitdoofden. In bijna alle harten bleek dat tijdens zeer vroege electrische prikkels één van beide golffronten blokkeerde zodat het andere golffront ongestoord kon voortgaan, waardoor kamertachycardie ontstond op basis van cirkelgeleiding rondom het anatomische obstakel. Geleidingsblok trad overwegend op in een deel van de ring waar de electrische impulsgeleiding parallel was aan de spiervezelrichting. Dit wijst erop dat tijdens vroege prikkels de longitudinale impulsgeleiding minder betrouwbaar is dan de transversale impulsgeleiding.

Het verschil in eigenschappen tussen cirkelgeleiding rondom een anatomisch obstakel en cirkelgeleiding rondom een lijn van functioneel geleidingsblok werd onderzocht door tijdens beide vormen van kamertachycardie vroege electrische prikkels toe te dienen (hoofdstuk 7). Tijdens cirkelgeleiding rondom een anatomisch obstakel was er een groot "excitable gap" omdat de geleidingstijd van de circulerende impuls rondom het obstakel veel langer was dan de tijd die de spiercellen nodig hadden om zich te herstellen van een voorgaande activatie (refractaire periode). Hierdoor konden vroege electrische prikkels het cirkelpad binnendringen en de kamertachycardie gedurende één cyclus versnellen. Ook tijdens cirkelgeleiding rondom een lijn van functioneel geleidingsblok kon een vroege electrische prikkel worden toegediend. Het "excitable gap" tijdens deze vorm van kamertachycardie was echter vrij klein en in de meerderheid van de harten kon de kamertachycardie niet kortdurend worden versneld. Tijdens cirkelgeleiding rondom een anatomisch obstakel werd het effect van een reeks vroege prikkels op het verloop van de kamertachycardie getest (hoofdstuk 8). Het bleek dat bij hoge stimulatie frequenties, geleidingsblok van één of meerdere gestimuleerde golffronten optrad. Geleidingsblok vond overwegend plaats tijdens transversale impulsgeleiding en werd gefaciliteerd door lokale oscillaties in het interval tussen twee opeenvolgende activaties. Geleidingsblok gaf meestal aanleiding tot het stoppen van de kamertachycardie, hetzij doordat de circulerende impuls compleet werd geblokkeerd ofwel doordat de circulerende impuls botste met een echo-golf uit de tegenovergestelde richting. In een aantal gevallen ontstond er een nieuwe kamertachycardie doordat de richting van de circulerende impuls volledig omdraaide. Soms werd de tachycardie bijna twee maal zo snel doordat er een tweede impuls in hetzelfde cirkelpad ging circuleren, een fenomeen dat "double-wave reentry werd genoemd.

De hoofdstukken 6, 9, en 10 beschrijven hoe verhoging van de kaliumconcentratie in het hart en toediening van heptanol en anti-aritmische geneesmiddelen uit de klasse Ic en klasse III groep de geleidingssnelheid tijdens cirkelgeleiding rondom een anatomisch obstakel beinvloeden. De resultaten in hoofdstuk 6 laten zien dat een stelselmatige verhoging van de kaliumconcentratie tijdens kamertachycardie, de longitudinale impulsgeleiding sterker vertraagde dan de transversale impulsgeleiding. Door de vertraging van de impulsgeleiding werd de kamertachycardie steeds trager totdat bij zeer hoge kalium- 
concentraties de tachycardie uiteindelijk werd gestopt door longitudinaal geleidingsblok van de circulerende impuls. Klasse Ic (flecainide) en klasse III (RP62719) antiaritmische geneesmiddelen bleken ook bij zeer hoge concentraties in staat om cirkelgeleiding rondom een anatomisch obstakel te stoppen (hoofdstuk 10). Flecainide kon in ieder hart de kamertachycardie stoppen door de impulsgeleiding zeer sterk te vertragen. RP'62719 kon slechts een beperkt aantal kamertachycardieën stoppen door de refractaire periode van de cellen zodanig te verlengen dat deze langer duurde dan de omlooptijd van de circulerende impuls die daardoor niet meer kon voortgeleiden. Bij beide geneesmiddelen kon terminatie optreden door compleet geleidingsblok van de circulerende impuls of door uitdoving van de circulerende impuls door een echo-golf uit de tegenovergestelde richting. In hoofdstuk 9 wordt beschreven hoe electrische ontkoppeling van de hartspiercellen (heptanol), en klasse Ic (Org7797) en klasse III (d-Sotalol) antiaritmische geneesmiddelen het effect van een reeks snelle prikkkels op cirkelgeleiding rondom een anatomisch obstakel veranderen. Heptanol en Org7797 hadden beiden een antiaritmisch effect doordat de geleidingssnelheid van de circulerende impuls afnam waardoor de omlooptijd van de kamertachycardia toenam. Doordat de refractaire periode echter nauwelijks werd verlengd werd ook het "excitable gap" groter met als nadelig gevolg dat nu een tweede circulerende impuls in het cirkelpad paste. In tegenstelling tot de controle omstandigheden kon een reeks snelle prikkels nu de kamertachycardie in alle harten versnellen door het opwekken van "double-wave reentry". d-Sotalol had juist een tegenovergesteld effect op de kamertachycardie doordat het de refractaire periode van de hartspiercellen meer verlengde dan de omlooptijd van de circulerende impuls. Hierdoor werd het "excitable gap" relatief kleiner zodat het niet langer mogelijk was om een langdurige versnelling van de kamertachycardie op te wekken.

\section{Beschouwing}

In een dunne laag anisotroop spierweefsel kunnen twee vormen van kamertachycardie op basis van cirkelgeleiding worden opgewekt. Deze vormen zijn te onderscheiden door de verschillende respons tijdens vroege electrische prikkels. De anisotrope geleidingseigenschappen in verschillende delen van een anatomische cirkelpad spelen een belangrijke rol bij het starten en stoppen van een kamertachycardie. Interventies die de prikkelbaarheid van de hartspiercellen verlagen werken sterker op de longitudinale impulsgeleiding terwijl transversale impulsgeleiding sterker wordt beinvloedt door interventies die de electrische koppeling tussen de cellen verminderen. Door de effecten van geprogrammeerde electrische stimulatie en anti-aritmische geneesmiddelen op cirkelgeleiding rondom een anatomisch obstakel te testen, werden een aantal nieuwe mechanismen ontdekt die verantwoordelijk kunnen zijn voor het stoppen, veranderen, of versnellen van een kamertachycardie. Deze gegevens kunnen wellicht meer inzicht verschaffen over de aard van het cirkelpad en de kamerritmestoornissen in de chronische fase van het hartinfarct bij patienten. Daarnaast bieden ze mogelijk enige aanknopingspunten om te begrijpen via welke mechanismen electrische stimulatie en geneesmiddelen het optreden van kamerritmestoornissen kunnen tegengaan of bevorderen. 


\section{NAWOORD}

Toen ik in 1987 als derde-jaars student Geneeskunde eens ging rondneuzen bij de vakgroep Electrofysiologie om "iets meer over hartritmestoornissen te leren" wist ik niet dat dit 7 jaar zou gaan duren. Destijds was het pingpongen in de gangen van het "oude" BioMedisch Centrum ook nog minstens zo spannend als mijn inwijding in de geheimen van het wetenschappelijk onderzoek. Het was in mijn ogen een vreemd groepje mensen wat daar "aan het onderzoeken was". Martin-Jan Schalij die iedere BMC'er benaderde alsof hij in de sociëteit van het corps in Utrecht stond, Charles Kirchhof die dagelijks tot middernacht bleef doorwerken, en Kees Augustijn die met de duim in de mond aan de computer zat. Ondanks (of misschien dankzij) deze vreemde atmosfeer werd de aantrekkingskracht om onderzoek te doen steeds groter. Ik was een jaar later op het juiste moment op de goede plek om de plaats van Martin-Jan Schalij in te nemen, waarvoor ik zelfs mijn studie geneeskunde moest onderbreken. In de daaropvolgende jaren heb ik met toenemend plezier bij de vakgroep Fysiologie gewerkt (slechts licht overschaduwd door het feit dat we bij de verhuizing naar de Universiteitssingel de kippen op de binnenplaats moesten achterlaten). Tijdens mijn onderzoek heb ik met veel mensen samengewerkt en uiteraard is het gepast om de bijdrage van sommigen nader toe te lichten.

Prof. Allessie, beste Maurits, hoewel onze wederzijdse verwachtingen over het doen van onderzoek niet altijd helemaal overeen kwamen, is de verstandhouding in de loop der jaren steeds beter geworden. Je creativiteit, snelle denkwerk en kritische benadering van het onderzoek vormden een bron van zowel inspiratie als frustratie (met name bij het schrijven van de zoveelste versie van een manuscript!). Ik kijk met plezier op onze samenwerking terug.

Dr. Brugada, dear Jep, we had a lot of fun during the three years that we worked together and that has been of great importance to complete this doctoral thesis. Your energy to do research is incredible and set an example for me. You're not bad for a guy with only three neurons. Hasta pronto en Banyoles!

De leden van de beoordelingscommissie Prof.H. Wellens, Prof.H.Struyker-Boudier, Prof. Th. Arts, Prof. M. Janse, en Prof. A. Wit, wil ik bedanken voor hun inzet en geduld om dit lijvige proefschrift te beoordelen en voor hun aanwezigheid tijdens de promotie. Paranymfen worden terecht met zorg gekozen. Des te opvallender is het dat het twee "boezem-boys" zijn! Charles Kirchof, ik ben blij dat je mijn paranymf wilt zijn, ondanks het feit dat je mij destijds het biercommisariaat in de maag hebt gesplitst. Je geduld om als "ombudsman" op te treden als het weer eens niet meezat was oneindig en ik heb bewondering voor je toewijding voor elke taak die je jezelf oplegt. Maurits Wijffels, ik vind het leuk dat jij als hockey-maatje van mij uiteindelijk ook bij de Electrofysiologie groep terecht bent gekomen. Ik weet zeker dat je onderzoek naar "boezem-flepperen" zeer succesvol zal zijn hoewel het je nog wel een paar zakken waspoeder, wasknijpers, en veel overredingskracht van de Milieucommissie gaat kosten om de komende jaren in je geitenlabje te overleven.

Martin-Jan Schalij, als uitvinder van het vrieshart-model ben je mede verantwoor-delijk voor het feit dat ik dit promotie-onderzoek heb kunnen doen. In mijn tijd als "tyrodeknecht" heb ik veel plezier beleefd aan de manier waarop je op Schalijaanse wijze de mensen in de kantine van het B.M.C. keer op keer weer wist te shoqueren. Ik hoop dat we in de toekomst weer eens zullen samenwerken.

In de latere fasen van mijn onderzoeksperiode kwamen Karen Konings, Zoltan Zetelaki, 
Rick Dormans, Kai Haberl, en Joost Frederiks bij Electrofysiologie werken. Hen allen wil ik bedanken voor de samenwerking en de goede sfeer die er op het lab heerste. Beste Karen, ik hoop dat je ondanks je besonjes als huisbazin, spoedig je onderzoek kunt afronden. Dear Zoltan, thank you for the cookies and the updates of the mapping system. Beste Rick, gezien de prestaties van Feyenoord in de Champions League is het maar goed dat Ajax dit jaar kampioen wordt! Dear Kai, thank's for allowing me to play "Wolffenstein" on your computer; I hope to visit you sometime below the "weisswurstequator". Beste Joost, jij bent de volgende tyrode-knecht die als waardige "ventrikel-boy" blijft hangen bij Electrofysiologie, daarvan ben ik overtuigd. Bedankt voor je hulp en gezelschap op het lab en tijdens de voorrondes van het Nederlands elftal en succes in je onderzoek.

There are always guests around our department: Michael Reiter, Javir Chorro, thank you for your scientific support and company (I still owe you both a diner!).

Jan Hollen en Frits Schmitz, het feit dat het werken met het mappingsysteem voor jullie een doel en voor mij slechts een middel was kwam soms wel eens slecht uit. Niettemin was jullie technische (en sociale) assistentie onmisbaar om überhaupt een proef te kunnen doen! We hebben zelfs om de flauwste grappen veel met elkaar gelachen. Doe het kalmpjes aan en eet niet te veel drop als ik weg ben!

Karen van Brussel, Claire Bollen, Karlijn Dickison, Jos Heemskerk, en Emmy van Roosmalen, bedankt voor de secretariële ondersteuning en het aanvullen van mijn voorraad plaksel. Beiden waren van wezenlijk belang om dit proefschrift te voltooien.

De mensen van de Centrale Proefdier Voorzieningen, met name Peter Kelderman, Brigit Kusters, en Frans Weekers, wil ik bedanken voor hun inzet om onze proeven mogelijk te maken.

Het schoonmaken van alle glaswerk en aanvullen van het gedestilleerd water was een ondankbare taak die ik graag en vaak aan de mensen van de spoelkeuken heb overgelaten, daarvoor mijn dank.

Op deze plaats is het ook gepast om mijn persoonlijke sponsors en collegae promovendi Marc Bemelmans en Maya Huyberts (oud-electrofysiologie, jawel) te bedanken voor het lenen van een van hun computers tijdens het schrijven van mijn proefschrift. Hij was niet snel maar deed het wel!

Het laatste maar niet minste woord van dank is gericht aan mijn ouders. Het is heel moeilijk om in een paar zinnen even te zeggen wat jullie voor mij betekenen. Anne en Wim, jullie hebben mij tijdens iedere nieuwe opleiding steeds weer bijgestaan om deze tot een goed einde te brengen. Jullie staan altijd klaar om mij te steunen als mijn stemming of motivatie "low" is. Het is geweldig om jullie als klankbord te hebben. Er zijn uiteraard mensen die ik vergeten ben: aan jullie, bedankt voor de samenwerking! 


\section{LIST OF PUBLICATIONS \\ (as first author)}

\section{Papers:}

1. Boersma L, Brugada J, Allessie M. Effects of $\mathrm{K}^{+}$and heptanol on conduction in anisotropic rabbit ventricular myocardium. New Trends Arrhyth 1991;7:91-96

2. Boersma L, Brugada J, Schalij MJ, Kirchhof C, Allessie MA. The effects of $\mathrm{K}^{+}$on anisotropic conduction in sheets of perfused rabbit ventricular myocardium. J Cardiovasc Electrophysiol 1991;2:492-502

3. Boersma L, Brugada J, Kirchhof C, Allessie M. Entrainment of ventricular tachycardia in anisotropic rings of rabbit myocardium: Mechanisms of termination, changes in morphology and acceleration. Circulation 1993;88:1852-1865

4. Boersma L, Brugada J, Kirchhof C, Allessie M. Mapping of reset of anatomic and functional reentry in anisotropic ventricular myocardium. Circulation 1994;89: (in press)

5. Boersma L, Brugada J, Abdollah H, Kirchhof C, Allessie M. Effects of heptanol, class Ic, and class III drugs on ventricular tachycardia. Importance of the excitable gap for the inducibility of double-wave reentry. Submitted

\section{Chapters:}

1. Boersma L, Brugada J, Kirchhof C, Allessie M. The effects of potassium on anisotropic conduction. In: Cardiac electrophysiology, circulation and transport. Eds: Sideman S, Beyar R, Kléber A. Kluwer Academic Publishers 1990, pp. 101-108

2. Boersma L, Brugada J, Allessie M. Pharmacological modulation of reentrant excitation in experimental models. In: Proceedings of the 1993 Munster Workshop. Antiarrhythmic drugs. Mechanisms of antiarrhythmic and proarrhythmic actions. Eds: Breithardt G, Camm J, Borggrefe M, Rosen M. Springer Verlag 1994, (in press)

\section{Abstracts:}

1. Boersma L, Schalij MJ, Musch E, Allessie M. The effects of potassium on conduction in anisotropic myocardium. Eur J Physiol (Pflügers Arch) 1989;14:S196

2. Boersma L, Brugada J, Kirchhof C, Allessie M. Resetting and entrainment of ventricular tachycardia in a ring of uniform anisotropic rabbit ventricular myocardium. Eur J Physiol (Pflügers Arch) 1990;416:S4

3. Boersma L, Brugada J, Kirchhof C, Allessie M. The effects of heptanol, high $\mathrm{K}^{+}$and TTX on reentrant ventricular tachycardia in anisotropic myocardium. Eur J Physiol (Pflügers Arch) 1991;418:R144

4. Boersma L, Brugada J, Kirchhof C, Allessie M. Drug-induced acceleration of reentrant ventricular tachycardia by double wave reentry: importance of the wavelength. Eur Heart J 1991;12:146

5. Boersma L, Brugada J, Kirchhof C, Allessie M. Differentiation of functional and anatomical reentrant tachycardia in anisotropic ventricular myocardium by the resetting 
response. Circulation 1991;86:II-667

6. Boersma L, Brugada J, Kirchhof C, Allessie M. The response to single extrastimuli characterizes functional or anatomical reentrant tachycardia in anisotropic ventricular myocardium. Eur J Physiol (Pflügers Arch) 1992;420:R90

7. Boersma L, Brugada J, Abdollah H, Kirchhof C, Allessie M. Heptanol and Org7797 enhance the susceptibility to acceleration of reentrant ventricular tachycardia by double-wave reentry during entrainment. Eur J Physiol (Pflügers Archiv) 1992;420:R90

8. Boersma L, Brugada J, Kirchhof C, Allessie MA. Echo-Wave termination during entrainment of reentrant ventricular tachycardia. PACE 1992;15:522

9. Boersma L, Brugada J, Kirchhof $C_{\text {, }}$ Allessie M. Enhanced interval oscillations precede termination of reentrant ventricular tachycardia by entrainment. Eur Heart J 1992;13:222

10. Boersma L, Brugada J, Kirchhof C, Allessie M. Echo-Wave termination by entrainment of reentrant ventricular tachycardia. Eur Heart J 1992;13:135

11. Boersma L, Brugada J, Kirchhof C, Allessie M. Mechanisms of termination of reentrant ventricular tachycardia by entrainment. Netherlands J Cardiol 1992;5:298

12. Boersma L, Brugada J, Kirchhof C, Allessie M. Termination of reentrant ventricular tachycardia by entrainment is preceded by enhanced interval oscillations at the site of block. Circulation 1992;86:531

13. Boersma L, Zetelaki Z, Brugada J, Frederiks J, Wijffels M, Kirchhof C, Allessie M. Non-sustained ventricular tachycardia is terminated spontaneously by shifting of the reentrant circuit to the boundary of the heart. Eur J Physiol (Pflügers Arch) 1993;424:R2

14. Boersma L, Zetelaki Z, Frederiks J, Wijffels M, Brugada J, Kirchhof C, Allessie M. Two mechanisms for spontaneous termination of polymorphic VT in isolated rabbit hearts. Circulation 1993;88:I-117

15. Boersma L, Brugada J, Reiter M, Abdollah $H$, Kirchhof C, Allessie $M$. The effects of antiarrhythmic drugs on the excitable gap determine the susceptibility to acceleration of ventricular tachycardia by double-wave reentry. Circulation 1993;88:I-446 


\section{CURRICULUM VITAE}

Boersma, Lucas Victor August

1965:

2 februari geboren te Heerlen

1977-1982:

HAVO, Stella Maris te Meerssen

1982-1984:

VWO, Stella Maris te Meerssen

1984-1988:

Doctoraal Examen Geneeskunde,

Rijksuniversiteit Limburg

1986-1987:

Student-assistent Klinische Psychiatrie,

Rijksuniversiteit Limburg

1987-1988:

Student-assistent Electrofysiologie, Rijksuniversiteit Limburg

1988-1993:

A.I.O. Vakgroep Fysiologie, Rijksuniversiteit Limburg

1990-heden:

Tweede fase Geneeskunde, Rijksuniversiteil Limburg

\section{Waarderingen}

1992:

1e prijs Posterpresentatie Najaarssymposium Nederlandse Vereniging voor Cardiologie 
Mapping of reentrant ventricular tachycardia in the rabbit heart

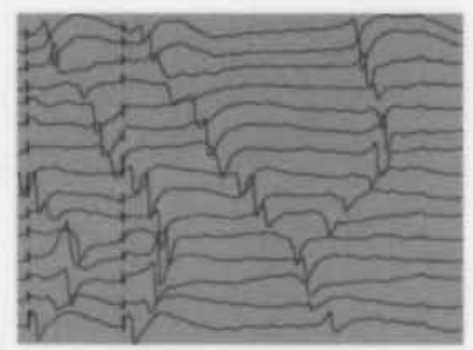

